\title{
An approach to categorification of some small quantum groups
}

\author{
Mikhail Khovanov, You Qi
}

May 20, 2013

\section{Contents}

1 Introduction $\quad 2$

2 Homological algebra of $p$-derivations $\quad 7$

2.1 The base category . . . . . . . . . . . . . . . . . . . . . . 7

$2.2 p$-DG algebras and $p$-DG modules . . . . . . . . . . . . . . . . . . . . . . . . . . . . . . .

2.3 Special cases ............................... 30

3 The $p$-DG nilHecke algebra 34

$3.1 p$-derivations on the nilHecke algebra . . . . . . . . . . . . . . 34

3.2 Specializations of $a \ldots \ldots \ldots \ldots$

3.3 Grothendieck ring as $u_{\mathbb{O}_{p}}^{+}\left(\mathfrak{s l}_{2}\right) \ldots \ldots \ldots$. . . . . . . . . . . . 60

4 The $p$-DG KLR algebra 68

4.1 -derivations on the KLR algebra . . . . . . . . . . . . . . . . . . . . . . .

4.2 Quantum Serre relations . . . . . . . . . . . . . . . 78

4.3 Grothendieck groups for small weights . . . . . . . . . . . . . 95

\begin{abstract}
We categorify one half of the small quantum $\mathrm{sl}(2)$ at a prime root of unity. An extension of this construction to an arbitrary simply-laced case is proposed.
\end{abstract}




\section{Introduction}

The equation $d^{2}=0$, together with the related notion of a chain complex and its (co)homology, plays a fundamental role in modern mathematics and physics. Shortly after the discovery of simplicial homology, Mayer [33, 34] suggested to remove the minus signs in the definition of the differential in the chain complex of a simplicial complex while working in characteristic $p$. The Mayer differential satisfies $d^{p}=0$ and gives rise to Mayer homology groups. Spanier [44] showed that the Mayer homology of a topological space can be expressed via the usual homology groups. Spanier's result closed the topic for almost half a century. The subject was brought back to life by Kapranov [15], Sarkaria [42, 43], Dubois-Violette [6, 7, 8], Kerner [9] and others, also see [1, 4, 14, 16, 46] and the references therein. These papers study a characteristic zero analogue of Mayer's generalized complexes, called $N$-complexes, with the generalized differential satisfying $d^{N}=0$. Defining such differentials often requires the use of a primitive $N$ th root of unity $q$ to twist classical constructions of the homological algebra. The subject is sometimes referred to as $q$-homological algebra.

Complexes of vector spaces constitute a symmetric monoidal category. A complex of vector spaces can be thought of as a graded module over the exterior algebra on one generator. The existence of a natural tensor product on complexes can be explained by the Hopf algebra structure of the exterior algebra, when viewed as an algebra in the category of super-vector spaces. Algebraic structures in the super world can be thickened using Majid's bosonisation procedure [30] to produce corresponding structures in the category of vector spaces. Grading (ubiquitous in homological algebra) can be interpreted in the language of comodules: a comodule over the group ring $\mathbb{k}[G]$ is the same as a $G$-graded $\mathbb{k}$-vector space.

Furthermore, as originally observed by Pareigis [38, the category of complexes is equivalent to the category of comodules over a suitable Hopf algebra, equipped with a cotriangular structure. This approach explains the symmetric monoidal tensor product of complexes via the Hopf algebra framework. Bichon [3] characterized $N$-complexes as comodules over a suitable Hopf algebra (the Borel subalgebra of the small quantum $\mathfrak{s l}_{2}$ at an $N$-th root of unity), generalizing Pareigis' work.

Given a Frobenius algebra $H$ over a field $\mathbb{k}$, its stable category $H-\underline{\bmod }$ is triangulated [13] (a morphism of $H$-modules is zero in the stable category if it factors through a projective module). Any finite-dimensional Hopf algebra is a Frobenius algebra, and its stable category is triangulated monoidal. Several years ago one of us suggested [18] to study module-categories over $H-\underline{\bmod }$ for suitable finite-dimensional Hopf algebras $H$. This was motivated by the observation that the Grothendieck ring of the stable category of finite-dimensional graded modules over the Hopf algebra $H=\mathbb{k}[x] /\left(x^{p}\right)$ with $\Delta(x)=x \otimes 1+1 \otimes x, \operatorname{deg}(x)=1$ for a field $\mathbb{k}$ of characteristic $p$ is naturally isomorphic to the ring of integers $\mathcal{O}_{p}$ in the cyclotomic field $\mathbb{Q}\left[\zeta_{p}\right]$ for the $p$-th primitive 
root of unity $\zeta_{p}=e^{\frac{2 \pi i}{p}}$ :

$$
K_{0}(H-\underline{\bmod }) \cong \mathcal{O}_{p} \cong \mathbb{Z}[q] /\left(1+q+\cdots+q^{p-1}\right),
$$

where $q$ is a formal variable (the ring of integers $\mathcal{O}_{N}$ in $\mathbb{Q}\left[\zeta_{N}\right]$ is generated by $\zeta_{N}=$ $e^{\frac{2 \pi i}{N}}$ over $\left.\mathbb{Z}\right)$. Witten-Reshetikhin-Turaev invariants of 3 -manifolds take values in the ring of cyclotomic integers $\mathcal{O}_{N}$ (in favorable cases; in general one needs to extend the ring to $\left.\mathcal{O}_{N}\left[\frac{1}{N}\right]\right)$. Categorified WRT invariants, if exist, might take values in a tensor triangulated category with the Grothendieck ring $\mathcal{O}_{N}$ or, even better, $\mathcal{O}_{N}\left[\frac{1}{N}\right]$.

We do not know any instances of monoidal triangulated categories whose Grothendieck ring contains $\mathbb{Z}\left[\frac{1}{N}\right]$ as a subring (it is an exciting problem to find such examples), and so should restrict to a simpler problem of categorifying $\mathcal{O}_{N}$. The ring $\mathcal{O}_{N}$ is isomorphic to $\mathbb{Z}[q] /\left(\Psi_{N}(q)\right)$, where $q$ is a formal variable and $\Psi_{N}$ the $N$-th cyclotomic polynomial. It seems that examples of monoidal triangulated categories with Grothendieck ring isomorphic to $\mathcal{O}_{N}$ are only known for $N$ a prime power $p^{r}$ or $2 p^{r}$. The cyclotomic polynomials for these $N$ are

$$
\Psi_{p^{r}}(q)=1+q^{p^{r-1}}+q^{2 p^{r-1}}+\cdots+q^{(p-1) p^{r-1}}
$$

and, for odd $p$,

$$
\Psi_{2 p^{r}}(q)=\Psi_{p^{r}}(-q)=1+(-q)^{p^{r-1}}+(-q)^{2 p^{r-1}}+\cdots+(-q)^{(p-1) p^{r-1}} .
$$

The fields $\mathbb{Q}\left[\zeta_{p^{r}}\right]$ and $\mathbb{Q}\left[\zeta_{2 p^{r}}\right]$ coincide, for odd $p$, and thus share the same ring of integers. The above example of categorification of $\mathcal{O}_{p}$ via the Hopf algebra $H$ can be easily modified, by changing the grading of generator $x$ from 1 to $p^{r-1}$, to a categorification of $\mathcal{O}_{p^{r}}$.

It would be thrilling to lift this example to characteristic 0 and to get rid of the $p^{r}$ restriction. There is a problem with doing it via $N$-complexes - for $N>2$ the base category of $N$-complexes modulo chain homotopies is neither symmetric nor braided, and its Grothendieck ring is isomorphic to $\mathbb{Z}[q] /\left(1+q+\cdots+q^{N-1}\right)$ rather than $\mathbb{Z}[q] /\left(\Psi_{N}(q)\right)$. We do not know how to circumvent these difficulties and construct a triangulated monoidal category with Grothendieck ring $\mathbb{Z}[q] /\left(\Psi_{N}(q)\right)$. For this reason we work in characteristic $p$, with Mayer's $p$-complexes, and so restrict our categorification attempts to $p$-th and $2 p$-th roots of unity.

To do this $p$-homological algebra, we need a supply of module-categories over the base symmetric triangulated category $H-\underline{\bmod }$. Given a graded $\mathbb{k}$-algebra $A$ with a degree one derivation $\partial$ (so that $\partial(a b)=\partial(a) b+a \partial(b))$ subject to the condition $\partial^{p}=0$, one can form the category $(A, \partial)-\bmod$ of $p$-DG modules over $A$, by analogy with the category of DG-modules over a DG-algebra, then pass to the homotopy category and, finally, localize by quasi-isomorphisms to get the analogue $\mathcal{D}(A, \partial)$ of the derived category. To have an interesting Grothendieck group, one can restrict to the subcategory $\mathcal{D}^{c}(A, \partial)$ of compact modules. For several reasons (mostly compatibility with prior 
work on categorification of quantum groups at generic $q$ ) we make our derivations have degree two. This results in the Grothendieck group of $\mathcal{D}^{c}(A, \partial)$ being a module over the ground ring

$$
\mathbb{O}_{p}=\mathbb{Z}[q] /\left(\Psi_{p}\left(q^{2}\right)\right),
$$

the Grothendieck ring of the stable category of finitely-dimensional $H$-modules. We denote the Grothendieck group by $K_{0}(A, \partial)$ or simply by $K_{0}(A)$. Derivation being of degree two translates into having $q^{2}$ in the formula. This polynomial is reducible, $\Psi_{p}\left(q^{2}\right)=\Psi_{p}(q) \Psi_{2 p}(q)$, so that $\mathbb{O}_{p}$ is not an integral domain, although it is a subring of the product $\mathcal{O}_{p} \times \mathcal{O}_{2 p}$ of two integral domains.

Categorification of tensor products of quantum group representations was obtained geometrically by Zheng [49], utilizing earlier ideas and constructions of Nakajima [36, 37] and Malkin [31], and by Webster [47, 48] in an algebro-combinatorial fashion, who also extended it to a categorification of Reshetikhin-Turaev link and tangle invariants [40]. One of the inputs in Webster's construction is a categorification of positive halves of quantum groups via diagrammatically described KLR rings [20, 21, 41]. To test whether $p$-complexes are relevant to categorification at prime roots of unity, we can try to integrate them with the categorification of quantum groups and their representations. A first step would be to look for nilpotent $\left(\partial^{p}=0\right)$ derivations with interesting properties on KLR rings $R(\nu)$.

We start with the $\mathfrak{s l}_{2}$ case, when the KLR rings $R(\nu)$ specialize to nilHecke algebras $\mathrm{NH}_{n}$. Categorification of multiplication and comultiplication of the positive half of quantum $\mathfrak{s l}_{2}$ at generic $q$ is achieved via induction and restriction functors for inclusions of algebras $\mathrm{NH}_{n} \otimes \mathrm{NH}_{m} \subset \mathrm{NH}_{n+m}$, see [20, 25]. The nilHecke algebra $\mathrm{NH}_{n}$ is isomorphic to the matrix algebra of size $n$ ! with coefficients in the ring of symmetric functions $\mathrm{Sym}_{n}$ in $n$ variables, and the $n$-th divided power $E^{(n)}=\frac{E^{n}}{[n] !}$ is categorified by the symbol of an indecomposable projective module $\mathcal{P}_{n}$ over $\mathrm{NH}_{n}$. The latter comes from realizing $\mathrm{NH}_{n}$ as the endomorphism ring of the space $\mathrm{Pol}_{n}$ of polynomials in $n$ variables over the subring $\operatorname{Sym}_{n}$ of symmetric polynomials, and $\mathcal{P}_{n}$ is identified with $\mathrm{Pol}_{n}$, up to a grading shift.

Working over a field $\mathbb{k}$ of characteristic $p$, we construct a family of derivations $\partial_{a}$, parametrized by $a \in \mathbb{F}_{p}$, i.e., by residues modulo $p$. These satisfy $\partial_{a}^{p}=0$ and have a local description, given on generators by

$$
\left.\partial_{a}(\boldsymbol{\phi})=\boldsymbol{\phi}^{2}, \quad \partial_{a}\left(\zeta^{\prime}\right)=a|-(a+1)\rangle+(a-1)\right\rangle
$$

The derivation $\partial_{a}$ on $\mathrm{NH}_{n}$ comes from a suitable derivation $\partial_{\alpha}$ on the polynomial space $\mathrm{Pol}_{n}$. Inclusions

$$
\mathrm{NH}_{n} \otimes \mathrm{NH}_{m} \subset \mathrm{NH}_{n+m}
$$

commute with $\partial_{a}$ and give induction and restriction functors between categories of $p$-DG modules over these algebras and corresponding derived categories. 
The positive half of the small quantum $\mathfrak{s l}_{2}$ has one generator $E$ and one defining relation $E^{p}=0$. In our categorification $E$ becomes the image in the Grothendieck group of an object $\mathcal{P}$, and the hom spaces $\operatorname{HOM}\left(\mathcal{P}^{\otimes n}, \mathcal{P}^{\otimes m}\right)=0$ for $n \neq m$, while $\operatorname{HOM}\left(\mathcal{P}^{\otimes n}, \mathcal{P}^{\otimes n}\right)=\operatorname{END}\left(\mathcal{P}^{\otimes n}\right)=\mathrm{NH}_{n}$, equipped with derivation $\partial_{a}$. We show that when $n=p$ and $a \neq 0$, the endomorphism algebra $\operatorname{END}\left(\mathcal{P}^{\otimes p}\right)=\mathrm{NH}_{p}$ is acyclic, as an $H$-module, and its derived category is trivial, implying that the object $\mathcal{P}^{\otimes p}$ is isomorphic to the zero object in the derived category. We view this result as a categorification of the defining relation $E^{p}=0$ in the small quantum group. When $a=0$, the $p$-DG algebra $\mathrm{NH}_{p}$ is not acyclic, preventing the derived category from being trivial and leading us to exclude this case by restricting to $a \in \mathbb{F}_{p}^{*}$.

A twisted version of the restriction functor for the inclusion (5) of $p$-DG algebras induces a homomorphism of Grothendieck groups

$$
K_{0}\left(\mathrm{NH}_{n+m}\right) \longrightarrow K_{0}\left(\mathrm{NH}_{n} \otimes \mathrm{NH}_{m}\right)
$$

(we write $K_{0}\left(\mathrm{NH}_{n+m}\right)$ instead of $K_{0}\left(\mathrm{NH}_{n+m}, \partial_{a}\right)$, etc.) For this map to categorify the comultiplication in a bialgebra requires an isomorphism

$$
K_{0}\left(\mathrm{NH}_{n} \otimes \mathrm{NH}_{m}\right) \cong K_{0}\left(\mathrm{NH}_{n}\right) \otimes_{\mathbb{O}_{p}} K_{0}\left(\mathrm{NH}_{m}\right),
$$

where $\mathbb{O}_{p}$ is the ground ring (the Grothendieck ring of the stable category of finitedimensional $H$-modules).

Only for $a= \pm 1$ are we able to establish this isomorphism as well as to show that the Grothendieck group of the $p$ - $\mathrm{DG}$ algebra $\mathrm{NH}_{n}$ (for $n<p$ ) equipped with derivation $\partial_{a}$ is a free rank one $\mathbb{O}_{p}$-module. This $\mathbb{O}_{p}$-module is generated by the symbol $\left[\mathcal{P}_{n}(\alpha)\right]$ of the indecomposable projective $\mathrm{NH}_{n}$-module carrying a derivation $\partial_{\alpha}$. The $p$-DGmodule $\mathcal{P}_{n}(\alpha)$ categorifies the $n$-th divided power $E^{(n)}=\frac{E^{n}}{[n] !}$ of the generator $E$. We do not know the structure of $K_{0}\left(\mathrm{NH}_{n}, \partial_{a}\right)$ for other values of $a$.

For $a= \pm 1$ induction and twisted restriction functors lead to a $q$-bialgebra (or twisted bialgebra) structure on the direct sum of Grothendieck groups $K_{0}\left(\mathrm{NH}_{n}, \partial_{a}\right)$. We identify this $q$-bialgebra with an integral form of the positive half of the small quantum $\mathfrak{s l}_{2}$ at a $2 p$-th root of unity (Theorem 3.35):

$$
K_{0}\left(\mathrm{NH}, \partial_{ \pm 1}\right) \cong u_{\mathbb{O}_{p}}^{+}\left(\mathfrak{s l}_{2}\right) .
$$

The integral form is a free $\mathbb{O}_{p}$-module with basis elements $E^{(n)}$ for $0 \leq n \leq p-1$ and the usual multiplication and comultiplication rules, see Section 3.3 ,

To an oriented graph $\Gamma$ there is assigned [20, 21, 41] a family of graded $\mathbb{k}$-algebras $R(\nu)$, over positive integral weights $\nu$. The direct sum of Grothendieck groups of finitelygenerated projective graded $R(\nu)$-modules, over all $\nu$, can be canonically identified [20, [21, 45] with an integral form of the positive half of the quantum group associated to $\Gamma$ :

$$
\mathrm{U}_{q, \mathbb{Z}}^{+}(\Gamma) \cong \underset{\nu \in \mathbb{N}^{I}}{\oplus} K_{0}(R(\nu)) .
$$


In particular, Serre relations hold on the categorical level, lifting to isomorphisms between certain direct sums of projective $R(\nu)$-modules.

Each vertex $i$ of $\Gamma$ generates nilHecke algebras $R(n i) \cong \mathrm{NH}_{n}$ as $R(\nu)$ for $\nu=n i$. Assigning $a_{i} \in \mathbb{F}_{p}^{*}$ to a vertex $i$ gives us derivations $\partial_{a_{i}}$ on $R(n i)$. These derivations extend in a multi-parameter way, written down in Section 4, to derivations $\partial$ on $R(\nu)$ for all weights $\nu$. We show in Section 4 that having Serre relations on the categorical level requires $a_{i}=a_{j}$ for $i, j$ in the same connected component of $\Gamma$ and $a_{i} \in\{1,-1\}$ for each $i$. Assuming $\Gamma$ is connected, this forces all $a_{i}$ to be equal 1 or -1 . We can restrict to $a_{i}=1$, for all $i$; the opposite case follows by applying an automorphism to rings $R(\nu)$. Furthermore, having Serre relations on the Grothendieck group level requires a unique choice for all other parameters as well, once one of the two cases ( 1 or -1$)$ is chosen for the $a_{i}$ 's. We work with the case $a_{i}=1, i \in I$, and obtain a canonical derivation $\partial_{1}$ on all $R(\nu)$, given in Definition 4.13 and extending derivation $\partial_{1}$ of $\mathrm{NH}_{n}=R(n i)$. Our results on categorification of the Serre relations are summarized in Theorem 4.14 . Conjecture 4.18 conveys our hopes that $p$-DG rings $R(\nu)$, over all weights $\nu$, categorify a positive half of the small quantum group associated with the graph $\Gamma$.

Other algebraic objects that appear in categorification also carry $p$-nilpotent derivations and have a potential to categorify corresponding quantum structures at prime roots of unity. For instance, in [10], it is shown that Lauda's two-category $\mathcal{U}$ admits a $p$-nilpotent derivation leading to a categorification of an integral form of the small dotted quantum group $\dot{u}_{\zeta_{2 p}}\left(\mathfrak{s l}_{2}\right)$. For another example, Webster's algebras [47], which categorify tensor products of simple modules over quantum Kac-Moody algebras, carry a multi-parameter family of nilpotent derivations; the $\mathfrak{s l}_{2}$ case is briefly discussed at the end of this paper. Thick calculus of [22] coupled with suitable derivations promises a categorification of the big quantum $\mathfrak{s l}_{2}$ at a prime root of unity [11].

In 1994 Louis Crane and Igor Frenkel conjectured [5] that there exists a categorification of the small quantum group $\mathfrak{s l}_{2}$ at roots of unity and that a categorical lift of $\mathrm{Ku}-$ perberg's 3-manifold invariant [24] should give a quantum invariant of four-manifolds. Igor Frenkel developed the ideas of categorification much further, in unpublished notes on structural constraints in categorified quantum $\mathfrak{s l}_{2}$, in many of his follow-up joint papers on categorification and geometrization of representation theory, and while advising several graduate students who went on to further grow the categorification program, including the senior author of the present paper. The junior author is a second-generation student of Igor Frenkel. We are delighted to dedicate this work to Igor Frenkel, on the occasion of his sixtieth birthday.

Acknowledgments. We are grateful to Ben Elias, Alexander Ellis, Aaron Lauda, Joshua Sussan and the anonymous referees for carefully reading early drafts of the paper and suggesting many corrections. Krzysztof Putyra wrote a latex package which allowed us to produce all diagrams in Sections 3 and 4 . The authors were partially supported by NSF grants DMS-1005750 and DMS-0739392 while working on the paper. 


\section{Homological algebra of $p$-derivations}

\subsection{The base category}

The Hopf algebra $H$ and its category of modules. We work over a field $\mathbb{k}$ of prime characteristic $p$, so that there is a natural inclusion of fields $\mathbb{F}_{p}=\mathbb{Z} /(p) \subset \mathbb{k}$. The algebra $H=\mathbb{k}[\partial] /\left(\partial^{p}\right)$ is a Hopf algebra, with comultiplication $\Delta(\partial)=1 \otimes \partial+$ $\partial \otimes 1$, antipode $S(\partial)=-\partial$, and counit $\epsilon\left(\partial^{i}\right)=\delta_{0, i}$. We make $H$ into a $\mathbb{Z}$-graded Hopf algebra via $\operatorname{deg}(\partial)=2$. Let $H-\bmod$ be the category of graded $H$-modules (morphisms are grading-preserving module maps) and $H$-fmod the subcategory of finite-dimensional graded $H$-modules. We also call objects of $H$-mod $p$-complexes and denote the vector space of (grading-preserving) homomorphisms between $p$-complexes $U$ and $W$ by $\operatorname{Hom}_{H}(U, W)$.

Any indecomposable object of $H$-mod is isomorphic, up to a grading shift, to $V_{i}:=H /\left(\partial^{i+1}\right)$ for some $0 \leq i \leq p-1$. Define the balanced indecomposable by $\widetilde{V}_{i}=V_{i}\{-i\}$. It has a copy of the ground field in degrees $-i,-i+2, \ldots, i$, and we denote the basis elements by $\tilde{v}_{k}$. so that $\widetilde{V}_{i}=\oplus_{k=0}^{i} \mathbb{k} \tilde{v}_{k}$, with $\partial\left(\tilde{v}_{k}\right)=\tilde{v}_{k+1}$ for $k<i$ and $\partial\left(\tilde{v}_{i}\right)=0$.

The categories $H$-mod and $H$-fmod have the Krull-Schmidt unique decomposition property. The cocommutative Hopf algebra structure of $H$ turns $H$-mod and $H$-fmod into symmetric monoidal categories, with $\partial$ acting on $U \otimes W$ via $\partial(u \otimes w)=\partial(u) \otimes$ $w+u \otimes \partial(w)$. The tensor product of indecomposable objects is given by

$$
\widetilde{V}_{i} \otimes \widetilde{V}_{j} \cong\left(\underset{m \in I}{\oplus} \widetilde{V}_{m}\right) \oplus \widetilde{V}_{p-1}^{[\max (0, i+j+2-p)]}
$$

where $I=\{|i-j|,|i-j|+2, \ldots, \min (i+j, 2 p-i-j-4)\}$ and $[n]=q^{n-1}+q^{n-3}+\cdots+$ $q^{1-n} \in \mathbb{N}\left[q, q^{-1}\right]$ for $n=\max (0, i+j+2-p)$ is the multiplicity, with grading shifts, of the free module $\widetilde{V}_{p-1}$ in the tensor product. Notice that $I$ is the empty set if $i=p-1$ or $j=p-1$, since the tensor product of a free module over a Hopf algebra with any module is free. We have

$$
V_{i} \otimes V_{p-1} \cong V_{p-1} \oplus V_{p-1}\{2\} \oplus \cdots \oplus V_{p-1}\{2 i\}
$$

The antipode $S$ gives us a contravariant functor on $H-\bmod$ taking $U$ to $U^{*}=$ $\oplus_{i \in \mathbb{Z}} \operatorname{Hom}_{\mathbb{k}}\left(U^{i}, \mathbb{k}\right)$ with $(\partial f)(u)=-f(\partial(u))$, where $U^{i}$ stands for the homogeneous degree $i$ part of $U$. This functor restricts to a contravariant involutive self-equivalence on $H$-fmod. Internal homs in $H$ - mod are given by

$$
\operatorname{HOM}_{\mathbb{k}}(U, W):=\oplus_{i, j \in \mathbb{Z}} \operatorname{Hom}_{\mathbb{k}}\left(U^{i}, W^{j}\right), \quad(\partial f)(u)=\partial(f(u))-f(\partial(u)) .
$$

Internal homs are graded whose homogeneous terms are given by

$$
\operatorname{HOM}_{\mathbb{k}}^{k}(U, W):=\oplus_{j-i=k} \operatorname{Hom}_{\mathbb{k}}\left(U^{i}, W^{j}\right)
$$


for any $k \in \mathbb{Z}$. In the smaller category $H-$ fmod the above direct sum coincides with $\oplus_{i \in Z} \operatorname{Hom}_{\mathbb{k}}(U\{i\}, W)$. We also define

$$
\operatorname{HOM}_{H}(U, W):=\bigoplus_{i \in \mathbb{Z}} \operatorname{Hom}_{H}(U\{i\}, W)
$$

Balanced modules are self-dual: $\widetilde{V}_{i} \cong \operatorname{HOM}_{\mathbb{k}}\left(\widetilde{V}_{i}, \mathbb{k}\right)$.

The Grothendieck ring $K_{0}(H-$ fmod) of the symmetric monoidal abelian category $H$ - fmod is naturally isomorphic to the ring of Laurent polynomials $\mathbb{Z}\left[q, q^{-1}\right]$, with $1=\left[V_{0}\right]$, grading shift corresponding to multiplication by $q:[U\{m\}]=q^{m}[U]$, and the symbol $[U]$ of any $p$-complex $U$ given by its graded dimension. In particular,

$$
\left[V_{i}\right]=1+q^{2}+\cdots+q^{2(i-1)}, \quad\left[\widetilde{V}_{i}\right]=[i]:=q^{i-1}+q^{i-3}+\cdots+q^{1-i} .
$$

Stable category and triangular structure. Any graded projective $H$-module is a free graded $H$-module, isomorphic to a direct sum of copies of $H$ with shifts. Taking the graded dual $\widetilde{V}_{p-1}^{*} \cong \widetilde{V}_{p-1}$, we see that projective modules are also injective and vice versa. Therefore the category $H-\bmod$ is Frobenius ([13]). Define the stable category $H-\underline{\bmod }$ to have the same objects as $H-\bmod$ and morphisms $\operatorname{Hom}_{H-\underline{\bmod }}(U, W)$ to be the quotient of the space of morphisms $\operatorname{Hom}(U, W)$ in $H$-mod by the subspace of morphisms that factor through a projective object. Define $H-\underline{\bmod }$ and $\mathrm{HOM}_{H-\mathrm{fmod}}(U, W)$ likewise.

A morphism $f: U \longrightarrow W$ factors through a projective object if and only if it factors through the canonical $H$-module map

$$
\lambda_{U}: U \longrightarrow H\{2-2 p\} \otimes U, \quad \lambda_{U}(u)=\partial^{p-1} \otimes u
$$

see [18, Lemma 1]. A map $g: H\{2-2 p\} \otimes U \longrightarrow W$ is determined by $h: U\{2-$ $2 p\} \longrightarrow W$, where $h(u):=g(1 \otimes u)$. Define $g_{i}(u):=g\left(\partial^{i} \otimes u\right)$. Then $h(u)=g_{0}(u)$ and $g_{i+1}(u)=\partial\left(g_{i}(u)\right)-g_{i}(\partial(u))$, implying 1

$$
g_{i}=\sum_{j=0}^{i}(-1)^{j}\left(\begin{array}{l}
i \\
j
\end{array}\right) \partial^{i-j} \circ g_{0} \circ \partial^{j} .
$$

In particular ,

$$
g_{p-1}=\sum_{j=0}^{p-1} \partial^{p-1-j} \circ h \circ \partial^{j}
$$

since

$$
(-1)^{j}\left(\begin{array}{c}
p-1 \\
j
\end{array}\right) \equiv 1(\bmod p)
$$

\footnotetext{
${ }^{1}$ Here we use "o" to denote the composition of maps. In what follows, to avoid potential confusion, we will use "." to denote the $H$-action on Hom-spaces when necessary.
} 
Therefore, $f: U \longrightarrow W$ factors through a projective $p$-complex if and only if there is $\mathbb{k}$-linear map $h: U \longrightarrow W$ of degree $2-2 p$ such that

$$
f=\sum_{j=0}^{p-1} \partial_{W}^{p-1-j} \circ h \circ \partial_{U}^{j} .
$$

Here "o" denotes composition of $\mathbb{k}$-linear maps. The following diagram illustrates an $f$ and $h$ of the formula. One should compare this with the usual notion of a null-homotopic map between complexes.

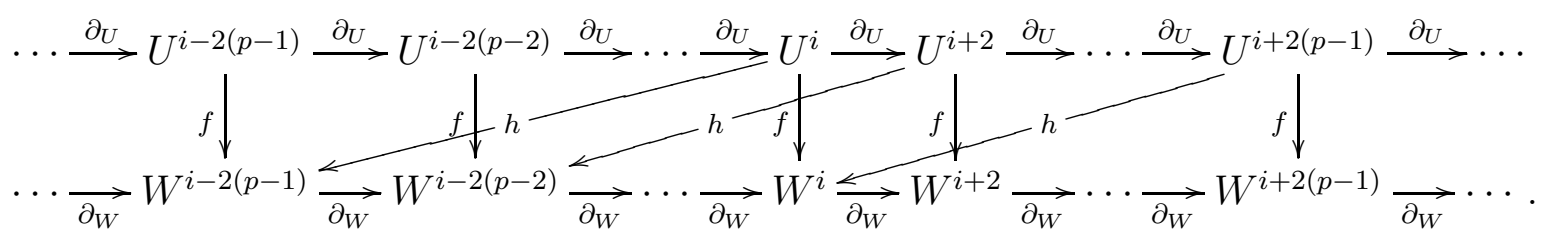

Definition 2.1. We call a $p$-complex $U$ contractible if it is isomorphic to the zero complex in $H-\underline{\bmod }$.

Equivalently, from the discussion above, $U$ is contractible if and only if the identity morphism $\operatorname{Id}_{U}: U \longrightarrow U$ factors through the canonical injection (9). We summarize the definition and the above equational characterization into the following proposition.

Proposition 2.2. The following conditions on a p-complex $U$ are equivalent:

i) $U$ is contractible.

ii) $U$ is a graded projective $H$-module.

iii) $U$ is a graded injective $H$-module.

iv) $U$ is isomorphic to a direct sum of graded shifts $V_{p-1}\{i\}$ of the free rank one module $V_{p-1}$.

$v)$ For any $x \in U$ with $\partial(x)=0$ there exists an $y \in U$ such that $\partial^{p-1}(y)=x$.

vi) There exists a linear endomorphism $h$ of $U$ of degree $2-2 p$ such that

$$
\operatorname{Id}_{U}=\sum_{j=0}^{p-1} \partial^{p-1-j} \circ h \circ \partial^{j}
$$

Proof. Obvious from the definition and discussion above. 
Once and for all, we fix an inclusion of balanced indecomposable modules

$$
\tilde{\iota}: \widetilde{V}_{0} \longrightarrow \widetilde{V}_{p-2} \otimes \widetilde{V}_{p-2}, \quad \tilde{\iota}\left(\tilde{v}_{0}\right):=\sum_{j=0}^{p-2}(-1)^{j} \tilde{v}_{j} \otimes \tilde{v}_{p-2-j} .
$$

The decomposition (8) shows that $\tilde{\iota}$ induces an isomorphism in the stable category $H-\underline{\bmod }$.

We define the shift functor [1] on $H-\underline{\bmod }$ and its full subcategory $H-\underline{\text { fmod }}$ as follows. For any $U \in H-\underline{\bmod }$, let

$$
U[1]:=(H \otimes U)\{2-2 p\} /\left(\operatorname{Im} \lambda_{U}\right)=\widetilde{V}_{p-2}\{-p\} \otimes U .
$$

Then [1] is invertible and its inverse functor is given by

$$
[-1]: U \mapsto \widetilde{V}_{p-2}\{p\} \otimes U=V_{p-2}\{2\} \otimes U .
$$

The map $\tilde{\iota}$ fixes functor isomorphisms $\operatorname{Id} \cong[-1][1]$ and $\{-2 p\} \cong[2]$.

The class of exact triangles in $H-\underline{\bmod }$ (resp. $H-\underline{\mathrm{fmod}}$ ) is constructed as follows. Let $f: U \longrightarrow V$ be a morphism in $H-\underline{\bmod }$ and let $f$ be a lift of $f$ in $H-\bmod$. Consider the push-out of $\lambda_{U}$ and $f$, which fits into the following commutative diagram:

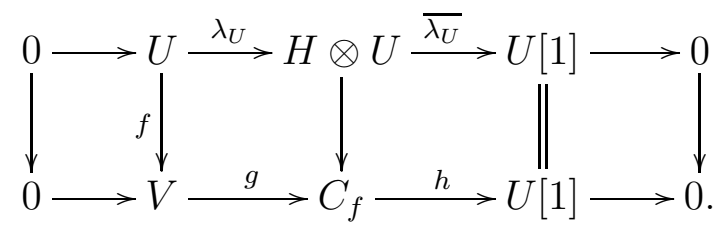

Then we declare the sextuple $U \stackrel{\stackrel{f}{\longrightarrow}}{\longrightarrow} V \stackrel{\underline{g}}{\longrightarrow} C_{f} \stackrel{\underline{h}}{\longrightarrow} U[1]$ to be a standard exact triangle, where $\underline{g}, \underline{h}$ denotes the image of $g$ and $h$ in $H$ - $\underline{\bmod }$. Moreover, any sextuple $U \stackrel{f}{\longrightarrow} V \stackrel{\underline{g}}{\longrightarrow} W \stackrel{\underline{h}}{\longrightarrow} U[1]$ that is isomorphic in $H-\underline{\bmod }$ to a standard exact triangle is called an exact triangle. The notation $\underline{f}, \overline{\lambda_{U}}$, etc. is taken from Happel [13].

Proposition 2.3. The category of p-complexes $H$ - $\underline{\bmod }$ (resp. $H$ - $\underline{\text { fmod) }}$ equipped with the shift functor [1] and the class of exact triangles as above is triangulated.

Proof. Omitted. This is a special case of Theorem 2.6 in [13, Section 1.2]

The shift $U[1]$ of an object $U$ can be computed more economically by embedding $U$ into a contractible complex and taking the quotient. For instance, the balanced indecomposable $\widetilde{V}_{i}$ is a submodule of the contractible module $\widetilde{V}_{p-1}\{i+1-p\}$, and $\widetilde{V}_{i}[1] \cong \widetilde{V}_{p-1}\{i+1-p\} / \widetilde{V}_{i} \cong \widetilde{V}_{p-2-i}\{-p\}$. The picture below illustrates this for $p=5$ 
and $i=2$. Each one-dimensional weight space of is denoted by a dot and the $\partial$ action by horizontal arrows. The degree zero spaces are labeled by black dots.

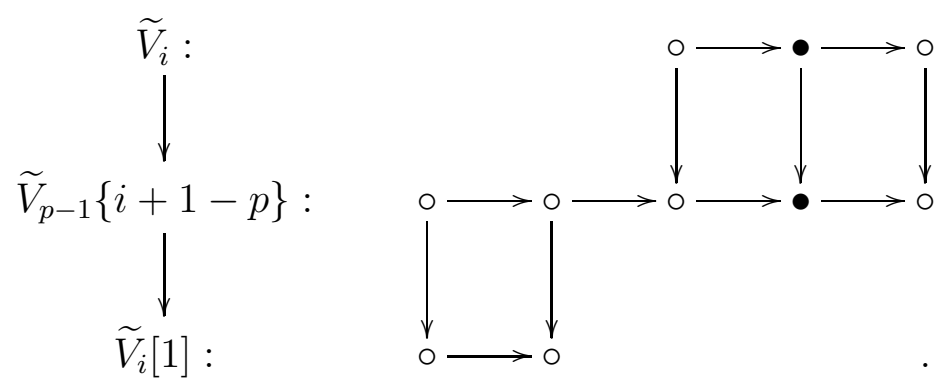

Reading these diagrams bottom-up with an appropriate grading shift is a convenient way to realize the functor $[-1]$ on balanced indecomposable $H$-modules.

We also record the following useful result on how short exact sequences of $H$-modules give rise to exact triangles in $H-\underline{\bmod }$. Suppose $0 \longrightarrow U \stackrel{f}{\longrightarrow} V \stackrel{g}{\longrightarrow} W \longrightarrow 0$ is a short exact sequence of $H$-modules. Consider the diagram

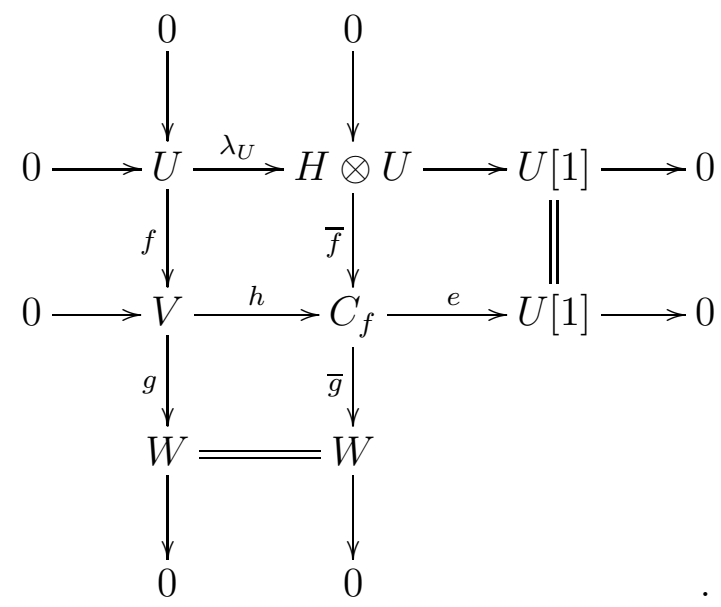

Here $C_{f}$ is the push-out of $f$ and $\lambda_{U}$, and the rest of the diagram is completed by the push-out property. We claim the $\bar{g}: C_{f} \longrightarrow W$ is an isomorphism in $H$ - mod. In fact, the submodule $H \otimes U$ is injective, and thus the middle vertical exact sequence splits and gives us $C_{f} \cong(H \otimes U) \oplus W$. The claim follows.

Lemma 2.4. In the above notation,

$$
U \stackrel{\stackrel{f}{\longrightarrow}}{\longrightarrow} V \stackrel{\underline{h}}{\longrightarrow} W \stackrel{\underline{e} \cdot \bar{g}^{-1}}{\longrightarrow} U[1]
$$

is an exact triangle. Conversely any exact triangle in $H-\underline{\bmod }$ is isomorphic to one of this form. 
Proof. By the remarks made before the lemma, this sextuple is isomorphic to the standard one

$$
U \stackrel{\stackrel{f}{\longrightarrow}}{\longrightarrow} V \stackrel{\underline{h}}{\longrightarrow} C_{f} \stackrel{\underline{e}}{\longrightarrow} U[1] .
$$

The result follows.

The Grothendieck ring of the stable category. Since the tensor product of any $p$-complex with a contractible $p$-complex is contractible, the tensor product bifunctor $\otimes: H-\bmod \times H-\bmod \longrightarrow H-\bmod$ descends to the stable categories $H-\underline{\bmod }$ and $H$ - $\underline{\text { mod }}$ and turns them into symmetric monoidal categories.

The tensor product of balanced indecomposables in the stable category is described by

$$
\tilde{V}_{i} \otimes \tilde{V}_{j} \cong \underset{m \in I}{\oplus} \widetilde{V}_{m}
$$

where $I=\{|i-j|,|i-j|+2, \ldots, \min (i+j, 2 p-i-j-4)\}$, see earlier. Notice that $U \otimes W \cong 0$ in $H-\underline{\bmod }$ if and only if either $U \cong 0$ or $W \cong 0$.

Lemma 2.5. The symmetric monoidal structure $\otimes: H-\underline{\bmod } \times H-\underline{\bmod } \longrightarrow H-\underline{\bmod }$ is compatible with the triangulated structure on $H-\underline{\bmod }$, in the sense that for any $U, W \in H-\underline{\bmod }$,

$$
(U \otimes W)[1] \cong U[1] \otimes W \cong U \otimes(W[1]) .
$$

Proof. Immediate, since $U[1]=\widetilde{V}_{p-2}\{-p\} \otimes U$ and $H$ is cocommutative.

Later we will see that the natural inclusion $H-\underline{\text { mod }} \subset H$ - $\underline{\bmod }$ induces an equivalence of $H$ - $\underline{\text { fmod }}$ with the subcategory $H-\underline{\bmod }^{c}$ consisting of compact objects.

Definition 2.6. We define the Grothendieck group $K_{0}(H-\underline{\text { fmod }})$ to be the abelian group generated by the symbols $[U]$ of isomorphism classes of objects $U \in H-\underline{\mathrm{fmod}}$, subject to the relations that $[V]=[U]+[W]$ whenever $U \longrightarrow V \longrightarrow W \longrightarrow U[1]$ is an exact triangle.

The exact triangle $U \longrightarrow 0 \longrightarrow U[1] \stackrel{-\operatorname{Id}_{U[1]}}{\longrightarrow} U[1]$ gives the relation $[U[1]]=-[U]$

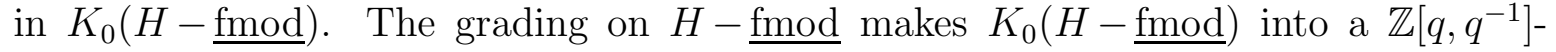
module by setting $q[U]:=[U\{1\}]$, while the exact symmetric monoidal structure on $H-\underline{\text { mod }}$ equips $K_{0}(H-\underline{\text { fmod }})$ with a commutative ring structure, $1:=\left[V_{0}\right]$ being the multiplicative unit.

Clearly, $K_{0}(H-\underline{\text { fmod }})$ is generated as a $\mathbb{Z}\left[q, q^{-1}\right]$-module by the symbol $\left[V_{0}\right]$, subject to the only relation that $\left(1+q^{2}+\cdots+q^{2(p-1)}\right)\left[V_{0}\right]=0$. Thus, we identify it with the ring $\mathbb{Z}[q] /\left(1+q^{2}+\cdots+q^{2(p-1)}\right)$, also denoted $\mathbb{O}_{p}$ :

$$
\mathbb{O}_{p}:=\mathbb{Z}[q] /\left(1+q^{2}+\cdots+q^{2(p-1)}\right) \cong K_{0}(H-\underline{\text { fmod }}) .
$$


For $p \neq 2$, the polynomial $1+q^{2}+\cdots+q^{2(p-1)}$ decomposes into the product of the $p$-th and $2 p$-th cyclotomic polynomials:

$$
1+q^{2}+\cdots+q^{2(p-1)}=\Psi_{p}(q) \Psi_{2 p}(q) .
$$

This is readily seen from the relation $\left(1-q^{2}\right) \Psi_{p}(q) \Psi_{2 p}(q)=1-q^{2 p}$, which in turn also shows that

$$
\Psi_{2 p}(q)=\sum_{i=0}^{p-1}(-q)^{i}=\Psi_{p}(-q) .
$$

It follows that $\mathbb{Z}[q] /\left(\Psi_{p}(q)\right) \cong \mathbb{Z}[q] /\left(\Psi_{2 p}(q)\right)$. These rings, which are also known as the rings of the $p$-th and $2 p$-th cyclotomic integers, are isomorphic to each other and usually denoted by $\mathcal{O}_{p}$ and $\mathcal{O}_{2 p}$ :

$$
\mathcal{O}_{p}:=\mathbb{Z}[q] /\left(\Psi_{p}(q)\right), \quad \mathcal{O}_{2 p}:=\mathbb{Z}[q] /\left(\Psi_{2 p}(q)\right), \quad \mathcal{O}_{p} \cong \mathcal{O}_{2 p}
$$

By mapping $q$ to a primitive $p$-th root of unity $\zeta_{p} \in \mathbb{C}$ (resp. a primitive $2 p$-th root of unity $\zeta_{2 p} \in \mathbb{C}$ ), we identify $\mathcal{O}_{p} \cong \mathbb{Z}\left[\zeta_{p}\right]$ (resp. $\mathcal{O}_{2 p} \cong \mathbb{Z}\left[\zeta_{2 p}\right]$ ), and the corresponding fields of fractions $\mathbb{Q}[q] /\left(\Psi_{p}(q)\right) \cong \mathbb{Q}\left[\zeta_{p}\right]$ (resp. $\left.\mathbb{Q}[q] /\left(\Psi_{2 p}(q)\right) \cong \mathbb{Q}\left[\zeta_{2 p}\right]\right)$. Equation (15) induces quotient maps

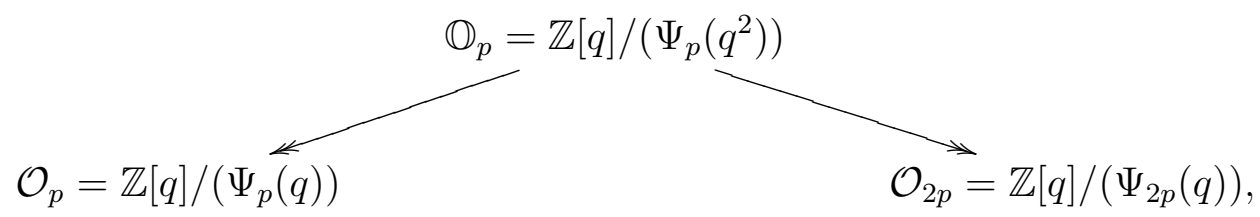

with the injective product map $\mathbb{O}_{p} \longrightarrow \mathcal{O}_{p} \times \mathcal{O}_{2 p}$. Inverting $2 \in \mathbb{Z}$, the product map induces an isomorphism $\mathbb{O}_{p}\left[\frac{1}{2}\right] \cong \mathcal{O}_{p}\left[\frac{1}{2}\right] \times \mathcal{O}_{2 p}\left[\frac{1}{2}\right]$. We have $\mathbb{O}_{p} \otimes_{\mathbb{Z}\left[q, q^{-1}\right]} \mathcal{O}_{p} \cong \mathcal{O}_{p}$ and $\mathbb{O}_{p} \otimes_{\mathbb{Z}\left[q, q^{-1}\right]} \mathcal{O}_{2 p} \cong \mathcal{O}_{2 p}$. Likewise, $\mathbb{O}_{p} \otimes_{\mathbb{Z}\left[q, q^{-1}\right]} \mathbb{F}_{p} \cong \mathbb{F}_{p}$, via the homomorphism $\mathbb{Z}\left[q, q^{-1}\right] \longrightarrow \mathbb{F}_{p}$ taking $q$ to 1 .

Notation 2.7. In what follows, we will refer to the symbol $[U] \in K_{0}(H-\underline{\text { fmod }}) \cong \mathbb{O}_{p}$ of an object $U \in H-\underline{\mathrm{fmod}}$ as the $\mathbb{O}_{p}$-dimension of $U$, while $[U]_{p}:=[U](\bmod p) \in \mathbb{F}_{p}$ as its $\mathbb{F}_{p}$-dimension.

Our choice of having $\operatorname{deg}(\partial)=2$ rather than 1 is mainly due to the standard grading of the KLR algebras $R(\nu)$ (see Section 4) and other objects that categorify quantum groups and their representations. This results in a slight annoyance that $K_{0}(H-\underline{\text { mod }})$ is no longer an integral domain, as shown by equation (15) (c.f. [18, Section 3]). However, this choice of degree has an additional, if rather modest, merit, as we will see shortly that the existence of balanced indecomposable $H$-modules makes the cohomology theories more symmetric with respect to duality. 
Cohomology of a $p$-complex. When $p=2$, a graded $H$-module $U$ is just a complex of $\mathbb{k}$-vector spaces with a degree two differential, and has associated cohomology groups $\mathrm{H}(U)=\operatorname{ker} \partial / \operatorname{im} \partial$.

One possible analogue of these groups for larger $p$ 's is given by the following construction. For each $0 \leq k \leq p-2$ form the graded vector space

$$
\mathrm{H}_{/ k}(U)=\operatorname{Ker}\left(\partial^{k+1}\right) /\left(\operatorname{Im}\left(\partial^{p-k-1}\right)+\operatorname{Ker}\left(\partial^{k}\right)\right) .
$$

The original $\mathbb{Z}$-grading of $U$ gives a decomposition

$$
\mathrm{H}_{/ k}(U)=\bigoplus_{i \in \mathbb{Z}} \mathrm{H}_{/ k}^{i}(U)
$$

The differential $\partial$ induces a map $\mathrm{H}_{/ k} \longrightarrow \mathrm{H}_{/ k-1}$, also denoted $\partial$, which takes $\mathrm{H}_{/ k}^{i}(U)$ to $\mathrm{H}_{/ k-1}^{i+2}(U)$. Define the slash cohomology of $U$ as

$$
\mathrm{H}_{/}(U)=\bigoplus_{k=0}^{p-2} \mathrm{H}_{/ k}(U)
$$

Let also

$$
\mathrm{H}^{i}(U):=\bigoplus_{k=0}^{p-2} \mathrm{H}_{k}^{i}(U)
$$

We have the decompositions

$$
\mathrm{H}_{/}(U)=\bigoplus_{i \in \mathbb{Z}} \mathrm{H}^{i}(U)=\bigoplus_{k=0}^{p-2} \mathrm{H}_{/ k}(U)=\bigoplus_{i \in \mathbb{Z}} \bigoplus_{k=0}^{p-2} \mathrm{H}_{/ k}^{i}(U)
$$

$\mathrm{H}_{/}(U)$ is a bigraded $\mathbb{k}$-vector space, equipped with an operator $\partial$ of bidegree $(2,-1)$, $\partial: \mathrm{H}_{/ k}^{i} \longrightarrow \mathrm{H}_{/ k-1}^{i+2}$. The /-grading (slash-grading) is nontrivial only in degrees between 0 and $p-2$. Necessarily $\partial^{p-1}=0$.

Forgetting about the /-grading gives us a graded vector space $\mathrm{H}_{/}(U)$ with the differential $\partial$, which we can view as a graded $H$-module. $\mathrm{H}_{/}(U)$ is isomorphic to $U$ in the stable category $H-\underline{\bmod }$, and we can decompose

$$
U \cong \mathrm{H}_{/}(U) \oplus P(U)
$$

in the abelian category of $H$-modules, where $P(U)$ is a maximal projective direct summand of $U$. The cohomology group $\mathrm{H}_{/}(U)$, viewed as an $H$-module, does not contain any direct summand isomorphic to a free $H$-module.

This assignment $U \mapsto \mathrm{H}_{/}(U)$ is functorial in $U$ and can be viewed as a functor $H-\bmod \longrightarrow H-\underline{\bmod }$ or as a functor $H-\underline{\bmod } \longrightarrow H-\underline{\bmod }$; the latter functor is isomorphic to the identity functor. 
Example 2.8. When $p=3$,

$$
\mathrm{H}(U)=\left(\operatorname{Ker}\left(\partial^{2}\right) /(\operatorname{Im} \partial+\operatorname{Ker} \partial)\right) \oplus \operatorname{Ker}(\partial) / \operatorname{Im}\left(\partial^{2}\right),
$$

with the differential going from the first summand to the second.

The following way to think about these cohomology groups is helpful. Since a $p$ complex splits into a direct sum of indecomposable $p$-complexes $V_{i}\{j\}$, we can reduce our consideration to the cohomology of these complexes. We depict $V_{i}$ by an oriented chain with $i+1$ vertices and $i$ oriented edges; each vertex denotes a basis vector in the corresponding weight space of $V_{i}$ and oriented edges - the action of $\partial$.

$$
\stackrel{0}{\circ} \longrightarrow \stackrel{1}{\circ} \longrightarrow \circ \stackrel{\cdots}{\longrightarrow} \circ \longrightarrow \stackrel{i}{\circ}
$$

For an even simpler picture, keep vertices only and switch to balanced indecomposables. Assemble diagrams for $\widetilde{V}_{p-1}, \widetilde{V}_{p-2}, \ldots, \widetilde{V}_{0}$ into a column to form a triangle with $p$ points on each side, and let $\widetilde{V}=\widetilde{V}_{p-1} \oplus \widetilde{V}_{p-2} \oplus \cdots \oplus \widetilde{V}_{0}$.

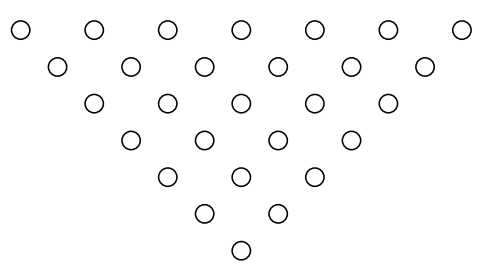

We think of points in the triangle diagram as basis elements of $\widetilde{V}$, with the differential acting one step to the right. The $k$-th slash cohomology groups $\mathrm{H}_{/ k}(\widetilde{V})$ for $0 \leq k \leq p-2$ pick out the terms in the $k$-th diagonal in the triangle, except for the vertex in the top row (the top row corresponds to the unique projective indecomposable module in the direct sum), for the total of $p-1-k$ vertices.

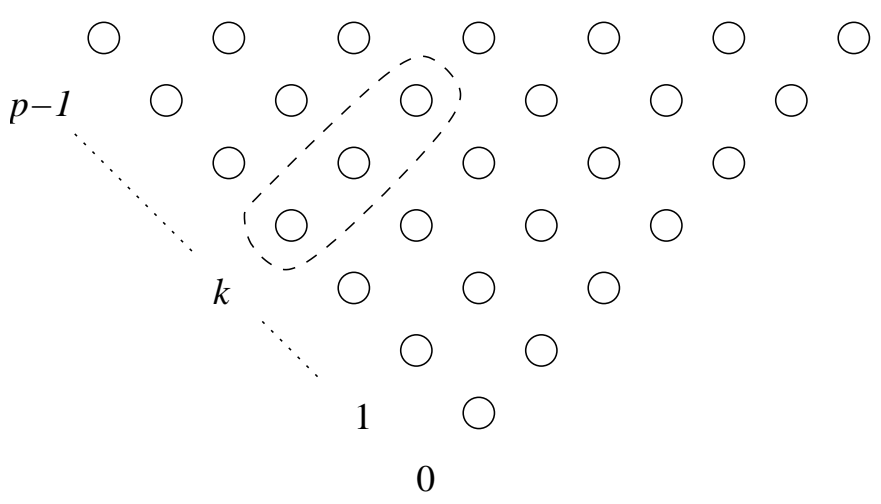


The zeroth cohomology $\mathrm{H}_{/ 0}(\widetilde{V})$ collects vertices on the southwest-northeast edge of the triangle save for the rightmost top one. The last nonzero term $\mathrm{H}_{/ p-2}(\widetilde{V})$ picks out only one vertex, below and to the right of the upper left one.

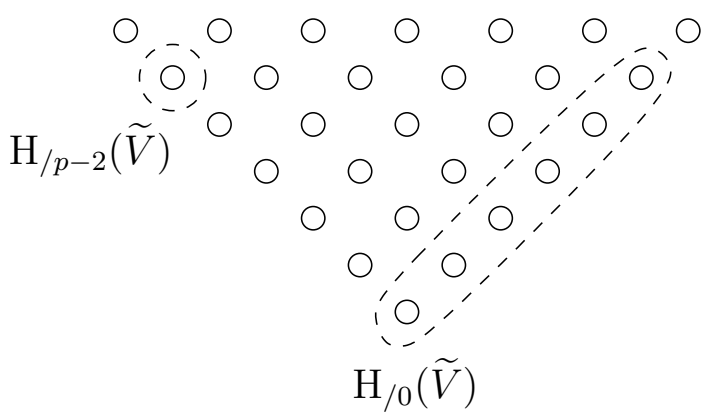

Our diagram of dots in a triangle has a $y$-axis symmetry, and it is natural to introduce a second family of cohomology groups, obtained by "reflecting" the original groups about the symmetry axis. We call these the backslash cohomology and define them by

$$
\mathrm{H}_{\backslash k}=\left(\operatorname{Im}\left(\partial^{k}\right) \cap \operatorname{Ker}\left(\partial^{p-k-1}\right)\right) / \operatorname{Im}\left(\partial^{k+1}\right) .
$$

The dotted curve below surrounds basis elements of $\mathrm{H}_{\backslash k}(\widetilde{V})$.

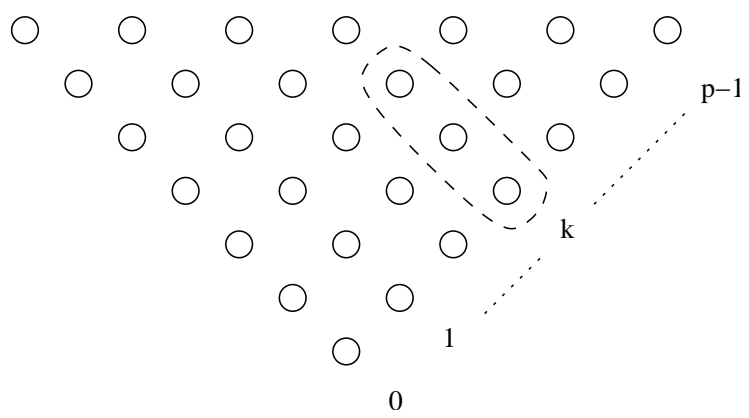

We have

$$
\mathrm{H}_{/ k}(U)^{*} \cong \mathrm{H}_{\backslash k}\left(U^{*}\right), \quad \mathrm{H}_{\backslash k}(U)^{*} \cong \mathrm{H}_{/ k}\left(U^{*}\right),
$$

where "*" denotes the graded dual $H$-module.

In comparison, the original cohomology groups of Mayer [33, 34] assigned to a $p$ complex and generalized by Kapranov [15] and Sarkaria [42, 43], see also Dubois-Violette [7], are

$$
{ }_{k} \mathrm{H}=\operatorname{Ker} \partial^{k} / \operatorname{Im} \partial^{p-k}
$$

for $1 \leq k \leq p-1$. 
Of course ${ }_{1} \mathrm{H}(U)=\mathrm{H}_{/ 0}(U)$ for any $p$-complex $U$, but the two types of groups are otherwise different. Diagrammatically, the $k$-th Mayer cohomology ${ }_{k} \mathrm{H}$ picks out a parallelogram with $k$ vertices in the northwest direction and $p-k$ in the northeast direction:

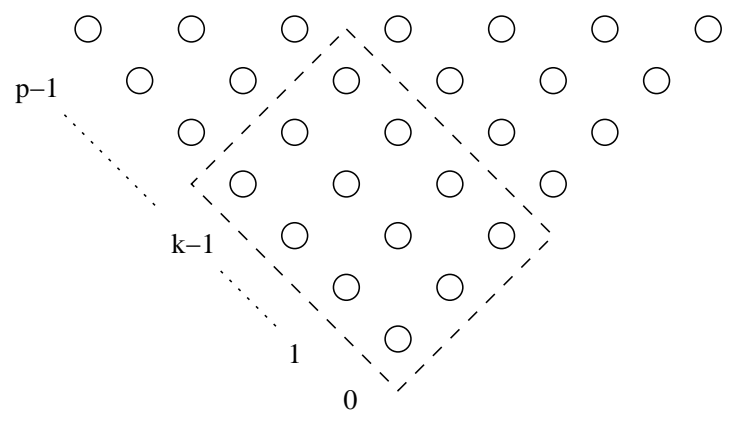

Thus, $\mathrm{H}_{/ k-1}$ is the cokernel of the natural homomorphism ${ }_{k-1} \mathrm{H} \longrightarrow{ }_{k} \mathrm{H}$, while the kernel is precisely the backslash cohomology group $\mathrm{H}_{\backslash p-k}$. See the picture below.

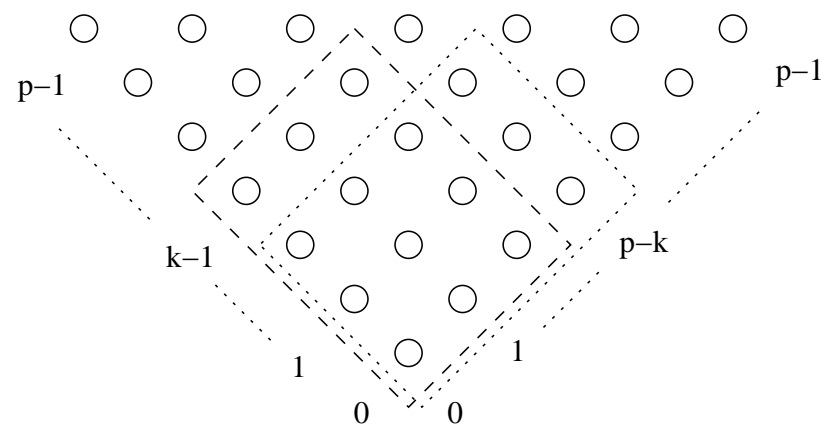

Overall, the various cohomology groups fit into exact sequences

$$
0 \longrightarrow \mathrm{H}_{\backslash p-k}(U) \longrightarrow{ }_{k-1} \mathrm{H}(U) \longrightarrow{ }_{k} \mathrm{H}(U) \longrightarrow \mathrm{H}_{/ k-1}(U) \longrightarrow 0
$$

functorially in $U$.

The two types of groups together with connecting homomorphisms carry the same amount of information; either one determines the isomorphism type of the $p$-complex in the stable category. They differ only by packaging. The new packaging via (back)slash cohomology describes a minimal (unique up to isomorphism) representative of a $p$ complex $U$ in the stable category as $\mathrm{H}_{/}(U)$. Jonsson's "train cohomology" groups [14] pick out single vertices on the longest anti-diagonal (northwest-southeast direction) in the triangle diagram. 


\section{$2.2 p$-DG algebras and $p$-DG modules}

p-DG algebras. By analogy with DG algebras and DG modules [2, Section 10.1], we now define their counterparts: $p$-DG algebras and $p$-DG modules. Recall that $\mathbb{k}$ is a field of characteristic $p>0$.

Definition 2.9. A $p$-DG algebra $A$ is graded $\mathbb{k}$-algebra $A$ equipped with a $\mathbb{k}$-linear derivation (also called a differential) $\partial_{A}: A \longrightarrow A$ of degree 2 (i.e. $\partial_{A}: A^{k} \longrightarrow A^{k+2}$ for all $k \in \mathbb{Z}$ ), which satisfies the $p$-nilpotency condition

$$
\partial_{A}^{p}=0
$$

and the Leibniz rule

$$
\partial_{A}(a b)=\partial_{A}(a) b+a \partial_{A}(b)
$$

for any elements $a, b \in A$.

When the algebra $A$ is generated by a set of elements $\left\{a_{i} \mid i \in I\right\}$, to check that $\partial^{p}=0$ on the whole algebra, it is enough to check that $\partial^{p}\left(a_{i}\right)=0$ for all $i \in I$, since $\partial^{p}(a b)=\partial^{p}(a) b+a \partial^{p}(b)$ (in characteristic $p, \partial^{p}$ is a derivation if $\partial$ is). A $\partial$-stable graded two-sided ideal $I$ of $A$ gives rise to a $p$-DG algebra $A / I$. The direct product $A \times B$ and the tensor product $A \otimes B$ of $p$-DG algebras $A$ and $B$ are again $p$-DG algebras with the obvious gradings and differentials.

Definition 2.10. A left $p$-DG module $\left(M, \partial_{M}\right)$ over a $p$-DG algebra $A$ is a graded left $A$-module $M$ with a $\mathbb{k}$-linear endomorphism $\partial_{M}$ of degree 2 (i.e. $\partial_{M}: M^{k} \longrightarrow M^{k+2}$ for all $k \in \mathbb{Z}$ ) such that it is $p$-nilpotent:

$$
\partial_{M}^{p}=0
$$

and for any $a \in A, m \in M$

$$
\partial_{M}(a m)=\partial_{A}(a) m+a \partial_{M}(m)
$$

A morphism of (left) $p$-DG-modules is a morphism of (left) $A$-modules of degree zero which commutes with the differentials. We will denote the category of left $p$-DG modules by $A_{\partial}-\bmod$, or, alternatively, by $(A, \partial)$-mod. Likewise, a right $p$ - $D G$ module $N$ over $A$ consists of a right $A$-module $N$ equipped with a $\mathbb{k}$-linear endomorphism $\partial_{N}$ of degree 2 such that:

$$
\partial_{N}^{p}=0
$$

and for any $a \in A, n \in N$

$$
\partial_{N}(n a)=\partial_{N}(n) a+n \partial_{A}(a)
$$

We will omit the subscripts in $\partial_{A}, \partial_{M}, \partial_{N}$ whenever no confusion can arise. 
Remark 2.11 ( $p$-DG algebra as $H$-module algebra). A $p$-DG algebra is a graded $H$ module algebra and can also be described as $H^{\prime}$-comodule algebra, for a suitable infinitedimensional Hopf algebra $H^{\prime}$ related to the dual of $H$. The $p$-derivation on $A$ allows us to define the smash product ring $A_{\partial}$ as follows. As a $\mathbb{k}$-vector space, $A_{\partial} \cong A \otimes H$, and the multiplication on $A_{\partial}$ is given by $\left(a_{1} \otimes 1\right) \cdot\left(a_{2} \otimes 1\right)=\left(a_{1} a_{2}\right) \otimes 1,\left(1 \otimes h_{1}\right) \cdot\left(1 \otimes h_{2}\right)=$ $1 \otimes\left(h_{1} h_{2}\right),\left(a_{1} \otimes 1\right)\left(1 \otimes h_{1}\right)=a_{1} \otimes h_{1}$ for any $a_{1}, a_{2} \in A, h_{1}, h_{2} \in H$, and by the rule for commuting elements of the form $a \otimes 1,1 \otimes \partial$ :

$$
(1 \otimes \partial)(a \otimes 1)=(a \otimes 1)(1 \otimes \partial)+\partial(a) \otimes 1 .
$$

Note that $1 \otimes H \subset A_{\partial}$ is a subalgebra. Moreover, since $A, H$ are compatibly graded, i.e. $\operatorname{deg}(\partial(a))=\operatorname{deg}(a)+2$ for any homogeneous $a \in A$, the commutator equation is homogeneous so that $A_{\partial}$ is graded. The category of left (resp. right) $p$-DG modules is equivalent to the category of graded left (resp. right) $A_{\partial}$-modules. This explains our choice of notation for the category of left (resp. right) $p$-DG modules above and shows that it is an abelian category with arbitrary coproducts.

The algebras $A_{\partial}$ are examples of smash product algebras, see [35, 39] for instance.

If $A=\mathbb{k}$ is the ground field, a $p$-DG module over $A$ is just a $p$-complex of $\mathbb{k}$-vector spaces. A 2-DG algebra is the same as a differential graded algebra over a field of characteristic two, with the differential of degree two rather than one.

\section{The homotopy category.}

Definition 2.12. Two morphisms of $p$-DG modules $f, g: M \longrightarrow N$ in $A_{\partial}-\bmod$ are said to be homotopic if there exists a morphism $h$ of $A$-modules of degree $2-2 p$ such that:

$$
f-g=\sum_{i=0}^{p-1} \partial_{N}^{p-1-i} \circ h \circ \partial_{M}^{i} .
$$

It is readily seen that the collection of all morphisms that are homotopic to zero (nullhomotopic morphisms) forms an ideal in $A_{\partial}$-mod. A $p$-DG module $M$ is called contractible if $\operatorname{Id}_{M}$ is null-homotopic. The homotopy category $\mathcal{C}(A, \partial)$ of $p$-DG complexes is the categorical quotient of $A_{\partial}-\bmod$ by the ideal of null-homotopic morphisms.

We often abbreviate $\mathcal{C}(A, \partial)$ to $\mathcal{C}(A)$ when the context is clear.

The tensor product of a $p$-complex $V$ and $p$-DG module $M$ is naturally a $p$-DG module. The $A$ action on $M$ is extended to the whole of $V \otimes M$ by letting it act trivially on $V$, and $\partial$ acts by $\partial(x \otimes m):=\partial(x) \otimes m+x \otimes \partial(m)$ for any $x \in V$ and $m \in M$. This gives a bifunctor

$$
\otimes: H-\bmod \times A_{\partial}-\bmod \longrightarrow A_{\partial}-\bmod , \quad(V, M) \mapsto V \otimes M .
$$

The tensor product descends to homotopy categories:

$$
\otimes: H-\underline{\bmod } \times \mathcal{C}(A) \longrightarrow \mathcal{C}(A)
$$


and equips $\mathcal{C}(A)$ with a "triangulated module-category" structure over $H$ - $\underline{\bmod }$. The shift functor on $\mathcal{C}(A)$ is inherited from $H-\underline{\bmod }$.

Definition 2.13. The shift endo-functor $[1]$ on $\mathcal{C}(A)$ is defined by

$$
M \mapsto \widetilde{V}_{p-2}\{-p\} \otimes M .
$$

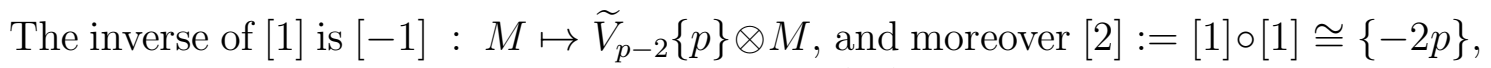
with the isomorphisms coming from the map $\tilde{\iota}$ of (11).

Definition 2.14. Let $\underline{u}: M \longrightarrow N$ be a morphism in $\mathcal{C}(A)$, and $u: M \longrightarrow N$ be a lift of $\underline{u}$ in $A_{\partial}-$ mod. Let $\bar{C}_{u}$ be the push-out of $u: M \longrightarrow N$ and $\lambda_{M}: M \longrightarrow H \otimes M$, so that it fits into the following commutative diagram:

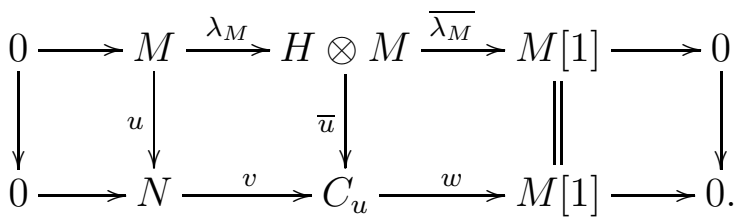

The standard exact triangle associated with $\underline{u}$ in $\mathcal{C}(A)$ is the sextuple

$$
M \stackrel{\underline{u}}{\longrightarrow} N \stackrel{\underline{v}}{\longrightarrow} C_{u} \stackrel{\underline{w}}{\longrightarrow} M[1]
$$

where the underlined morphisms denote the image of the corresponding un-underlined ones in $\mathcal{C}(A)$. We say that a sextuple $M \stackrel{u}{\longrightarrow} N \stackrel{v}{\longrightarrow} W \stackrel{w}{\longrightarrow} M[1]$ is an exact triangle in $\mathcal{C}(A)$ if it is isomorphic to a standard one in $\mathcal{C}(A)$.

Proposition 2.15. The homotopy category $\mathcal{C}(A)$ together with the shift functor [1] and the class of exact triangles described above is triangulated.

Proof. See [18, Theorem 1].

The class of exact triangles in $\mathcal{C}(A)$ can also be characterized as consisting of the images of some short exact sequences of $A_{\partial}$-modules.

Lemma 2.16. Let $0 \longrightarrow M \stackrel{u}{\longrightarrow} N \stackrel{v}{\longrightarrow} W \longrightarrow 0$ be a short exact sequence of $A_{\partial^{-}}$ modules which splits as a sequence of A-modules. Then associated with it there is an exact triangle

$$
M \stackrel{\underline{u}}{\longrightarrow} N \stackrel{\underline{v}}{\longrightarrow} W \stackrel{\underline{w}}{\longrightarrow} M[1]
$$

in the homotopy category.

Proof. See [39, Lemma 4.3]. 
The derived category. Since $A_{\partial}$ contains $H$ as a subalgebra, the natural forgetful functor from the category of $p$-DG modules to the base category of $p$-complexes allows us to define the cohomologies $\mathrm{H}_{\backslash}(M), \mathrm{H}_{/}(M)$ of a $p$-DG module $M$ to be the cohomologies of the $p$-complex $M$. A morphism $u: M \longrightarrow N$ of $p$-DG modules naturally induces a morphism of the underlying $p$-complexes, and gives rise to morphisms on cohomologies:

$$
u_{\backslash}: \mathrm{H}_{\backslash}(M) \longrightarrow \mathrm{H}_{\backslash}(N), \quad u_{/}: \mathrm{H}_{/}(M) \longrightarrow \mathrm{H}_{/}(N) .
$$

This allows us to define the notion of quasi-isomorphisms.

Definition 2.17. A morphism of $p$-DG modules $u: M \longrightarrow N$ is called a quasiisomorphism if it induces an isomorphism of the underlying $p$-complexes up to homotopy. This is equivalent to either of the following conditions on cohomology:

a) $u_{\backslash}: \mathrm{H}_{\backslash}(M) \cong \mathrm{H}_{\backslash}(N)$,

b) $u_{/}: \mathrm{H}_{/}(M) \cong \mathrm{H}_{/}(N)$.

A $p$-DG module $M$ is called acyclic if $0 \longrightarrow M$, or equivalently $M \longrightarrow 0$, is a quasiisomorphism.

Notice that a contractible $p$-DG module is automatically acyclic but not vice versa. However, these notions coincide for the ground field $\mathbb{k}$ viewed as a $p$-DG algebra.

It follows from the definition that a morphism of $p$-DG modules which is homotopic to a quasi-isomorphism is also a quasi-isomorphism, leading to a well-defined notion of quasi-isomorphism in the homotopy category $\mathcal{C}(A)$.

Proposition 2.18. Quasi-isomorphisms in $\mathcal{C}(A)$ constitute a localizing class.

Proof. This is Proposition 4 of [18, Section 1]. We also refer the reader to [12, Section III.2] for the standard notion of a localizing class and definition of localization with respect to a localizing class.

Definition 2.19. Let $A$ be a $p$-DG algebra. We define the derived category $\mathcal{D}(A, \partial)$ of $p$-DG modules over $A$ to be the localization of $\mathcal{C}(A, \partial)$ with respect to the class of quasi-isomorphisms. When no confusion can arise, we will write $\mathcal{D}(A)$ for short.

$\mathcal{D}(A)$ inherits a natural triangulated structure from that of $\mathcal{C}(A)$. The class of exact triangles in $\mathcal{D}(A)$ consists of sextuples $M \stackrel{u}{\longrightarrow} N \stackrel{v}{\longrightarrow} W \stackrel{w}{\longrightarrow} M[1]$ that are isomorphic to the images of exact triangles in $\mathcal{C}(A)$. Moreover, it also inherits a triangulated module-category structure over $H-\underline{\text { fmod }}$ from that of $\mathcal{C}(A)$.

Lemma 2.20. Let $0 \longrightarrow M \stackrel{u}{\longrightarrow} N \stackrel{v}{\longrightarrow} W \longrightarrow 0$ be a short exact sequence of $A_{\partial-}$ modules. Then associated with it there is an exact triangle

$$
M \stackrel{\underline{u}}{\longrightarrow} N \stackrel{\underline{v}}{\longrightarrow} W \stackrel{\underline{w}}{\longrightarrow} M[1]
$$

in the derived category. 
Proof. Omitted. This together with the explicit construction of $\underline{w}$ is Lemma 4.4 of [39, Section 4].

In one very particular situation the derived category is easy to determine, namely when it is equivalent to the zero category.

Proposition 2.21. Let $A$ be a p-DG algebra. The following statements are equivalent.

i) $\mathcal{D}(A) \cong 0$.

ii) A is acyclic.

iii) There exists an element $a \in A$ such that $\partial^{p-1}(a)=1$.

iv) There exists an element $b \in A$ such that $\partial(b)=1$.

Furthermore, if $b$ is central in $A$, then $\mathcal{C}(A) \cong 0$.

Proof. $i) \Rightarrow i i) . A \cong 0$.

$i i) \Rightarrow$ iii). Since $\partial(1)=0$ and $A$ is acyclic, Proposition $2.2 v)$ implies that there is an element $a$ with $\partial^{p-1}(a)=1$.

$i i i) \Rightarrow i v)$. Take $b=\partial^{p-2}(a)$.

$i v) \Rightarrow i)$. To do this we use the following elementary identity: if $D$ and $L$ are $\mathbb{k}$-linear maps on a vector space satisfying $[D, L]=\mathrm{Id}$, then

$$
\sum_{i=0}^{p-1} D^{p-1-i} L^{p-1} D^{i}=-\mathrm{Id} .
$$

If this is proven, let $L=L_{b}$ be the left multiplication on any $p$-DG module $M$ by the element $b$. Then $\left[\partial_{M}, L_{b}\right]=\partial(b)=\operatorname{Id}_{M}$ and $i$ ) follows by taking the homotopy to be $-\left(L_{b}\right)^{p-1}$. Now we prove the identity. Let $\operatorname{ad}_{D}(L):=[D, L]$, and note that $\operatorname{ad}_{D}$ is a derivation on the space of all linear operators. On one hand,

$$
\begin{aligned}
\left(\operatorname{ad}_{D}\right)^{p-1}\left(L^{p-1}\right) & =\left(\operatorname{ad}_{D}\right)^{p-2}\left((p-1) L^{p-2} \operatorname{ad}_{D}(L)\right)=\left(\operatorname{ad}_{D}\right)^{p-2}\left((p-1) L^{p-2} \cdot \mathrm{Id}\right) \\
& =\operatorname{ad}_{D}^{p-3}\left((p-1)(p-2) L^{p-3} \operatorname{ad}_{D}(L)\right) \cdots=(p-1) ! \operatorname{Id} \equiv-\operatorname{Id}(\bmod p) .
\end{aligned}
$$

On the other hand, expanding the iterated commutators directly results in

$$
\begin{aligned}
\operatorname{ad}_{D}^{p-1}\left(L^{p-1}\right) & =\left[D,\left[D, \cdots,\left[D, L^{p-1}\right] \cdots\right]\right]=\sum_{i=0}^{p-1}(-1)^{i}\left(\begin{array}{c}
p-1 \\
i
\end{array}\right) D^{i} L^{p-1} D^{p-1-i} \\
& =\sum_{i=0}^{p-1} D^{i} L^{p-1} D^{p-1-i}
\end{aligned}
$$

The claimed identity follows.

For the last statement, notice that the proof of $i v) \Rightarrow i$ ) says that $-\left(L_{b}\right)^{p-1}$ on any $p$-DG module $M$ is a homotopy between $\operatorname{Id}_{M}$ and $0_{M}$, and thus any $M$ is acyclic. If $b$ is moreover central, then $L_{b}^{p-1}$ is an $A$-module map. The result follows. 
Morphism spaces as cohomology. Similar to the usual DG case, the morphism space in the homotopy category $\mathcal{C}(A)$ is the degree zero part of a certain cohomology $p$-complex RHOM.

First off, recall that in the abelian category $A_{\partial}$-mod, the morphism space between two $p$-DG modules $M, N$ can be computed as

$$
\begin{aligned}
\operatorname{Hom}_{A_{\partial}}(M, N) & \cong\left\{u \in \operatorname{Hom}_{A}(M, N) \mid \partial \circ u-u \circ \partial=0\right\} \\
& \cong\left\{u \in \operatorname{Hom}_{A}(M, N) \mid \partial \cdot u=0\right\} \\
& \cong \operatorname{Ker}\left(\partial: \operatorname{HOM}_{A}^{0}(M, N) \longrightarrow \operatorname{HOM}_{A}^{2}(M, N)\right),
\end{aligned}
$$

where "." denotes the $H$-action on $\mathrm{HOM}_{A}$, and $\mathrm{HOM}_{A}^{2}(M, N)$ stands for the space of $A$-module maps homogeneous of degree two. Similarly by Definition 2.12 , there are canonical isomorphisms of $\mathbb{k}$-vector spaces

$$
\begin{aligned}
\operatorname{Hom}_{C(A)}(M, N) & \cong \frac{\operatorname{Hom}_{A_{\partial}}(M, N)}{\partial^{p-1} \cdot \operatorname{Hom}_{A}^{2-2 p}(M, N)} \\
& \cong \frac{\operatorname{Ker}\left(\partial: \operatorname{HOM}_{A}^{0}(M, N) \longrightarrow \operatorname{HOM}_{A}^{2}(M, N)\right)}{\operatorname{Im}\left(\partial^{p-1}: \operatorname{HOM}_{A}^{2-2 p}(M, N) \longrightarrow \operatorname{HOM}_{A}^{0}(M, N)\right)} \\
& \cong \mathrm{H}_{/ 0}^{0}\left(\operatorname{HOM}_{A}(M, N)\right),
\end{aligned}
$$

i.e. this is just the degree zero part of the slash (or backslash) cohomology of the $p$-complex $\operatorname{HOM}_{A}(M, N)$. It forgets a lot of information about the total $p$-complex $\mathrm{HOM}_{A}(M, N)$ even if $A_{\partial}$-module maps of all degrees are taken into account:

$$
\operatorname{HOM}_{\mathcal{C}(A)}(M, N):=\bigoplus_{i \in \mathbb{Z}} \operatorname{Hom}_{\mathcal{C}(A)}(M, N\{i\})=\mathrm{H}_{/ 0}\left(\operatorname{HOM}_{A}(M, N)\right) .
$$

We summarize the above discussion in the following proposition, whose proof in the more general case of module-algebras over any finite dimensional Hopf algebras can be found in [39, Section 5].

Proposition 2.22. The following diagram commutes

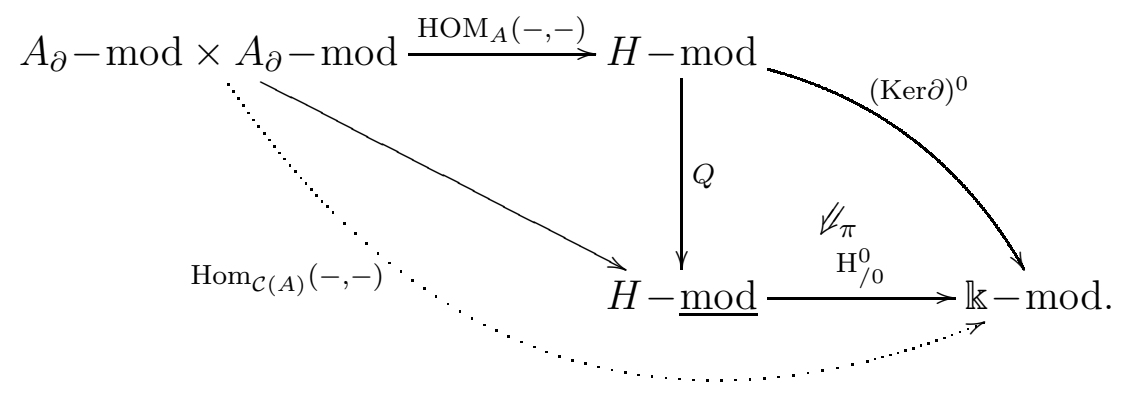

Here $Q$ is the natural quotient functor which is the identity on object 2 from the abelian category of p-complexes to its homotopy category, and $\pi$ is the natural transformation which on a p-complex $V$ is given by $\pi(V):(\operatorname{Ker} \partial)^{0}(V):=\operatorname{Ker}\left(\left.\partial\right|_{V^{0}}\right) \longrightarrow \mathrm{H}_{/ 0}^{0}(V)$.

\footnotetext{
${ }^{2}$ We usually just omit writing it when the context is clear.
} 
Cofibrant modules and bar resolution. In the DG case, morphism spaces between "nice enough" objects in the derived category coincide with the morphism spaces of the same objects in the homotopy category. The analogous result also holds in the $p$-DG case, which we now make precise.

Following Keller [17], we make the following definitions.

Definition 2.23. Let $A$ be a $p$-DG algebra and $P$ a $p$-DG module over $A$.

i) We say that $P$ is cofibrant if for any surjective quasi-isomorphism of $p$-DG modules $M \rightarrow N$, the induced map of graded $\mathbb{k}$-vector spaces $\operatorname{HOM}_{A_{\partial}}(P, M) \longrightarrow$ $\mathrm{HOM}_{A_{\partial}}(P, N)$ is surjective.

ii) We say that $P$ satisfies property $(\mathrm{P})$ if the following two conditions holds:

(P 1) There is an exhaustive (possibly infinite) filtration of $P$ by $A_{\partial}$-submodules:

$$
0=F_{-1} \subset F_{0} \subset F_{1} \subset \cdots \subset F_{r} \subset F_{r+1} \subset \cdots \subset P
$$

Here being exhaustive means that $P=\cup_{r=0}^{\infty} F_{r}$.

(P 2) The associated graded modules of the filtration $F_{r+1} / F_{r}$ for all $r \in \mathbb{N}$ are isomorphic to (possibly infinite) direct sums of free $A$-modules of the form $A\{s\}, s \in \mathbb{Z}$.

The cofibrance property is equivalent to requiring that the induced map on the homogeneous degree zero part of $\mathrm{HOM}_{A_{\partial}}$, which is just $\operatorname{Hom}_{A_{\partial}}(P, M) \longrightarrow \operatorname{Hom}_{A_{\partial}}(P, N)$, be surjective, since $M \rightarrow N$ is a surjective quasi-isomorphism if and only if $M\{r\} \rightarrow$ $N\{r\}$ for any $r \in \mathbb{Z}$ is.

We list the main properties of these two types of modules without proof. The reader can find the proofs in the more general framework of hopfological algebra in [39, Section $6]$.

Proposition 2.24. Let $A$ be a p-DG algebra. Cofibrant and property $(P)$ modules over A enjoy the following properties:

i) Property $(P)$ modules are cofibrant.

ii) If $P$ is cofibrant and $M$ is acyclic, then $\operatorname{HOM}_{A}(P, M)$ is a contractible $p$-complex. In particular $\operatorname{Hom}_{\mathcal{C}(A)}(P, M)=0$.

iii) A p-DG module $P$ is cofibrant if and only if $P$ is projective as an A-module, and for any acyclic $p$-DG $A$-module $M, \operatorname{HOM}_{A}(P, M)$ is an acyclic $p$-complex.

iv) If $P$ is cofibrant and $M$ is any $p$-DG module, then there is an isomorphism of $\mathbb{k}$-vector spaces $\operatorname{Hom}_{\mathcal{C}(A)}(P, M) \cong \operatorname{Hom}_{\mathcal{D}(A)}(P, M)$.

v) A p-DG module is cofibrant if and only if it is a direct summand of a property (P) module in the abelian category $A_{\partial}$-mod. 
Proof. Omitted. See the results $6.4-6.10$ of [39].

The following theorem states that there are always "enough" property $(\mathrm{P})$ modules.

Theorem 2.25 (Bar resolution). Let $A$ be a $p$-DG algebra. For any $p-D G$ module $M$, there is a surjective quasi-isomorphism of $p$ - $D G$ modules

$$
\mathbf{p}(M) \rightarrow M,
$$

where $\mathbf{p}(M)$ satisfies property $(P)$. Moreover, the assignment $M \mapsto \mathbf{p}(M)$ is functorial in $M$.

Proof. See Theorem 6.6 and Corollary 6.7 of [39].

We will refer to the functorial cofibrant module $\mathbf{p}(M)$ as the bar resolution of $M$.

Corollary 2.26. Let $\mathcal{C} \mathcal{F}(A)$ (resp. $\mathcal{P}(A)$ ) denote the full-subcategory of $\mathcal{C}(A)$ consisting of cofibrant (resp. property $(P))$ modules. Then the composition of functors

$$
\begin{gathered}
\mathcal{C F}(A) \subset \mathcal{C}(A) \stackrel{Q}{\longrightarrow} \mathcal{D}(A), \\
\text { (resp. } \mathcal{P}(A) \subset \mathcal{C}(A) \stackrel{Q}{\longrightarrow} \mathcal{D}(A)),
\end{gathered}
$$

where $Q$ is the localization functor, is an equivalence of categories.

Compact modules and Grothendieck groups. When working with the abelian or derived category of modules over some ring, allowing infinitely generated modules makes the Grothendieck group of the module category zero. One needs to restrict the size of (projective) modules to define the Grothendieck group. Similar consideration applies in the $p$-DG context, and an appropriate size restriction on $p$-DG modules is the compactness condition.

Definition 2.27. Let $A$ be a $p$-DG algebra. An object $M \in \mathcal{D}(A)$ is called compact if the functor $\operatorname{Hom}_{\mathcal{D}(A)}(M,-): \mathcal{D}(A) \longrightarrow \mathbb{k}-\bmod$ commutes with arbitrary direct sums.

For instance, the module $A\{r\}$ is compact for any $r \in \mathbb{Z}$.

Proposition 2.28. Let $A$ be a $p-D G$ algebra. The derived category $\mathcal{D}(A)$ is generated by the set of compact objects $\{A\{r\} \mid r \in \mathbb{Z}\}$, in the sense that if $M \in \mathcal{D}(A)$ satisfies

$$
\operatorname{Hom}_{\mathcal{D}(A)}(A\{r\}[s], M)=0
$$

for all $r, s \in \mathbb{Z}$, then $M \cong 0$ in $\mathcal{D}(A)$.

Proof. Omitted. See [39, Proposition 7.6].

The general machinery of Ravenel-Neeman can be applied to the compactly generated category $\mathcal{D}(A)$ to characterize the class of compact objects in it. 
Definition 2.29. A $p$-DG module $M \in A_{\partial}$-mod is called a finite cell module if there is a finite exhaustive filtration of $A_{\partial}$-modules

$$
0=F_{-1} \subset F_{0} \subset \cdots \subset F_{r-1} \subset F_{r} \subset \cdots \subset F_{N}=M
$$

such that the associated graded modules $F_{r} / F_{r-1}$ for all $0 \leq r \leq N$ are isomorphic, as $A$-modules, to finite direct sums of the free $A$-modules $A\{s\}$ for various $s \in \mathbb{Z}$.

In other words, finite cell modules are just property $(\mathrm{P})$ modules that are finitely generated as $A$-modules.

Theorem 2.30 (Characterization of compact modules). The compact objects in $\mathcal{D}(A)$ are those which are isomorphic in the derived category to a direct summand of a finite cell module.

Proof. Omitted. See Theorem 7.14 and Corollary 7.15 of [39].

Informally, we think of the notions introduced in this section as analogues of the usual ring theoretic concepts below. In the comparison table, $R$ stands for some ring, "f.g." is short for "finitely generated", and the arrows indicate inclusion relations. Dotted line surrounds types of objects in the abelian category $A_{\partial}$-mod, while the bottom right arrow indicates that any finite cell module is a compact object in the derived category $\mathcal{D}(A)$.

$$
R-\bmod \quad A_{\partial}-\bmod
$$
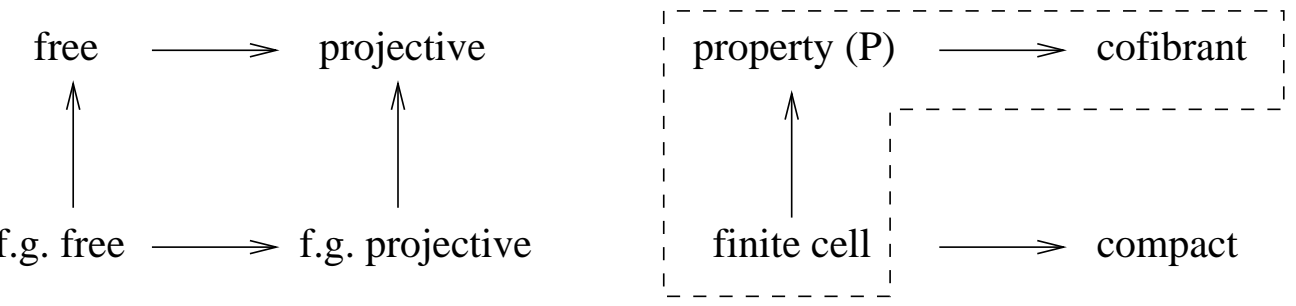

Denote by $\mathcal{D}^{c}(A)$ the strictly full subcategory of $\mathcal{D}(A)$ consisting of compact objects. The above theorem implies that $\mathcal{D}^{c}(A)$ is Karoubian, or equivalently idempotent complete. In the simplest case $A \cong \mathbb{k}$ there is an equivalence of categories $H-\underline{\text { mod }} \cong \mathcal{D}^{c}(\mathbb{k})$.

Definition 2.31. The Grothendieck group $K_{0}\left(\mathcal{D}^{c}(A)\right.$ ) of a $p$-DG algebra $A$ (or $K_{0}(A)$ if no confusion can arise) is the abelian group generated by the symbols of the isomorphism classes of objects in $\mathcal{D}^{c}(A)$, subject to the relations that

$$
[M]=[L]+[N]
$$

whenever there is an exact triangle $L \longrightarrow M \longrightarrow N \longrightarrow L[1]$ in $\mathcal{D}^{c}(A)$.

The exact tensor bi-functor $\otimes: H-\underline{\bmod } \times \mathcal{D}(A) \longrightarrow \mathcal{D}(A)$ restricts to an exact bi-functor

$$
\otimes: H-\underline{\mathrm{fmod}} \times \mathcal{D}^{c}(A) \longrightarrow \mathcal{D}^{c}(A),
$$

which in turn equips $K_{0}(A)$ with a module structure over the ring $\mathbb{O}_{p} \cong K_{0}(H-\underline{\mathrm{fmod}})$. 
Derived hom and tensor product. Observe that if $A$ is a $p$-DG algebra, its opposite algebra $A^{\text {op }}$ equipped with the same differential $\partial$ is also a $p$-DG algebra, called the opposite $p$-DG algebra of $A$. The category of right $p$-DG modules over $A$ can be identified with that of left modules over $A^{\text {op }}$.

The category of $p$-DG algebras has a natural monoidal structure as follows. If $A$ and $B$ are two $p$-DG algebras, then the tensor product $p-D G$ algebra $A \otimes B$ is the usual tensor product algebra equipped with the differential $\partial(a \otimes b):=\partial(a) \otimes b+a \otimes \partial(b)$. One readily checks that this differential is compatible with the algebra structure, and furthermore $A \cong A \otimes \mathbb{k}, B \cong \mathbb{k} \otimes B$ sit inside $A \otimes B$ naturally as $p$-DG subalgebras.

Now if $A, B$ are $p$-DG algebras, a $p$-DG $(A, B)$-bimodule ${ }_{A} X_{B}$ is a left module $X$ over the $p$-DG algebra $A \otimes B^{\mathrm{op}}$. $p$-DG bimodules naturally give rise to functors on $p$-DG module categories via the hom and tensor functors

$$
\begin{gathered}
\operatorname{HOM}_{A}\left({ }_{A} X_{B},-\right): A_{\partial}-\bmod \longrightarrow B_{\partial}-\bmod , \quad{ }_{A} M \mapsto \operatorname{HOM}_{A}\left({ }_{A} X_{B},{ }_{A} M\right), \\
{ }_{A} X_{B} \otimes_{B}(-): B_{\partial}-\bmod \longrightarrow A_{\partial}-\bmod , \quad{ }_{B} N \mapsto{ }_{A} X_{B} \otimes_{B} N .
\end{gathered}
$$

Definition 2.32. Let $A, B$ be $p$-DG algebras, and ${ }_{A} X_{B}$ be a $p$-DG $(A, B)$-bimodule.

i). The derived tensor product ${ }_{A} X \otimes_{B}^{\mathbf{L}}: \mathcal{D}(B) \longrightarrow \mathcal{D}(A)$ is the composition of functors

$$
\mathcal{D}(B) \stackrel{\mathbf{p}}{\longrightarrow} \mathcal{P}(B) \stackrel{{ }_{A} X \otimes_{B}(-)}{\longrightarrow} \mathcal{C}(A) \stackrel{Q}{\longrightarrow} \mathcal{D}(A),
$$

where $Q$ is the natural localization functor.

ii). The derived hom functor $\operatorname{RHOM}\left({ }_{A} X_{B},-\right)$ is the composition of functors

$$
\mathcal{D}(A) \stackrel{\mathrm{HOM}_{A}\left(\mathbf{p}_{\mathbf{A}}(X),-\right)}{\longrightarrow} \mathcal{C}(B) \stackrel{Q}{\longrightarrow} \mathcal{D}(B),
$$

where $\mathbf{p}_{\mathbf{A}}(X)$ denotes the bar resolution of $X$ as a left $p$-DG $A$-module 3

The tensor product and hom functors satisfy the following adjunction property.

Proposition 2.33. A p-DG $(A, B)$-bimodule $X$ gives an adjunction of functors in the derived category:

$$
\operatorname{Hom}_{\mathcal{D}(A)}\left(X \otimes_{B}^{\mathbf{L}} N, M\right) \cong \operatorname{Hom}_{\mathcal{D}(B)}\left(N, \mathbf{R H O M}_{A}(X, M)\right),
$$

for any $M \in \mathcal{D}(A)$ and $N \in \mathcal{D}(B)$.

Proof. This is a special case of Proposition 8.13 in [39].

\footnotetext{
${ }^{3}$ By the construction of the bar resolution in [39, Theorem 6.6], $\mathbf{p}_{\mathbf{A}}(X)$ has a natural right $p$-DG $B$-module structure.
} 
A morphism $\mu: X \longrightarrow Y$ of $p$-DG $(A, B)$-bimodules descends to a natural transformation between the derived tensor product functors

$$
\mu^{\mathbf{L}}: X \otimes_{B}^{\mathbf{L}}(-) \Longrightarrow Y \otimes_{B}^{\mathbf{L}}(-): \mathcal{D}(B) \longrightarrow \mathcal{D}(A) .
$$

The following proposition gives a criterion on when a derived tensor functor induces an equivalence of derived categories, and when such a natural transformation is an isomorphism of functors.

Proposition 2.34. $\quad$ i) Let $X$ be a $p$ - $D G(A, B)$-bimodule and suppose it is cofibrant as a $p$-D $G$ A-module. Then $X \otimes_{B}^{\mathbf{L}}(-): \mathcal{D}(B) \longrightarrow \mathcal{D}(A)$ is an equivalence of triangulated categories if and only if the following two conditions hold:

1) The natural map $B \longrightarrow \operatorname{HOM}_{A}(X, X)$ is a quasi-isomorphism.

2) $X$, when regarded as a $p-D G A$-module, is a compact generator of $\mathcal{D}(A)$.

ii) Let $\mu: X \longrightarrow Y$ be a morphism of $p$ - $D G(A, B)$-bimodules. The natural transformation $\mu^{\mathbf{L}}: X \otimes_{B}^{\mathbf{L}}(-) \Longrightarrow Y \otimes_{B}^{\mathbf{L}}(-)$ is an isomorphism of functors if and only if $\mu: X \longrightarrow Y$ is a quasi-isomorphism of $p-D G$ bimodules.

Proof. Omitted. See Proposition 8.8 of [39].

Example 2.35. We give an example of the above proposition related to the classical Morita equivalence. Let $A$ be a $p$-DG algebra and $U$ a finite dimensional $p$-complex. Then $A \otimes U$ is a $p$-DG $A$-module which satisfies property (P) (Definition 2.23). Form the algebra $B:=\operatorname{END}_{A}(A \otimes U)^{o p} . B$ has a natural $p$-DG algebra structure where for any $f \in B$ and $x \in A \otimes U$,

$$
\partial(f)(x)=\partial(f(x))-f(\partial(x)) .
$$

Moreover, $A \otimes U$ is a $p$-DG $(A, B)$-bimodule, and we define $(A \otimes U)^{\vee}:=\operatorname{HOM}_{A}(A \otimes$ $U, A) \cong A \otimes U^{*}$, which is a $p$-DG $(B, A)$-bimodule. It is easy to see that

$$
(A \otimes U)^{\vee} \otimes_{A}(A \otimes U) \cong B, \quad(A \otimes U) \otimes_{B}(A \otimes U)^{\vee} \cong A,
$$

as $p$-DG bimodules. Therefore, the functors between abelian categories

$$
\begin{gathered}
(A \otimes U) \otimes_{B}(-): B_{\partial}-\bmod \longrightarrow A_{\partial}-\bmod , \\
(A \otimes U)^{\vee} \otimes_{A}(-): A_{\partial}-\bmod \longrightarrow B_{\partial}-\bmod ,
\end{gathered}
$$

which are mutually inverse of each other descend to equivalences of homotopy categories

$$
\begin{aligned}
&(A \otimes U) \otimes_{B}(-): \mathcal{C}(B) \longrightarrow \mathcal{C}(A), \\
&(A \otimes U)^{\vee} \otimes_{A}(-): \mathcal{C}(A) \longrightarrow \mathcal{C}(B) .
\end{aligned}
$$


It is a natural question to ask whether these functors further pass to derived equivalences. Proposition $2.34 \mathrm{i}$ ) says that if $U$ is not a contractible $p$-complex, in which case $A \otimes U$ is a compact generator of $\mathcal{D}(A)$, then

$$
(A \otimes U) \otimes_{B}^{\mathbb{L}}(-): \mathcal{D}(B) \longrightarrow \mathcal{D}(A)
$$

is an equivalence of derived categories. On the other hand, $(A \otimes U)^{\vee} \cong A \otimes U^{*}$ is not a cofibrant $B$-module if $U$ is contractible. It is cofibrant if and only if $U$ is not acyclic. When the cofibrance condition is satisfied,

$$
(A \otimes U)^{\vee} \otimes_{A}^{\mathbb{L}}(-): \mathcal{D}(A) \longrightarrow \mathcal{D}(B)
$$

is then an equivalence by Proposition 2.34 i) again.

Induction and restriction functors. A very special case of the previous discussion is when we have a map of $p$-DG algebras $\phi: B \longrightarrow A$, and the bimodule is given by ${ }_{A} X_{B}={ }_{A} A_{B}$. We will allow maps $\phi: B \longrightarrow A$ with $\partial_{B} \circ \phi=\phi \circ \partial_{A}$ which are non-unital, with $\phi\left(1_{B}\right)$ only an idempotent in $A$.

Definition 2.36. Let $\phi: B \longrightarrow A$ be a map of $p$-DG algebras.

i). The (derived) induction functor $\phi^{*}=\operatorname{Ind}_{B}^{A}$ is the derived tensor functor associated with the bimodule ${ }_{A} A_{B}$ :

$$
\phi^{*}=\operatorname{Ind}_{B}^{A}=A \otimes_{B}^{\mathbf{L}}(-): \mathcal{D}(B) \longrightarrow \mathcal{D}(A) .
$$

ii). The restriction functor $\phi_{*}=\operatorname{Res}_{B}^{A}$ is the forgetful functor via the morphism $\phi$, i.e. it takes a $p$-DG $A$-module $M$ to a $p$-DG $B$ module $\phi\left(1_{B}\right) \cdot M$ by letting $B$ act through $\phi$. It is an exact functor on $A_{\partial}-\bmod$, and therefore it descends to an exact functor

$$
\phi_{*}=\operatorname{Res}_{B}^{A}: \mathcal{D}(A) \longrightarrow \mathcal{D}(B)
$$

The restriction functor coincides with the (derived) hom functor $\operatorname{RHOM}_{A}\left(A_{B},-\right)$, since as a left $A_{\partial}$-module, $A$ satisfies property $(\mathrm{P})$, and thus for any graded $A$-module ${ }_{A} M$,

$$
\operatorname{RHOM}_{A}\left(A_{B}, M\right)=\operatorname{HOM}_{A}\left(A_{B}, M\right)={ }_{B} M .
$$

Hence by Proposition 2.33, there is an adjunction

$$
\operatorname{Hom}_{\mathcal{D}(A)}\left(\phi^{*}(N), M\right) \cong \operatorname{Hom}_{\mathcal{D}(B)}\left(N, \phi_{*}(M)\right)
$$

for any $N \in \mathcal{D}(B)$ and $M \in \mathcal{D}(A)$. It follows that $\phi^{*}\left(\mathcal{D}^{c}(A)\right) \subset \mathcal{D}^{c}(B)$ since $\phi_{*}$ obviously commutes with taking arbitrary direct sums and direct summands. Alternatively, this can be directly seen from the characterization of compact modules as finite 
cell modules (Theorem 2.30). In this language, Proposition 2.34 translates into the following important special case, which is the p-DG analogue of Theorem 10.12.5.1 of [2]. Functor $\phi_{*}$ does not necessarily preserve compact objects. It does, for instance, when $A$ has a finite $p$-DG resolution as a $(B, B)$-bimodule, or when $A$ has finite dimensional cohomology.

Theorem 2.37. Let $\phi: B \longrightarrow A$ be a morphism of $p$-DG algebras that is a quasiisomorphism. Then the induction and restriction functors

$$
\phi^{*}: \mathcal{D}(B) \longrightarrow \mathcal{D}(A), \quad \phi_{*}: \mathcal{D}(A) \longrightarrow \mathcal{D}(B)
$$

are mutually inverse equivalence of categories.

Proof. That $\phi^{*}$ is an equivalence follows from Proposition 2.34, while that of $\phi_{*}$ follows by adjunction 2.33. For details see the proof in [39, Corollary 8.17].

Corollary 2.38. If $\phi: B \longrightarrow A$ is a quasi-isomorphism of $p$-DG algebras, then $\left[\phi_{*}\right]$ : $K_{0}(A) \longrightarrow K_{0}(B)$ is an isomorphism of $\mathbb{O}_{p}$-modules.

Proof. Since being compact is a categorical concept, an equivalence of categories preserves compactness property. The result follows readily from this and Theorem 2.37.

\subsection{Special cases}

Smooth artinian algebras. A graded $\mathbb{k}$-algebra $A$ is naturally a $p$-DG algebra with the trivial differential $(\partial=0)$. An algebra $A$ is smooth if it has a finite projective resolution as an $(A, A)$-bimodules.

For the moment denote by $K_{0}^{\prime}(A)$ the usual Grothendieck group of $A$, which is a $\mathbb{Z}\left[q, q^{-1}\right]$-module, generated by symbols $[P]$ of finitely generated graded projective $A$-modules.

Proposition 2.39. The Grothendieck group of a smooth artinian algebra A, regarded as a p-DG algebra with the trivial differential, is related to the usual Grothendieck group $K_{0}^{\prime}(A)$ by

$$
K_{0}(A, \partial) \cong K_{0}^{\prime}(A) \otimes_{\mathbb{Z}\left[q, q^{-1}\right]} \mathbb{O}_{p}
$$

Proof. Omitted. See [39, Proposition 9.10].

Derivations on matrix algebras. It is well known that the space of derivations on an associative algebra $A$ modulo inner derivations (i.e. those of the form $\partial(a):=$ $[x, a]=x a-a x$, for any $a \in A$ and a fixed element $x \in A)$ is classified by the first Hochschild cohomology group $\operatorname{HH}^{1}(A)$, and this cohomology group is preserved by Morita equivalences. Thus if $A=\mathrm{M}(n, \mathbb{k})$ is the $n \times n$ matrix algebra over the ground field $\mathbb{k}$,

$$
\operatorname{Der}(A) / \operatorname{Inn}(A) \cong \mathrm{HH}^{1}(A) \cong \mathrm{HH}^{1}(\mathbb{k})=0,
$$


since on the ground field, there are no non-zero derivations. In particular this says that any derivation on the matrix algebra $\mathrm{M}(n, \mathbb{k})$ arises as taking commutator with some fixed element.

Now we specialize to matrix algebras over fields of positive characteristic $p$. We assume that $\mathbb{k}$ is algebraically closed until the end of this paragraph. Let $\partial_{J}(M):=$ $\operatorname{ad}_{J}(M)=J M-M J, \forall M \in \mathrm{M}(n, \mathbb{k})$ while $J$ is fixed. Then we have:

$$
\begin{aligned}
\left(\operatorname{ad}_{J}\right)^{p}(M) & =[J, \cdots,[J, M] \cdots]=\sum_{k=0}^{p}(-1)^{k}\left(\begin{array}{c}
p \\
k
\end{array}\right) J^{p-k} M J^{k} \\
& =J^{p} M-M J^{p}=\operatorname{ad}_{J^{p}}(M)
\end{aligned}
$$

To have $\partial_{J}^{p}=0$ on $\mathrm{M}(n, \mathbb{k})$, one needs to require $J^{p} \in Z(\mathrm{M}(n, \mathbb{k})) \cong \mathbb{k} \cdot I_{n \times n}$, say $J^{p}=\lambda \cdot I_{n \times n}$. Since $\mathbb{k}$ is algebraically closed, $\lambda$ has a $p$-th root $\mu \in \mathbb{k}$. Then we have $(J-$ $\left.\mu I_{n \times n}\right)^{p}=0$. There exists $g \in \operatorname{GL}(n, \mathbb{k})$ such that $g\left(J-\mu I_{n \times n}\right) g^{-1}=\operatorname{Diag}\left(J_{i_{1}}, \cdots, J_{i_{m}}\right)$, where $i_{1}+\cdots+i_{m}=n$ and each $J_{i_{r}}(1 \leq r \leq m)$ is the standard $i_{r} \times i_{r}$ Jordan matrix with $E_{i, i+1}=1$ and 0 everywhere else. Thus $J=g^{-1}\left(\operatorname{Diag}\left(J_{i_{1}}, \cdots, J_{i_{m}}\right)+\mu I_{n \times n}\right) g=$ $g^{-1}\left(\operatorname{Diag}\left(J_{i_{1}}, \cdots, J_{i_{m}}\right)\right) g+\mu I_{n \times n}$, and $\operatorname{ad} J=\operatorname{ad}\left(g^{-1}\left(\operatorname{Diag}\left(J_{i_{1}}, \cdots, J_{i_{m}}\right)\right) g\right)$ allows us to get rid of $\mu$ and $\lambda$. Hence we can assume from the beginning that $J^{p}=0$. Such matrices are classified up to conjugation by partitions $\left(i_{1}, \cdots, i_{m}\right) \vdash n$ with $i_{r} \leq p$ for all $1 \leq r \leq m$, with each $i_{r}$ corresponding to a Jordan block $J_{i_{r}}$ as above. In particular, $p$-nilpotent derivations on $\mathrm{M}(n, \mathbb{k})$ are classified by such partitions, and the classifications holds over any $\mathbb{k}$, no necessarily algebraically closed, so this restriction can be relaxed.

Next, we observe that $\mathrm{M}(n, \mathbb{k})$ has an obvious $\mathbb{Z}$ grading. Indeed, the multiplication rule $E_{i, j} E_{k, l}=\delta_{j, k} E_{i, l}$ gives us a $\mathbb{Z}$-grading $\operatorname{deg}\left(E_{i, j}\right)=j-i$. The space of degree $r$ homogeneous elements consists of matrices whose non-zero entries are concentrated on the shifted $r$-th diagonal, i.e. the span of $E_{i, j}, j-i=r$. Thus the space of homogeneous derivations on $\mathrm{M}(n, \mathbb{k})$ of degree one with respect to this grading coincide with the shifted first diagonal $\oplus_{0 \leq i \leq n-1} \mathbb{k} E_{i, i+1}$.

A toy model. We examine the graded matrix algebra $\mathrm{M}(n, \mathbb{k})$ as above, with the derivation of the simplest Jordan type: for any $M \in \mathrm{M}(n, \mathbb{k})$,

$$
\partial_{n}(M):=\left[J_{n}, M\right]
$$

where $J_{n}=\sum_{i=1}^{n-1} E_{i, i+1}$ has only one Jordan block. Necessarily $n \leq p$. It is easy to see that $\partial_{n}\left(E_{k, n}\right)=E_{k-1, n}=E_{k-1, n} E_{n, n}$, for $1 \leq k \leq n$. To make the degrees match our conventions, set $\operatorname{deg}\left(E_{i, j}\right)=2(j-i)$ so that $\operatorname{deg}\left(\partial_{n}\right)=2$.

Lemma 2.40. Let $R$ be a ring and $e \in R$ an idempotent such that $R=R e R:=$ $\left\{\sum_{r, r^{\prime}}\right.$ rer $\left.^{\prime} \mid r, r^{\prime} \in R\right\}$. Then $R$ is Morita equivalent to the ring eRe.

Proof. $(R e) \otimes_{e R e}(-): e R e-\bmod \longrightarrow R-\bmod$, and $(e R) \otimes_{R}(-): R-\bmod \longrightarrow e R e-\bmod$ are readily seen to be mutually inverse functors between the two categories. 
We will apply this lemma in the situation when $R=\mathrm{M}(n, \mathbb{k})_{\partial_{n}}$ is the smash product algebra (2.11).

Proposition 2.41. When $1 \leq n \leq p$, the algebra $\mathrm{M}(n, \mathbb{k})_{\partial_{n}}$ is Morita equivalent to $H$. Proof. $\mathrm{M}(n, \mathbb{k})_{\partial_{n}}$ has a basis $\left\{E_{i, j} \partial_{n}^{r} \mid 1 \leq i, j \leq n, 0 \leq r \leq p-1\right\}$. Thus

$$
\begin{aligned}
\mathrm{M}(n, \mathbb{k})_{\partial_{n}} \cdot E_{n, n} \cdot \mathrm{M}(n, \mathbb{k})_{\partial_{n}} & \supset\left\{\sum_{i, j} E_{i, n} E_{n, n} E_{n, j} \partial_{n}^{r} \mid 1 \leq i, j \leq n, 0 \leq r \leq p-1\right\} \\
= & \left\{\sum_{i, j} E_{i, j} \partial_{n}^{r} \mid 1 \leq i, j \leq n, 0 \leq r \leq p-1\right\} \\
= & \mathrm{M}(n, \mathbb{k})_{\partial_{n}},
\end{aligned}
$$

and the above lemma applies. We conclude that $\mathrm{M}(n, \mathbb{k})_{\partial_{n}}$ is Morita equivalent to the subring $E_{n, n} \cdot \mathrm{M}(n, \mathbb{k})_{\partial_{n}} \cdot E_{n, n}$. This subring is spanned by elements of the form $\left\{E_{n, i} \partial_{n}^{r} E_{n, n} \mid 1 \leq i \leq n, 0 \leq r \leq p-1\right\}$. We claim that in fact it has as a basis $\left\{E_{n, n} \partial_{n}^{r} E_{n, n} \mid 0 \leq r \leq p-1\right\}$. This is readily checked using the commutator relation

$$
\left[\partial_{n}^{k}, E_{n, n}\right]=\sum_{l=1}^{k}\left(\begin{array}{l}
k \\
l
\end{array}\right) E_{n-l, n} \partial_{n}^{k-l},
$$

where we set $E_{s, n}=0$ if $s \leq 0$. The formula follows by an easy induction and the $\partial_{n}$ action on $E_{k, n}$ above. Finally, the same commutator relation also implies that, for any $0 \leq r, s \leq p-1$,

$$
\left(E_{n, n} \partial_{n}^{r} E_{n, n}\right)\left(E_{n, n} \partial_{n}^{s} E_{n, n}\right)=E_{n, n} \partial_{n}^{r+s} E_{n, n},
$$

which in turn shows that $E_{n, n} \cdot \mathrm{M}(n, \mathbb{k})_{\partial_{n}} \cdot E_{n, n}$ has the same ring structure as $H$. The proposition follows.

The abelian category of graded $\mathrm{M}(n, \mathbb{k})_{\partial_{n}}$-modules is Krull-Schmidt. As an immediate application of the previous result, the indecomposable modules are classified as follows.

Corollary 2.42. When $1 \leq n \leq p$, any indecomposable graded $\mathrm{M}(n, \mathbb{k})_{\partial_{n}}$-module is isomorphic to precisely one of the form $\widetilde{V}_{i}\{m\} \otimes \mathbb{k}^{n}$ for $i \in\{0,1, \cdots p-1\}$ and $m \in \mathbb{Z}$, where $\mathbb{k}^{n}=\oplus_{j=1}^{n} \mathbb{k} E_{j, n}$ is the column $p$-DG module and the differential acts by $\partial_{n}\left(E_{j, n}\right)=$ $E_{j-1, n}$.

Proof. Follows directly from the proof of Lemma 2.40 and the previous proposition.

In particular, if $n=p$, the column module is acyclic, and the corollary implies that all $p$-DG modules over $\mathrm{M}(p, \mathbb{k})$ are acyclic. Thus $\mathcal{D}\left(\mathrm{M}(p, \mathbb{k}), \partial_{p}\right) \cong 0$. In fact an easy computation shows that

$\partial_{p}\left(\sum_{i=1}^{p-1} i E_{i+1, i}\right)=\left[\left(\begin{array}{cccccc}0 & 1 & & & & \\ & 0 & 1 & & & \\ & & 0 & 1 & & \\ & & & \ddots & \ddots & \\ & & & & 0 & 1 \\ & & & & & 0\end{array}\right),\left(\begin{array}{cccccc}0 & & & & & \\ 1 & 0 & & & & \\ & 2 & 0 & & & \\ & & \ddots & \ddots & & \\ & & p-2 & 0 & \\ & & & p-1 & 0\end{array}\right)\right]=I_{p}$, 
so that Proposition 2.21 iii) applies.

Proposition 2.43. If $1 \leq n \leq p-1$, the column $p$-DG module $\mathbb{k}^{n}$ over the $p$-DG algebra $\left(\mathrm{M}(n, \mathbb{k}), \partial_{n}\right)$ is a compact cofibrant generator of $\mathcal{D}(\mathrm{M}(n, \mathbb{k}))$.

Proof. Let $K$ be any indecomposable acyclic $p$-DG module over $\left(\mathrm{M}(n, \mathbb{k}), \partial_{J}\right)$. By our classification of indecomposable modules, $K \cong \mathbb{k}^{n} \otimes V_{p-1}\{m\}$ for some $m \in \mathbb{Z}$. Thus $\mathrm{HOM}_{\mathrm{M}(n, \mathbb{k})}\left(\mathbb{k}^{n}, K\right)=\mathrm{HOM}_{\mathrm{M}(n, \mathbb{k})}\left(\mathbb{k}^{n}, \mathbb{k}^{n}\right) \otimes V_{p-1}\{m\} \cong V_{p-1}\{m\}$, which is a contractible $p$-complex. Therefore Proposition 2.24 iii) implies that $\mathbb{k}^{n}$ is cofibrant. The compactness of $\mathbb{k}^{n}$ is clear since it is finite dimensional. Finally, it is a generator since the free left p-DG module $\mathrm{M}(n, \mathbb{k})$ has a filtration by column submodules $F_{1} \subset F_{2} \subset F_{3} \subset \cdots \subset$ $F_{n-1} \subset F_{n}=\mathrm{M}(n, \mathbb{k})$, such that the quotients $F_{r} / F_{r-1} \cong \mathbb{k}^{n}\{2(r-1)\}$. The result follows.

Remark 2.44. When $1 \leq n \leq p-1$, the iterated extension of $\mathrm{M}(n, \mathbb{k})$ in the abelian category $\mathrm{M}(n, \mathbb{k})_{\partial_{n}}-\bmod$ gives rise to a convolution diagram in $\mathcal{D}(\mathrm{M}(n, \mathbb{k}))$, using Lemma 2.20,

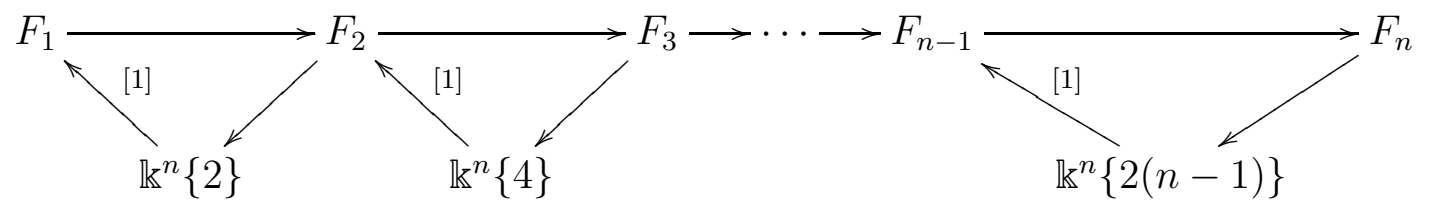

where $F_{1} \cong \mathbb{k}^{n}$ and $F_{n} \cong \mathrm{M}(n, \mathbb{k})$. Since $\mathcal{D}(\mathrm{M}(n, \mathbb{k}))$ is generated by $\mathrm{M}(n, \mathbb{k})$ (Proposition 2.28), it follows from this diagram that $\mathbb{k}^{n}$ is a generator of $\mathcal{D}(\mathrm{M}(n, \mathbb{k}))$, and $[\mathrm{M}(n, \mathbb{k})]=\left(1+q^{2}+\cdots+q^{2(n-1)}\right)\left[\mathbb{k}^{n}\right]$ in $K_{0}(\mathrm{M}(n, \mathbb{k}))$.

Corollary 2.45. If $1 \leq n \leq p-1$, the functor

$$
\mathbb{k}^{n} \otimes_{\mathbb{k}}(-): \mathcal{D}(\mathbb{k}) \longrightarrow \mathcal{D}(\mathrm{M}(n, \mathbb{k}))
$$

is an equivalence of triangulated categories. Consequently, $K_{0}(\mathrm{M}(n, \mathbb{k})) \cong \mathbb{O}_{p}$.

Proof. The cofibrance of $\mathbb{k}^{n}$ allows us to pass from derived tensor product to underived tensor product. The result follows directly from the previous result and Proposition 2.34.

The method generalizes with essentially no change to the case when the differential on $\mathrm{M}(n, \mathbb{k})$ has more than one Jordan block. The above Proposition 2.43 and Corollary 2.45 hold as long as all Jordan blocks are of size less or equal to $p$ and there is at least one Jordan block of size strictly less than $p$. If all Jordan blocks are of size $p$, the algebra is acyclic and the derived category collapses to zero. 


\section{The $p$-DG nilHecke algebra}

\section{$3.1 \quad p$-derivations on the nilHecke algebra}

A $p$-derivation on the polynomial ring. Define the derivation $\partial$ on the ring of polynomials $\mathbb{k}[x] \operatorname{deg}(x)=2$, by $\partial(x)=x^{2}$. This implies $\partial\left(x^{m}\right)=m x^{m+1}$, and thus $\partial^{p}(x)=0$, therefore making $\mathbb{k}[x]$ into an $H$-module algebra. The inclusion $\mathbb{k} \subset \mathbb{k}[x]$ is an isomorphism in the stable category $H-\underline{\bmod }$, since under the action of $\partial$ the algebra of polynomials decomposes into the trivial representation $\mathbb{k}$ of $H$ and free modules $H\{2+2 p k\}$ spanned by $x^{1+p k}, x^{2+p k}, \cdots, x^{p(k+1)}$ for $k \in \mathbb{N}$ (recall that we adopt the convention $\mathbb{N}=\{0,1,2, \ldots\})$.

Let $\operatorname{Pol}_{n}=\mathbb{k}\left[x_{1}, \ldots, x_{n}\right]$ be the ring of polynomials in $n$ variables, with $\operatorname{deg}\left(x_{i}\right)=2$. Define the derivation $\partial$ on $\mathrm{Pol}_{n}$ by $\partial\left(x_{i}\right)=x_{i}^{2}$. Viewed as a graded $H$-module, $\mathrm{Pol}_{n}$ is the tensor product of $n$ copies of the module $\mathbb{k}[x]$. Since the latter is isomorphic to $V_{0}$ in $H-\underline{\bmod }$, the inclusion $V_{0} \subset \mathrm{Pol}_{n}$ taking $V_{0}$ to $\mathbb{k} \cdot 1$ is an isomorphism in $H$ - $\underline{\bmod }$.

We denote by $\mathcal{P}_{n}$ the space $\operatorname{Pol}_{n}$ with the grading shifted down by $\frac{n(n-1)}{2}$, viewed as a graded left $\mathrm{Pol}_{n}$-module,

$$
\mathcal{P}_{n}:=\operatorname{Pol}_{n}\left\{\frac{n(1-n)}{2}\right\} .
$$

The generator 1 of $\mathcal{P}_{n}$ lives in degree $\frac{n(1-n)}{2}$, and $\mathcal{P}_{n}=\operatorname{Pol}_{n} \cdot 1$. We call graded module $\mathcal{P}_{n}$ the balanced free $\mathrm{Pol}_{n}$-module, and sometimes denote its elements $f \cdot 1$ instead of just $f$.

A $p$-DG module structure on the balanced module $\mathcal{P}_{n}$ is determined by $\partial(1)=g_{\alpha} \cdot 1$, where $\alpha=\left(\alpha_{1}, \ldots, \alpha_{n}\right) \in \mathbb{k}^{n}, g_{\alpha}=\sum \alpha_{i} x_{i}$ is a linear function in $x_{i}$ 's, viewed as an element of $\mathcal{P}_{n}$. Then for any $f \in \operatorname{Pol}_{n}$,

$$
\partial(f \cdot 1)=\partial(f) \cdot 1+f \cdot \partial(1)=\left(\partial(f)+f g_{\alpha}\right) \cdot 1
$$

The condition $\partial^{p}=0$ translates into $\alpha_{i} \in \mathbb{F}_{p}$, i.e. the coefficients being residues mod $p$. We denote this $p$-DG module structure on $\mathcal{P}_{n}$ by $\mathcal{P}_{n}(\alpha)$ and its generator 1 by $1_{\alpha}$ to stress their dependence on $\alpha=\left(\alpha_{1}, \ldots, \alpha_{n}\right) \in \mathbb{F}_{p}^{n}$. In what follows, to each $\alpha_{t} \in \mathbb{F}_{p}$ we assign the corresponding element of $\mathbb{N}$, via the inclusion $\{0,1, \ldots, p-1\} \subset \mathbb{N}$, and denote it by $\alpha_{t}$ (as long as no confusion is possible).

$\mathrm{Up}$ to an overall grading shift, $\mathcal{P}_{n}(\alpha)$ is isomorphic (as a $p$-DG $\mathrm{Pol}_{n}$-module) to the ideal inside the $p$-DG algebra $\mathrm{Pol}_{n}$ generated by the element $x_{1}^{\alpha_{1}} \cdots x_{n}^{\alpha_{n}}$. As an $H$ module, $\mathcal{P}_{n}(\alpha)$ decomposes into the tensor product of modules $\mathcal{P}_{1}\left(\alpha_{t}\right)$ over $\mathbb{k}\left[x_{t}\right]$ for $t$ ranging from 1 to $n$. The $H$-module $\mathcal{P}_{1}(\alpha), \alpha \in \mathbb{F}_{p}$ contains the submodule spanned by $1_{\alpha}, x_{1} 1_{\alpha}, \ldots, x_{1}^{p-\alpha} 1_{\alpha}$ for $\alpha \in \mathbb{F}_{p}^{*}$ and by $1_{\alpha}$ for $\alpha=0$, and the inclusion of this submodule into $\mathcal{P}_{1}(\alpha)$ is a quasi-isomorphism. Thus, $\mathcal{P}_{1}(0) \cong V_{0}$, and $\mathcal{P}_{1}(\alpha) \cong V_{p-\alpha}$ for $\alpha \neq 0$. The module $\mathcal{P}_{1}(\alpha)$ is contractible if and only if $\alpha=1$, and, more generally, $\mathcal{P}_{n}(\alpha)$ is a contractible $H$-module if and only if at least one of the coefficients $\alpha_{t}$ of the linear function $g_{\alpha}$ is 1 . 
The induced action on symmetric functions. The symmetric group $S_{n}$ acts on $\mathrm{Pol}_{n}$ by permuting the variables. Denote by $\mathrm{Sym}_{n}$ the subalgebra of $\mathrm{Pol}_{n}$ consisting of $S_{n}$-invariant functions, $\operatorname{Sym}_{n}=\mathrm{Pol}_{n}^{S_{n}}$. The derivation $\partial$ commutes with the action of $S_{n}$ and restricts to a derivation on $\mathrm{Sym}_{n}$. This subalgebra of symmetric functions is free with generators being elementary symmetric polynomials $e_{1}, e_{2}, \ldots, e_{n}$, where $e_{m}$ is the sum of $x_{i_{1}} \cdots x_{i_{m}}$ over all subsets $\left\{i_{1}, \ldots, i_{m}\right\}$ of $\{1,2, \ldots, n\}$ of cardinality $m$ :

$$
e_{m}=\sum_{1 \leq i_{1}<\cdots<i_{m} \leq n} x_{i_{1}} \cdots x_{i_{m}} .
$$

Later, when varying the number $n$ of variables, we will denote $e_{m}$ by $e_{m}^{(n)}$ to stress the dependence on $n$. For instance $e_{1}^{(1)}=x_{1}$, and $e_{2}^{(3)}=x_{1} x_{2}+x_{1} x_{3}+x_{2} x_{3}$.

We have the following explicit formula describing the differential on the elementary symmetric functions.

Lemma 3.1. The derivation $\partial$ acts on the elementary symmetric functions as follows:

$$
\partial\left(e_{m}\right)=e_{1} e_{m}-(m+1) e_{m+1} \quad(m<n), \quad \partial\left(e_{n}\right)=e_{1} e_{n} .
$$

Proof. Consider the following generating function over $\mathbb{Z}[t]$ for the elementary symmetric functions (we set $e_{0}:=1$ )

$$
\prod_{i=1}^{n}\left(1+x_{i} t\right)=\sum_{m=0}^{n} e_{m} t^{m},
$$

and let $\partial$ act on it as a $\mathbb{Z}[t]$-linear derivation which is determined by $\partial\left(x_{i}\right)=x_{i}^{2}$. Then differentiating both sides gives us

$$
\begin{aligned}
\sum_{m=0}^{n} \partial\left(e_{m}\right) t^{m} & =\sum_{i=1}^{n}\left(x_{i}^{2} t \cdot \prod_{j \neq i}\left(1+x_{j} t\right)\right) \\
& =\sum_{i=1}^{n}\left(\left(x_{i}^{2} t+x_{i}\right) \cdot \prod_{j \neq i}\left(1+x_{j} t\right)-x_{i} \cdot \prod_{j \neq i}\left(1+x_{j} t\right)\right) \\
& =\sum_{i=1}^{n}\left(x_{i} \prod_{j=1}^{m}\left(1+x_{j} t\right)\right)-\frac{\partial}{\partial t} \prod_{j=1}^{n}\left(1+x_{j} t\right) \\
& =e_{1} \cdot \prod_{j=1}^{n}\left(1+x_{j} t\right)-\frac{\partial}{\partial t} \prod_{j=1}^{n}\left(1+x_{j} t\right) \\
& =e_{1} \cdot \sum_{m=0}^{n} e_{m} t^{m}-\sum_{m=-1}^{n-1}(m+1) e_{m+1} t^{m} .
\end{aligned}
$$

Comparing coefficients of $t$ on both sides gives the claimed formula.

As a graded $\mathrm{Sym}_{n}$-module, $\mathrm{Pol}_{n}$ is free of rank $n$ ! with a basis $\left\{x_{1}^{b_{1}} x_{2}^{b_{2}} \cdots x_{n-1}^{b_{n-1}} \mid 0 \leq\right.$ $\left.b_{i} \leq n-i\right\}$. More generally, for any fixed permutation $s$ of $\{0,1, \cdots, n-1\}$, the set $\left\{x_{1}^{\overline{b_{1}}} x_{2}^{b_{2}} \cdots x_{n}^{b_{n}} \mid 0 \leq a_{i} \leq s(i-1)\right\}$ is a basis of the module.

When $n<p$, the group algebra $\mathbb{k}\left[S_{n}\right]$ is semisimple, and the $S_{n}$-representation $\mathrm{Pol}_{n}$ decomposes into a direct sum of its isotypic components $\operatorname{Pol}_{n, \lambda}$, over all partitions $\lambda$ of $n$, corresponding to irreducible representations $L_{\lambda}$ of $S_{n}$. The algebra of invariants $\operatorname{Sym}_{n}=\operatorname{Pol}_{n}^{S_{n}}=\operatorname{Pol}_{n,(n)}$ is identified with the summand corresponding to the trivial 
representation $L_{(n)}$. There is only one possibility for the corresponding direct sum decomposition of the trivial representation $V_{0}$ in the stable category $\left(V_{0} \cong \operatorname{Pol}_{n}\right.$ in $H-\underline{\bmod })$.

Corollary 3.2. When $n<p$, the natural inclusion $V_{0} \subset \mathrm{Sym}_{n}$ is an equivalence in the stable category $H-\underline{\bmod }$, while $\mathrm{Pol}_{n, \lambda}$ is contractible for any partition $\lambda \neq(n)$.

When $n=p$, the elementary symmetric function $e_{p}=x_{1} x_{2} \ldots x_{p}$ has the property that $e_{p}^{p}$ is in the kernel but not in the image of $\partial$, as easily seen from Lemma 3.1. The inclusion $V_{0}\left\{2 p^{2}\right\} \subset \mathrm{Pol}_{n}$ taking a basis vector of $V_{0}$ to $e_{p}^{p}$ realizes $V_{0}\left\{2 p^{2}\right\}$ as a direct summand of the $H$-module $\mathrm{Pol}_{p}$. More generally, for any $a_{1}, \ldots, a_{p} \geq 0$ the inclusion $V_{0}\left\{2 p\left(a_{1}+\cdots+a_{p}\right)\right\} \subset \operatorname{Pol}_{p}$ taking a basis vector to $x_{1}^{p a_{1}} x_{2}^{p a_{2}} \ldots x_{p}^{p a_{p}}$ realizes the former as a direct summand of $\mathrm{Pol}_{p}$.

The nilHecke algebra. The nilHecke algebra $\mathrm{NH}_{n}$ on $n$ strands over the field $\mathbb{k}$, see [23, 25], has generators $x_{1}, \ldots, x_{n}$ and $\delta_{1}, \ldots, \delta_{n-1}$, subject to defining relations

$$
\begin{aligned}
& \delta_{i}^{2}=0, \quad \delta_{i} \delta_{i+1} \delta_{i}=\delta_{i+1} \delta_{i} \delta_{i+1}, \quad \delta_{i} \delta_{j}=\delta_{j} \delta_{i} \quad \text { if } \quad|i-j|>1 \\
& x_{i} \delta_{j}=\delta_{j} x_{i} \text { if } j \neq i, i+1, \quad x_{i} x_{j}=x_{j} x_{i} \\
& x_{i} \delta_{i}-\delta_{i} x_{i+1}=1, \quad \delta_{i} x_{i}-x_{i+1} \delta_{i}=1
\end{aligned}
$$

We often use a graphical presentation for monomials in the generators of $\mathrm{NH}_{n}$, with $x_{i}$, respectively $\delta_{i}$ depicted as a dot on the $i$-th strand and the crossing of the $i$ th and $(i+1)$-st strands:

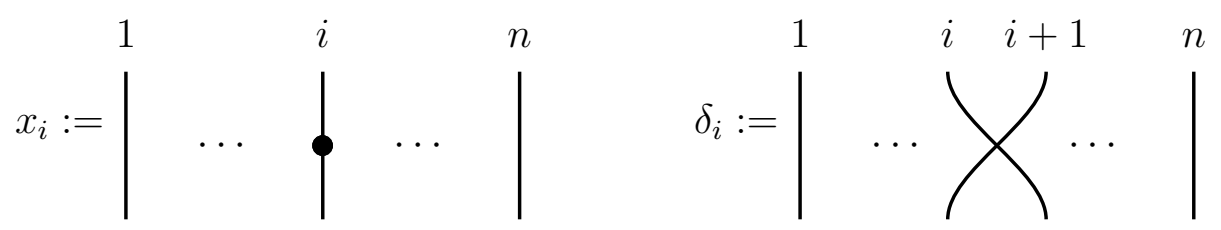

The enumerating labels on the strands will be omitted when they are clear from the context. Multiplication in $\mathrm{NH}_{n}$ is given by vertical concatenations of diagrams, with the product $x y$ represented by the picture

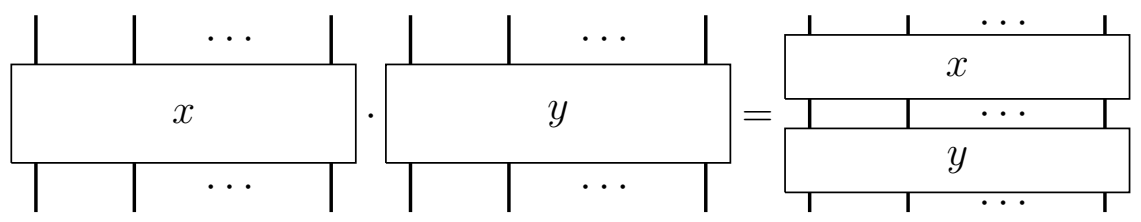

The nilHecke algebra with zero strands $\mathrm{NH}_{0} \cong \mathbb{k}$ is one-dimensional and spanned by the empty diagram $\varnothing$. The defining relations say that far away generators commute and the following diagrammatic equalities hold. 

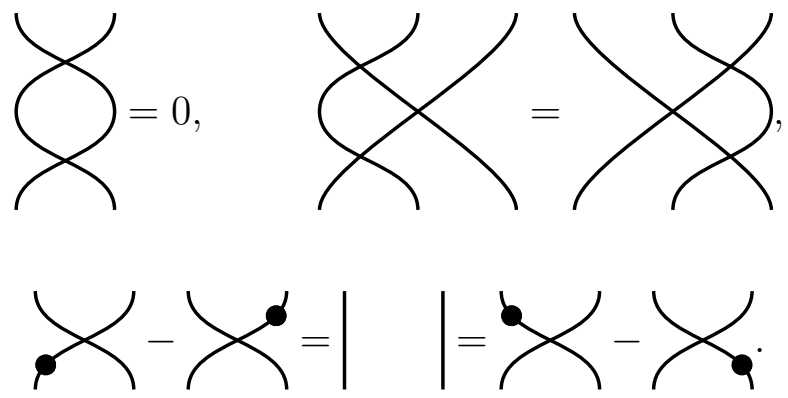

For any permutation $w$ we define $\delta_{w}:=\delta_{i_{1}} \ldots \delta_{i_{r}}$, where $w=s_{i_{1}} \ldots s_{i_{r}}$ is some reduced decomposition of $w$ into the product of elementary transpositions $s_{i}=(i, i+1)$. The nilHecke algebra has a basis with elements given by

$$
x_{1}^{b_{1}} \cdots x_{n}^{b_{n}} \delta_{w}
$$

over all $b_{1}, \ldots, b_{n} \in \mathbb{N}$ and $w \in S_{n}$. The element $x_{i}^{b}$ for some $b \in \mathbb{N}$ is denoted by a dot with a label $b$ next to it on the $i$-th strand. Each basis element can then be represented by a diagram of the composition of crossings describing permutation $w$ composed with dots on the strands above the permutation diagram.

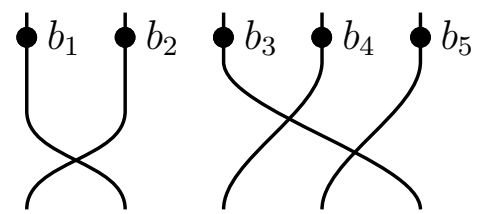

Diagrams without dots span a subalgebra of $\mathrm{NH}_{n}$ called the nilCoxeter algebra. This subalgebra, denoted $\mathrm{NC}_{n}$, has a basis $\left\{\delta_{w} \mid w \in S_{n}\right\}$.

The algebra $\mathrm{NH}_{n}$ possesses the following symmetries which are naturally described using its diagrammatic presentation. Reflecting a diagram about a horizontal axis is an algebra anti-automorphism of $\mathrm{NH}_{n}$, which we will denote by $\psi$.

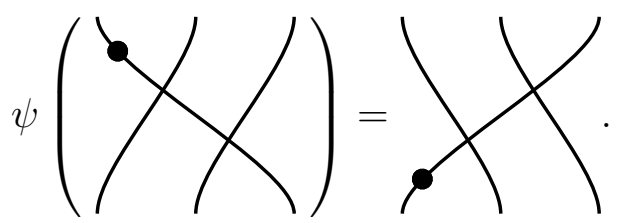

Reflecting a diagram about a vertical axis and simultaneously multiplying it by $(-1)^{s}$, where $s$ is the number of crossings in the diagram, is an algebra automorphism of $\mathrm{NH}_{n}$, which will be denoted by $\sigma$.

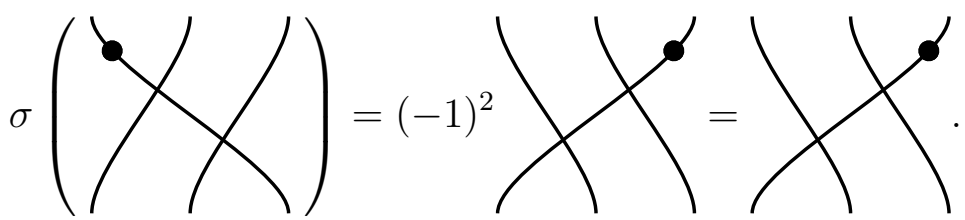


More intrinsically, the nilHecke algebra can be defined as the algebra of endomorphisms of $\mathcal{P}_{n}$ as a $\mathrm{Sym}_{n}$-module:

$$
\mathrm{NH}_{n}=\operatorname{END}_{\mathrm{Sym}_{n}}\left(\mathcal{P}_{n}\right) .
$$

The generator $x_{t}$ of $\mathrm{NH}_{n}$ acts as multiplication by $x_{t} \in \mathrm{Pol}_{n}$ on $\mathcal{P}_{n}$ (the same notation is used here for an element of $\mathrm{Pol}_{n}$ and the corresponding endomorphism of $\mathcal{P}_{n}$ ). The generator $\delta_{i}$ act as the divided difference operator

$$
\delta_{t}(f)=\frac{f-{ }^{t} f}{x_{t}-x_{t+1}},
$$

where ${ }^{t} f$ is the polynomial $f \in \mathcal{P}_{n}$ with $x_{t}, x_{t+1}$ transposed. Divided difference operators commute with multiplications by symmetric functions. It is easy to check that the relations (33)-(35) hold on these endomorphisms, resulting in a homomorphism from the algebra with these generators and relations to $\operatorname{END}_{\mathrm{Sym}_{n}}\left(\mathcal{P}_{n}\right)$. This homomorphism can be shown to be an isomorphism. We refer to $\mathcal{P}_{n}$ as the (left) balanced polynomial representation of $\mathrm{NH}_{n}$.

It is well known that $\mathcal{P}_{n}$ is a free graded module over $\mathrm{Sym}_{n}$ of rank $n$ ! and graded rank $[n]$ ! (due to balancing, the graded rank is invariant under $q \leftrightarrow q^{-1}$ symmetry). For a sequence $\beta=\left(b_{1}, \ldots, b_{n}\right)$ with $b_{k} \in \mathbb{N}$ let $x^{\beta}=x_{1}^{b_{1}} x_{2}^{b_{2}} \ldots x_{n}^{b_{n}}$. The set

$$
B_{n}=\left\{x^{\beta} \mid 0 \leq b_{t} \leq n-t, t=1, \ldots, n\right\}
$$

is a homogeneous basis of graded free $\operatorname{Sym}_{n}$-module $\mathcal{P}_{n}$. Necessarily $b_{n}=0$ for such $\beta$. Let $U_{n}=\mathbb{k}\left\{B_{n}\right\}$ be the $\mathbb{k}$-vector subspace of $\mathcal{P}_{n}$ with basis $B_{n}$. There is a canonical isomorphism

$$
U_{n} \otimes_{\mathbb{k}} \operatorname{Sym}_{n} \stackrel{\cong}{\longrightarrow} \mathcal{P}_{n}
$$

taking $x^{\beta} \otimes f$ to $f x^{\beta}$ and, via equation (38), producing an isomorphism

$$
\mathrm{NH}_{n} \cong \operatorname{END}_{\mathbb{k}}\left(U_{n}\right) \otimes_{\mathbb{k}} \operatorname{Sym}_{n} \cong U_{n} \otimes U_{n}^{*} \otimes \operatorname{Sym}_{n} .
$$

Upon ordering elements of $B_{n}$, we get an isomorphism

$$
\mathrm{NH}_{n} \cong \mathrm{M}\left(n !, \operatorname{Sym}_{n}\right),
$$

which identifies $\mathrm{NH}_{n}$ with the matrix algebra of size $n$ ! with coefficients in the ring of symmetric functions in $x_{1}, \ldots, x_{n}$.

More generally, for any permutation $s \in S_{n}$, let

$$
B_{n, s}:=\left\{x^{\beta} \mid 0 \leq b_{s(t)} \leq n-t, t=1, \ldots, n\right\} .
$$

Then $B_{n, s}$ is a homogeneous basis of graded free $\operatorname{Sym}_{n}$-module $\mathcal{P}_{n}$. Denoting by $U_{n, s}=$ $\mathbb{k}\left\langle B_{n, s}\right\rangle$ the $\mathbb{k}$-vector subspace of $\mathcal{P}_{n}$ with basis $B_{n}$, there is a natural isomorphism of $\mathrm{Sym}_{n}$-modules

$$
U_{n, s} \otimes_{\mathbb{k}} \operatorname{Sym}_{n} \stackrel{\cong}{\longrightarrow} \mathcal{P}_{n}
$$


taking $x^{\beta} \otimes f$ to $f x^{\beta}$ and giving an isomorphism

$$
\mathrm{NH}_{n} \cong \operatorname{END}_{\mathbb{k}}\left(U_{n, s}\right) \otimes_{\mathbb{k}} \operatorname{Sym}_{n} \cong U_{n, s} \otimes U_{n, s}^{*} \otimes \operatorname{Sym}_{n} .
$$

If $s=(1) \in S_{n}$ is the identity element, we also write $B_{n}^{+}$instead of $B_{n}=B_{n,(1)}$ and $U_{n}^{+}$instead of $U_{n}=U_{n,(1)}$. When $s$ is the maximal length permutation $w_{0}=$ $(1, n)(2, n-1) \cdots$, we denote $B_{n, w_{0}}$ and $U_{n, w_{0}}$ by $B_{n}^{-}$and $U_{n}^{-}$, correspondingly.

Let $\bar{U}_{n}=\mathcal{P}_{n} / \mathcal{P}_{n} \cdot \mathrm{Sym}_{n}^{\prime}$, where $\mathrm{Sym}_{n}^{\prime}$ is the codimension one ideal of $\mathrm{Sym}_{n}$ consisting of symmetric functions with zero constant term. Isomorphisms (40) and (44), upon modding out by $\mathrm{Sym}_{n}^{\prime}$, induce natural isomorphisms of graded $\mathbb{k}$-vector spaces

$$
U_{n} \cong \bar{U}_{n}, \quad U_{n, s} \cong \bar{U}_{n}
$$

Any $\operatorname{Sym}_{n}$-linear endomorphism of $\mathcal{P}_{n}$ preserves the subspace $\mathcal{P}_{n} \cdot \mathrm{Sym}_{n}^{\prime}$. Consequently, an element of $\mathrm{NH}_{n}$ induces an endomorphism of $\bar{U}_{n}$, and we get a surjective homomorphism

$$
\mathrm{NH}_{n}=\operatorname{END}_{\mathrm{Sym}_{n}}\left(\mathcal{P}_{n}\right) \longrightarrow \operatorname{END}_{\mathbb{k}}\left(\mathcal{P}_{n} /\left(\mathcal{P}_{n} \cdot \operatorname{Sym}_{n}^{\prime}\right)\right)=\operatorname{END}_{\mathbb{k}}\left(\bar{U}_{n}\right) \cong \mathrm{NH}_{n} / \mathrm{NH}_{n} \cdot \mathrm{Sym}_{n}^{\prime} .
$$

Denote this homomorphism

$$
\pi_{n}: \mathrm{NH}_{n} \longrightarrow \mathrm{END}_{\mathbb{k}}\left(\bar{U}_{n}\right) .
$$

The quotient map $\mathcal{P}_{n} \longrightarrow \bar{U}_{n}$ admits many sections $\bar{U}_{n} \longrightarrow \mathcal{P}_{n}$. Any such section determines an injective homomorphism

$$
\jmath_{n}^{\prime}: \mathrm{END}_{\mathbb{k}}\left(\bar{U}_{n}\right) \longrightarrow \mathrm{NH}_{n}
$$

such that $\pi_{n} \circ \jmath_{n}^{\prime}=\mathrm{Id}$. We now choose a particular section. The quotient map takes $U_{n} \subset \mathcal{P}_{n}$ bijectively onto $\bar{U}_{n}$, which we use to identify $U_{n}$ and $\bar{U}_{n}$, obtaining an injective homomorphism

$$
\jmath_{n}: \mathrm{END}_{\mathbb{k}}\left(\bar{U}_{n}\right) \longrightarrow \mathrm{NH}_{n}
$$

as the composition

$$
\begin{aligned}
& \operatorname{END}_{\mathbb{k}}\left(\bar{U}_{n}\right) \cong \operatorname{END}_{\mathbb{k}}\left(U_{n}\right) \hookrightarrow \operatorname{END}_{\mathbb{k}}\left(U_{n}\right) \otimes \operatorname{END}_{\operatorname{Sym}_{n}}\left(\operatorname{Sym}_{n}\right) \cong \\
& \operatorname{END}_{\operatorname{Sym}_{n}}\left(U_{n} \otimes \operatorname{Sym}_{n}\right) \cong \operatorname{END}_{\operatorname{Sym}_{n}}\left(\mathcal{P}_{n}\right)=\operatorname{NH}_{n} .
\end{aligned}
$$

We have $\pi_{n} \circ \jmath_{n}=$ Id. Likewise, for any permutation $s \in S_{n}$, the quotient map $\mathcal{P}_{n} \longrightarrow \bar{U}_{n}$ induces an isomorphism between vector spaces $U_{n, s}$ and $\bar{U}_{n}$, leading to a section $\jmath_{n, s}: \operatorname{END}_{\mathbb{k}}\left(\bar{U}_{n}\right) \longrightarrow \mathrm{NH}_{n}$ with $\pi_{n} \circ \jmath_{n, s}=\mathrm{Id}$.

Let

$$
\mathcal{P}_{n}^{\vee}=\operatorname{HOM}_{\operatorname{Sym}_{n}}\left(\mathcal{P}_{n}, \operatorname{Sym}_{n}\right)
$$


be the dual of $\mathcal{P}_{n}$ viewed as a (graded) Sym ${ }_{n}$-module. $\mathcal{P}_{n}^{\vee}$ is naturally a right graded $\mathrm{NH}_{n}$-module and a graded $\mathrm{Sym}_{n}$-module of graded dimension [n]!. Isomorphism (40) induces an isomorphism

$$
\mathcal{P}_{n}^{\vee} \cong U_{n}^{*} \otimes_{\mathbb{k}} \operatorname{Sym}_{n}
$$

of $\mathrm{Sym}_{n}$-modules. There is a canonical algebra isomorphism

$$
\phi^{\prime}: \mathcal{P}_{n} \otimes_{\mathrm{Sym}_{n}} \mathcal{P}_{n}^{\vee} \stackrel{\cong}{\longrightarrow} \mathrm{NH}_{n}
$$

coming from the definition of $\mathrm{NH}_{n}$ as the algebra of all $\mathrm{Sym}_{n}$-linear endomorphisms of $\mathcal{P}_{n}$.

Let $\delta(n)=\delta_{w_{0}}$ be the element of $\mathrm{NH}_{n}$ corresponding to the maximal length permutation in $S_{n}$.

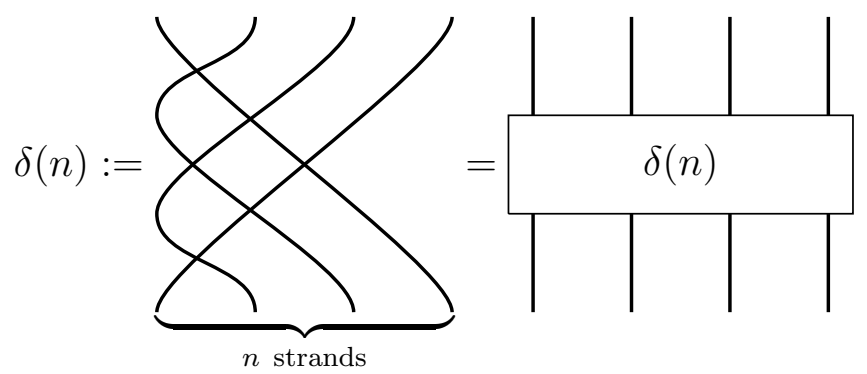

The element $\delta(n)$ spans the one-dimensional subspace of $\mathrm{NH}_{n}$ of lowest degree (degree $n(1-n))$. The operator $\delta(n)$ on $\mathcal{P}_{n}$ takes any polynomial $f$ to a symmetric polynomial.

The symmetric $\mathrm{Sym}_{n}$-bilinear pairing

$$
(-,-): \mathcal{P}_{n} \otimes_{\operatorname{Sym}_{n}} \mathcal{P}_{n} \longrightarrow \operatorname{Sym}_{n}
$$

taking $f \otimes g$ to $\delta(n)(f g)$ is nondegenerate [32, Section 2.5] and has the invariance property

$$
(y f, g)=(f, \psi(y) g), \quad y \in \mathrm{NH}_{n}, f, g \in \mathrm{Pol}_{n} .
$$

It induces an isomorphism

$$
\phi^{\circ}: \mathcal{P}_{n} \stackrel{\cong}{\longrightarrow} \mathcal{P}_{n}^{\vee}
$$

of free graded $\mathrm{Pol}_{n}$-modules taking $1 \in \mathcal{P}_{n}$ to the $\mathrm{Sym}_{n}$-linear map $1^{\vee}: \mathcal{P}_{n} \longrightarrow \operatorname{Sym}_{n}$ which sends $g \in \mathcal{P}_{n}$ to $\delta(n)(g)$. In general, under $\phi^{\circ}, f \in \mathcal{P}_{n}$ goes to the $\mathrm{Sym}_{n}$-linear map

$$
f 1^{\vee}: \mathcal{P}_{n} \longrightarrow \operatorname{Sym}_{n}, \quad g \mapsto f 1^{\vee}(g):=\delta(n)(f g) .
$$

The map $\phi^{\circ}$ can also be viewed as an isomorphism of graded right $\mathrm{NH}_{n}$-modules, where we turn $\mathcal{P}_{n}$ from a left to a right $\mathrm{NH}_{n}$-module via $\psi$.

Let $\phi_{n}=\phi^{\prime} \circ\left(\mathrm{Id} \otimes \phi^{\circ}\right)$ be the composition map

$$
\mathcal{P}_{n} \otimes_{\mathrm{Sym}_{n}} \mathcal{P}_{n} \stackrel{\operatorname{Id} \otimes \phi^{\circ}}{\longrightarrow} \mathcal{P}_{n} \otimes_{\mathrm{Sym}_{n}} \mathcal{P}_{n}^{\vee} \stackrel{\phi^{\prime}}{\longrightarrow} \mathrm{NH}_{n}
$$


This map

$$
\phi_{n}: \mathcal{P}_{n} \otimes_{\mathrm{Sym}_{n}} \mathcal{P}_{n} \longrightarrow \mathrm{NH}_{n}
$$

is an isomorphism of graded $\mathrm{NH}_{n}$-bimodules which takes $f \otimes g$ to $f \delta(n) g$.

Let $\epsilon_{n}:=(-1)^{\frac{n(n-1)}{2}} \delta(n) x_{2} x_{3}^{2} \cdots x_{n}^{n-1} \in \mathrm{NH}_{n}$. It is not hard to check that

$$
\delta(n)\left(x_{2} x_{3}^{2} \cdots x_{n}^{n-1}\right)=(-1)^{\frac{n(n-1)}{2}},
$$

so that $\epsilon_{n}(f)=f$ for any $f \in \operatorname{Sym}_{n}$. Moreover, the image of $\epsilon_{n}$ acting on $\mathcal{P}_{n}$ is exactly $\mathrm{Sym}_{n}$. This implies that $\epsilon_{n}$ is a primitive idempotent in $\mathrm{NH}_{n}$. In our graphical notation, it is depicted as follows.

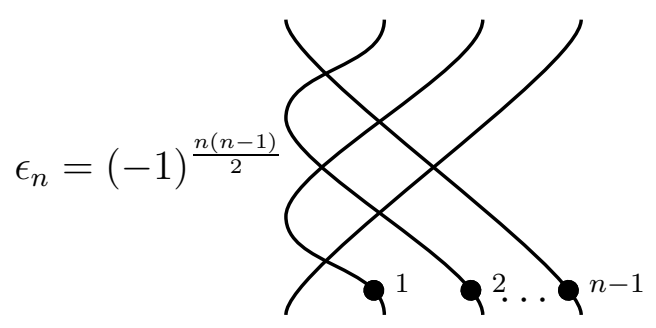

The element $\epsilon_{n}$ is homogeneous of degree 0 , and $\delta_{t} \epsilon_{n}=0$ for any $1 \leq t \leq n-1$. Up to a degree shift, the graded projective left $\mathrm{NH}_{n}$-module $\mathrm{NH}_{n} \epsilon_{n}$ is naturally isomorphic to the polynomial module $\mathcal{P}_{n}$,

$$
\mathcal{P}_{n} \cong \mathrm{NH}_{n} \epsilon_{n}\left\{\frac{n(1-n)}{2}\right\} \text {. }
$$

The isomorphism, unique up to a nonzero constant, takes the generator 1 of $\mathcal{P}_{n}$ to $\epsilon_{n}$. The modules in (58) are also isomorphic to the module induced from the onedimensional representation placed in degree $n(1-n) / 2$ of the nilCoxeter subalgebra $\mathrm{NC}_{n} \subset \mathrm{NH}_{n}$.

Consider the idempotent

$$
\epsilon_{n}^{*}=\psi \sigma\left(\epsilon_{n}\right)=x_{1}^{n-1} x_{2}^{n-2} \cdots x_{n-1} \delta(n) .
$$

Diagrammatically,

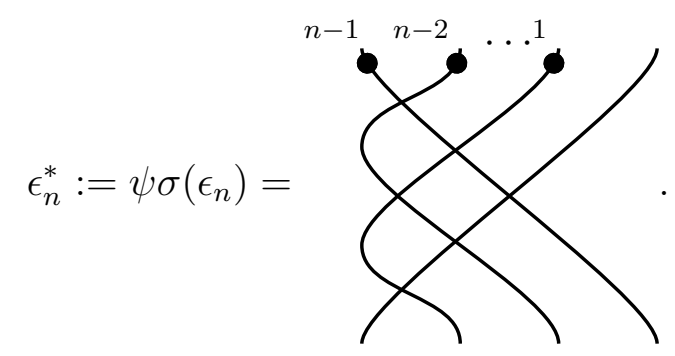

The projective right $\mathrm{NH}_{n}$-module $\epsilon_{n}^{*} \mathrm{NH}_{n}$ generated by this idempotent is isomorphic, up to grading shift, to $\mathcal{P}_{n}^{\vee}$,

$$
\epsilon_{n}^{*} \mathrm{NH}_{n}\left\{\frac{n(1-n)}{2}\right\} \cong \mathcal{P}_{n}^{\vee}
$$


via the isomorphism which takes $\epsilon_{n}^{*}$ to the functional $f \longmapsto \delta(n)(f), f \in \mathcal{P}_{n}$. The left regular representation of $\mathrm{NH}_{n}$ decomposes into $n$ ! copies of the polynomial representation $\mathcal{P}_{n}$ with grading shifts, while the right regular representation decomposes into the same number of copies of $\mathcal{P}_{n}^{\vee}$.

We recall an explicit basis of $\mathrm{NH}_{n}$ over its center $\mathrm{Sym}_{n}$ as given in [22, Section 2.5]. Denote the set of sequences $\left\{\alpha=\left(\alpha_{1}, \ldots, \alpha_{n-1}\right) \mid 0 \leq \alpha_{t} \leq t, t=1, \cdots, n-1\right\}$ by $\operatorname{Sq}(n)$. For any $\alpha \in \operatorname{Sq}(n)$, set $|\alpha|=\sum_{t=1}^{n-1} \alpha_{t}$ and $\hat{\alpha}=\left(\hat{\alpha}_{1}, \hat{\alpha}_{2}, \ldots, \hat{\alpha}_{n-1}\right):=$ $\left(1-\alpha_{1}, 2-\alpha_{2}, \ldots, n-1-\alpha_{n-1}\right)$. Define for each $\alpha \in \operatorname{Sq}(n)$,

$$
e_{\alpha}:=e_{\alpha_{1}}^{(1)} e_{\alpha_{2}}^{(2)} \cdots e_{\alpha_{n-1}}^{(n-1)}, \quad x^{\hat{\alpha}}:=x_{2}^{\hat{\alpha}_{1}} x_{3}^{\hat{\alpha}_{2}} \cdots x_{n}^{\hat{\alpha}_{n-1}} .
$$

See the beginning of Section 3.1 for the notation of symmetric functions adopted here.

Proposition 3.3. $\mathrm{NH}_{n}$ is isomorphic to the $n ! \times n$ ! matrix algebra $\mathrm{M}\left(n !, \mathrm{Sym}_{n}\right)$ over its center $\mathrm{Sym}_{n}$. A particular homogeneous matrix basis of $\mathrm{NH}_{n}$ can be given by

$$
\left\{E_{\alpha, \beta}=(-1)^{|\hat{\beta}|} e_{\alpha} \delta(n) x^{\hat{\beta}} \mid \alpha, \beta \in \operatorname{Seq}(n)\right\} .
$$

Proof. This is true because $\left\{e_{\alpha}\right\},\left\{x^{\hat{\alpha}}\right\}$ for $\alpha \in \mathrm{Sq}(n)$ form dual bases under the $\mathrm{Sym}_{n^{-}}$ bilinear pairing $(-,-)$. See [22, Proposition 2.16].

This basis will be depicted diagrammatically as follows. Abbreviating the polynomials $e_{\alpha}, x^{\hat{\beta}}$ by coupons labeled by the same symbols, the basis elements look like

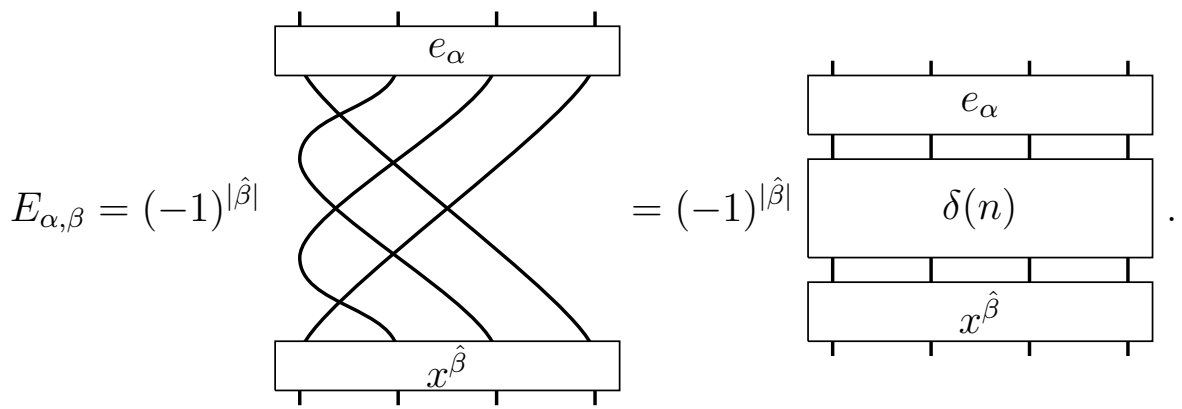

Example 3.4. $\mathrm{NH}_{2}$ provides the simplest non-trivial example for the above proposition, with basis elements given by the four diagrams below.

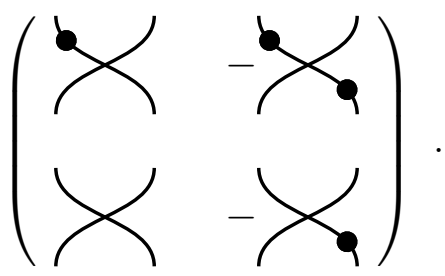


Local $p$-differentials on nilHecke algebras. We now look for $p$-differentials on nilHecke algebras compatible with the differential $\partial$ on the subalgebra $\mathrm{Pol}_{n}$ and local, in the sense of being compatible with inclusions $\mathrm{NH}_{n} \subset \mathrm{NH}_{k_{1}+n+k_{2}}$, where we add $k_{1}$, respectively $k_{2}$, vertical strands on the sides of a diagram in $\mathrm{NH}_{n}$. The nilHecke algebra is the $\mathrm{Sym}_{n}$-endomorphism algebra of the module $\mathcal{P}_{n}$, and $\mathcal{P}_{n}$ has a family of $p$-differentials $\partial_{\alpha}$ parametrized by $g_{\alpha}=\sum \alpha_{i} x_{i}, \alpha_{i} \in \mathbb{F}_{p}$, see (32). The corresponding $p$-DG $\mathrm{Pol}_{n}$-module was denoted $\mathcal{P}_{n}(\alpha)$, where $\alpha=\left(\alpha_{1}, \ldots, \alpha_{n}\right)$.

Before we move on to study $\left(\mathrm{NH}_{n}, \partial_{a}\right)$ as a $p$-DG algebra, we investigate how the differential structure of the polynomial module changes under the duality $\mathcal{P}_{n}(\alpha)^{\vee}$. Recall that $\partial_{\alpha}$ on $\mathcal{P}_{n}(\alpha)$ induces on $\mathcal{P}_{n}(\alpha)^{\vee}$ the differential $\partial_{\alpha}^{\vee}$ such that, for any $z \in \mathcal{P}_{n}(\alpha)^{\vee}$ and $f \in \mathcal{P}_{n}(\alpha)$,

$$
\partial_{\alpha}^{\vee}(z)(f)=\partial(z(f))-z\left(\partial_{\alpha}(f)\right)
$$

where the undecorated operator $\partial$ is the natural differential on the ring of symmetric polynomials (Lemma 3.1$)$. In this way $\mathcal{P}_{n}(\alpha)^{\vee}$ is naturally a $p$-DG $\left(\operatorname{Sym}_{n}, \operatorname{Pol}_{n}\right)$ bimodule since $\mathcal{P}_{n}(\alpha)$ is a $p$-DG bimodule over $\left(\mathrm{Pol}_{n}, \mathrm{Sym}_{n}\right)$. Recall from equation (55) that, under $\phi^{\circ}$, the generator $1_{\alpha} \in \mathcal{P}_{n}(\alpha)$ is sent to the Sym $n^{-l i n e a r}$ functional $1_{\alpha}^{\vee}$ which acts on any element $f$ of $\mathcal{P}_{n}(\alpha)$ as

$$
1_{\alpha}^{\vee}(f)=\phi^{\circ}\left(1_{\alpha}\right)(f)=\delta(n)(f) .
$$

In what follows, for any $\alpha=\left(\alpha_{1}, \ldots, \alpha_{n}\right) \in \mathbb{k}^{n}$, we let $\alpha^{\vee}:=\left(\alpha_{1}^{\vee}, \ldots, \alpha_{n}^{\vee}\right) \in \mathbb{k}^{n}$ and $\alpha_{t}^{\vee}:=1-n-\alpha_{t}$ for any $1 \leq t \leq n$.

Lemma 3.5. The differential $\partial_{\alpha}^{\vee}$ acts on the generator $1_{\alpha}^{\vee}$ by

$$
\partial_{\alpha}^{\vee}\left(1_{\alpha}^{\vee}\right)=\sum_{t=1}^{n} \alpha_{t}^{\vee} x_{t} 1_{\alpha}^{\vee}
$$

Equivalently, the dual polynomial module $\mathcal{P}_{n}(\alpha)^{\vee}$ with the induced differential $\partial_{\alpha}^{\vee}$ is isomorphic to $\mathcal{P}_{n}\left(\alpha^{\vee}\right)$ with the differential $\partial_{\alpha} \vee$ under the homomorphism $\phi^{\circ}$.

Proof. Since both sides of equation (62) are $\mathrm{Sym}_{n}$-linear, it suffices to check this formula on a set of $\operatorname{Sym}_{n}$-basis elements for $\mathcal{P}_{n}(\alpha)$, for instance, the set $B_{n}$ from (39)).

The left hand side of equation (62), applied to any basis element $x^{\beta}:=x_{1}^{b_{1}} \cdots x_{n}^{b_{n}}$ where $0 \leq b_{t} \leq n-t$, gives via equation (61)

$$
\partial_{\alpha}^{\vee}\left(1_{\alpha}^{\vee}\right)\left(x^{\beta}\right)=\partial\left(\delta(n)\left(x^{\beta}\right)\right)-\delta(n)\left(\partial_{\alpha}\left(x^{\beta}\right)\right)=-\sum_{t=1}^{n} \delta(n)\left(\left(b_{t}+\alpha_{t}\right) x_{1}^{b_{1}} \cdots x_{t}^{b_{t}+1} \cdots x_{n}^{b_{n}}\right) .
$$

On the other hand,

$$
\left(\sum_{t=1}^{n} \alpha_{t}^{\vee} x_{t} 1_{\alpha}^{\vee}\right)\left(x^{\beta}\right)=\sum_{t=1}^{n} \alpha_{t}^{\vee} \delta(n)\left(x_{t} \cdot x_{\beta}\right)=\sum_{t=1}^{n}\left(1-n-\alpha_{t}\right) \delta(n)\left(x_{1}^{b_{1}} \cdots x_{t}^{b_{t}+1} \cdots x_{n}^{b_{n}}\right) .
$$

Observe that $\delta(n)\left(x_{1}^{b_{1}} \cdots x_{t}^{b_{t}+1} \cdots x_{n}^{b_{n}}\right)$ can be non-zero only in the following two cases: 
(i) Each $b_{l}=n-l$ and $t=1$;

(ii) Exactly one of, say $b_{l}=n-l-1(1 \leq l \leq n-1)$, while the other $b_{l^{\prime}}=n-l^{\prime}$ for $l^{\prime} \neq l$, and either $t=l$ or $t=l+1$.

In case (i) the right hand sides of both equations are equal to $\left(1-n-\alpha_{1}\right) e_{1}$, while in case (ii) both sides are equal to $\alpha_{l+1}-\alpha_{l}$. The lemma follows.

This $p$-DG structure induces a differential on $\mathrm{NH}_{n}=\operatorname{END}_{\mathrm{Sym}_{n}}\left(\mathcal{P}_{n}(\alpha)\right)$, via

$$
\partial_{\alpha}(\xi)(f):=\partial(\xi(f))-\xi(\partial(f))
$$

for $\xi \in \operatorname{END}_{\operatorname{Sym}_{n}}\left(\mathcal{P}_{n}(\alpha)\right)$ and $f \in \mathcal{P}_{n}(\alpha)$.

Let us compute this differential on generators of $\mathrm{NH}_{n}$. On the subalgebra $\mathrm{Pol}_{n}$ the differential will restrict to the original differential $\partial$, with $\partial\left(x_{i}\right)=x_{i}^{2}$.

Due to the local nature of generators $\delta_{i}$, to compute $\partial_{\alpha}\left(\delta_{i}\right)$ we can reduce to $n=2$ case. Then $g=\alpha_{1} x_{1}+\alpha_{2} x_{2}$, and a short computation yields

$$
\partial_{\alpha}\left(\delta_{1}\right)=a_{1}-\left(a_{1}+1\right) x_{1} \delta_{1}+\left(a_{1}-1\right) x_{2} \delta_{1},
$$

where $a_{1}=\alpha_{2}-\alpha_{1}$. In general,

$$
\partial_{\alpha}\left(\delta_{i}\right)=a_{i} \operatorname{Id}-\left(a_{i}+1\right) x_{i} \delta_{i}+\left(a_{i}-1\right) x_{i+1} \delta_{i},
$$

where $a_{i}=\alpha_{i+1}-\alpha_{i}$. We would like $\partial_{\alpha}$ to be local, in the sense that the coefficients for its action on generators should not depend on $i$. This is equivalent to $a_{1}=a_{2}=\cdots=a_{n-1}$. Let $a=a_{1}$. Then $\alpha_{2}=\alpha_{1}+a$ and, in general, $\alpha_{k+1}=\alpha_{1}+k a$ and

$$
g=g\left(\alpha_{1}, a\right)=\alpha_{1} x_{1}+\left(\alpha_{1}+a\right) x_{2}+\cdots+\left(\alpha_{1}+(n-1) a\right) x_{n}
$$

We denote the corresponding $p$-DG module structure on $\mathcal{P}_{n}$ by $\mathcal{P}_{n}\left(\alpha_{1}, a\right)$, previously denoted $\mathcal{P}_{n}(\alpha)$ for $\alpha=\left(\alpha_{1}, \alpha_{1}+a, \ldots, \alpha_{1}+(n-1) a\right)$. The induced differential on $\mathrm{NH}_{n}$ depends only on $a \in \mathbb{F}_{p}$, not on $\alpha_{1}$, and will be denoted $\partial_{a}$. It is given on generators by

$$
\begin{aligned}
& \partial_{a}\left(x_{i}\right)=x_{i}^{2} \\
& \partial_{a}\left(\delta_{i}\right)=a-(a+1) x_{i} \delta_{i}+(a-1) x_{i+1} \delta_{i} .
\end{aligned}
$$

Diagrammatically,

$$
\partial_{a}\left(\partial_{a}(\boldsymbol{\phi})=\boldsymbol{\phi}=\boldsymbol{\phi}^{2}\right.
$$

Thus, the $p$-DG $\operatorname{Pol}_{n}$-module $\mathcal{P}_{n}\left(\alpha_{1}, a\right)$ for $\alpha_{1}, a \in \mathbb{F}_{p}$ induces a local differential $\partial_{a}$ on $\mathrm{NH}_{n}$, turning it into a $p$-DG algebra. The differential extends to the entire $\mathrm{NH}_{n}$ 
via the Leibniz rule $\partial(x y)=\partial(x) y+x \partial(y)$. Clearly, $\partial_{a}^{p}=0$, since this is true for the differential $\partial_{\alpha}$ on $\mathcal{P}_{n}\left(\alpha_{1}, a\right)$.

The differential $\partial_{a}$ takes a "one-strand" generator $x_{i}$ to a one-strand diagram $x_{i}^{2}$ and a "two-strand" generator $\delta_{i}$ to a linear combination of two-strand diagrams. It commutes with the obvious inclusions $\mathrm{NH}_{n} \subset \mathrm{NH}_{k_{1}+n+k_{2}}$ given by adding $k_{1}$ vertical lines to the left and $k_{2}$ vertical lines to the right of a diagram in $\mathrm{NH}_{n}$. We say that $\partial_{a}$ is a local differential on the family of algebras $\mathrm{NH}_{n}$. Notice that the defining equations (65), (66) make sense for any $a \in \mathbb{k}$.

Lemma 3.6. Under any parameter $a \in \mathbb{k}$, the equation $\partial_{a}^{p}=0$ holds on $\mathrm{NH}_{n}$ if and only if $a \in \mathbb{F}_{p}$.

Proof. The "if" part follows at once from the corresponding property $\left(\partial_{g}^{p}=0\right)$ of the differential $\partial_{g\left(\alpha_{1}, a\right)}$ on $\mathcal{P}_{n}$. Clearly, $\partial_{a}^{p}\left(x_{i}\right)=0$ for any $a \in \mathbb{k}$. It suffices to show that $\partial_{a}^{p}\left(\delta_{1}\right)=0$ precisely when $a \in \mathbb{F}_{p}$.

By a direct computation we have

$$
\begin{aligned}
\partial_{a}^{2}\left(\delta_{1}\right)= & \partial_{a}\left(a-(a+1) x_{1} \delta_{1}+(a-1) x_{2} \delta_{1}\right) \\
= & (a+1) a x_{1}^{2} \delta_{1}-2(a+1)(a-1) x_{1} x_{2} \delta_{1}+(a-1) a x_{2}^{2} \delta_{1} \\
& -(a+1) a x_{1}+a(a-1) x_{2} .
\end{aligned}
$$

Thus when $p=2, \partial_{a}^{2}\left(\delta_{1}\right)=0$ if and only if $a \in \mathbb{F}_{2}$.

Applying $\partial_{a}$ once more, we obtain

$$
\partial_{a}^{3}\left(\delta_{1}\right)=(a+1) a(a-1)\left(\left(x_{1}-x_{2}\right)^{3} \delta_{1}+\left(x_{1}-x_{2}\right)^{2}\right) .
$$

An induction shows that for any $k \geq 3$,

$$
\begin{aligned}
\partial_{a}^{k}\left(\delta_{1}\right)= & (a+1) a(a-1)\left(x_{2}-x_{1}\right)^{3} \\
& \cdot\left(\sum_{i=0}^{k-3}(-1)^{i}\left(\begin{array}{c}
k-3 \\
i
\end{array}\right) \prod_{j=0}^{k-4-i}(a+2+j) \prod_{l=0}^{i-1}(a-2-l) x_{1}^{i} x_{2}^{k-3-i} \delta_{1}\right) \\
& +(a+1) a(a-1)\left(x_{2}-x_{1}\right)^{2} \\
& \cdot\left(\sum_{i=0}^{k-3}(-1)^{i}\left(\begin{array}{c}
k-3 \\
i
\end{array}\right) \prod_{j=0}^{k-4-i}(a+2+j) \prod_{l=0}^{i-1}(a-2-l) x_{1}^{i} x_{2}^{k-3-i}\right) .
\end{aligned}
$$

The coefficient in front of each term, for a fixed $i$, is $\left(\begin{array}{c}k-3 \\ i\end{array}\right)$ times the product of sums of $a$ with some consecutive integers:

$$
(a+1) a(a-1) \prod_{j=0}^{k-4-i}(a+2+j) \prod_{l=0}^{i-1}(a-2-l)=\prod_{m=-i-1}^{k-2-i}(a+m) .
$$

When $k=p \geq 3$, the $p$ consecutive residues range over all elements of $\mathbb{F}_{p}$. It follows that

$$
(a+1) a(a-1) \prod_{j=0}^{p-2-i}(a+2+j) \prod_{l=0}^{i-1}(a-2-l)=a^{p}-a,
$$


and thus

$$
\partial_{a}^{p}\left(\delta_{1}\right)=\left(a^{p}-a\right)\left(x_{2}-x_{1}\right)^{2} \sum_{i=0}^{p-3}\left((-1)^{i}\left(\begin{array}{c}
p-3 \\
i
\end{array}\right) x_{1}^{i} x_{2}^{p-3-i}\left(\left(x_{2}-x_{1}\right) \delta_{1}+1\right)\right)
$$

which is zero if and only if $a \in \mathbb{F}_{p}$. The lemma follows.

Remark 3.7. When $p=2$, the differential $\partial_{1}$ preserves the nilCoxeter subalgebra $\mathrm{NC}_{n}$ of $\mathrm{NH}_{n}$ and acts on a crossing by

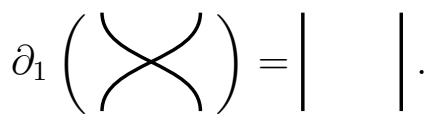

$\left(\mathrm{NC}_{n}, \partial_{1}\right)$ is a differential graded algebra, and appears in knot Floer homology [26, 27] and in categorification of quantum superalgebras [19].

Remark 3.8. Any degree -2 -nilpotent local differential $\partial^{\prime}$ acting on $\mathrm{NH}_{n}$ has the form

$$
\partial^{\prime}(\phi)=\mu \mid, \quad \partial^{\prime}\left(\gamma_{\zeta}\right)=0
$$

for some $\mu \in \mathbb{k}$. If $\mu \neq 0, \partial^{\prime}\left(\frac{x_{1}}{\mu}\right)=1$, so that $\mathrm{NH}_{n}$ is acyclic for any $n \geq 1$, and its Grothendieck group vanishes. For this reason, these differentials do not appear interesting and we will not consider them.

By Lemma 3.6, $\partial_{a}$ is a local degree two $p$-nilpotent differential on the family of algebras $\mathrm{NH}_{n}$. It is easy to check that any local degree two $p$-nilpotent differential on the family $\mathrm{NH}_{n}$ is given by $\lambda \partial_{a}$ for some $\lambda \in \mathbb{k}$ and $a \in \mathbb{F}_{p}$. Moreover, $\partial_{a}$ and $\partial_{-a}$ are related to each other by the symmetries $\psi, \sigma$ of the nilHecke algebra.

Proposition 3.9. The following equalities hold:

$$
\psi \partial_{a} \psi=\partial_{-a}, \quad \sigma \partial_{a} \sigma=\partial_{-a}
$$

Proof. The symmetry $\sigma$ of $\mathrm{NH}_{n}$ is induced by the involution on the algebra $\mathrm{Pol}_{n}$ (also denoted $\sigma$ ) transposing $x_{t}$ and $x_{n-t}, 1 \leq t \leq n-1$. This involution takes linear function $g\left(\alpha_{1}, a\right)$ to $g\left(\alpha_{1}+(n-1) a,-a\right)$, which implies the second relation in (67).

It suffices to check the first relation on generators $x_{t}$ and $\delta_{t}$, since both sides are derivations. On $x_{t}$, we have

$$
\psi \partial_{a} \psi(\boldsymbol{\phi})=\psi \partial_{a}(\boldsymbol{\phi})=\psi\left(\boldsymbol{\phi}^{2}\right)=\boldsymbol{\phi}^{2}=\partial_{-a}(\boldsymbol{\phi})
$$


while on $\delta_{t}$

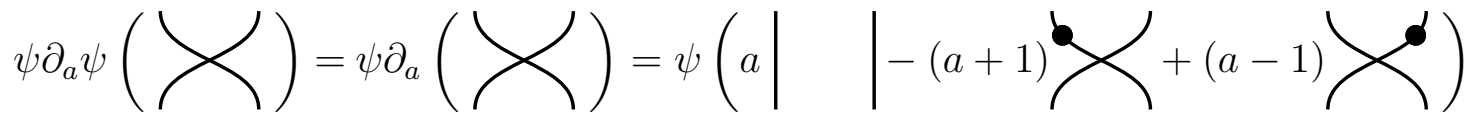

$$
\begin{aligned}
& =a|-(a+1)\rangle+(a-1)\rangle \\
& =a|\quad|-(a+1)\left(\zeta^{d}+\mid\right)+(a-1)\left(\zeta_{-} \mid\right) \\
& \left.=(-a)|\quad|-(-a+1)\rangle+(-a-1)\rangle=\partial_{-a}(\rangle\right) .
\end{aligned}
$$

The result follows.

Proposition 3.10. For any $n \in \mathbb{N}$ and $a \in \mathbb{F}_{p}$, the graded $H$-module $\mathrm{NH}_{n}$ is a compact object of $H-\underline{\bmod }$.

Proof. We must show that $\mathrm{NH}_{n}$ is quasi-isomorphic to a finite dimensional $H$-module. To do this, fix a reduced expression $s_{t_{1}} \cdots s_{t_{r}}$ for each element $w \in S_{n}$, where $s_{t}=$ $(t, t+1)$, and let $\delta_{w}:=\delta_{t_{1}} \cdots \delta_{t_{r}}$ (the element $\delta_{w}$ of $\mathrm{NH}_{n}$ depends only on $w$ and not on a reduced expression). Equation (66) and the nilHecke relations imply that

$$
\partial_{a}\left(\delta_{w}\right)=\sum_{t=1}^{n} b_{t} x_{t} \delta_{w}+\sum_{w^{\prime}} f_{w^{\prime}} \delta_{w^{\prime}}
$$

where $b_{k} \in \mathbb{F}_{p}$, permutations $w^{\prime} \in S_{n}$ appearing in the second summand have strictly fewer crossings than $w$, and $f_{w^{\prime}}$ are polynomials in $x_{i}$ 's (both $b_{k}$ and $f_{w^{\prime}}$ depend on $w$ ). The nilHecke algebra $\mathrm{NH}_{n}$ is a free left $\mathrm{Pol}_{n}$-module with a basis of elements $\delta_{w}$, over all $w \in S_{n}$. Choose a total ordering $\leq$ on $S_{n}$ which refines the partial order $w_{1}<w_{2}$ whenever $w_{1}$ has fewer crossings than $w_{2}$. Let $\tau_{1}, \tau_{2}, \ldots, \tau_{n}$ ! be the list of permutations in this order. Consider the filtration

$$
0=N_{0} \subset N_{1} \subset N_{2} \subset \cdots \subset N_{n !}=\mathrm{NH}_{n}
$$

where $N_{j}$ is the free left $\mathrm{Pol}_{n}$-submodule of $\mathrm{NH}_{n}$ spanned by $\delta_{\tau_{1}}, \delta_{\tau_{2}}, \ldots, \delta_{\tau_{j}}$.

Equations (68) show that the derivation $\partial_{a}$ preserves this filtration, $\partial_{a}\left(N_{j}\right) \subset N_{j}$, and that each subquotient $N_{j} / N_{j-1}$ is isomorphic to the p-DG $\operatorname{Pol}_{n}$-module $\mathcal{P}_{n}(\alpha)\{m\}$ for $\alpha=\left(\alpha_{1}, \ldots, \alpha_{n}\right) \in \mathbb{F}_{p}^{n}$ and some $m \in \mathbb{Z}$.

From the earlier discussion of rank one $\operatorname{Pol}_{n} p$-DG modules we know that $\mathcal{P}_{n}(\alpha)$ is quasi-isomorphic to a finite-dimensional $H$-module for any $\alpha$ (equivalently, the graded $H$-module $\mathcal{P}_{n}(\alpha)$ is isomorphic to a direct sum of a finite-dimensional $H$-module and a free $H$-module). Therefore, $\mathrm{NH}_{n}$ has the same property. 
Lemma 3.11. The differential $\partial_{0}$ acts on the element $\delta(n) \in \mathrm{NH}_{n}$ by

$$
\partial_{0}(\delta(n))=-(n-1) e_{1}^{(n)} \delta(n),
$$

which is depicted diagrammatically by

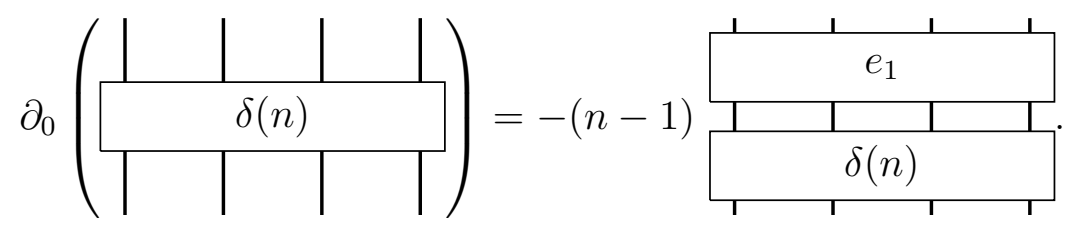

Proof. Here we give a relatively detailed proof of the formula as the same method can be used in similar situations later on. The lemma is proven by induction. The $n=1$ case is clear. Suppose we have shown the formula when $n \leq k-1$. When $n=k$, we have

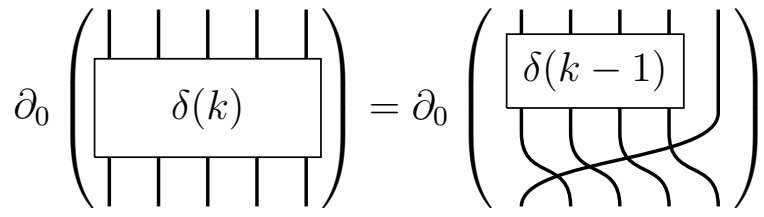

$$
\begin{aligned}
& =-(k-2) \text { 年) }
\end{aligned}
$$

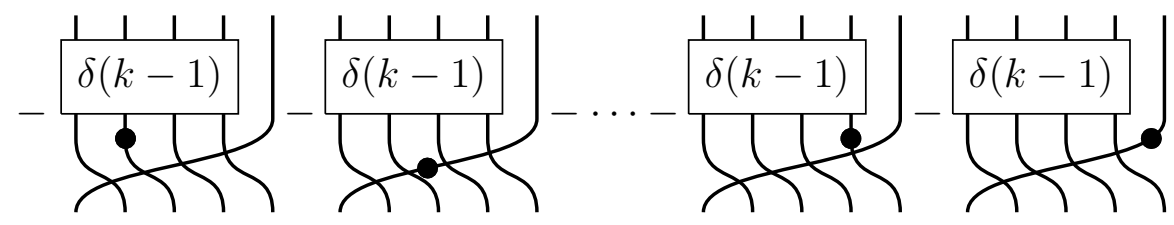

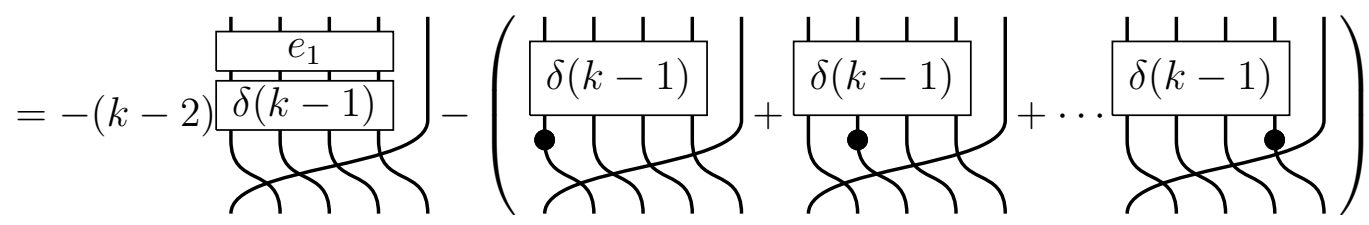

$$
\begin{aligned}
& (
\end{aligned}
$$




$$
=-(k-2) \text { (k) }
$$

where in the fourth equality, all dots on the last strand in the second parenthesized expression can slide up without obstruction essentially because $\delta_{i}^{2}=0$, while in the fifth equality we used that elements of $\operatorname{Sym}_{k-1}$ commute with $\delta(k-1)$.

Lemma 3.12. The differential $\partial_{1}$ acts on $\delta(n) \in \mathrm{NH}_{n}$ as follows:

$$
\partial_{1}(\delta(n))=-\sum_{t=1}^{n}(n-t) x_{t} \delta(n)-\sum_{t=1}^{n}(t-1) \delta(n) x_{t}
$$

which has the diagrammatic presentation

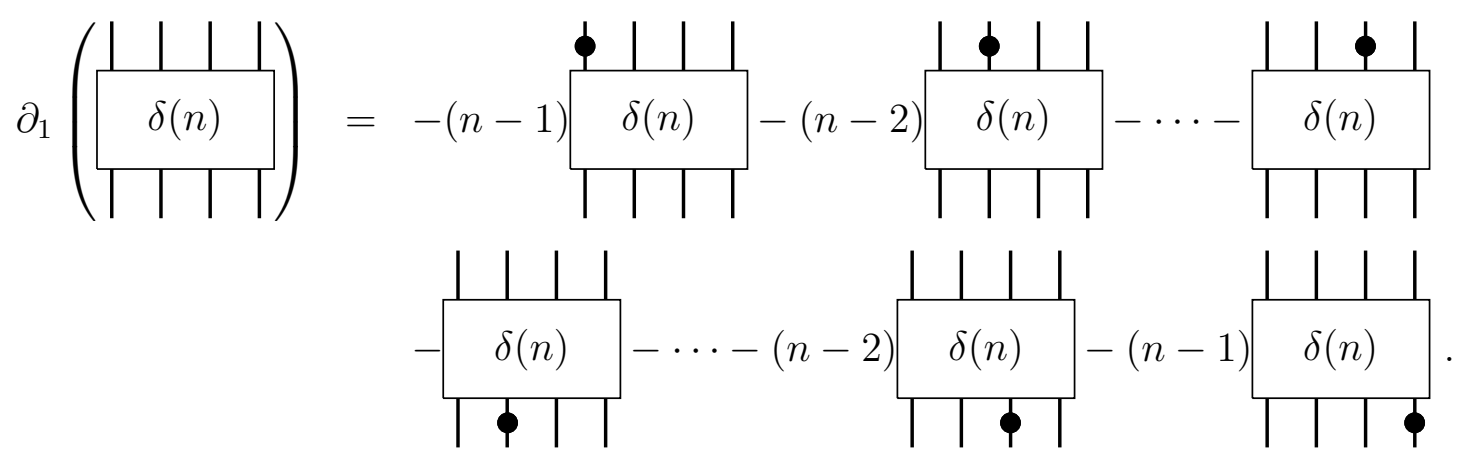

Proof. The proof is by an induction argument analogous to the one we used in the proof of the previous lemma. We leave it as an exercise.

Corollary 3.13. The differential $\partial_{a}$ acts on $\delta(n) \in \mathrm{NH}_{n}$ by

$$
\partial_{a}(\delta(n))=\left(\sum_{t=1}^{n}(t-1) a x_{t}\right) \delta(n)-\delta(n)\left(\sum_{t=1}^{n}((t-1) a+n-1) x_{t}\right) .
$$

Proof. The result follows from the previous two lemmas together with the formula $\partial_{a}=\partial_{0}+a\left(\partial_{1}-\partial_{0}\right)$.

We summarize the main results of this subsection in the following proposition.

Proposition 3.14. Let $\alpha=\left(\alpha_{1}, \ldots, \alpha_{n}\right)$ be an $n$-tuple of numbers in $\mathbb{F}_{p}$ with the property that $\alpha_{t}-\alpha_{t-1}=a$ for all $2 \leq t \leq n$. Set $\alpha^{\vee}=\left(1-n-\alpha_{1}, \ldots, 1-n-\alpha_{n}\right)$. 
(i) There is a $\partial$-invariant, non-degenerate $\mathrm{Sym}_{n}$-bilinear form

$$
(-,-): \mathcal{P}_{n}(\alpha) \otimes \mathcal{P}_{n}\left(\alpha^{\vee}\right) \longrightarrow \operatorname{Sym}_{n}, \quad f \otimes g \mapsto \delta(n)(f g),
$$

which is also compatible with the left and right $p-D G\left(\mathrm{NH}_{n}, \partial_{a}\right)$-module structures. Here being $\partial$-invariant means that $\partial(f, g)=\left(\partial_{\alpha}(f), g\right)+\left(f, \partial_{\alpha^{\vee}}(g)\right)$. The compatibility condition states that $(\xi(f), g)=(f, \psi(\xi)(g))$ and the Leibniz rule

$$
\partial(\xi(f), g)=\left(\partial_{a}(\xi)(f), g\right)+\left(\xi\left(\partial_{\alpha}(f)\right), g\right)+\left(\xi(f), \partial_{\alpha^{\vee}}(g)\right)
$$

holds for any $\xi \in \mathrm{NH}_{n}$.

(ii) There is an isomorphism $\phi^{\circ}$ of right $\left(\mathrm{NH}_{n}, \partial_{a}\right)$-modules

$$
\phi^{\circ}:\left(\mathcal{P}_{n}\left(\alpha^{\vee}\right), \partial_{\alpha^{\vee}}\right) \longrightarrow\left(\mathcal{P}_{n}(\alpha)^{\vee}, \partial_{\alpha}^{\vee}\right)
$$

where $\phi^{\circ}$ takes the generator $1_{\alpha^{\vee}} \in \mathcal{P}_{n}\left(\alpha^{\vee}\right)$ to the $\mathrm{Sym}_{n}$-linear map $1_{\alpha}^{\vee}: f \mapsto$ $\delta(n)(f)$.

(iii) There is an isomorphism of graded $p-D G\left(\mathrm{NH}_{n}, \partial_{a}\right)$-bimodules

$$
\phi_{n}: \mathcal{P}_{n}(\alpha) \otimes_{\mathrm{Sym}_{n}} \mathcal{P}_{n}\left(\alpha^{\vee}\right) \longrightarrow \mathrm{NH}_{n}, \quad f \otimes g \mapsto f \delta(n) g
$$

which equals the composition

$$
\mathcal{P}_{n}(\alpha) \otimes_{\mathrm{Sym}_{n}} \mathcal{P}_{n}\left(\alpha^{\vee}\right) \stackrel{\mathrm{Id} \otimes \phi^{\circ}}{\longrightarrow} \mathcal{P}_{n}(\alpha) \otimes_{\mathrm{Sym}_{n}} \mathcal{P}_{n}(\alpha)^{\vee} \stackrel{\phi^{\prime}}{\longrightarrow} \mathrm{NH}_{n} .
$$

Proof. Follows from Lemma 3.5. Proposition 3.9 and the explicit form of $\partial_{a}(\delta(n))$ in Corollary 3.13 .

\subsection{Specializations of $a$}

The differential $\partial_{a}$ turns $\mathrm{NH}_{n}$ into a $p$-DG algebra.

Proposition 3.15. The $p-D G$ algebra $\left(\mathrm{NH}_{n}, \partial_{a}\right)$ is acyclic for any $n \geq p$ and $a \in \mathbb{F}_{p}^{*}$.

Proof. It suffices to check the result for $n=p$. Indeed if $\mathrm{NH}_{p}$ is acyclic, by Proposition 2.21, there exists an element $y_{p}$ such that $\partial_{a}\left(y_{p}\right)=1_{\mathrm{NH}_{p}}$. Then the element $y_{p} \otimes 1_{\mathrm{NH}_{n-p}} \in$ $\mathrm{NH}_{p} \otimes \mathrm{NH}_{n-p} \subset \mathrm{NH}_{n}$ satisfies $\partial_{a}\left(y_{p} \otimes 1_{\mathrm{NH}_{n-p}}\right)=1_{\mathrm{NH}_{n}}$, and $\mathrm{NH}_{n}$ is acyclic by Proposition 2.21.

When $n=p$, pick any $\alpha_{1} \in \mathbb{F}_{p}$. The residues $\alpha_{1}, \alpha_{1}+a, \ldots, \alpha_{1}+(p-1) a$ run over all elements in $\mathbb{F}_{p}$. Let $s \in S_{p}$ be the permutation such that $s(k)=p-\alpha_{1}-(k-1) a$ modulo $p$ for $1 \leq k \leq p$. Then $\partial_{\alpha}\left(x_{1}^{s(1)} x_{2}^{s(2)} \cdots x_{p}^{s(p)}\right)=0$, where we view the argument as an element of $\mathcal{P}_{p}(\alpha)$ for $\alpha=\left(\alpha_{1}, \alpha_{1}+a, \ldots, \alpha_{1}+(p-1) a\right)$. Consequently, the subspace $U_{p, s}$ is $\partial_{\alpha}$-stable and map (44) is an isomorphism of $\left(\mathrm{Sym}_{p}, \partial_{a}\right)$-modules. 
As an $H$-module, $U_{p, s} \cong V_{0} \otimes V_{1} \otimes \cdots \otimes V_{p-1}$. The $H$-module $V_{p-1}$ is free, and $U_{p, s}$ is a free module as well. Hence, the $p$-DG algebra $\operatorname{END}_{\mathbb{k}}\left(U_{p, s}\right)$ is acyclic, and

$$
\mathrm{NH}_{p} \cong \operatorname{END}_{\operatorname{Sym}_{p}}\left(\mathcal{P}_{p}(\alpha)\right) \cong \operatorname{END}_{\operatorname{Sym}_{p}}\left(U_{p, s} \otimes \operatorname{Sym}_{p}\right) \cong \operatorname{END}_{\mathbb{k}}\left(U_{p, s}\right) \otimes \operatorname{Sym}_{p}
$$

is acyclic as well.

Example 3.16. When $\operatorname{char}(\mathbb{k})=3$, and $a=1$ one computes that

$$
\partial_{1}^{2}(\searrow \backslash)=\mid
$$

Conjugating by $\sigma$,

$$
\partial_{-1}^{2}(X X)=||
$$

This proves acyclicity of $\left(\mathrm{NH}_{3}, \partial_{ \pm 1}\right)$ via Proposition 2.21,

An argument similar to the one in Proposition 3.15] shows that, for $n=p-1$ and $a \in \mathbb{F}_{p}^{*}$, the subspace $U_{p-1, s}$ is $\partial_{\alpha}$-stable for a unique pair of $\alpha_{1}$ and $s$. As a $p$-DG algebra,

$$
\mathrm{NH}_{p-1} \cong \mathrm{END}_{\mathbb{k}}\left(U_{p-1, s}\right) \otimes \operatorname{Sym}_{p-1} .
$$

Since $\operatorname{Sym}_{p-1}$ is quasi-isomorphic to the ground field $\mathbb{k}$, the inclusion $\operatorname{END}_{\mathbb{k}}\left(U_{p-1, s}\right) \subset$ $\mathrm{NH}_{p-1}$ is a quasi-isomorphism of $p$-DG algebras. For $n<p-1$, in general, there are no permutations $s$ such that $U_{n, s}$ is $\partial_{\alpha}$-stable unless $a= \pm 1$, singling out these values of $a$, as discussed below after the proof of Proposition 3.18.

Proposition 3.15 yields the following corollary.

Corollary 3.17. For any $a \in \mathbb{F}_{p}^{*}$ and $n \geq p, \mathcal{D}\left(\mathrm{NH}_{n}, \partial_{a}\right) \cong 0$, and consequently $K_{0}\left(\mathrm{NH}_{n}, \partial_{a}\right)=0$.

Proof. Combine Proposition 3.15 with Proposition 2.21.

When $a=0$, the derived category $\mathcal{D}\left(\mathrm{NH}_{n}, \partial_{0}\right)$ does not vanish for any $n \geq 0$. This is the reason why we will disregard this case in what follows when categorifying the small quantum group $u_{\mathbb{O}_{2 p}}^{+}\left(\mathfrak{s l}_{2}\right)$.

Proposition 3.18. For any $n \in \mathbb{N}, \mathcal{D}\left(\mathrm{NH}_{n}, \partial_{0}\right) \not 0$.

Proof. By Proposition 2.21 we need to show that $\mathrm{NH}_{n}$ is not acyclic. Acyclicity of $\mathrm{NH}_{n}$ implies acyclicity of $\mathrm{NH}_{m}$ for any $m>n$. If $n=k p+1$ where $k \in \mathbb{N}$, Lemma 3.11 shows that $\partial_{0}(\delta(n))=0$. Being of the lowest degree in $\mathrm{NH}_{n}$, the element $\delta(n)$ is not in the image of $\partial_{0}$. Hence $\mathbb{k} \delta(n) \cong V_{0}$ spans a non-trivial summand in $\mathrm{H}_{/ 0}\left(\mathrm{NH}_{n}\right)$, and $\mathrm{NH}_{n}$ is not acyclic for $n=k p+1$ and, therefore, for any $n$. 
Why specialize. The main point of this subsection is that, under the specialization $a= \pm 1$, the $p$-DG algebras $\mathrm{NH}_{n}$ behave extremely well: they are quasi-isomorphic to matrix algebras (see Proposition 3.22 and Corollary 3.25), and the column modules are compact cofibrant (Proposition 3.26), which allows one to compute the Grothendieck group $K_{0}\left(\mathcal{D}\left(\mathrm{NH}_{n}, \partial_{ \pm 1}\right)\right)$, see Corollary 3.27. Another reason will become clear upon generalizing from $\mathfrak{s l}_{2}$ to arbitrary simply-laced simple Lie algebras, see Theorem 4.14 .

Recall from the beginning of Section 3.1 that the $\left(\mathrm{Pol}_{2}, \partial\right)$-module $\left(\mathcal{P}_{2}(\alpha), \partial_{\alpha}\right)$ has a generator $1_{\alpha}$ on which $\partial_{\alpha}$ acts by $\partial_{\alpha}\left(1_{\alpha}\right)=\left(\alpha_{1} x_{1}+\alpha_{2} x_{2}\right) 1_{\alpha}$, where $\alpha_{2}-\alpha_{1}=a$ is nonzero in $\mathbb{F}_{p}$. We would like to determine for which values of $a$ does there exist a two-step filtration on $\mathcal{P}_{2}(\alpha)$ whose subquotients are rank one $\left(\mathrm{Sym}_{2}, \partial\right)$-modules. This amounts to asking an equivalent question: when does $\left(\beta_{1} x_{1}+\beta_{2} x_{2}\right) 1_{\alpha}$ generate a $\left(\mathrm{Sym}_{2}, \partial\right)$-stable submodule, where $\beta_{1}, \beta_{2} \in \mathbb{k}$ are constants that are not both zero?

Proposition 3.19. The $\mathrm{Sym}_{2}$-module $\mathrm{Sym}_{2} \cdot\left(\beta_{1} x_{1}+\beta_{2} x_{2}\right) 1_{\alpha}$ is $\partial_{\alpha}$-stable only when $a= \pm 1$ (among a in $\mathbb{F}_{p}^{*}$ ). Furthermore,

- if $a=1$, then $\beta_{2}=0$ and $\beta_{1} \neq 0$;

- if $a=-1$, then $\beta_{1}=0$ and $\beta_{2} \neq 0$.

Proof. Exercise.

We see that such a filtration on $\mathcal{P}_{2}(\alpha)$ exists for $a \in \mathbb{F}_{p}^{*}$ iff $a= \pm 1$. If $a=0$, there is a unique submodule as above, generated by $\left(x_{1}-x_{2}\right) 1_{\alpha}$.

Recall that $\mathrm{NH}_{2}$ is isomorphic to the matrix algebra $\mathrm{M}\left(2, \mathrm{Sym}_{2}\right)$ (see Proposition 3.3 and Example 3.4). Such an identification is not unique. Indeed, as a $\mathrm{Sym}_{2}$-module,

$$
\mathcal{P}_{2}=\mathbb{k}\left[x_{1}, x_{2}\right] \cong \operatorname{Sym}_{2}\{2\} \oplus \operatorname{Sym}_{2} \cong \operatorname{Sym}_{2} v_{1}(b) \oplus \operatorname{Sym}_{2} v_{2},
$$

where the second summand is canonically generated by the degree zero element $v_{2}=$ $1 \in \mathrm{Pol}_{2}$, while there is a 1-parameter family of choices for the degree two generator

$$
v_{1}(b)=x_{1}+b\left(x_{1}+x_{2}\right), \quad b \in \mathbb{k} .
$$

Let us also set $v_{2}(b)=v_{2}$ for $b \in \mathbb{k}$. Under the identification $\mathrm{NH}_{2} \cong \operatorname{End}_{\mathrm{Sym}_{2}}\left(\mathcal{P}_{2}\right)$, we obtain two primitive homogeneous idempotents of $\mathrm{NH}_{2}$ depending on the parameter $b$ :

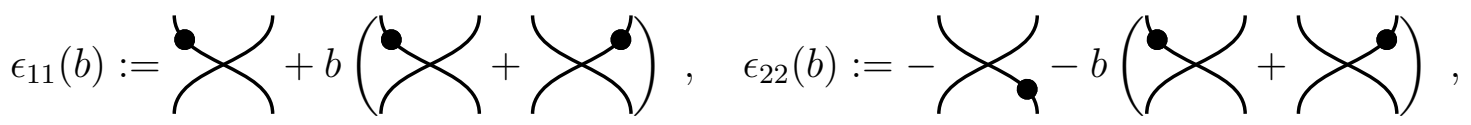

such that $\epsilon_{i i}(b) v_{j}(b)=\delta_{i j} v_{j}(b), i, j \in\{1,2\}$. Thus, $\epsilon_{11}(b)$ is a projection from $\mathrm{Pol}_{2}$ onto a summand isomorphic to $\operatorname{Sym}_{2}\{2\}$, while $\epsilon_{22}(b)$ is the projection onto the unique summand isomorphic to $\mathrm{Sym}_{2}$. When $b=0$, we recover the idempotents of Example 3.4. The elements $\epsilon_{11}(b), \epsilon_{22}(b)$ over $b \in \mathbb{k}$ are the only homogeneous minimal idempotents in $\mathrm{NH}_{2}$.

We next check for what values of $a$ there is an idempotent $\epsilon_{i i}(b)(i=1,2)$ which generates a $\partial_{a}$-stable left submodule of $\mathrm{NH}_{2}$. This property is related to the categorification of the second divided power $E^{(2)}$, see the next subsection. 
Proposition 3.20. The $\mathrm{NH}_{2}$-module $\mathrm{NH}_{2} \cdot \epsilon_{i i}(b)$ is $\partial_{a}$-stable, where $i \in\{1,2\}, b \in \mathbb{k}$ and $a \in \mathbb{F}_{p}^{*}$ if and only if either $i=2, a=1$ and $b=-1$, in which case

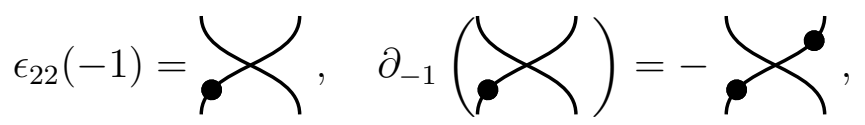

or $i=2, a=-1$ and $b=0$, in which case

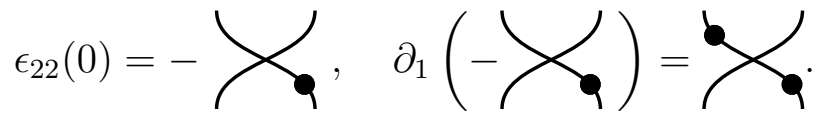

Proof. Exercise.

Notice that Propositions 3.19 and 3.20 both single out values $1,-1$ for $a \in \mathbb{F}_{p}^{*}$. The two propositions are closely related. Given a two-step $\partial_{a}$-stable filtration $0 \subset L \subset \mathcal{P}_{2}$, the left ideal of $\operatorname{END}_{\mathrm{Sym}_{2}}\left(\mathcal{P}_{2}\right)$ consisting of maps that annihilate $L$ is $\partial_{a}$-stable.

A matrix presentation. Recall that $\mathrm{Sym}_{n}^{\prime}$ is the maximal ideal of $\mathrm{Sym}_{n}$ consisting of all symmetric polynomials without the constant term. Then $\mathrm{Sym}_{n} / \mathrm{Sym}_{n}^{\prime} \cong \mathbb{k}$, and moreover $\mathrm{Sym}_{n}^{\prime} \cdot \mathrm{NH}_{n}$ is a two-sided ideal of $\mathrm{NH}_{n}$ whose quotient ring is a matrix ring:

$$
\mathrm{NH}_{n} / \operatorname{Sym}_{n}^{\prime} \cdot \mathrm{NH}_{n}=\operatorname{END}\left(\bar{U}_{n}\right) \cong \mathrm{M}(n !, \mathbb{k}) \text {. }
$$

This ideal is stable under the $\partial_{a}$ action: for any $e \in \operatorname{Sym}_{n}^{\prime}$ and $x \in \mathrm{NH}_{n}$,

$$
\partial_{a}(e \cdot x)=\partial_{a}(e) \cdot x+e \cdot \partial_{a}(x) \in \mathrm{Sym}_{n}^{\prime} \cdot \mathrm{NH}_{n},
$$

promoting the map (48) to a homomorphism of $p$-DG algebras.

Notation 3.21. In what follows, we set

$$
\left(\mathrm{M}(n !, \mathbb{k}), \partial_{a}\right):=\left(\mathrm{NH}_{n} /\left(\mathrm{Sym}_{n}^{\prime} \cdot \mathrm{NH}_{n}\right), \partial_{a}\right)
$$

to be the quotient matrix algebra over $\mathbb{k}$ with the induced differential, and denote by $\pi_{n}:\left(\mathrm{NH}_{n}, \partial_{a}\right) \longrightarrow\left(\mathrm{M}(n !, \mathbb{k}), \partial_{a}\right)$ the canonical surjective homomorphism of $p$-DG algebras.

It is obvious that $\pi_{1}: \mathrm{NH}_{1} \longrightarrow \mathrm{NH}_{1} /\left(\mathrm{Sym}_{1}^{\prime}\right)=\mathbb{k}$ and $\jmath_{1}: \mathbb{k} \hookrightarrow \mathrm{NH}_{1}$ are mutually inverse quasi-isomorphisms of $p$-DG algebras. Similar properties hold for $\mathrm{NH}_{n}(n \geq 2)$ under the $\partial_{ \pm 1}$-action. We start with the first non-trivial case. The $\partial_{1}$ action on the basis elements of $\mathrm{NH}_{2}$ from Example 3.4 is given by

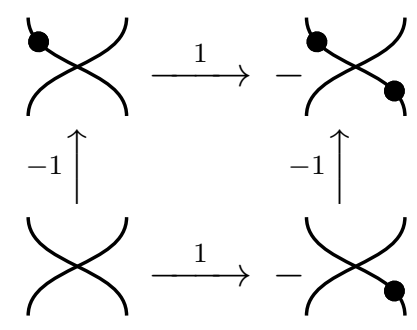


As a $\left(\mathrm{Sym}_{2}, \partial_{1}\right)$-module, the $\partial_{1}$-stable ideal $\mathrm{Sym}_{2}^{\prime} \cdot \mathrm{NH}_{2}$ admits an increasing filtration by $\left(\mathrm{Sym}_{2}, \partial_{1}\right)$-submodules:

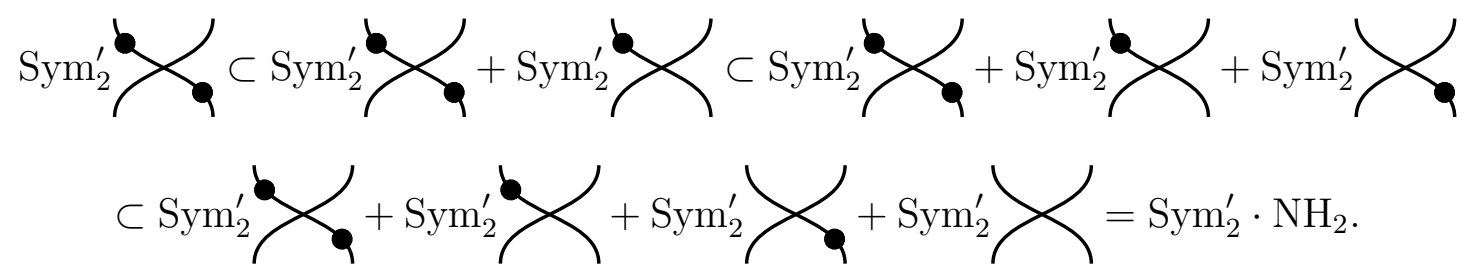

The subquotients of this filtration, as $\left(\mathrm{Sym}_{2}, \partial_{1}\right)$-modules, are all isomorphic to $\mathrm{Sym}_{2}^{\prime}$, and hence contractible. Therefore, $\mathrm{Sym}_{2}^{\prime} \cdot \mathrm{NH}_{2}$ is a contractible ideal of $\mathrm{NH}_{2}$, and $\mathrm{NH}_{2} \rightarrow \mathrm{NH}_{2} / \mathrm{Sym}_{2}^{\prime} \cdot \mathrm{NH}_{2} \cong \mathrm{M}(2, \mathbb{k})$ is a quasi-isomorphism of $p$-DG algebras. The same result holds for $\partial_{-1}$ by conjugation by $\sigma$.

Proposition 3.22. The canonical projection $\pi_{2}: \mathrm{NH}_{2} \longrightarrow \mathrm{NH}_{2} / \mathrm{Sym}_{2}^{\prime} \cdot \mathrm{NH}_{2} \cong \mathrm{M}(2, \mathbb{k})$ is a quasi-isomorphism if and only if $a= \pm 1$.

Proof. The "if" part follows from the discussion before the proposition and the symmetry between $\partial_{1}$ and $\partial_{-1}$ (Proposition 3.9). We now prove the converse. As a $\partial_{a}$-module, $\mathrm{NH}_{2}$ fits into the short exact sequence

$$
0 \longrightarrow\left\langle\boldsymbol{\phi}^{k_{1}} \boldsymbol{\phi}^{k_{2}} \mid k_{1}, k_{2} \in \mathbb{N}\right\rangle \longrightarrow \mathrm{NH}_{2} \longrightarrow\left\langle\zeta^{k_{1}} k^{k_{2}} \mid k_{1}, k_{2} \in \mathbb{N}\right\rangle \longrightarrow 0 .
$$

Here and in what follows, we use obtuse-angle brackets " \langle\rangle " to stand for the $\mathbb{k}$-linear span of the enclosed elements. The $\partial_{a}$-submodule

$$
\left\langle\boldsymbol{\phi}^{k_{1}} \boldsymbol{\phi}^{k_{2}} \mid k_{1}, k_{2} \in \mathbb{N}\right\rangle \cong \mathrm{Pol}_{2} \cong V_{0} \oplus F,
$$

decomposes into a direct sum of the trivial module $V_{0}$ and a free graded $\partial_{a}$-module $F$. On the quotient module $\partial_{a}$ acts by

$$
\left.\left.\partial_{a}\left(k_{1} \zeta^{k_{2}}\right)=\left(k_{1}-a-1\right)^{k_{1}+1} \zeta^{k_{2}}+\left(k_{2}+a-1\right)\right\rangle^{k_{1}}\right\rangle^{k_{2}+1} .
$$

Hence,

$$
\langle\overbrace{}^{k_{1}} \boldsymbol{k}^{k_{2}} \mid k_{1} \leq a+1, k_{2} \leq p+1-a\rangle
$$

is a $\partial_{a^{-}}$-submodule of the quotient, and the quotient module decomposes

$$
\left\langle\nearrow^{k_{1}}{ }^{k_{2}} \mid k_{1}, k_{2} \in \mathbb{N}\right\rangle \cong\left\langle\nearrow^{k_{1}}{ }^{k_{2}} \mid k_{1} \leq a+1, k_{2} \leq p+1-a\right\rangle \oplus F^{\prime},
$$


where $F^{\prime}$ is a free $\partial_{a}$-module. It follows that in the stable category $H$ - $\underline{\bmod }$ there is an exact triangle

$$
V_{0} \longrightarrow \mathrm{NH}_{2} \longrightarrow V_{a+1} \otimes V_{p+1-a}\{-2\} \longrightarrow V_{0}[1] .
$$

Therefore, the $\mathbb{O}_{p}$-dimension (see Notation 2.7) of $\left(\mathrm{NH}_{2}, \partial_{a}\right)$ equals

$$
\left[\mathrm{NH}_{2}\right]=1+q^{-2}\left(\sum_{i=0}^{a+1} q^{2 i}\right)\left(\sum_{j=0}^{p+1-a} q^{2 j}\right)
$$

Reduction mod $p$ gives the $\mathbb{F}_{p}$-dimension $\left[\mathrm{NH}_{2}\right]_{p}=1+(a+2)(p+2-a) \equiv 5-a^{2}$, which equals $\operatorname{dim}(\mathrm{M}(2, \mathbb{k}))=4$ modulo $p$ if and only if $a= \pm 1$. The proposition follows.

The general case of $\left(\mathrm{NH}_{n}, \partial_{ \pm 1}\right)$ for $n \geq 3$ is similar, namely the $p$-DG algebras are all quasi-isomorphic to some $(n !) \times(n !)$-matrix $p$-DG algebras. This will be shown using the $p$-DG polynomial representations of $\mathrm{NH}_{n}$. We first make some simplification of notations.

Notation 3.23. When $a=1$, let $\mathcal{P}_{n}^{+}:=\mathcal{P}_{n}\left(\alpha^{+}\right)$, where $\alpha^{+}$is the $n$-tuple $\alpha^{+}=$ $(1-n, 2-n, \ldots, 0) \in \mathbb{F}_{p}^{n}$. Likewise for $a=-1$, define $\mathcal{P}_{n}^{-}:=\mathcal{P}_{n}\left(\alpha^{-}\right)$, where $\alpha^{-}=$ $(0,-1, \cdots, 1-n)$.

Observe that $\mathcal{P}_{n}^{+}$is a left $p$-DG $\left(\mathrm{NH}_{n}, \partial_{1}\right)$-module while $\mathcal{P}_{n}^{-}$is a left module over $\left(\mathrm{NH}_{n}, \partial_{-1}\right)$. By applying $\psi$ (Proposition 3.9$), \mathcal{P}_{n}^{-}$becomes a right $p$-DG module over $\left(\mathrm{NH}_{n}, \partial_{1}\right)$. By Lemma 3.12, one can easily show that $\mathrm{NH}_{n} \epsilon_{n}$ (resp. $\left.\epsilon_{n}^{*} \mathrm{NH}_{n}\right)$ is a left (resp. right) $p$-DG ideal in $\left(\mathrm{NH}_{n}, \partial_{1}\right)$, and the isomorphism (58) (resp. (60) ) gives rise to an isomorphism of left (resp. right) $p$-DG modules:

$$
\begin{gathered}
\left(\mathcal{P}_{n}^{+}, \partial_{\alpha^{+}}\right) \cong\left(\mathrm{NH}_{n} \epsilon_{n}\left\{\frac{n(1-n)}{2}\right\}, \partial_{1}\right) \\
\left(\operatorname{resp} .\left(\mathcal{P}_{n}^{-}, \partial_{\alpha^{-}}\right) \cong\left(\epsilon_{n}^{*} \mathrm{NH}_{n}\left\{\frac{n(1-n)}{2}\right\}, \partial_{1}\right)\right) .
\end{gathered}
$$

This follows from a straightforward computation that

$$
\partial_{1}\left(\epsilon_{n}\right)=-\sum_{t=1}^{n}(n-t) x_{t} \epsilon_{n}, \quad\left(\operatorname{resp} . \partial_{1}\left(\epsilon_{n}^{*}\right)=-\sum_{t=1}^{n}(t-1) \epsilon_{n}^{*} x_{t},\right)
$$

which in turn is a direct consequence of Lemma 3.12 .

From now on till the end of this section, we will state the main results for $a= \pm 1$ while only giving the proof for $a=1$. The $a=-1$ case follows by applying the symmetry $\sigma$ (Proposition [3.9). By Proposition 3.14, there is a homogeneous $\left(\mathrm{NH}_{n}, \partial_{1}\right)$-bimodule map

$$
\phi_{n}: \mathcal{P}_{n}^{+} \otimes_{\operatorname{Sym}_{n}} \mathcal{P}_{n}^{-} \longrightarrow \mathrm{NH}_{n}
$$


which is an isomorphism. Furthermore, notice that as $p$-DG modules over $\left(\mathrm{Sym}_{n}, \partial_{1}\right)$, $\mathcal{P}_{n}^{+}$and $\mathcal{P}_{n}^{-}$have respectively as bases

$$
B_{n}^{+}:=\left\{x^{\beta}:=x_{1}^{b_{1}} x_{2}^{b_{2}} \cdots x_{n}^{b_{n}} \mid \beta=\left(b_{1}, b_{2}, \ldots, b_{n}\right), 0 \leq b_{t} \leq n-t, t=1, \ldots, n\right\},
$$

and

$$
B_{n}^{-}:=\left\{x^{\beta}:=x_{1}^{b_{1}} x_{2}^{b_{2}} \cdots x_{n}^{b_{n}} \mid \beta=\left(b_{1}, b_{2}, \ldots, b_{n}\right), 0 \leq b_{t} \leq t-1, t=1, \ldots, n\right\} .
$$

The $\mathbb{k}$-vector subspace of $\mathcal{P}_{n}^{+}$(resp. $\mathcal{P}_{n}^{-}$) spanned by $B_{n}^{+}$(resp. $B_{n}^{-}$) is stable under $\partial_{1}$. Therefore $B_{n}^{+}$(resp. $B^{-}$) is also a basis for the $\partial_{1}$-module $U_{n}^{+}=\mathbb{k}\left\langle B_{n}^{+}\right\rangle$(resp. $\left.U_{n}^{-}=\mathbb{k}\left\langle B_{n}^{-}\right\rangle\right)$. Furthermore, there are isomorphisms of $p$-DG modules over $\mathrm{Sym}_{n}$

$$
U_{n}^{+} \otimes \operatorname{Sym}_{n} \longrightarrow \mathcal{P}_{n}^{+}, \quad U_{n}^{-} \otimes \operatorname{Sym}_{n} \longrightarrow \mathcal{P}_{n}^{-},
$$

lifting the isomorphism (44). The image of the product of these basis elements under $\phi_{n}$ consists of

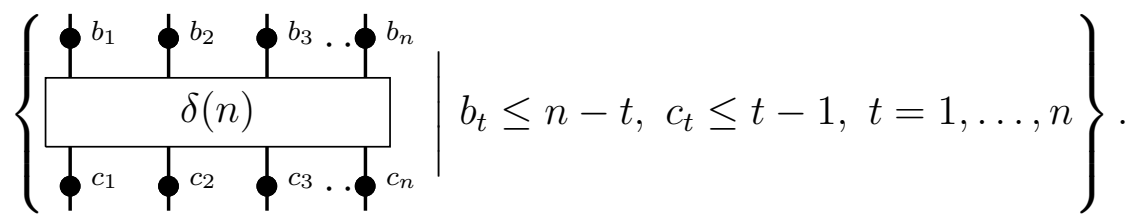

The matrix basis of $\mathrm{NH}_{n}$ given in Proposition 3.3 is obviously contained in the $\mathbb{k}$-span of the above set. Since both sets have the same cardinality $(n !)^{2}$, we conclude that they span the same subalgebra of $\mathrm{NH}_{n}$ isomorphic to the matrix algebra $\mathrm{M}(n !, \mathbb{k})$. This gives rise to a $p$-DG lifting of the homomorphism (49)

$$
\jmath_{n}:\left(\mathrm{M}(n !, \mathbb{k}), \partial_{1}\right) \hookrightarrow\left(\mathrm{NH}_{n}, \partial_{1}\right)
$$

as the composition

$$
\left(\operatorname{END}\left(U_{n}^{+}\right), \partial_{1}\right) \hookrightarrow\left(\operatorname{END}_{\mathrm{Sym}_{n}}\left(U_{n}^{+} \otimes \operatorname{Sym}_{n}\right), \partial_{1}\right) \cong\left(\operatorname{END}\left(\mathcal{P}_{n}^{+}\right), \partial_{1}\right)
$$

Similar results hold for the differential $\partial_{-1}$ as well.

Proposition 3.24. When $a= \pm 1$, the canonical surjection of $p$-DG algebras $\pi_{n}$ : $\mathrm{NH}_{n} \longrightarrow \mathrm{NH}_{n} /\left(\mathrm{Sym}_{n}^{\prime} \cdot \mathrm{NH}_{n}\right) \cong \mathrm{M}(n !, \mathbb{k})$ is a quasi-isomorphism. Furthermore, under $\partial_{1}$, a quasi-inverse of $\pi_{n}$ is given by the inclusion $\jmath_{n}: \mathrm{M}(n !, \mathbb{k}) \longrightarrow \mathrm{NH}_{n}$.

Proof. We only prove the $a=1$ case. As a $p$-DG Sym ${ }_{n}$-module, $\mathcal{P}_{n}^{+} \cong \operatorname{Sym}_{n} \otimes U_{n}^{+}$. Introduce an increasing filtration on $U_{n}^{+}$in the order of descending degrees of elements in $B_{n}^{+}$. The subquotients of this filtration on $U_{n}^{+}$are all isomorphic to degree shifted copies of $\widetilde{V}_{0}$. Therefore $\mathcal{P}_{n}^{+}$inherits a filtration whose subquotients are rank one free $p$-DG Sym $_{n}$-modules. It follows that the map of $p$-DG algebras $\pi_{n}$ factorizes as

$$
\pi_{n}:\left(\operatorname{END}\left(\mathcal{P}_{n}^{+}\right), \partial_{1}\right) \cong\left(\operatorname{END}\left(U_{n}^{+}\right) \otimes \operatorname{Sym}_{n}, \partial_{1}\right) \rightarrow\left(\operatorname{END}\left(U_{n}^{+}\right), \partial_{1}\right) \cong\left(\mathrm{M}(n !, \mathbb{k}), \partial_{1}\right)
$$


with $\jmath_{n}$ as a section. Here the middle arrow comes from the reduction of coefficient $\mathrm{Sym}_{n} \rightarrow \mathbb{k}$. Now if $n \geq p$, both sides are acyclic and the proposition is true vacuously. On the other hand, when $0 \leq n<p, \mathrm{Sym}_{n}$ is a quasi-isomorphic to $\mathbb{k}$ and the result follows.

Corollary 3.25. For any $n \in \mathbb{N}$, the functors

$\jmath_{n}^{*} \cong \pi_{n *}: \mathcal{D}\left(\mathrm{NH}_{n}, \partial_{1}\right) \longrightarrow \mathcal{D}\left(\mathrm{M}(n !, \mathbb{k}), \partial_{1}\right), \quad \pi_{n}^{*} \cong \jmath_{n_{*}}: \mathcal{D}\left(\mathrm{M}(n !, \mathbb{k}), \partial_{1}\right) \longrightarrow \mathcal{D}\left(\mathrm{NH}_{n}, \partial_{1}\right)$

are quasi-inverse equivalences of triangulated categories.

Proof. This is an easy consequence of the previous Proposition 3.24 and Theorem 2.37 ,

Under the differential $\partial_{1}$, the isomorphism $\phi_{n}$ of Proposition 3.14 restricts to an isomorphism $\left(U_{n}^{+}\right)^{*} \cong U_{n}^{-}$. Combined with the above Proposition we summarize this subsection by exhibiting the following commutative diagram

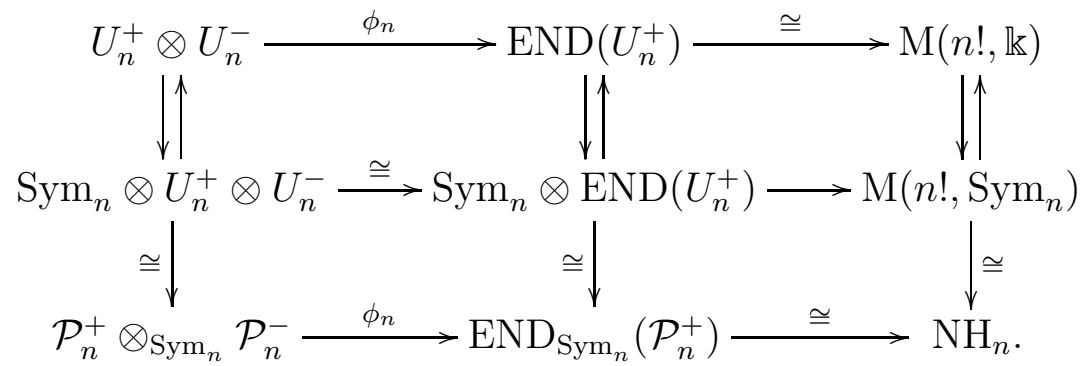

The vertical arrows in the upper half part of the diagram, given by base change with the maps of $p$-DG algebras $\mathbb{k} \hookrightarrow \mathrm{Sym}_{n}$ and $\mathrm{Sym}_{n} \rightarrow \mathbb{k}$, are all quasi-isomorphisms; while the rest of the diagram is described by Proposition 3.14 .

Compactness of the column module. Corollary 3.25 allows us to compute the Grothendieck group $K_{0}\left(\mathrm{NH}_{n}, \partial_{ \pm 1}\right)$ in the same way as for the toy model of matrix algebras (Corollary 2.45). However, we will show that the column module over $\mathrm{NH}_{n}$ is compact by exhibiting it as a direct summand of a finite cell module, which in turn leads to a more explicit classification of indecomposable modules in $\mathcal{D}^{c}\left(\mathrm{NH}_{n}, \partial_{ \pm 1}\right)$. Again, the symmetries $\psi$ and $\sigma$ allow one to focus on the differential $\partial_{1}$.

We start with the first non-trivial case $n=2$ as an example. Assume without loss of generality that $p \geq 3$. The center $\mathrm{Sym}_{2}$ of $\mathrm{NH}_{2}$ is a $p$-DG subalgebra. As a left $p$-DG module over this subalgebra, $\mathrm{NH}_{2}$ admits the following filtration by $p$-DG submodules

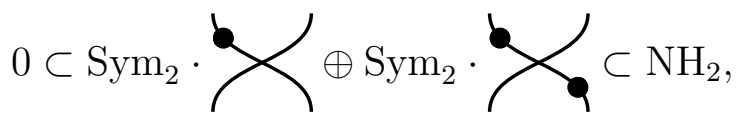


where the differential $\partial_{1}$ acts on the basis elements by

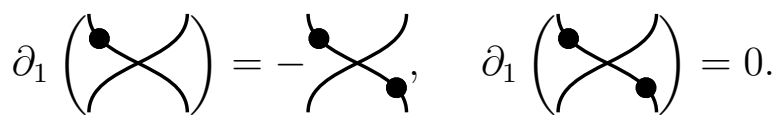

Denote the middle term by $\mathcal{P}_{2}^{-}\{1\}$. Then

$$
\mathcal{P}_{2}^{-} \cong \operatorname{Res}_{\mathrm{Sym}_{2}}^{\mathrm{NH}_{2}}\left(\mathcal{P}_{2}^{-}\right)
$$

and the filtration gives rise to a short exact sequence of $p$-DG modules over $\left(\mathrm{Sym}_{2}, \partial_{1}\right)$ :

$$
0 \longrightarrow \mathcal{P}_{2}^{-}\{1\} \longrightarrow \text { Sym }_{2} \mathrm{NH}_{2} \longrightarrow \mathcal{P}_{2}^{-}\{-1\} \longrightarrow 0 \text {. }
$$

The short exact sequence (78) comes from restricting to $\mathrm{Sym}_{2}$ the two-step filtration of $\mathrm{NH}_{2}$ as a right $p$-DG module over itself by submodules isomorphic to degree-shifts of $\mathcal{P}_{2}^{-}$. Furthermore, the left $p$-DG module ${ }_{\mathrm{Sym}_{2}} \mathrm{NH}_{2}$ over $\left(\mathrm{Sym}_{2}, \partial_{1}\right)$ decomposes into a direct sum (since $p \geq 3$ )

$$
\mathrm{Sym}_{2} \mathrm{NH}_{2} \cong \mathrm{Sym}_{2} \cdot \mid \bigoplus\left(\mathrm{Sym}_{2} \mathrm{NH}_{2}^{\prime}\right),
$$

where $\mathrm{Sym}_{2} \mathrm{NH}_{2}^{\prime}$ is the rank three $p$-DG Sym ${ }_{2}$-submodule

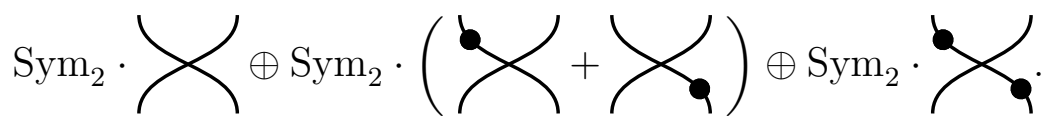

Recall that $\left(\mathrm{NH}_{2}, \partial_{1}\right)$ has the polynomial representation $\mathcal{P}_{2}^{+}$. Applying $\mathcal{P}_{2}^{+} \otimes_{\mathrm{Sym}_{2}}(-)$ to the short exact sequence (178) gives the short exact sequence of $p$-DG modules

$$
0 \longrightarrow \mathcal{P}_{2}^{+} \otimes_{\mathrm{Sym}_{2}} \mathcal{P}_{2}^{-}\{1\} \longrightarrow \mathcal{P}_{2}^{+} \otimes_{\mathrm{Sym}_{2}} \mathrm{NH}_{2} \longrightarrow \mathcal{P}_{2}^{+} \otimes_{\mathrm{Sym}_{2}} \mathcal{P}_{2}^{-}\{-1\} \longrightarrow 0
$$

We identify the terms in this short exact sequence. First off, $\mathcal{P}_{2}^{+} \otimes_{\mathrm{Sym}_{2}} \mathcal{P}_{2}^{-} \cong \mathrm{NH}_{2}$ as a left $p$-DG module over $\left(\mathrm{NH}_{2}, \partial_{1}\right)$ by Proposition 3.14. Thus the two end terms of the sequence (80) are respectively $\mathrm{NH}_{2}\{1\}$ and $\mathrm{NH}_{2}\{-1\}$. Next, the direct sum decomposition (79) gives rise to a direct sum decomposition of $\mathrm{NH}_{2}$-modules

$$
\mathcal{P}_{2}^{+} \otimes_{\mathrm{Sym}_{2}} \mathrm{NH}_{2} \cong \mathcal{P}_{2}^{+} \bigoplus\left(\mathcal{P}_{2}^{+} \otimes_{\mathrm{Sym}_{2}} \mathrm{NH}_{2}^{\prime}\right)
$$

which contains $\mathcal{P}_{2}^{+}$as a $p$-DG direct summand. Using the characterization of compact modules (Theorem 2.30), we conclude that the module $\mathcal{P}_{2}^{+}$is compact cofibrant. The general situation is proven in a similar way.

Proposition 3.26. When $0 \leq n \leq p-1$, the left $p$-DG module $\mathcal{P}_{n}^{+}$over $\left(\mathrm{NH}_{n}, \partial_{1}\right)$ is compact cofibrant. 
Proof. We claim that the module in the proposition is a direct summand of a finite cell module. The isomorphism (77) gives rise to

$$
\operatorname{Sym}_{n}\left(\mathrm{NH}_{n}\right) \stackrel{\cong}{\longrightarrow} \operatorname{Sym}_{n} \otimes U_{n}^{+} \otimes U_{n}^{-} \stackrel{\cong}{\longrightarrow} U_{n}^{+} \otimes \mathcal{P}_{n}^{-},
$$

as left $p$-DG modules over $\operatorname{Sym}_{n}$. Applying $\mathcal{P}_{n}^{+} \otimes_{\mathrm{Sym}_{n}}(-)$ to both sides, we have

$$
\mathcal{P}_{n}^{+} \otimes_{\mathrm{Sym}_{n}} \mathrm{NH}_{n} \stackrel{\cong}{\longrightarrow} U_{n}^{+} \otimes \mathcal{P}_{n}^{+} \otimes \mathcal{P}_{n}^{-} .
$$

From a finite step filtration of $U_{n}^{+}$whose associated graded modules are isomorphic to degree-shifted copies of $V_{0}$, the term on the right hand side inherits a finite step filtration whose subquotients are isomorphic to $\mathcal{P}_{n}^{+} \otimes V_{0}\{r\} \otimes \mathcal{P}_{n}^{-} \cong \mathrm{NH}_{n}\{r\}$ by Proposition 3.14. Hence the right hand side is a finite cell module. On the other hand, when $0 \leq n \leq p-1$, $n$ ! is coprime to $p$ and $\mathrm{NH}_{n}$ splits as a direct sum of $p$-DG $\left(\mathrm{Sym}_{n}, \partial_{1}\right)$-modules:

$$
\operatorname{Sym}_{n} \mathrm{NH}_{n} \cong \operatorname{Sym}_{n} \cdot I_{n !} \oplus \mathrm{NH}_{n}^{\prime},
$$

where $\mathrm{NH}_{n}^{\prime}$ denotes the space of traceless $n ! \times n !$-matrices over $\mathrm{Sym}_{n}$, which is the kernel of the canonical projection

$$
\mathrm{NH}_{n} \longrightarrow \mathrm{NH}_{n} /\left[\mathrm{NH}_{n}, \mathrm{NH}_{n}\right] \cong \mathrm{Sym}_{n} .
$$

Therefore the left hand side of (81) decomposes as

$$
\mathcal{P}_{n}^{+} \otimes_{\mathrm{Sym}_{n}} \mathrm{NH}_{n} \cong \mathcal{P}_{n}^{+} \oplus\left(\mathcal{P}_{n}^{+} \otimes_{\mathrm{Sym}_{n}} \mathrm{NH}_{n}^{\prime}\right),
$$

from which the claim follows.

Corollary 3.25]implies that $K_{0}\left(\mathrm{NH}_{n}, \partial_{1}\right) \cong K_{0}\left(\mathrm{M}(n !, \mathbb{k}), \partial_{1}\right)$. Moreover, since $\partial_{1}$ acts by taking the commutator with a fixed matrix in $\mathrm{M}(n !, \mathbb{k})$, one can directly show that $K_{0}\left(\mathrm{M}(n !, \mathbb{k}), \partial_{1}\right) \cong \mathbb{O}_{2 p}$ by the same argument as in Section 2.3. We will now give a direct proof of this using that $\mathcal{P}_{n}^{+}$is a compact generator of $\mathcal{D}\left(\mathrm{NH}_{n}, \partial_{1}\right)$, which is similar to the one used in Corollary 2.45 .

The isomorphism (77) gives rise to an isomorphism of left $p$-DG modules over $\left(\mathrm{NH}_{n}, \partial_{1}\right)$ :

$$
\mathcal{P}_{n}^{+} \otimes U_{n}^{-} \cong{ }_{\mathrm{NH}_{n}} \mathrm{NH}_{n} .
$$

Therefore, $\mathrm{NH}_{n}$ has a filtration whose subquotients are grading-shifted copies of $\mathcal{P}_{n}^{+}$. It follows that $\mathcal{P}_{n}^{+}$is a compact generator of $\mathcal{D}^{c}\left(\mathrm{NH}_{n}, \partial_{1}\right)$. Now, since $\mathrm{NH}_{n} \cong \mathrm{M}\left(n !, \operatorname{Sym}_{n}\right)$, the natural map $\operatorname{Sym}_{n} \longrightarrow \mathrm{END}_{\mathrm{NH}_{n}}\left(\mathcal{P}_{n}^{+}\right)$is an isomorphism of $p$-DG algebras.

Corollary 3.27. Let $0 \leq n \leq p-1$. Then the (underived) tensor functor

$$
\mathcal{P}_{n}^{+} \otimes_{\mathrm{Sym}_{n}}(-): \mathcal{D}\left(\mathrm{Sym}_{n}, \partial\right) \longrightarrow \mathcal{D}\left(\mathrm{NH}_{n}, \partial_{1}\right)
$$

is an equivalence of triangulated categories whose quasi-inverse is given by

$$
\mathrm{HOM}_{\mathrm{NH}_{n}}\left(\mathcal{P}_{n}^{+},-\right): \mathcal{D}\left(\mathrm{NH}_{n}, \partial_{1}\right) \longrightarrow \mathcal{D}\left(\mathrm{Sym}_{n}, \partial\right) .
$$

Consequently, the Grothendieck group $K_{0}\left(\mathrm{NH}_{n}, \partial_{ \pm 1}\right) \cong \mathbb{O}_{p}$. 
Proof. Proposition 2.34 implies that $\mathcal{P}_{n}^{+} \otimes_{\operatorname{Sym}_{n}}^{\mathbb{L}}(-)$ is an equivalence of triangulated categories. Since $\mathcal{P}_{n}^{+}$is cofibrant, the derived tensor product functor coincides with the underived one

$$
\mathcal{P}_{n}^{+} \otimes_{\operatorname{Sym}_{n}}^{\mathbb{L}}(-) \cong \mathcal{P}_{n}^{+} \otimes_{\operatorname{Sym}_{n}}(-),
$$

and likewise for the hom functor. The second claim follows from the adjunction in Proposition 2.33. Lastly, the inclusion $\mathbb{k} \longrightarrow \mathrm{Sym}_{n}$ is a quasi-isomorphism of $p$-DG algebras when $0 \leq n \leq p-1$. The result follows from Corollary 2.38,

The proof of Proposition 3.26 together with Corollary 3.27 actually gives us more information about the idempotent complete triangulated category $\mathcal{D}^{c}\left(\mathrm{NH}_{n}, \partial_{ \pm 1}\right)$. For instance, the free module $\mathrm{NH}_{n}$ fits into a convolution diagram built out of shifted copies of the polynomial representation $\mathcal{P}_{n}^{+}$, similar to the toy model case (see Remark 2.44). Modules of the form $\mathcal{P}_{n}^{+} \otimes \tilde{V}_{i}$ for all $0 \leq i \leq p-2$ and their grading shifts form a complete list of isomorphism classes of indecomposables in $\mathcal{D}^{c}\left(\mathrm{NH}_{n}, \partial_{1}\right)$.

\subsection{Grothendieck ring as $u_{\mathbb{O}_{p}}^{+}\left(\mathfrak{s l}_{2}\right)$}

The small quantum group $u_{\zeta_{2 l}}^{+}\left(\mathfrak{s l}_{2}\right)$. Let $l \geq 1$ be 2 or an odd integer. Let $\zeta_{2 l}$ be a fixed primitive $2 l$-th root of unity in $\mathbb{C}$. Then $\zeta_{l}:=\zeta_{2 l}^{2}$ is a primitive $l$-th root of unity. Following Lusztig [28, Section 5], [29, Chapter 36] (see also [5, Section V]), we define the small quantum group $u_{\zeta_{2 l}}^{+}\left(\mathfrak{s l}_{2}\right)\left(u_{\zeta_{2 l}}^{+}\right.$for short) to be the $\mathbb{Q}\left[\zeta_{2 l}\right]$-algebra with one generator $E$ subject to the relation $E^{l}=0$. Equipped with the comultiplication

$$
\Delta(E)=E \otimes 1+1 \otimes E,
$$

$u_{\zeta_{2 l}}^{+}$becomes a twisted bialgebra in the category of $\mathbb{Z} / l$-graded vector spaces. Here being twisted means that the multiplication in $u_{\zeta_{2 l}}^{+} \otimes u_{\zeta_{2 l}}^{+}$is given by

$$
\begin{gathered}
\left(E^{n} \otimes 1\right)\left(1 \otimes E^{m}\right)=E^{n} \otimes E^{m} \\
\left(1 \otimes E^{n}\right)\left(E^{m} \otimes 1\right)=\zeta_{2 l}^{n m} E^{m} \otimes E^{n}
\end{gathered}
$$

For $n \leq l-1$, the $n$-th divided power of $E$ is by definition $E^{(n)}:=\frac{E^{n}}{[n] !}\left(E^{(0)}:=1\right)$, where $[n]:=\frac{\zeta_{2 l}^{n}-\zeta_{2 l}^{-n}}{\zeta_{2 l}-\zeta_{2 l}^{-1}}=\sum_{i=1}^{n} \zeta_{2 l}^{n+1-2 i}$, and $[n] !:=\prod_{i=1}^{n}[i]$. In what follows we also denote by $\left[\begin{array}{c}m \\ n\end{array}\right]$ the quantum binomial coefficient defined as $\left[\begin{array}{c}m \\ n\end{array}\right]:=\frac{[m] !}{[n] ![m-n] !}$ whenever $n \leq m, 0 \leq n, m-n \leq l-1$. Since $[m] !=0$ if $m \geq l,\left[\begin{array}{l}l \\ n\end{array}\right]=0$. The set $\left\{E^{(r)} \mid r=\right.$ $0,1, \cdots, l-1\}$ forms an integral basis of $u_{\zeta_{2 l}}^{+}$over the ring of the $2 l$-th cyclotomic integers $\mathcal{O}_{2 l}=\mathbb{Z}\left[\zeta_{2 l}\right] \cong \mathbb{Z}[q] /\left(\Psi_{2 l}(q)\right)$, with the relation

$$
E^{(n)} \cdot E^{(m)}= \begin{cases}{\left[\begin{array}{c}
n+m \\
n
\end{array}\right] E^{(n+m)}} & \text { if } n+m \leq l-1, \\
0 & \text { otherwise. }\end{cases}
$$


This integral form will be denoted by $u_{\mathcal{O}_{2 l}}^{+}$. The comultiplication acts on on the divided power elements by

$$
\Delta\left(E^{(n)}\right)=\sum_{t=0}^{n} \zeta_{2 l}^{-t(n-t)} E^{(t)} \otimes E^{(n-t)} \in u_{\mathcal{O}_{2 l}}^{+} \otimes u_{\mathcal{O}_{2 l}}^{+} .
$$

We now specialize to $l=p$. To do this we also define an $\mathbb{O}_{p}$-integral small quantum group $u_{\mathbb{O}_{p}}^{+}\left(\mathfrak{s l}_{2}\right)\left(u_{\mathbb{O}_{p}}^{+}\right.$for short). The following elementary lemma guarantees that the divided powers $E^{(n)}$ for $0 \leq n \leq p-1$ can be defined over $\mathbb{O}_{p}$.

Lemma 3.28. In the ring $\mathbb{O}_{p}=\mathbb{Z}[q] /\left(\Psi_{p}\left(q^{2}\right)\right)$, the element

$$
[n]_{\mathbb{O}}:=\frac{q^{n}-q^{-n}}{q-q^{-1}}=\sum_{t=1}^{n} q^{n+1-2 t}
$$

is a unit if $1 \leq n \leq p-1$.

Proof. It suffices to show that $\left(1-q^{2 n}\right) /\left(1-q^{2}\right)$ is invertible. Since $q^{2}$ generates multiplicatively a cyclic group of order $p, q^{2 n}$ is another generator of the group if $1 \leq n \leq p-1$. Hence $q^{2}=\left(q^{2 n}\right)^{v}$ for some $v \in \mathbb{N}$ and

$$
\frac{1-q^{2}}{1-q^{2 n}}=\frac{1-q^{2 n v}}{1-q^{2 n}} \in \mathbb{O}_{p} .
$$

The result follows.

The lemma implies that $\left[\begin{array}{c}m \\ n\end{array}\right]_{\mathbb{O}}:=\frac{[m]_{\mathbb{O}} !}{[n]_{\mathbb{O}} ![m-n]_{\mathbb{O}} !}$ is zero if $0 \leq n, m-n \leq p-1$ and $m \geq p$. Indeed by definition $\left[\begin{array}{l}m \\ n\end{array}\right]_{\mathbb{O}}[n]_{\mathbb{O}} ![m-n]_{\mathbb{O}} !=[m]_{\mathbb{\mathbb { O }}}$ ! and the last term vanishes.

The $\mathbb{O}_{p}$-integral form $u_{\mathbb{O}_{p}}^{+}$is defined similar to $u_{\mathcal{O}_{2 p}}^{+}$: one simply replaces all the quantum integers $[n]$ in the definition of $u_{\mathcal{O}_{2 p}}^{+}$by $[n]_{\mathbb{O}}$. Recall that there is a surjective map of rings

$$
\mathbb{O}_{p} \cong \mathbb{Z}[q] /\left(1+q^{2}+\cdots+q^{2(p-1)}\right) \rightarrow \mathcal{O}_{2 p}=\mathbb{Z}\left[\zeta_{2 p}\right]
$$

by sending $q$ to $\zeta_{2 p}$. Base changing by this ring map gives rise to an isomorphism of twisted bialgebras

$$
u_{\mathbb{O}_{p}}^{+} \otimes_{\mathbb{O}_{p}} \mathcal{O}_{2 p} \cong u_{\zeta_{2 p}}^{+}
$$

In the following diagram we summarize the relations between various forms of the small quantum $\mathfrak{s l}_{2}$ introduced above and their base rings:

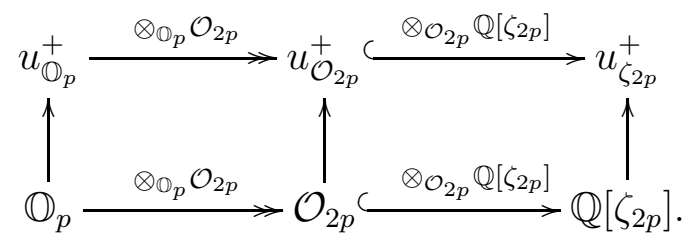

The main goal of this section is to categorify the small quantum group $u_{\mathbb{O}_{p}}^{+}$. 
Induction and restriction functors. There is an obvious inclusion of algebras

$$
\iota_{n, m}: \mathrm{NH}_{n} \otimes \mathrm{NH}_{m} \longrightarrow \mathrm{NH}_{n+m}
$$

coming from putting diagrams sideways next to each other: for any $x \in \mathrm{NH}_{n}, y \in \mathrm{NH}_{m}$,

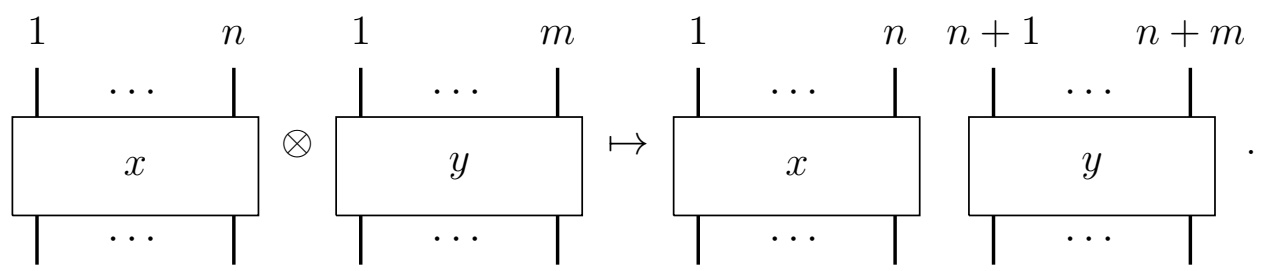

Recall from the discussion before Definition 2.32 that the tensor product $p$-DG algebra $\mathrm{NH}_{n} \otimes \mathrm{NH}_{m}$ has the differential $\partial_{a}(x \otimes y)=\partial_{a}(x) \otimes y+x \otimes \partial_{a}(y)$ for any $x \in \mathrm{NH}_{n}$ and $y \in \mathrm{NH}_{m}$. The locality of the differentials $\partial_{a}$ implies that $\iota_{n, m}$ is an inclusion of $p$-DG algebras.

The map $\iota_{n, m}$ gives rise to induction and restriction functors (Definition 2.36)

$$
\begin{aligned}
\operatorname{Ind}_{n, m} & :=\iota_{n, m}^{*}: \mathcal{D}\left(\mathrm{NH}_{n} \otimes \mathrm{NH}_{m}, \partial_{a}\right) \longrightarrow \mathcal{D}\left(\mathrm{NH}_{n+m}, \partial_{a}\right), \\
\operatorname{Res}_{n, m} & :=\iota_{n, m_{*}}: \mathcal{D}\left(\mathrm{NH}_{n+m}, \partial_{a}\right) \longrightarrow \mathcal{D}\left(\mathrm{NH}_{n} \otimes \mathrm{NH}_{m}, \partial_{a}\right) .
\end{aligned}
$$

The functor $\operatorname{Ind}_{n, m}$ preserves the subcategories of compact modules:

$$
\operatorname{Ind}_{n, m}:=\iota_{n, m}^{*}: \mathcal{D}^{c}\left(\mathrm{NH}_{n} \otimes \mathrm{NH}_{m}, \partial_{a}\right) \longrightarrow \mathcal{D}^{c}\left(\mathrm{NH}_{n+m}, \partial_{a}\right) .
$$

Likewise, there are inclusions of $p$-DG algebras under the $\partial_{a}$-action

$$
\iota_{n_{1}, n_{2}, \cdots, n_{k}}: \mathrm{NH}_{n_{1}} \otimes \mathrm{NH}_{n_{2}} \otimes \cdots \otimes \mathrm{NH}_{n_{k}} \longrightarrow \mathrm{NH}_{n_{1}+n_{2}+\cdots+n_{k}}
$$

for any sequence of $n_{i} \in \mathbb{N}$. The induction functor generalizes in an obvious way. However, it is not clear at the moment whether the restriction functor

$$
\operatorname{Res}_{n_{1}, \cdots, n_{k}}:=\iota_{n_{1}, \cdots, n_{k *}}: \mathcal{D}\left(\mathrm{NH}_{n_{1}+\cdots+n_{k}}, \partial_{a}\right) \longrightarrow \mathcal{D}\left(\mathrm{NH}_{n_{1}} \otimes \cdots \otimes \mathrm{NH}_{n_{k}}, \partial_{a}\right)
$$

preserves the subcategories of compact objects for arbitrary $a \in \mathbb{F}_{p}^{*}$.

From now on, we will focus on the special values $a= \pm 1$ while only giving the proof for the $a=1$ case. The $\partial_{-1}$ case follows by applying the symmetry $\psi$ (Proposition 3.9). Form the direct sum of $p$-DG algebras

$$
\left(\mathrm{NH}, \partial_{ \pm 1}\right):=\bigoplus_{n \in \mathbb{N}}\left(\mathrm{NH}_{n}, \partial_{ \pm 1}\right)
$$

which is no longer a unital $p$-DG algebra. The inclusion of the unital $p$-DG subalgebra $\oplus_{0 \leq n \leq p-1}\left(\mathrm{NH}_{n}, \partial_{ \pm 1}\right)$ into $\left(\mathrm{NH}, \partial_{ \pm 1}\right)$ is a quasi-isomorphism by Proposition 3.15. By 
a compact module over $\mathrm{NH}$ we mean a finite direct sum of compact modules over $\left(\mathrm{NH}_{n}, \partial_{ \pm 1}\right)$ for various $n$, so that

$$
\mathcal{D}^{c}\left(\mathrm{NH}, \partial_{ \pm 1}\right):=\bigoplus_{n \in \mathbb{N}} \mathcal{D}^{c}\left(\mathrm{NH}_{n}, \partial_{ \pm 1}\right) .
$$

On the level of Grothendieck groups, we have

$$
K_{0}\left(\mathrm{NH}, \partial_{ \pm 1}\right)=\bigoplus_{n \in \mathbb{N}} K_{0}\left(\mathrm{NH}_{n}, \partial_{ \pm 1}\right)
$$

Summing over all $n, m \in \mathbb{N}, \iota_{n, m}$ gives rise to to an induction functor between the compact derived categories

$$
\text { Ind }:=\sum_{n, m \in \mathbb{N}} \operatorname{Ind}_{n, m}: \oplus_{n, m \in \mathbb{N}} \mathcal{D}^{c}\left(\mathrm{NH}_{n} \otimes \mathrm{NH}_{m}, \partial_{ \pm 1}\right) \longrightarrow \oplus_{r \in \mathbb{N}} \mathcal{D}^{c}\left(\mathrm{NH}_{r}, \partial_{ \pm 1}\right) \text {. }
$$

Lemma 3.29. The exterior tensor product

$$
\begin{array}{ccc}
\otimes: \mathrm{NH}_{n}-\bmod \times \mathrm{NH}_{m}-\bmod & \longrightarrow & \left(\mathrm{NH}_{n} \otimes \mathrm{NH}_{m}\right)-\bmod \\
(N, M) & \mapsto & N \otimes M
\end{array}
$$

induces an isomorphism of Grothendieck groups

$$
\begin{aligned}
K_{0}\left(\mathrm{NH}_{n}, \partial_{ \pm 1}\right) \otimes_{\mathbb{O}_{p}} K_{0}\left(\mathrm{NH}_{m}, \partial_{ \pm 1}\right) & \cong K_{0}\left(\mathrm{NH}_{n} \otimes \mathrm{NH}_{m}, \partial_{ \pm 1}\right) \\
& \cong\left\{\begin{array}{cc}
\mathbb{O}_{p} & \text { if } n \leq p-1 \text { and } m \leq p-1 \\
0 & \text { otherwise. }
\end{array}\right.
\end{aligned}
$$

Proof. We show the $\partial_{1}$ case. If $n \geq p$, both $\mathrm{NH}_{n}$ and $\mathrm{NH}_{n} \otimes \mathrm{NH}_{m}$ are acyclic and the result follows, likewise for $m \geq p$. Otherwise, we use the derived equivalence of Proposition 3.24

$$
J_{n}^{*}: \mathcal{D}\left(\mathrm{NH}_{n}, \partial_{1}\right) \cong \mathcal{D}\left(\mathrm{M}(n !, \mathbb{k}), \partial_{1}\right),
$$

which takes the polynomial representation $\mathcal{P}_{n}^{+}$to the column module $\mathbb{k}^{n !}$ with the induced differential. It follows that

$$
\mathcal{D}\left(\mathrm{NH}_{n} \otimes \mathrm{NH}_{m}, \partial_{1}\right) \cong \mathcal{D}\left(\mathrm{M}(n !, \mathbb{k}) \otimes \mathrm{M}(m !, \mathbb{k}), \partial_{1}\right) \cong \mathcal{D}\left(\mathrm{M}(n ! \cdot m !, \mathbb{k}), \partial_{1}\right)
$$

and similarly for the subcategory of compact modules. Moreover, since $K_{0}\left(\mathrm{NH}_{n}, \partial_{1}\right)$ is generated as an $\mathbb{O}_{p}$-module by the symbol $\left[\mathcal{P}_{n}^{+}\right]$, using the above derived equivalence, we have

$$
K_{0}\left(\mathrm{NH}_{n} \otimes \mathrm{NH}_{m}, \partial_{1}\right) \cong K_{0}\left(\mathrm{M}(n !, \mathbb{k}) \otimes \mathrm{M}(m !, \mathbb{k}), \partial_{1}\right) \cong K_{0}\left(\mathrm{M}(n ! \cdot m !, \mathbb{k}), \partial_{1}\right),
$$

\footnotetext{
${ }^{4}$ Following Keller [17], one can say that $\left(\mathrm{NH}, \partial_{ \pm 1}\right)$ is a $p$-DG category, while objects in $\mathcal{D}^{c}\left(\mathrm{NH}, \partial_{ \pm 1}\right)$ are compact modules over this category.
} 
and, under this isomorphism, the exterior tensor product of polynomial representations is sent to

$$
\left[\mathcal{P}_{n}^{+} \otimes \mathcal{P}_{m}^{+}\right] \mapsto\left[\mathbb{k}^{n !} \otimes \mathbb{k}^{m !}\right] \mapsto\left[\mathbb{k}^{n ! \cdot m !}\right]
$$

which is the rank one free $\mathbb{O}_{p}$-module generator of $K_{0}\left(\mathrm{M}(n ! \cdot m !, \mathbb{k}), \partial_{1}\right)$. Hence $K_{0}\left(\mathrm{NH}_{n} \otimes\right.$ $\left.\mathrm{NH}_{m}, \partial_{1}\right)$ is of rank one and generated by $\left[\mathcal{P}_{n}^{+} \otimes \mathcal{P}_{m}^{+}\right]$. Since the groups $K_{0}\left(\mathrm{NH}_{n}, \partial_{1}\right)$, $K_{0}\left(\mathrm{NH}_{m}, \partial_{1}\right)$ are also of rank one, the lemma follows.

Although one can see that the restriction functor

$$
\operatorname{Res}:=\sum_{n, m \in \mathbb{N}} \operatorname{Res}_{n, m}: \oplus_{r \in \mathbb{N}} \mathcal{D}\left(\mathrm{NH}_{r}, \partial_{ \pm 1}\right) \longrightarrow \oplus_{n, m \in \mathbb{N}} \mathcal{D}\left(\mathrm{NH}_{n} \otimes \mathrm{NH}_{m}, \partial_{ \pm 1}\right)
$$

sends compact objects to compact objects, an easy computation on the Grothendieck level shows that, unlike the abelian case in [20, Proposition 2.18], the symbol of the restriction functor [Res] can not categorify the coproduct structure of $u_{\mathbb{O}_{p}}^{+}$. Indeed, by the adjunction between induction and restriction (Proposition 2.33), one has

$$
\begin{aligned}
\mathrm{NH}_{n+m} & \cong \mathbf{R H O M}_{\mathrm{NH}_{n+m}}\left(\mathrm{NH}_{n+m}, \mathrm{NH}_{n+m}\right) \\
& \cong \mathbf{R H O M}_{\mathrm{NH}_{n+m}}\left(\operatorname{Ind}_{n, m}\left(\mathrm{NH}_{n} \otimes \mathrm{NH}_{m}\right), \mathrm{NH}_{n+m}\right) \\
& \cong \mathbf{R H O M}_{\mathrm{NH}_{n} \otimes \mathrm{NH}_{m}}\left(\mathrm{NH}_{n} \otimes \mathrm{NH}_{m}, \operatorname{Res}_{n, m}\left(\mathrm{NH}_{n+m}\right)\right) .
\end{aligned}
$$

In particular, when $n+m<p$, we have the equality in the Grothendieck group

$$
([n+m] !)^{2}=\left[\mathrm{NH}_{n+m}\right]=\left[\mathbf{R H O M}_{\mathrm{NH}_{n} \otimes \mathrm{NH}_{m}}\left(\mathrm{NH}_{n} \otimes \mathrm{NH}_{m}, \operatorname{Res}_{n, m}\left(\mathrm{NH}_{n+m}\right)\right)\right],
$$

which in turn gives rise to

$$
\left[\operatorname{Res}_{n, m}\left(\mathcal{P}_{n+m}^{+}\right)\right]=\left[\begin{array}{c}
m+n \\
n
\end{array}\right]_{\mathbb{O}}\left[\mathcal{P}_{n}^{+} \otimes \mathcal{P}_{m}^{+}\right]
$$

Here we used that $\left[\mathrm{NH}_{n}\right]=\left[\mathcal{P}_{n}^{+} \otimes U_{n}^{-}\right]=[n]_{\mathbb{O}} !\left[\mathcal{P}_{n}^{+}\right]=\left([n]_{\mathbb{O}} !\right)^{2}\left[\operatorname{Sym}_{n}\right]=\left([n]_{\mathbb{O}} !\right)^{2}$ in $K_{0}(H-\underline{\bmod })=\mathbb{O}_{p}$ (see equation (77)). This mismatches with the comultiplication action given by equation (82).

A twisted restriction functor. To categorify the comultiplication of $u_{\mathbb{O}}^{+}$, we introduce a twisted version of the restriction functor.

Definition 3.30. Let $n, m \in \mathbb{N}$. We define the twisted $p$-DG bimodule ${ }_{n, m}^{X} \mathrm{NH}_{n+m}$ over $\left(\mathrm{NH}_{n} \otimes \mathrm{NH}_{m}, \mathrm{NH}_{n+m}\right)$ as follows. As an $\left(\mathrm{NH}_{n} \otimes \mathrm{NH}_{m}, \mathrm{NH}_{n+m}\right)$-bimodule, it is isomorphic to $\mathrm{NH}_{n+m}$, with the bimodule generator ${ }^{X} 1_{n+m}$ sitting in degree zero. It is twisted in the sense that the differential acts on the bimodule generator ${ }^{X} 1_{n+m} \in \underset{n, m}{X} \mathrm{NH}_{n+m}$ by

$$
\partial\left({ }^{X} 1_{n+m}\right)=m \sum_{t=1}^{n} x_{t} \cdot{ }^{X} 1_{n+m}=m e_{1}^{(n)} \cdot{ }^{X} 1_{n+m} .
$$


Remark 3.31. It is not hard to see that the twisted bimodule $\underset{n, m}{X} \mathrm{NH}_{n+m}$ is isomorphic, up to a grading shift and as a $p$-DG bimodule, to the submodule $\left(e_{n}^{(n)}\right)^{m} \cdot \mathrm{NH}_{n+m} \subset$ $\mathrm{NH}_{n+m}$, where $\left(e_{n}^{(n)}\right)^{m}=x_{1}^{m} \cdots x_{n}^{m}$, with the inherited differential. Notice that $\left(e_{n}^{(n)}\right)^{m}$ is a central element in $\mathrm{NH}_{n} \otimes \mathrm{NH}_{m}$.

More generally, if ${ }_{A} M_{B}$ is a $p$-DG bimodule over $(A, B)$ and $z$ is a central element of $A$ that is a non-zero-divisor on $M$, then ${ }^{z} M:=z \cdot M$ is again a bimodule with the same underlying $(A, B)$-bimodule structure, but with the induced differential action from the inclusion ${ }^{z} M \subset M$. We say that ${ }^{z} M$ is obtained as a $p$-DG bimodule from $M$ by twisting the differential.

For any element $y \in \mathrm{NH}_{n+m}$, we will denote by ${ }^{X} y \in \underset{n, m}{X} \mathrm{NH}_{n+m}$ the corresponding element in the twisted bimodule. Consider the element ${ }^{X} \delta(n+m) \in \underset{n, m}{X} \mathrm{NH}_{n+m}$ arising from the longest length element of $S_{n+m}$. From the differential formula on $\delta(n+m)$ (Lemma 3.12), one computes that

$$
\begin{aligned}
\partial\left({ }^{X} \delta(n+m)\right)= & -\sum_{t=1}^{n}(n-t) x_{t} \cdot{ }^{X} \delta(n+m)-\sum_{t=1}^{m}(m-t) x_{n+t} \cdot{ }^{X} \delta(n+m) \\
& -\sum_{t=1}^{n+m}(t-1)^{X} \delta(n+m) \cdot x_{t} .
\end{aligned}
$$

It follows from this equation that the right $\mathrm{Pol}_{n+m}$-span of the elements in the set $\left\{x_{1}^{b_{1}} \cdots x_{n}^{b_{n}} x_{n+1}^{c_{1}} \cdots x_{n+m}^{c_{m}} X \delta(n+m)\right\}$, where $0 \leq b_{t} \leq n-t, t=1, \ldots, n$, and $0 \leq$ $c_{v} \leq m-v, v=1, \ldots, m$, constitutes a right $p$-DG module over $\mathrm{NH}_{n+m}$ which will be denoted ${ }^{X} N_{n+m}$. Using the structure of $\mathcal{P}_{n+m}^{-}$(see Notation 2.7 and the isomorphism (77)), one readily sees that ${ }^{X} N_{n+m}$ is cofibrant as a right $p$-DG module whenever $0 \leq n+m \leq p-1$ : it has an $n$ ! $m$ !-step filtration whose subquotients are isomorphic to grading shifted copies of $\mathcal{P}_{n+m}^{-}$.

Lemma 3.32. Let $n, m$ be natural numbers with $n+m \leq p-1$.

(i) The inclusion of ${ }^{X} N_{n+m}$ into ${ }_{n, m}^{X} \mathrm{NH}_{n+m}$ is a quasi-isomorphism of right $p-D G$ modules over $\mathrm{NH}_{n+m}$.

(ii) The module $\underset{n, m}{X} \mathrm{NH}_{n+m}$ is cofibrant as a left $p-D G$ module over $\mathrm{NH}_{n} \otimes \mathrm{NH}_{m}$.

Proof. To prove the first statement, we compute the cohomology of both modules. Abbreviating the element $x_{1}^{n-1} x_{2}^{n-2} \cdots x_{n-1} x_{n+1}^{m-1} x_{n+2}^{m-2} \cdots x_{n+m-1}{ }^{X} \delta(n+m)$ by ${ }^{X} D_{n+m}$, we see from equation (88) that

$$
\partial\left({ }^{X} D_{n+m}\right)=-\sum_{t=1}^{n+m}(t-1)^{X} D_{n+m} \cdot x_{t} \in{ }^{X} D_{n+m} \cdot \mathrm{Pol}_{n+m}
$$


Then it is easy to identify the cohomology of ${ }^{X} N_{n+m}$ with the finite dimensional $\partial$-stable space

$$
\mathbb{k}\left\langle x_{1}^{b_{1}} \cdots x_{n}^{b_{n}} x_{n+1}^{c_{1}} \cdots x_{n+m}^{c_{m}}{ }^{X} \delta(n+m) x_{1}^{d_{1}} \cdots x_{n+m}^{d_{n+m}}\right\rangle,
$$

where $0 \leq b_{t} \leq n-t, t=1, \ldots, n, 0 \leq c_{t} \leq m-t, t=1, \ldots, m$, and $0 \leq d_{t} \leq t-1,1 \leq$ $t \leq n+m$. On the other hand, regarded as a left $p$-DG module over $\mathrm{NH}_{n} \otimes \mathrm{NH}_{m}$, ${ }_{n, m}{ }^{X} \mathrm{NH}_{n+m}$ has an $(n+m)$ !-step filtration whose subquotients are all isomorphic to graded shifts of the module $\operatorname{Pol}_{n+m} \cdot{ }^{X} \delta_{n+m} x_{1}^{0} x_{2}^{1} \cdots x_{n+m}^{n+m-1}$, which is isomorphic to the cofibrant $\mathrm{NH}_{n} \otimes \mathrm{NH}_{m}$ module $\mathcal{P}_{n}^{+} \otimes \mathcal{P}_{m}^{+}$. Therefore, the cohomology of $\underset{n, m}{X} \mathrm{NH}_{n+m}$ is also isomorphic to the above finite dimensional $p$-complex (89). The first result follows.

The second claim follows from the proof of the first, since as a left $p$-DG module over $\mathrm{NH}_{n} \otimes \mathrm{NH}_{m}$, the subquotients of the filtration on ${ }_{n, m}^{X} \mathrm{NH}_{n+m}$, isomorphic to $\mathcal{P}_{n}^{+} \otimes \mathcal{P}_{m}^{+}$, are cofibrant when $n+m<p$ (Proposition 3.26).

Definition 3.33. The (derived) twisted restriction ${ }^{X} \operatorname{Res}_{n, m}$ is the functor

$$
\begin{aligned}
{ }^{X} \operatorname{Res}_{n, m}: \mathcal{D}\left(\mathrm{NH}_{n+m}, \partial_{1}\right) & \longrightarrow \mathcal{D}\left(\mathrm{NH}_{n} \otimes \mathrm{NH}_{m}, \partial_{1}\right) \\
M & \mapsto \underset{n, m}{X} \mathrm{NH}_{n+m} \otimes \underset{\mathrm{NH}_{n+m}}{\mathbb{L}} M
\end{aligned}
$$

Note that by Lemma 3.32, there is a functorial isomorphism of $p$-complexes

$$
{ }^{X} \operatorname{Res}_{n, m}(M) \cong{ }^{X} N_{n+m} \otimes_{\mathrm{NH}_{n+m}} M .
$$

In what follows, we will abbreviate the $p$-DG algebra $\mathrm{NH}_{n} \otimes \mathrm{NH}_{m}$ by $\mathrm{NH}_{n, m}$, ${ }_{n, m}^{X} \mathrm{NH}_{n+m}$ by ${ }^{X} \mathrm{NH}_{n+m}$ if no confusion can be made. When we have three integers $k, n, m$ such that $0 \leq k+n+m \leq p-1$, there are two ways of composing functors, namely $\left({ }^{X} \operatorname{Res}_{k, n} \otimes \operatorname{Id}_{m}\right) \circ\left({ }^{X} \operatorname{Res}_{k+n, m}\right)$ or $\left(\operatorname{Id}_{k} \otimes{ }^{X} \operatorname{Res}_{n, m}\right) \circ\left({ }^{X} \operatorname{Res}_{k, n+m}\right)$, which are in fact both isomorphic to derived tensoring with the $\left(\mathrm{NH}_{k, n, m}, \mathrm{NH}_{k+n+m}\right)$-bimodule

$$
\left(\left(e_{k}^{(k)}\right)^{n}\left(e_{k+n}^{(k+n)}\right)^{m}\right) \cdot \mathrm{NH}_{k+n+m}=\left(x_{1} \cdots x_{k}\right)^{n+m}\left(x_{k+1} \cdots x_{k+n}\right)^{m} \cdot \mathrm{NH}_{k+n+m} .
$$

To see this one uses Lemma 3.32 to identify the composition functor, for instance, as

$$
{ }^{X} \operatorname{Res}_{k, n} \otimes \operatorname{Id}_{m}\left({ }^{X} \operatorname{Res}_{k+n, m}(M)\right) \cong\left(\left({ }^{X} \mathrm{NH}_{k+n} \otimes \mathrm{NH}_{m}\right) \otimes_{\mathrm{NH}_{k+n, m}}\left({ }^{X} \mathrm{NH}_{k+n+m}\right)\right) \otimes \mathbb{L}(M),
$$

where the cofibrance of ${ }^{X} \mathrm{NH}_{k+n+m}$ as a left $p$-DG module over $\mathrm{NH}_{k+n, m}$ allows us to replace the middle derived tensor by the usual one.

Lemma 3.34. Let $n, m$ be natural numbers with $n+m \leq p-1$. Then:

(i) the twisted restriction functor acts on the cofibrant module $\mathcal{P}_{n+m}^{+}$by

$$
{ }^{X} \operatorname{Res}_{n, m}\left(\mathcal{P}_{n+m}^{+}\right) \cong \mathcal{P}_{n}^{+} \otimes \mathcal{P}_{m}^{+}\{-m n\},
$$

(ii) the twisted restriction functor sends compact modules to compact modules. 
Proof. When acting on a cofibrant module, the twisted restriction functor need not be derived:

$$
{ }^{X} \operatorname{Res}_{n, m}\left(\mathcal{P}_{n+m}^{+}\right) \cong\left(e_{n}^{(n)}\right)^{m} \cdot \mathcal{P}_{n+m}^{+}=\left(x_{1} \cdots x_{n}\right)^{m} \cdot \mathcal{P}_{n+m}^{+} .
$$

The differential acts on the twisted module generator ${ }^{X} 1_{\alpha^{+}}$, which is identified with $\left(x_{1} \cdots x_{n}\right)^{m} \cdot 1_{\alpha^{+}}$under the above isomorphism, by

$$
\partial\left({ }^{X} 1_{\alpha^{+}}\right)=-\sum_{t=1}^{n}(n-t) x_{t} \cdot{ }^{X} 1_{\alpha^{+}}-\sum_{t=1}^{m}(m-t) x_{n+m-t} \cdot{ }^{X} 1_{\alpha^{+}} .
$$

The first statement follows by comparing with the differentials on $\mathcal{P}_{n}^{+}, \mathcal{P}_{m}^{+}$(Notation 3.23).

The second statement follows from the classification of compact modules (see the discussion after Corollary 3.27) in $\mathcal{D}\left(\mathrm{NH}_{n+m}, \partial_{ \pm 1}\right)$.

Categorification. As for the induction functor, we sum ${ }^{X} \operatorname{Res}_{n, m}$ over all $n, m \in \mathbb{N}$ and obtain the twisted restriction functor between the derived categories

$$
{ }^{X} \operatorname{Res}:=\sum_{n, m \in \mathbb{N}}{ }^{X} \operatorname{Res}_{n, m}: \oplus_{r \in \mathbb{N}} \mathcal{D}\left(\mathrm{NH}_{r}, \partial_{ \pm 1}\right) \longrightarrow \oplus_{n, m \in \mathbb{N}} \mathcal{D}\left(\mathrm{NH}_{n} \otimes \mathrm{NH}_{m}, \partial_{ \pm 1}\right) .
$$

Together with earlier discussion, Lemma 3.34 allows us to pass from the induction and twisted restriction functors on the derived category of compact NH-modules to maps of Grothendieck groups, as $\mathbb{O}_{p}$-modules,

$$
\begin{gathered}
{[\text { Ind }]: K_{0}\left(\mathrm{NH}, \partial_{ \pm 1}\right) \otimes_{\mathbb{O}_{p}} K_{0}\left(\mathrm{NH}, \partial_{ \pm 1}\right) \longrightarrow K_{0}\left(\mathrm{NH}, \partial_{ \pm 1}\right),} \\
{\left[{ }^{X} \text { Res }\right]: K_{0}\left(\mathrm{NH}, \partial_{ \pm 1}\right) \longrightarrow K_{0}\left(\mathrm{NH}, \partial_{ \pm 1}\right) \otimes_{\mathbb{O}_{p}} K_{0}\left(\mathrm{NH}, \partial_{ \pm 1}\right) .}
\end{gathered}
$$

On Grothendieck groups, it follows from the associativity of Ind that [Ind] equips $K_{0}\left(\mathrm{NH}, \partial_{ \pm 1}\right)$ with the structure of an $\mathbb{O}_{p}$-algebra whose unit is given by the symbol of $[\mathbb{k}]$ over $\left(\mathrm{NH}_{0}, \partial_{ \pm 1}\right) \cong\left(\mathbb{k}, \partial_{ \pm 1}\right)$. Likewise, $\left[{ }^{X} \mathrm{Res}\right]$ gives rise to a coassociative coalgebra structure on $K_{0}\left(\mathrm{NH}, \partial_{ \pm 1}\right)$, whose counit is given by projection onto the $p$-DG subalgebra $\mathrm{NH}_{0} \subset \mathrm{NH}$. Furthermore, a direct computation shows that

$$
\left[{ }^{X} \text { Res }\right]: K_{0}\left(\mathrm{NH}, \partial_{ \pm 1}\right) \longrightarrow K_{0}\left(\mathrm{NH}, \partial_{ \pm 1}\right) \otimes_{\mathbb{O}_{p}} K_{0}\left(\mathrm{NH}, \partial_{ \pm 1}\right)
$$

is a map of algebras, where the algebra structure on $K_{0}\left(\mathrm{NH}, \partial_{ \pm 1}\right) \otimes_{\mathbb{O}_{p}} K_{0}\left(\mathrm{NH}, \partial_{ \pm 1}\right)$ is twisted in the sense that for homogeneous elements $x_{1}, x_{2}, y_{1}, y_{2} \in K_{0}\left(\mathrm{NH}, \partial_{ \pm 1}\right)$,

$$
\left(x_{1} \otimes x_{2}\right) \cdot\left(y_{1} \otimes y_{2}\right)=q^{\left|x_{2}\right|\left|y_{1}\right|} x_{1} y_{1} \otimes x_{2} y_{2} .
$$

Theorem 3.35. There is an isomorphism of twisted bialgebras

$$
K_{0}\left(\mathrm{NH}, \partial_{ \pm 1}\right) \cong u_{\mathbb{O}_{p}}^{+}
$$

under which the symbols [Ind], $\left[{ }^{X}\right.$ Res] of the functors Ind, ${ }^{X}$ Res are identified with the multiplication and comultiplication in $u_{\mathbb{O}_{p}}^{+}\left(\mathfrak{s l}_{2}\right)$, and the symbol of the polynomial representation $\left[\mathcal{P}_{n}^{+}\right]$is identified with the $n$-th divided power $E^{(n)}$. 
Remark 3.36 (Degree one and zero differentials). Base changing $\mathbb{O}_{p}$ to $\mathcal{O}_{2 p}$ in Theorem 3.35 gives rise to an isomorphism of twisted $\mathcal{O}_{2 p}$-bialgebras

$$
K_{0}\left(\mathrm{NH}, \partial_{ \pm 1}\right) \otimes_{\mathbb{O}_{p}} \mathcal{O}_{2 p} \cong u_{\mathcal{O}_{2 p}}^{+}
$$

Here we sketch how one can categorify $u_{\mathcal{O}_{2 p}}^{+}$directly by rescaling the gradings involved.

Since the nilHecke algebra is concentrated in even degrees, one may restrict the category of modules we considered before to the full subcategory of NH-modules which are only concentrated in even degrees. Alternatively, rescale the gradings of $x_{i}$ and $\delta_{i}$ to be 1 and -1 respectively, and make the differential $\partial_{a}$ to be of degree one. All the earlier results are valid except that we do not have balanced indecomposable $H$ modules $\widetilde{V}_{k}$ any more. The stable Grothendieck ring of the Hopf algebra $H=\mathbb{k}[\partial] /\left(\partial^{p}\right)$ is isomorphic to the ring of $p$-th cyclotomic integers $\mathcal{O}_{p} \cong \mathcal{O}_{2 p}$, and the construction of this section goes through without essential change, leading to a categorification of the integral form $u_{\mathcal{O}_{2 p}}^{+}$over $\mathcal{O}_{2 p}$.

Suppose that, instead, we forget about all the gradings involved. The stable category of finite dimensional ungraded $H$-modules has its Grothendieck ring isomorphic to $\mathbb{F}_{p}$. In parallel with the above story, the ungraded compact module category over the ungraded $p$-DG algebra $\left(\mathrm{NH}, \partial_{ \pm 1}\right)$ categorifies the restricted universal enveloping algebra $u^{+}\left(\mathfrak{s l}_{2}\right) \cong \mathbb{F}_{p}[E] /\left(E^{p}\right)$.

\section{The $p$-DG KLR algebra}

\section{$4.1 \quad p$-derivations on the KLR algebra}

The KLR algebras. The KLR algebras associated with any Cartan datum were introduced in [20, 21, 41] as a generalization of nilHecke algebras to categorify one-half of the corresponding quantum group.

Let $\Gamma$ be an oriented simply-laced quiver whose vertices are indexed by $I$ ( $I$ is also referred to as the set of colors). Such a quiver determines a Cartan inner product $\cdot: \mathbb{Z}^{I} \times \mathbb{Z}^{I} \longrightarrow \mathbb{Z}$ defined by $i \cdot i=2$ for all $i \in I, i \cdot j=-1$ if $i$ and $j$ are connected by one edge, and $i \cdot j=0$ otherwise. The KLR $\mathbb{k}$-algebra $R(\Gamma)$ associated to $\Gamma$ has the following local diagrammatic presentation. It is generated by braid-like planar diagrams with strands colored by $I$ and carrying dots, subject to the following list of local relations.

- The usual nilHecke relations among same-color crossings and dots.

- Dots slide through crossings of different colors, i. e. for $i, j \in I$ with $i \neq j$,

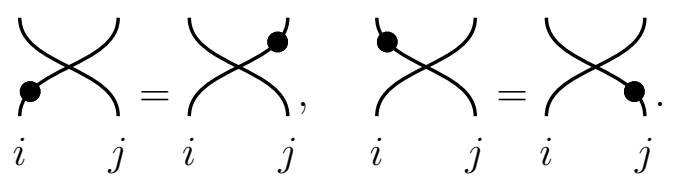


- Reidemeister II type relations between strands,

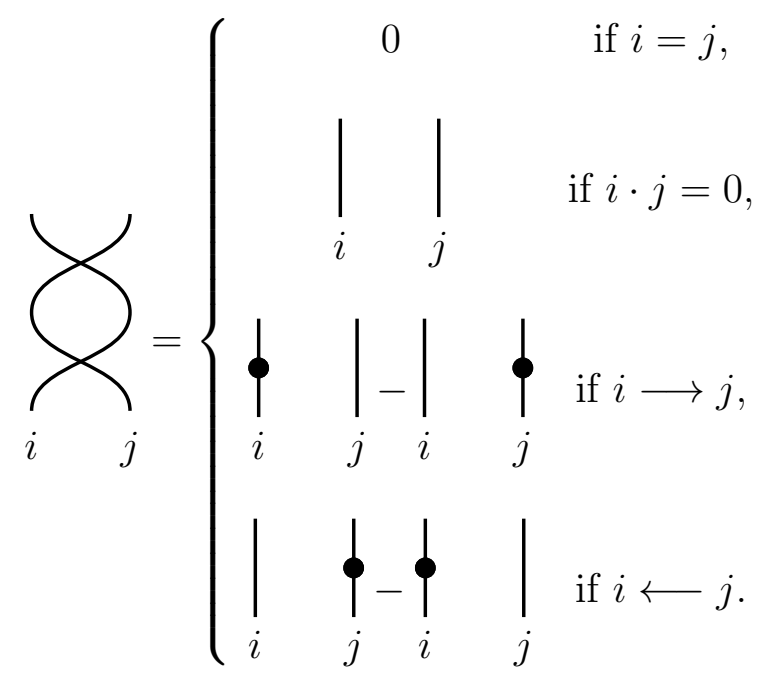

- Reidemeister III type relations

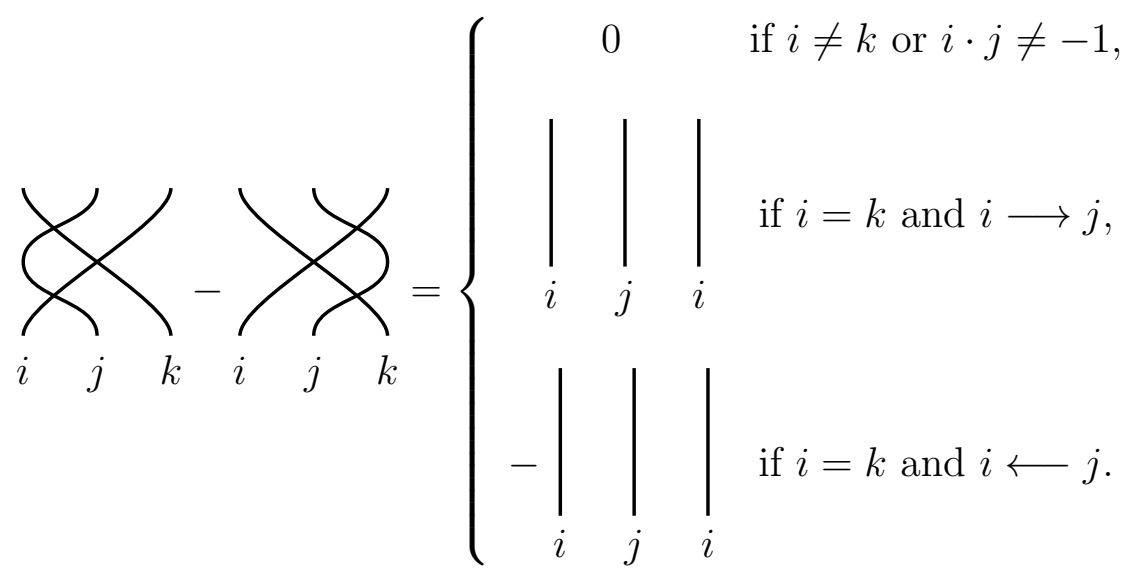

$R(\Gamma)$ naturally decomposes into blocks $R(\Gamma)=\oplus_{\nu \in \mathbb{N}[I]} R(\nu)$, where $\nu=\sum_{i \in I} \nu_{i} i$ are called weights. Each $R(\nu)$ is spanned by diagrams consisting of $|\nu|:=\sum_{i \in I} \nu_{i}$ strands, $\nu_{i}$ of which are colored by $i$. For each weight $\nu=\sum_{i \in I} \nu_{i} i \in \mathbb{N}[I]$, let $\operatorname{Seq}(\nu)$ be the set of sequences of colors $\mathbf{i} \in I^{m}$, where $m=|\nu|$. There are idempotents $1_{\mathbf{i}} \in R(\nu)$ for each $\mathbf{i}=\left(i_{1}, i_{2}, \ldots, i_{m}\right) \in \operatorname{Seq}(\nu)$, depicted by

$$
1_{\mathbf{i}}:=\left.\left.\left.\right|_{i_{1}}\right|_{i_{2}} \ldots\right|_{i_{m}} \ldots
$$

and, as a graded vector space, $R(\nu)=\oplus_{\mathbf{i}, \mathbf{j} \in \operatorname{Seq}(\nu) \mathbf{i}} R(\nu)_{\mathbf{j}}$, where $\mathbf{i}_{\mathbf{i}} R(\nu)_{\mathbf{j}}:=1_{\mathbf{i}} R(\nu) 1_{\mathbf{j}}$.

For more details about these rings, see [20, 21, 41]. Notice that in the previous section we labelled strands by numbers $1,2, \ldots$ starting from top left of a diagram. 
Now we suppress this notation and only keep track of the strands' colors, elements of $I$, written at the bottom endpoints of strands.

The algebra $R(\Gamma)$ has the following symmetries extending those of nilHecke subalgebras. Reflecting a diagram about a horizontal axis induces an algebra anti-involution of $R(\Gamma)$, denoted $\psi$ :

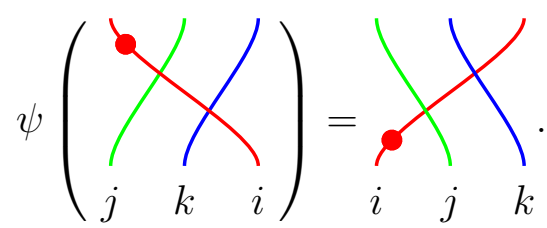

Reflecting a diagram about a vertical axis and simultaneously multiplying it by $(-1)$ for each same-color crossing induces an algebra involution of $R(\Gamma)$, denoted $\sigma$ :

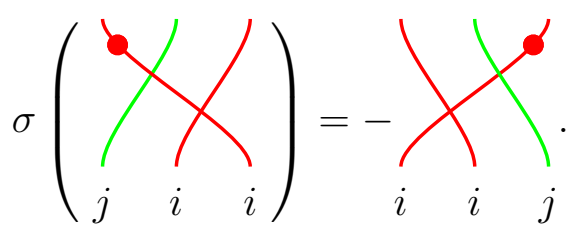

The KLR algebra $R(\Gamma)$ acts faithfully on a direct sum of polynomial spaces, see [20, Section 2.3] for more details. The symmetric group $S_{m}$ acts on $I^{m}$ by permutations of sequences. Define a vector space

$$
\mathcal{P}_{\nu}:=\bigoplus_{\mathbf{i} \in \operatorname{Seq}(\nu)} \mathcal{P}_{\mathbf{i}}, \quad \mathcal{P}_{\mathbf{i}}:=\mathbb{k}\left[x_{1}(\mathbf{i}), \ldots, x_{m}(\mathbf{i})\right] \cdot 1_{\mathbf{i}}
$$

$\mathcal{P}_{\nu}$ inherits an action of $S_{m}$ by setting $w\left(x_{t}(\mathbf{i})\right)=x_{w(t)}(w(\mathbf{i}))$ for any $1 \leq t \leq m$. The algebra $R(\nu)$ acts on $\mathcal{P}_{\nu}$ as follows. First off, ${ }_{\mathbf{j}} R(\nu)_{\mathbf{i}}$ acts on $\mathcal{P}_{\mathbf{k}}$ by zero unless $\mathbf{i}=\mathbf{k}$ and takes $\mathcal{P}_{\mathbf{k}}$ to $\mathcal{P}_{\mathbf{j}}$ when $\mathbf{i}=\mathbf{k}=\left(i_{1}, \ldots, i_{t}, i_{t+1}, \ldots, i_{m}\right)$. The local generators of $R(\nu)$

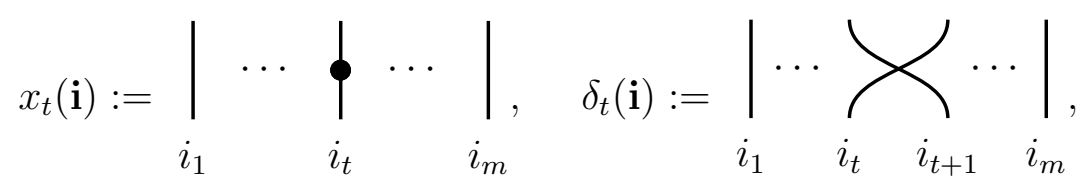

act respectively on $f \in \mathcal{P}_{\mathbf{i}}$ by multiplying $f$ by $x_{t}(\mathbf{i})$ and

$$
\delta_{t}(\mathbf{i})(f):= \begin{cases}{ }^{t} f & \text { if } i_{t} \cdot i_{t+1}=0, \\ \frac{f-{ }^{t} f}{x_{t}(\mathbf{i})-x_{t+1}(\mathbf{i})} & \text { if } i_{t}=i_{t+1}, \\ \left(x_{t+1}\left(s_{k}(\mathbf{i})\right)-x_{t}\left(s_{k}(\mathbf{i})\right)\right) \cdot\left({ }^{t} f\right) & \text { if } i_{t} \longrightarrow i_{t+1}, \\ { }^{t} f & \text { if } i_{t} \longleftarrow i_{t+1},\end{cases}
$$


where ${ }^{t} f$ denotes $f$ with the $t$-th and $(t+1)$-st colored-variables switched. One should not confuse the polynomial representation with the polynomial subalgebra sitting naturally inside $R(\nu)$. In particular, the lowest degree element of $\mathcal{P}_{\mathbf{i}}$, unique up to a non-zero constant and denoted by $1_{\mathbf{i}}$, should not be confused with the idempotent $1_{\mathbf{i}} \in{ }_{\mathbf{i}} R(\nu)_{\mathbf{i}}$.

Example $4.1\left(A_{2}\right)$. For the quiver

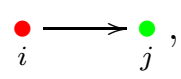

the $R(\nu)$-relations translate into

- The usual nilHecke relations among strands and dots of the same color.

- Dots slide through $i-j$ or $j-i$ crossings:
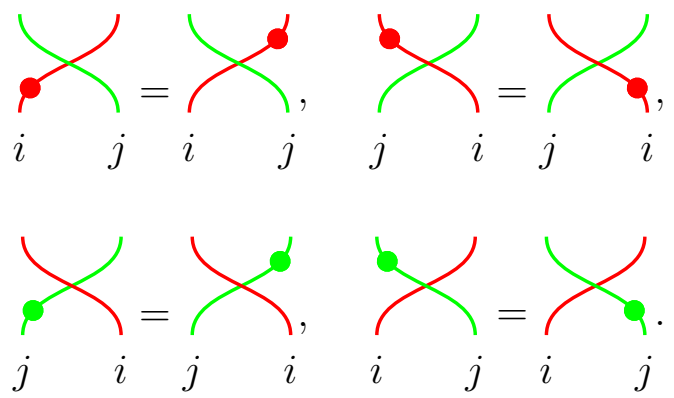

- Two additional Reidemeister II type relations for the new crossings:

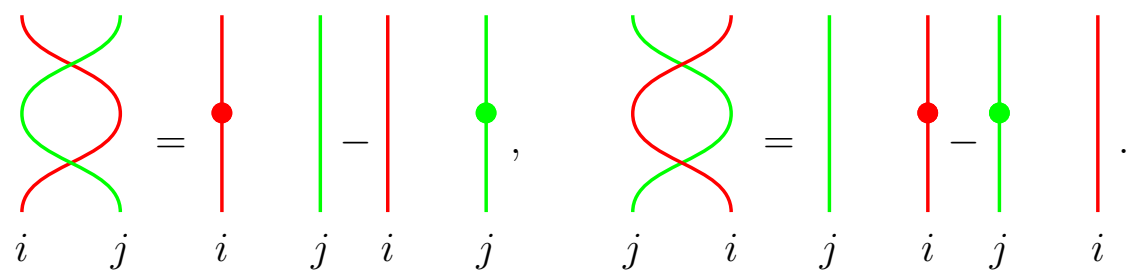

- Two special Reidemeister III type relations:

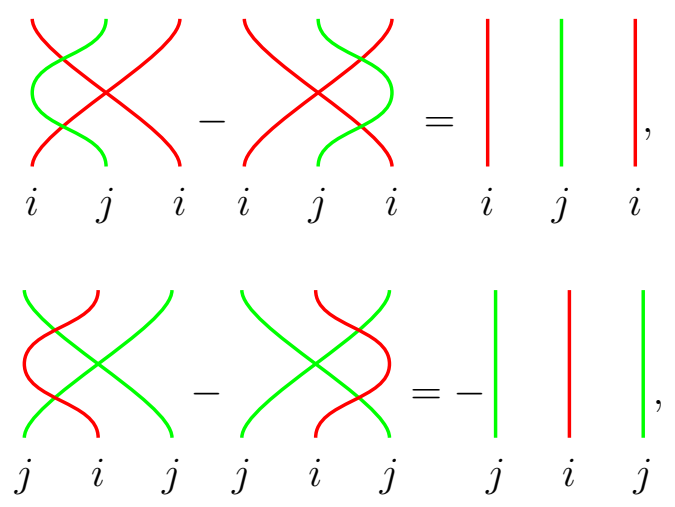


and the other Reidemeister III type relations:
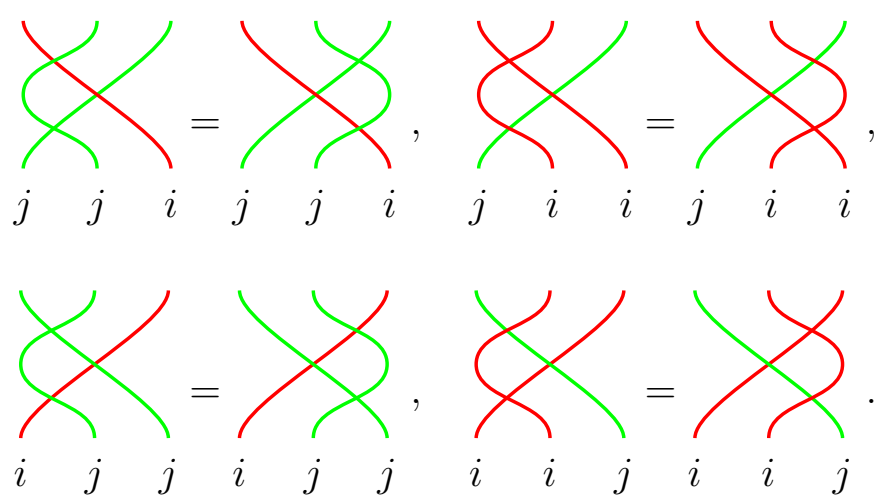

Example $4.2\left(A_{1} \times A_{1}\right)$. This corresponds to the quiver with two vertices and no arrows:

\section{$\stackrel{\bullet}{i} \quad \stackrel{\bullet}{k}$}

Such pairs of vertices in a Dynkin diagram are referred to as distant vertices. The relations for this case are listed below.

- The usual nilHecke relations among strands and dots of the same color.

- Dots slide through $i-k$ and $k-i$ crossings:

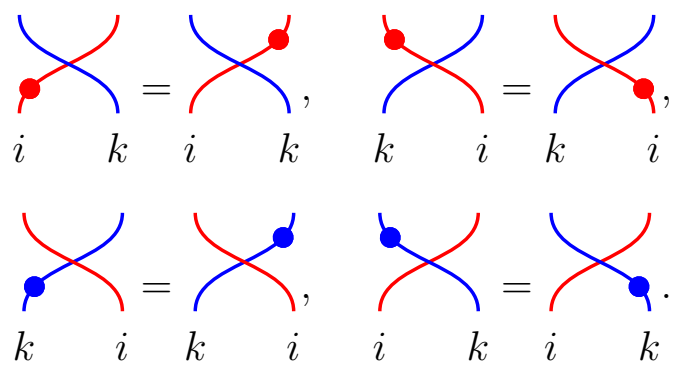

- Two additional Reidemeister II type relations for the new crossings:

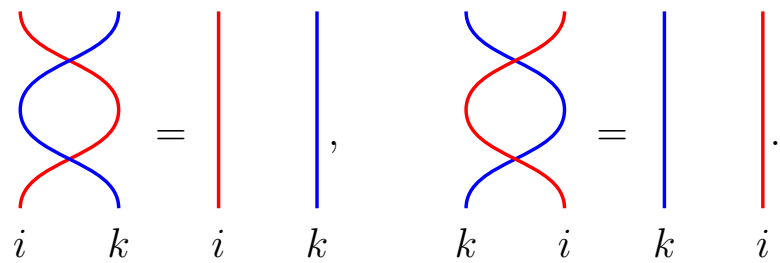

- Reidemeister III type relations:
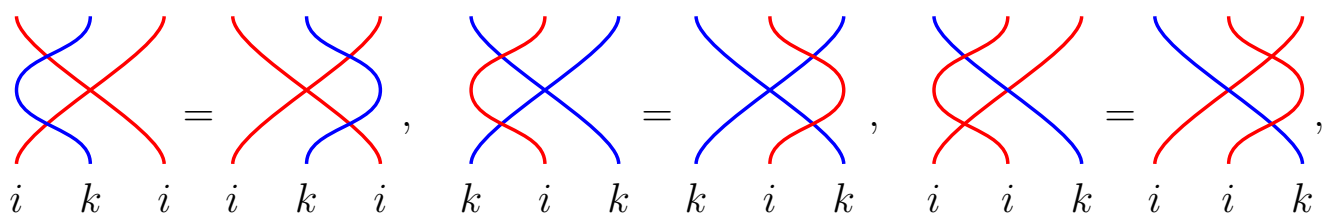

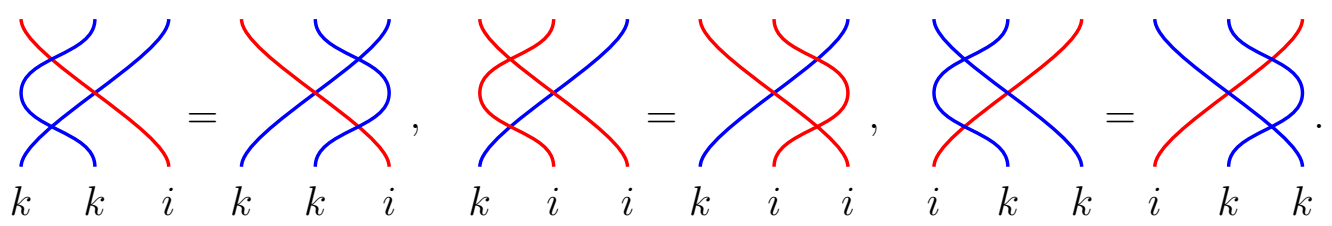

Differentials on KLR algebras. The polynomial representation $\mathcal{P}_{\nu}$ of $R(\nu)$ has a $p$-DG module structure specified as follows. Firstly, let $\mathrm{Pol}_{\nu}:=\mathcal{P}_{\nu}$ with the obvious algebra structure together with with the $p$-differential defined by $\partial\left(x_{t}(\mathbf{i})\right)=x_{t}^{2}(\mathbf{i})$ for all $\mathbf{i} \in \operatorname{Seq}(\nu)$ and $1 \leq t \leq|\nu| .\left(\operatorname{Pol}_{\nu}, \partial\right)$ is then a $p$-DG algebra. Choose a family of linear polynomials $g_{\alpha}(\mathbf{i}):=\sum_{t=1}^{m} \alpha_{t}(\mathbf{i}) x_{t}(\mathbf{i})$, one for each $\mathbf{i} \in \operatorname{Seq}(\nu)$, where $\alpha_{t}(\mathbf{i}) \in \mathbb{F}_{p}$. Let $\partial_{\alpha}$ act on an element $f 1_{\mathbf{i}} \in \mathcal{P}_{\mathbf{i}}$ by

$$
\partial_{\alpha}\left(f 1_{\mathbf{i}}\right):=\partial(f) 1_{\mathbf{i}}+f g_{\alpha}(\mathbf{i}) 1_{\mathbf{i}} .
$$

We denote this $p$-DG $\mathrm{Pol}_{\nu}$-module by $\mathcal{P}_{\nu}(\alpha)$. Unlike in the $A_{1}$ case, we do not make a particular (balanced) choice of the degree to assign to $1_{\mathbf{i}} \in \mathcal{P}_{\nu}(\alpha)$. This degree will depend on $\mathbf{i}$, and will be uniquely determined by the choice of degree for any single sequence of labels in $\operatorname{Seq}(\nu)$.

As in the $A_{1}$ case, a $p$-differential on $\mathcal{P}_{\nu}$ induces a natural $p$-differential on the endomorphism algebra $R(\nu)$. We first investigate these differentials on KLR algebras associated to the only two simply-laced rank-two Cartan data: $A_{2}$ and $A_{1} \times A_{1}$, starting with the $A_{2}$ case.

Consider the polynomial module $\mathcal{P}_{i+j} \cong \mathbb{k}\left[x_{1}, x_{2}\right] 1_{i j} \oplus \mathbb{k}\left[x_{1}, x_{2}\right] 1_{j i}$ (we use the shorthand notation $x_{1}$ for $x_{1}(i j)$ and $x_{1}(j i)$ etc.). Let

$$
\partial_{\alpha}\left(1_{i j}\right)=\alpha_{1}(i j) x_{1} 1_{i j}+\alpha_{2}(i j) x_{2} 1_{i j}, \quad \partial_{\alpha}\left(1_{j i}\right)=\alpha_{1}(j i) x_{1} 1_{j i}+\alpha_{2}(j i) x_{2} 1_{j i} .
$$

An easy computation shows that, diagrammatically, the induced differential on $R(i+j)$ is given on the $i-j$ and $j-i$ crossings by

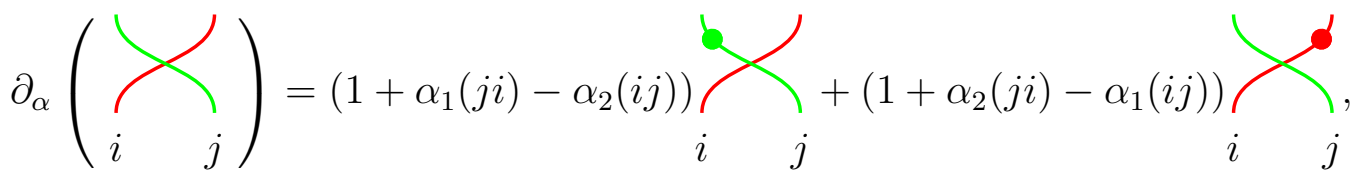

$$
\begin{aligned}
& \left.\left.\partial_{\alpha}\left(\sum_{j}\right)=\left(\alpha_{1}(i j)-\alpha_{2}(j i)\right)\right\rangle_{j}^{b}+\left(\alpha_{2}(i j)-\alpha_{1}(j i)\right)\right\rangle_{j}^{d}
\end{aligned}
$$

We would like the differential to be local, as in Section 3 and in the sense explained earlier. Let $r_{i j}:=1+\alpha_{1}(j i)-\alpha_{2}(i j)$ and $r_{j i}:=\alpha_{1}(i j)-\alpha_{2}(j i)$. Define a four-parameter family of differentials $\partial=\partial\left(a_{i}, a_{j}, r_{i j}, r_{j i}\right)$ on $R\left(\bullet_{i} \rightarrow \underset{j}{\bullet}\right)$ as follows:

$$
\partial\left(\begin{array}{l}
\downarrow \\
i
\end{array}\right)=\oint_{i}^{2}, \quad \partial\left(\begin{array}{l}
\downarrow \\
j
\end{array}\right)=\oint_{j}^{2},
$$




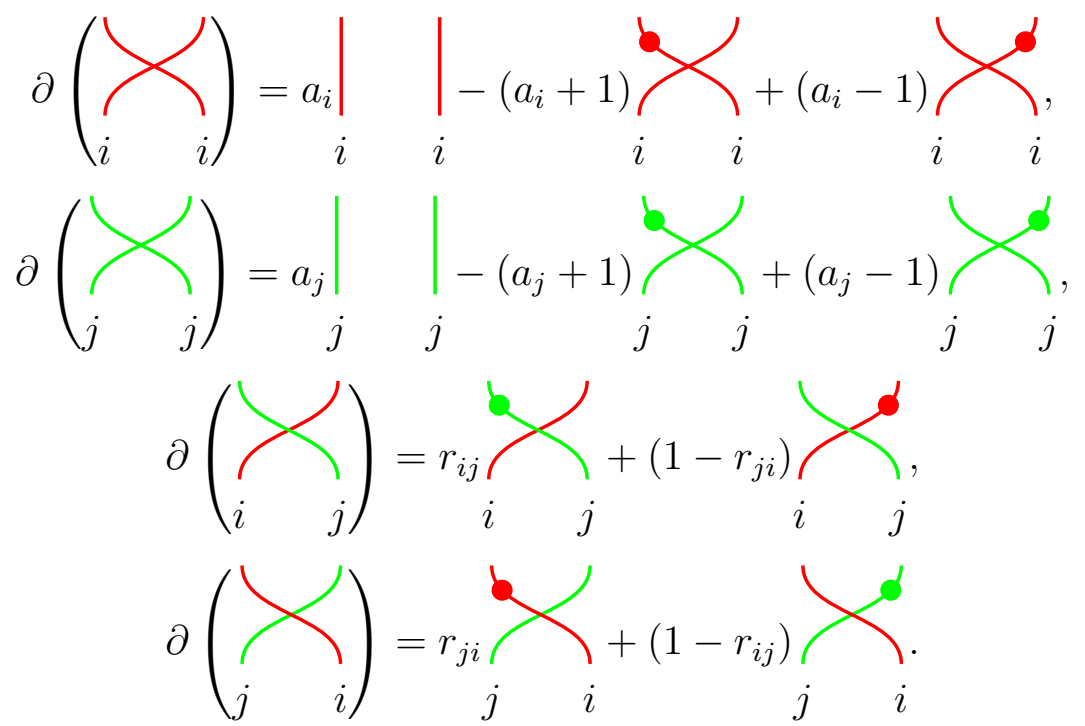

In this definition we allow the parameters $a_{i}, a_{j}, r_{i j}, r_{j i} \in \mathbb{k}$.

Lemma 4.3. The above differential $\partial=\partial\left(a_{i}, a_{j}, r_{i j}, r_{j i}\right)$ satisfies $\partial^{p}=0$ on $R\left(\underset{i}{\bullet} \rightarrow_{j}^{\bullet}\right)$ if and only if $a_{i}, a_{j}, r_{i j}, r_{j i} \in \mathbb{F}_{p}$. Any homogeneous degree two p-nilpotent local derivation on $R\left(\bullet_{i} \rightarrow{ }_{j}\right)$ is of the form $\lambda \cdot \partial\left(a_{i}, a_{j}, r_{i j}, r_{j i}\right)$ for some $\lambda \in \mathbb{k}$ and $a_{i}, a_{j}, r_{i j}, r_{j i} \in \mathbb{F}_{p}$.

Sketch of proof. When $a_{i}, a_{j}, r_{i j}, r_{j i} \in \mathbb{F}_{p}, \partial^{p}=0$ on $R\left(\bullet_{i} \rightarrow \underset{j}{\bullet}\right)$ since $\partial$ was induced from a $p$-DG module. Conversely, Lemma 3.6 shows that $\partial^{p}=0$ on the nilHecke algebra generators of the same color $i$ or $j$ if and only if $a_{i}, a_{j} \in \mathbb{F}_{p}$. A similar computation for $\partial^{p}$ as used in that lemma applied to the new generators $\succ_{j}$ and $>_{i}$ restricts $r_{i j}, r_{j i}$ to be in $\mathbb{F}_{p}$. The classification of all possible derivations follows by an easy but lengthy computation.

The second case is that of the quiver with two distant vertices $\left(A_{1} \times A_{1}\right)$.

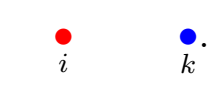

On the polynomial module $\mathcal{P}_{i+k} \cong \mathbb{k}\left[x_{1}, x_{2}\right] 1_{i k} \oplus \mathbb{k}\left[x_{1}, x_{2}\right] 1_{k i}$, set

$$
\partial_{\alpha}\left(1_{i k}\right)=\alpha_{1}(i k) x_{1} 1_{i k}+\alpha_{2}(i k) x_{2} 1_{i k}, \quad \partial_{\alpha}\left(1_{k i}\right)=\alpha_{1}(k i) x_{1} 1_{k i}+\alpha_{2}(k i) x_{2} 1_{k i} .
$$

The induced differential on $R(i+k)$ is given on the $i-k, k-i$ crossings by

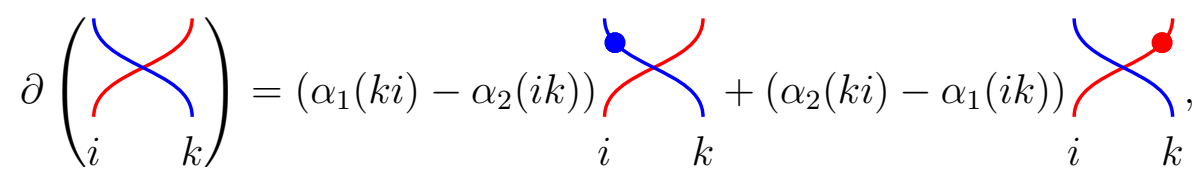




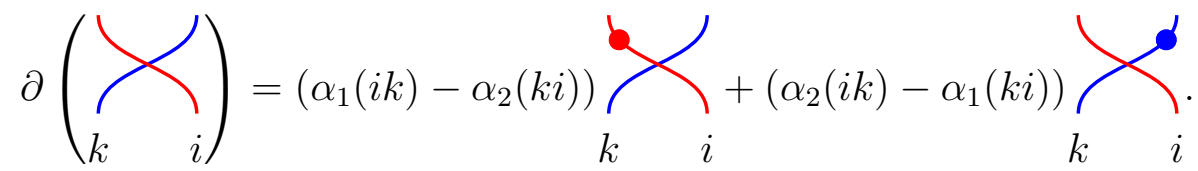

We denote $u_{i k}:=\alpha_{1}(k i)-\alpha_{2}(i k)$ and $u_{k i}:=\alpha_{1}(i k)-\alpha_{2}(k i)$, and require that the differential is independent of the position of the crossings. This results in a six-parameter family $\left(\mu_{i}, \mu_{k}, a_{i}, a_{k}, u_{i k}, u_{k i} \in \mathbb{k}\right)$ of local differentials:

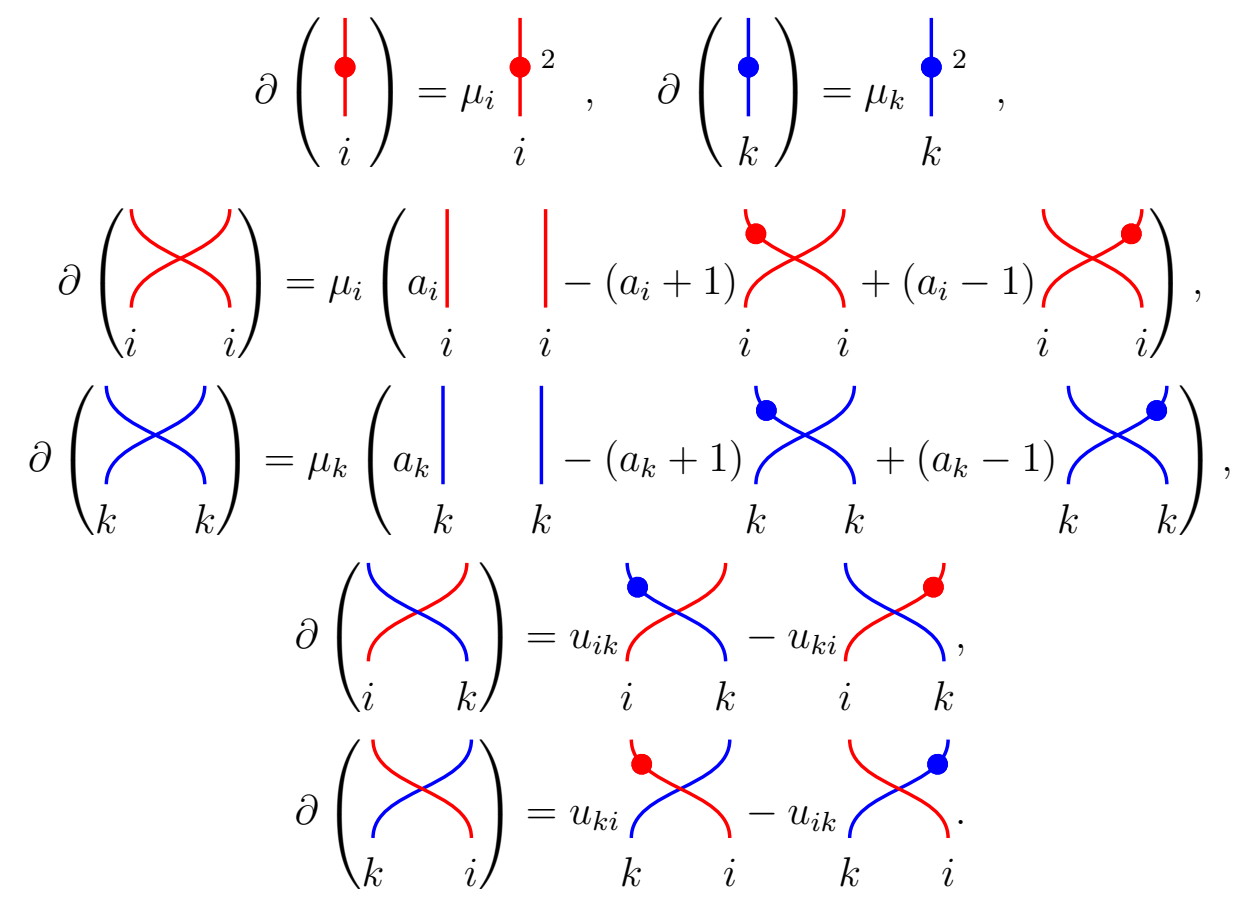

The proof of the following lemma is entirely analogous to those of Lemmas 3.6, 4.3,

Lemma 4.4. The above differential $\partial=\partial\left(a_{i}, a_{k}, u_{i k}, u_{k i}, \mu_{i}, \mu_{k}\right)$ satisfies $\partial^{p}=0$ on $R\left(\dot{\bullet}_{i}^{\bullet} \dot{k}^{\bullet}\right)$ if and only if $a_{i}, a_{k}, u_{i k}, u_{k i} \in \mathbb{F}_{p}$ while $\mu_{i}, \mu_{k} \in \mathbb{k}$. Any homogeneous degree two p-nilpotent local derivation on $R\left(\begin{array}{ll}\bullet & \bullet\end{array}\right)$ arises in this way.

Remark 4.5. By rescaling the differential on the nilHecke algebra, one could have specified that $\partial(x(i))=\mu_{i} x^{2}(i)$ by any $\mu_{i} \in \mathbb{k}$ for each color $i \in I$, and $\partial(\delta(i))$ would then be modified accordingly (as above, for the $i-i$ and $k-k$ crossings). Inside the KLR algebra, one might attempt to rescale the nilHecke differentials for each color separately. This can be accomplished when the colors are distant, but for adjacent colors the $A_{2}$ relations force the scalings to be the same. Therefore, in a connected simply-laced quiver, we may and will always assume that the scaling factor $\mu_{i}=1$ for each color $i \in I$.

In a similar way as in Proposition 3.9, one shows that the symmetries $\psi, \sigma$ of $R(\Gamma)$ intertwine differentials. 
Proposition 4.6. The following relations on the differentials hold.

(i) $\operatorname{On} R(\stackrel{\bullet}{\bullet} \rightarrow \underset{j}{\bullet})$,

$$
\begin{gathered}
\psi \circ \partial\left(a_{i}, a_{j}, r_{i j}, r_{j i}\right) \circ \psi=\partial\left(-a_{i},-a_{j}, 1-r_{i j}, 1-r_{j i}\right), \\
\sigma \circ \partial\left(a_{i}, a_{j}, r_{i j}, r_{j i}\right) \circ \sigma=\partial\left(-a_{i},-a_{j}, 1-r_{i j}, 1-r_{j i}\right) .
\end{gathered}
$$

(ii) $\operatorname{On} R\left(\begin{array}{ll}\bullet & \bullet\end{array}\right)$,

$$
\begin{gathered}
\psi \circ \partial\left(a_{i}, a_{k}, u_{i k}, u_{k i}, \mu_{i}, \mu_{k}\right) \circ \psi=\partial\left(-a_{i},-a_{k},-u_{i k},-u_{k i}, \mu_{i}, \mu_{k}\right), \\
\sigma \circ \partial\left(a_{i}, a_{k}, u_{i k}, u_{k i}, \mu_{i}, \mu_{k}\right) \circ \sigma=\partial\left(-a_{i},-a_{k},-u_{i k},-u_{k i}, \mu_{i}, \mu_{k}\right) .
\end{gathered}
$$

Sketch of proof. To show these relations, it suffices to check them on the algebra generators. The proof is entirely similar to the argument in the proof of Proposition 3.9. That $a_{i}, a_{j}, a_{k}$ are replaced respectively by $-a_{i},-a_{j},-a_{k}$ under conjugation is implied by the proposition. For $r_{i j}, r_{j i}, u_{i k}, u_{k i}$ it suffices to compute both sides on two-color crossings. For instance, denoting $\partial\left(a_{i}, a_{j}, r_{i j}, r_{j i}\right)$ by $\partial_{r, s}$ for short, where $r=r_{j i}$ and $s=r_{i j}$, we have

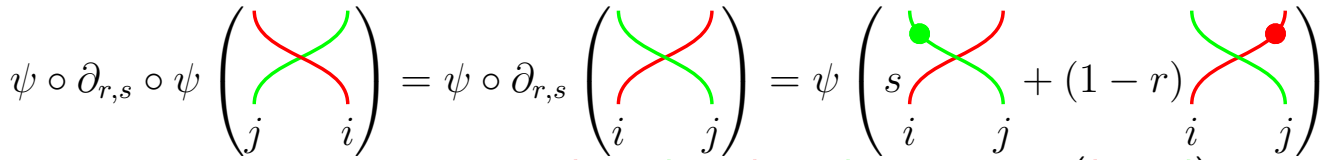

$$
\begin{aligned}
& \left.=(1-r) \sum_{j}^{b}+s\right\rangle_{j}^{d}=\partial_{1-r, 1-s}\left(\sum_{j}\right) \text { ). }
\end{aligned}
$$

The rest of the proof follows similarly.

The proof of the following result is similar to that of the nilHecke case (Proposition 3.10).

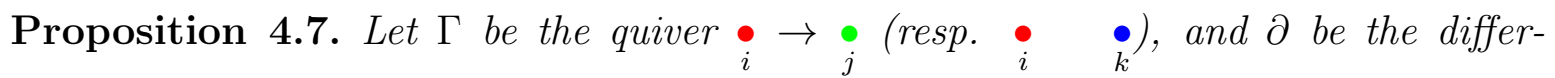
ential $\partial\left(a_{i}, a_{j}, r_{i j}, r_{j i}\right)$ (resp. $\partial\left(a_{i}, a_{k}, u_{i k}, u_{k i}, \mu_{i}, \mu_{k}\right)$ with $\left.\mu_{i}, \mu_{k} \neq 0\right)$. Then for each weight $\nu \in \mathbb{N}[I]$, where $I=\{i, j\}$ (resp. $I=\{i, k\}$ ), the p-complex $(R(\nu), \partial)$ is quasiisomorphic to a finite dimensional p-complex.

Sketch of proof. Since the differentials either preserve or decrease the number the crossings in a diagram, one can filter the $p$-complex $R(\nu)$ by the number of crossings in the diagrams of a basis, so that each subquotient is isomorphic to a rank-one polynomial module over the $p$-DG algebra $\mathbb{k}\left[x_{1}, \cdots, x_{m}\right]$ with $\partial\left(x_{i}\right)=x_{i}^{2}$. Such polynomial modules are quasi-isomorphic to finite dimensional $p$-complexes by the discussion in Section 3.1 (after equation (321)). 
Extension to any simply-laced Cartan datum. Now let $\Gamma$ be a connected, simplylaced Cartan datum with vertex set $I$. Such data are classified by finite graphs without loops at vertices or multiple edges. Fix an arbitrary orientation for $\Gamma$. Define a multiparameter family of differentials

$$
\partial:=\partial\left(a_{i}, r_{i j}, r_{j i}, u_{i k}, u_{k i}\right)
$$

where $i, j, k \in I$, on $R(\Gamma)$ as follows.

(i) For each vertex $i \in I$ choose $a_{i} \in \mathbb{F}_{p}$ and define

$$
\partial\left(\sum_{i}\left(\begin{array}{l}
\boldsymbol{\phi} \\
i
\end{array}\right)=\left.a_{i}\right|_{i} ^{2}\right.
$$

(ii) For each pair of vertices $i, j \in I$ in $\Gamma$ connected by an oriented edge $i \rightarrow j$ choose $r_{i j}, r_{j i} \in \mathbb{F}_{p}$ and define

$$
\partial\left(\sum_{i} \sum_{j}\right)=r_{i j} \sum_{i}
$$

(iii) For each unordered pair of disconnected vertices $i, k \in I$ choose $u_{i k}, u_{k i} \in \mathbb{F}_{p}$ and define

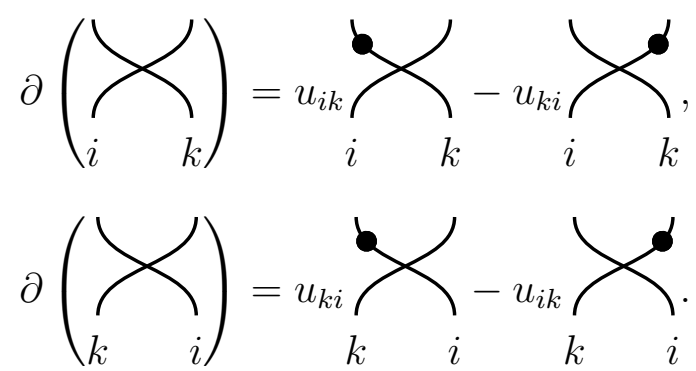


This assignment gives rises to a differential on $R(\Gamma)$. We have already checked that the defining relations in $R(\Gamma)$ involving strands of at most two colors are respected by these differentials. It is immediate to see that the only missing relations

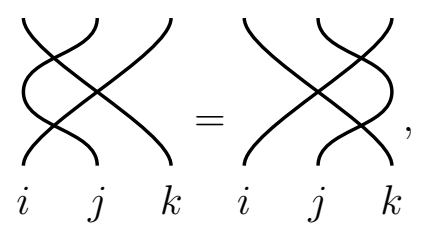

where $i, j, k$ are pairwise distinct vertices, are preserved under the differential. In these relations, the types of distinct-color crossings involved do not change-only their orders do; hence the differential is well-defined.

\subsection{Quantum Serre relations}

Given any simply-laced Cartan datum $\Gamma$ with vertex set $I$, there is an associated (half) quantum Kac-Moody algebra $\mathbf{f}_{\Gamma}[29$, Section 1], built as follows. One starts with a free $\mathbb{N}[I]$-graded associative algebra ' $\mathbf{f}_{\Gamma}$ over the field $\mathbb{Q}(q)$ with generators $E_{i}$ for all $i \in I$, where $\operatorname{deg}\left(E_{i}\right)=i$ as an element of $\mathbb{N}[I]$. Then $\mathbf{f}_{\Gamma}$ is defined to be ${ }^{\prime} \mathbf{f}_{\Gamma}$ quotiented by the two-sided ideal generated by the following relations, known as the quantum Serre relations:

$$
E_{i} E_{k}=E_{k} E_{i}
$$

if $i, k$ are distant vertices, while

$$
\left\{\begin{array}{l}
{[2] E_{i} E_{j} E_{i}=E_{i} E_{i} E_{j}+E_{j} E_{i} E_{i},} \\
{[2] E_{j} E_{i} E_{j}=E_{j} E_{j} E_{i}+E_{i} E_{j} E_{j},}
\end{array}\right.
$$

if $i, j$ are connected by one edge. The divided powers $E_{i}^{(n)}:=E_{i}^{n} /[n]$ ! for all $i \in I$ and $n \in \mathbb{N}$ generate an $\mathbb{Z}\left[q, q^{-1}\right]$ integral subalgebra $\mathbf{f}_{\Gamma, \mathbb{Z}}$ of $\mathbf{f}_{\Gamma}$ such that

$$
\mathbf{f}_{\Gamma, \mathbb{Z}} \otimes_{\mathbb{Z}\left[q, q^{-1}\right]} \mathbb{Q}(q)=\mathbf{f}_{\Gamma} .
$$

The relations (104) acquire the divided power form

$$
\left\{\begin{array}{l}
E_{i} E_{j} E_{i}=E_{i^{(2)}} E_{j}+E_{j} E_{i^{(2)}}, \\
E_{j} E_{i} E_{j}=E_{j^{(2)}} E_{i}+E_{i} E_{j^{(2)}} .
\end{array}\right.
$$

We will abbreviate $E_{i} E_{j} E_{i}$ by $E_{i j i}, E_{i(2)} E_{j}$ by $E_{i(2)}$ etc. in what follows.

The main goal of this section is to show that requiring these relations on the Grothendieck groups of the derived categories of $p$-DG algebras $(R(\nu), \partial)$ severely restricts possible choices of parameters in $\partial$ (parameters $a_{i}, r_{i j}, r_{j i}, u_{i k}, u_{k i} \in \mathbb{F}_{p}$ ). We will show at the end that for such special parameter values the quantum Serre relations hold on the level of $K_{0}$, interpreting these relations on the categorical level as well. The 
local nature of the differentials allows us to consider the two special cases $A_{1} \times A_{1}$ and $A_{2}$ separately (see Examples 4.1 and 4.2).

Below we will use that if $\sum_{\alpha \in J}\left[M_{\alpha}\right]=\sum_{\beta \in K}\left[N_{\beta}\right]$ holds in the Grothendieck group $K_{0}(R(\Gamma), \partial)$, where $M_{\alpha}, N_{\beta}$ and $P$ are compact $p$-DG modules over $(R(\Gamma), \partial)$ and $J, K$ are some finite index sets, then

$$
\sum_{\alpha \in J}\left[\mathbf{R H O M}_{R(\Gamma)}\left(P, M_{\alpha}\right)\right]=\sum_{\beta \in K}\left[\mathbf{R H O M}_{R(\Gamma)}\left(P, N_{\beta}\right)\right]
$$

in $K_{0}(H-\underline{\bmod }) \cong \mathbb{O}_{p}$. This follows since

$$
\operatorname{RHOM}_{R(\Gamma)}(-,-): \mathcal{D}^{c}(R(\Gamma), \partial) \times \mathcal{D}^{c}(R(\Gamma), \partial) \longrightarrow H-\underline{\mathrm{fmod}}
$$

is an exact bi-functor, and $R(\Gamma)$ has finite dimensional cohomology (Proposition 4.7) under the differentials defined by equations (97)-(102). When the context is clear, we will just write $\mathbf{R H O M}(-,-)$ for short.

The $A_{1} \times A_{1}$ case. Consider the $p$-DG algebra $\left(R(i+k), \partial\left(a_{i}, a_{k}, u_{i k}, u_{k i}\right)\right)$. Define the $p$-DG modules

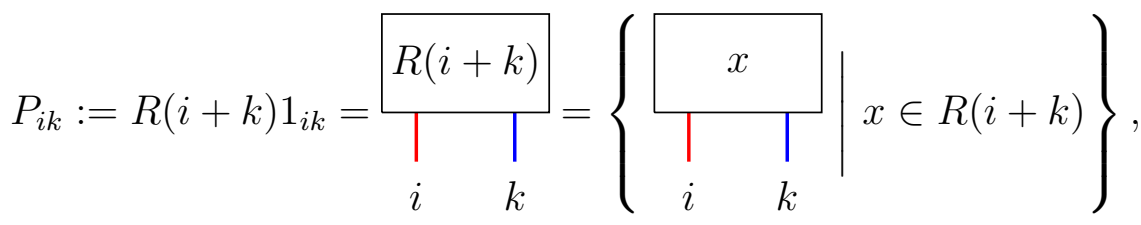

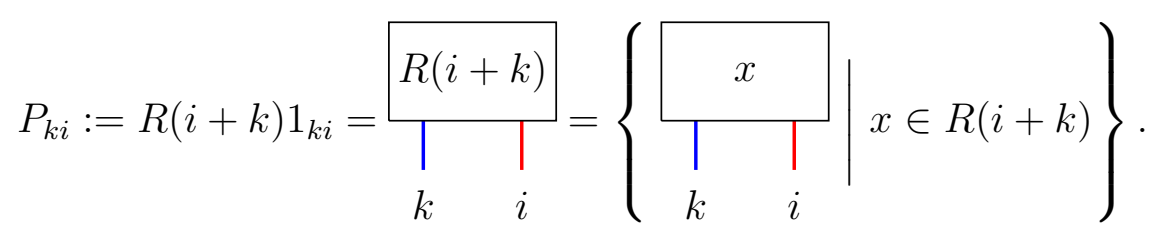

Both modules are cofibrant since they are $p$-DG direct summands of the free module $R(i+k)$ (Proposition 2.24). When the parameters $u_{i k}=u_{k i}=0$ in the definition of the differential, there are isomorphisms of $p$-DG modules

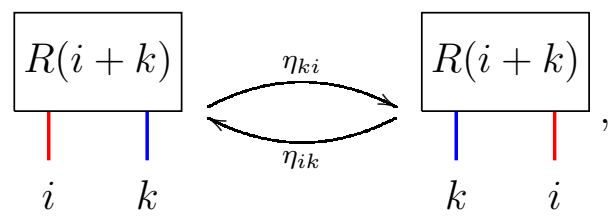

where $\eta_{k i}$ is given by post-composing any diagram in $P_{i k}$ with the crossing $\succ_{k}$, since $\partial\left(\gtrless_{k} \gamma_{i}\right)=0$ so that multiplication by this element commutes with the differential. 
Likewise $\eta_{i k}$ is given by attaching the crossing $\overbrace{i}$ to the bottom of any diagram of $P_{k i}$. Therefore, in $K_{0}\left(R(i+k), \partial\left(a_{i}, a_{k}, 0,0\right)\right),\left[P_{i k}\right]=\left[P_{k i}\right]$. We interpret the $p$-DG isomorphism $P_{i k} \cong P_{k i}$ as a categorical lift of the relation $E_{i} E_{k}=E_{k} E_{i}$. The next result shows that such a categorical interpretation only exists when $u_{i k}=u_{k i}=0$.

Proposition 4.8. In the Grothendieck group $K_{0}\left(R(i+k), \partial\left(a_{i}, a_{k}, u_{i k}, u_{k i}\right)\right)$, the relation $\left[P_{i k}\right]=\left[P_{k i}\right]$ holds if and only if $u_{i k}=u_{k i}=0$. Furthermore, when $u_{i k}=u_{k i}=0$, there is isomorphisms of $R(i+k)_{\partial}$-module

$$
\eta_{k i}: P_{i k} \longrightarrow P_{k i}
$$

whose inverse is $\eta_{i k}$.

Proof. For the ease of notation, we will let $u=u_{k i}$ and $v=u_{i k}$ in the proof. If $\left[P_{i k}\right]=\left[P_{k i}\right]$, using the RHOM pairing with the cofibrant modules $P_{i k}$ and $P_{k i}$ (107), we have

$$
\begin{aligned}
& {\left[\mathbf{R H O M}_{R(i+k)}\left(P_{i k}, P_{i k}\right)\right]=\left[\mathbf{R H O M}_{R(i+k)}\left(P_{i k}, P_{k i}\right)\right],} \\
& {\left[\mathbf{R H O M}_{R(i+k)}\left(P_{k i}, P_{i k}\right)\right]=\left[\mathbf{R H O M}_{R(i+k)}\left(P_{k i}, P_{k i}\right)\right] .}
\end{aligned}
$$

The cofibrance condition implies that the derived hom is isomorphic to the usual hom as $H$-modules. On one hand, the left hand side of the equation (108) equals the $\mathbb{O}_{p}$-dimension of the $H$-module

$$
\operatorname{RHOM}\left(P_{i k}, P_{i k}\right) \cong \operatorname{HOM}_{R(i+k)}\left(P_{i k}, P_{i k}\right) \cong \mathbb{k}\left\langle\left.{ }_{i}^{n_{1}} \oint_{k}^{\phi}\right|_{\left.n_{1}, n_{2} \in \mathbb{N}\right\rangle} ^{n_{2}}\right|
$$

which is quasi-isomorphic to the trivial $H$-module $V_{0} \cong \mathbb{k}$ whose $\mathbb{O}_{p}$-dimension (see Notation 2.7) is 1. On the other hand,

$$
\operatorname{RHOM}\left(P_{i k}, P_{k i}\right) \cong \operatorname{HOM}_{R(i+k)}\left(P_{i k}, P_{k i}\right) \cong \mathbb{k}\left\langle\sum_{k}^{n_{1}} \sum_{i}^{n_{2}} \mid n_{1}, n_{2} \in \mathbb{N}\right\rangle,
$$

and the differential $\partial=\partial\left(a_{i}, a_{k}, u, v, \mu_{i}, \mu_{k}\right)$ acts on any basis element by

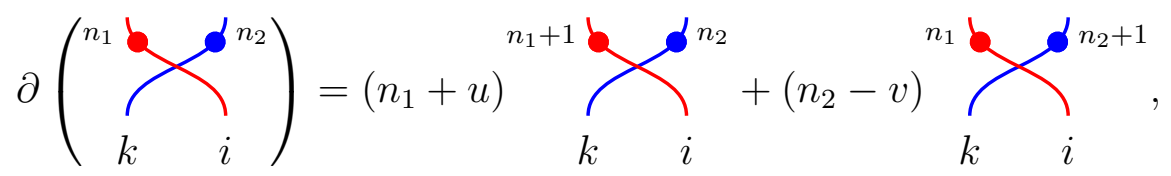

so that as an $H$-module, $\mathbf{R H O M}\left(P_{i k}, P_{k i}\right)$ is quasi-isomorphic to $V_{p-u} \otimes V_{v}$, whose $\mathbb{O}_{p^{-}}$ dimension is $\left[\mathbf{R H O M}\left(P_{i k}, P_{k i}\right)\right]=\left(1+q^{2}+\cdots+q^{2(p-u)}\right)\left(1+q^{2}+\cdots+q^{2 v}\right)$. Therefore we obtain the constraint equation in $\mathbb{O}_{p}$

$$
\left(1+q^{2}+\cdots+q^{2(p-u)}\right)\left(1+q^{2}+\cdots+q^{2 v}\right)=1 .
$$


Likewise, computing the $\mathbb{O}_{p}$-dimension of both sides of equation (109) gives us

$$
\left(1+q^{2}+\cdots+q^{2(p-v)}\right)\left(1+q^{2}+\cdots+q^{2 u}\right)=1 .
$$

Mod $p$ reduction of the equations (110), (111) results in

$$
(1-u)(1+v) \equiv 1, \quad(1-v)(1+u) \equiv 1(\bmod p) .
$$

Solving these last two equations together gives $u=v=0$, as claimed. The last statement follows from the discussion before the proposition.

The $A_{2}$ case. We first review briefly the proof of the quantum Serre relations in the Grothendieck group $K_{0}\left(R\left({ }_{i} \rightarrow \underset{j}{\mathbf{e}}\right)\right)$, as done in [20, Section 2.5]. As an $R(2 i+j)$ module, $P_{i j i}:=R(2 i+j) 1_{i j i}=\left\{\begin{array}{lll}\hline & x & \\ T & 1 & 1 \\ i & j & i\end{array} \mid x \in R(2 i+j)\right\}$ decomposes into a direct sum of projective modules $P_{i j i} \cong P_{i^{(2)} j} \oplus P_{j i(2)}$, where

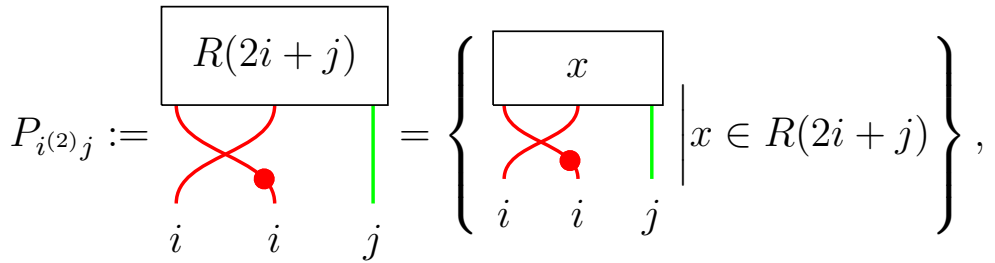

$$
\begin{aligned}
& P_{j i^{(2)}}:=\overbrace{j}^{R(2 i+j)}=\left\{\sum_{i} \sum_{j} \sum_{i} \mid x \in R(2 i+j)\right\} .
\end{aligned}
$$

The isomorphism is realized via the four maps

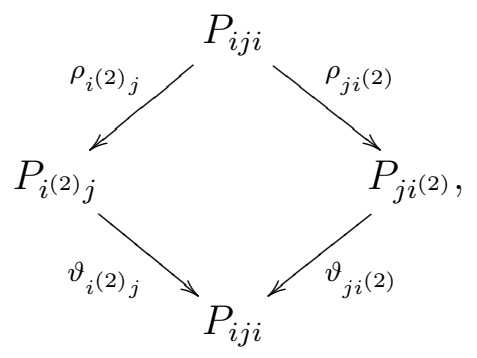


where $\rho_{i(2)}$ is the left $R(2 i+j)$-module map given by right multiplying any element of $P_{i j i}$ by $\sum_{i} \sum_{j}$. We will simply refer to this map as

$$
\rho_{i(2) j}=\bigotimes_{i} \sum_{j}
$$

Likewise, the other maps in the above diagram are given by

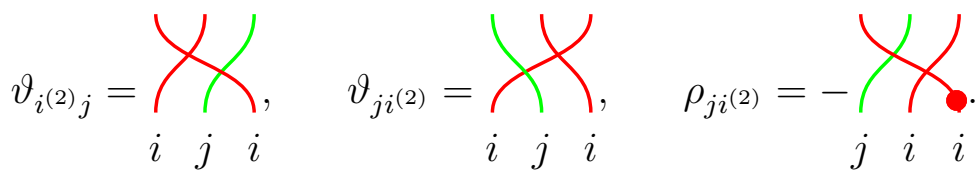

Moreover, under this decomposition, there are isomorphisms of projective $R(2 i+j)$ modules

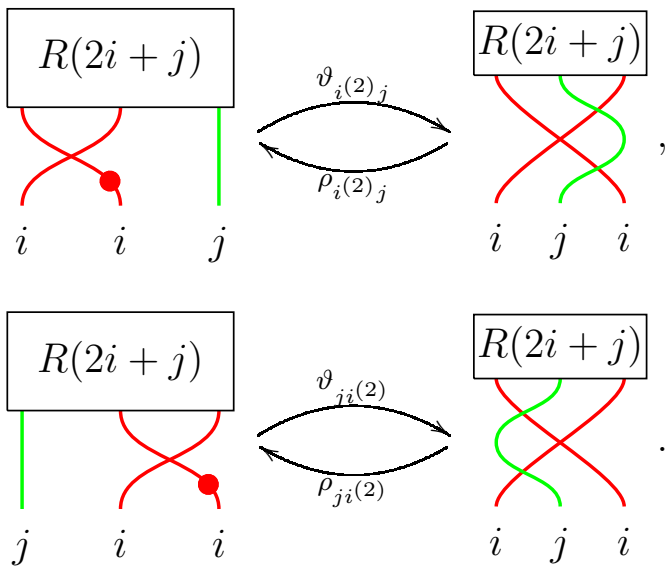

Hence in the usual Grothendieck group of $R(2 i+j)$ spanned by symbols of finitely generated graded projective modules, we have

$$
\left[P_{i j i}\right]=\left[P_{i(2)}\right]+\left[P_{j i(2)}\right]
$$

This is precisely the divided power form of the quantum Serre relation

$$
E_{i j i}=E_{i(2) j}+E_{j i(2)}
$$

Now consider $R(2 i+j)$ as a $p$-DG algebra equipped with the differential $\partial=$ $\partial\left(a_{i}, a_{j}, r_{i j}, r_{j i}\right)$ (see Lemma 4.3). A categorical lifting of the quantum Serre relation to the category of $p$-DG modules over $(R(2 i+j), \partial)$ necessarily means that the following equality of symbols

$$
[2]\left[P_{i j i}\right]=\left[P_{i i j}\right]+\left[P_{j i i}\right]
$$


holds in $K_{0}(R(2 i+j), \partial)$. The modules $P_{i j i}, P_{i i j}, P_{j i i}$ are compact cofibrant, and we compute the following Cartan matrix with entries in $K_{0}(H-\underline{\bmod }) \cong \mathbb{O}_{p}$ :

$$
\left(\begin{array}{lll}
{\left[\operatorname{RHOM}\left(P_{i i j}, P_{i i j}\right)\right]} & {\left[\mathbf{R H O M}\left(P_{i i j}, P_{i j i}\right)\right]} & {\left[\operatorname{RHOM}\left(P_{i i j}, P_{j i i}\right)\right]} \\
{\left[\operatorname{RHOM}\left(P_{i j i}, P_{i i j}\right)\right]} & {\left[\operatorname{RHOM}\left(P_{i j i}, P_{i j i}\right)\right]} & {\left[\operatorname{RHOM}\left(P_{i j i}, P_{j i i}\right)\right]} \\
{\left[\operatorname{RHOM}\left(P_{j i i}, P_{i i j}\right)\right]} & {\left[\operatorname{RHOM}\left(P_{j i i}, P_{i j i}\right)\right]} & {\left[\operatorname{RHOM}\left(P_{j i i}, P_{j i i}\right)\right]}
\end{array}\right) .
$$

We give one example to show how these entries are computed.

Until the end of this case $\left(A_{2}\right)$, we will write $a:=a_{i}, b:=a_{j}, r:=r_{j i}, s:=r_{i j}$ for convenience.

Example 4.9. Consider the $(2,2)$-entry $\left[\operatorname{RHOM}\left(P_{i j i}, P_{i j i}\right)\right]$. Since $P_{i j i}$ is cofibrant, the $p$-complex $\operatorname{RHOM}\left(P_{i j i}, P_{i j i}\right)$ is isomorphic to $\operatorname{HOM}\left(P_{i j i}, P_{i j i}\right) \cong 1_{i j i} R(2 i+j) 1_{i j i}$ with the induced differential from the algebra $R(2 i+j)$ (Proposition 2.24). Diagrammatically,

$$
\begin{aligned}
\operatorname{HOM}\left(P_{i j i}, P_{i j i}\right) & =\mathbb{k}\left\langle\left.\left.\left.\right|_{i} ^{k_{1}}\right|_{j} ^{k_{2}}\right|_{i} ^{k_{3}},\left.\sum_{i}^{k_{1}}\right|_{i} ^{k_{2}} k_{1}^{k_{3}}, k_{2}, k_{3} \in \mathbb{N}\right\rangle \\
& \cong \mathbb{k}\left[x_{1}(i), x_{2}(j), x_{3}(i)\right]\left\langle\left.\left.\right|_{i}||_{i}\right|_{i}\right\rangle .
\end{aligned}
$$

The differential $\partial=\partial(a, b, s, r)$ acts on the polynomial algebra $\operatorname{Pol}_{i j i}:=\mathbb{k}\left[x_{1}(i), x_{2}(j), x_{3}(i)\right]$ by $\partial\left(x_{1}(i)\right)=x_{1}^{2}(i), \partial\left(x_{2}(j)\right)=x_{2}^{2}(j)$, and $\partial\left(x_{3}(i)\right)=x_{3}^{2}(i)$, while on the module basis elements it has the effect

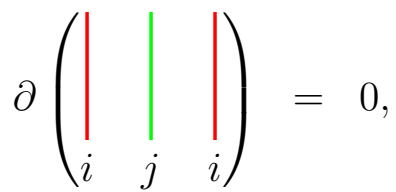

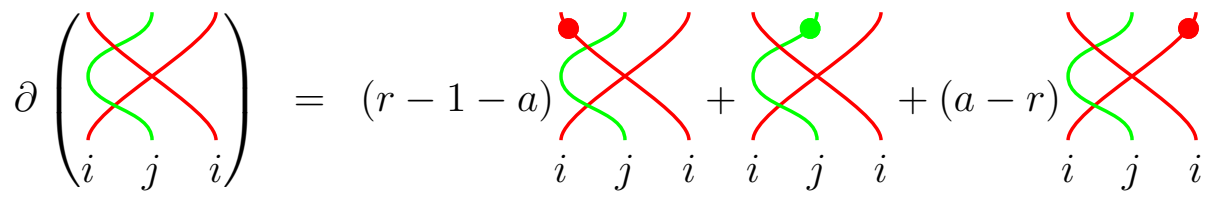

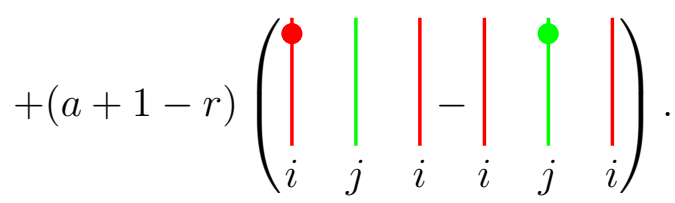


Therefore, as a $p$-DG module over $\left(\mathrm{Pol}_{i j i}, \partial\right), \operatorname{HOM}\left(P_{i j i}, P_{i j i}\right)$ fits into the short exact sequence,

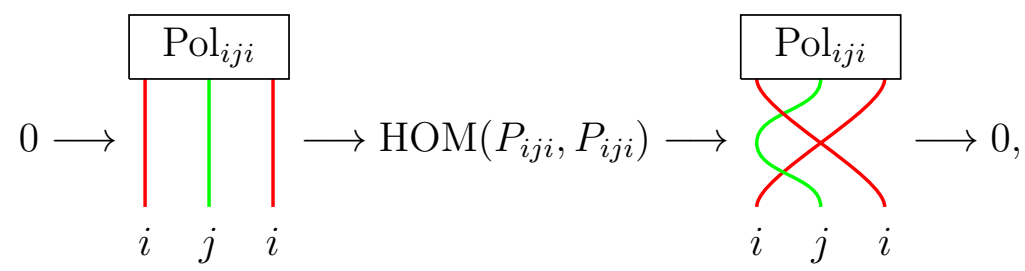

where the two end terms are respectively isomorphic to the $p$-DG Pol $_{i j i}$-modules (ideals) $\mathrm{Pol}_{i j i} \cdot 1$ and $\mathrm{Pol}_{i j i} \cdot\left(x_{1}^{r-1-a}(i) x_{2}(j) x_{3}^{a-r}(i)\right)$. It is easy to see that the former is quasiisomorphic to $\mathbb{k}$, while the latter module is acyclic. In $\mathcal{D}\left(\mathrm{Pol}_{i j i}, \partial\right)$, we have a corresponding distinguished triangle (Lemma 2.20), so that the $\mathbb{O}_{p}$-dimension of $\operatorname{RHOM}\left(P_{i j i}, P_{i j i}\right)$ satisfies

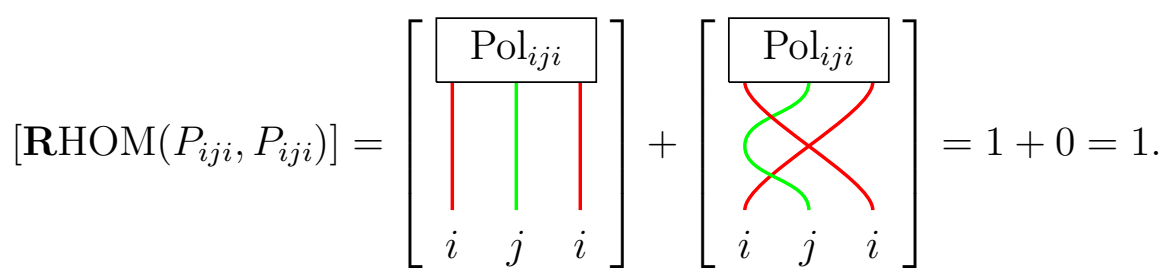

By similar computations as in the above example, one calculates each of the Cartan matrix entries to be

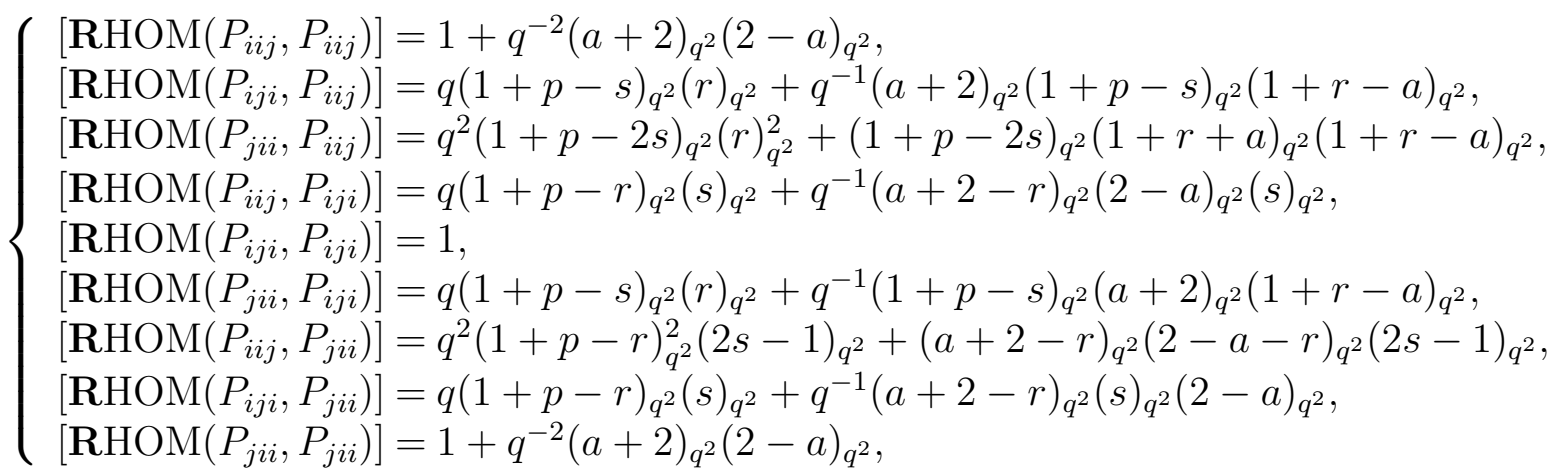

where $(n)_{q^{2}}$ denotes the unbalanced quantum integer $(n)_{q^{2}}:=1+q^{2}+\cdots+q^{2(n-1)} \in \mathbb{O}_{p}$. Equation (107) yields the following $\mathbb{O}_{p}$-vector form of the quantum Serre relation:

$$
[2] \cdot(\text { the second row })=(\text { the first row })+(\text { the third row }) \text {. }
$$

When reduced $\bmod p$, these equations give rise to the system of equations:

$$
\begin{aligned}
& 2(1-s)\left(3 r-a+2-a^{2}+a r\right)=\left(5-a^{2}\right)+(1-2 s)\left(2 r^{2}+2 r+1-a^{2}\right), \\
& 2=s\left(5-3 r-a^{2}+a r\right)+(1-s)\left(a r-a^{2}+3 r-a+2\right), \\
& 2 s\left(5-3 r-a^{2}+a r\right)=(2 s-1)\left(5-6 r+2 r^{2}-a^{2}\right)+\left(5-a^{2}\right) .
\end{aligned}
$$


Transposing $i$ and $j$ above, the quantum Serre relation $[2] E_{j i j}=E_{j j i}+E_{i j j}$ gives equations:

$$
\begin{aligned}
& 2(1-r)\left(3 s-b+2-b^{2}+b s\right)=\left(5-b^{2}\right)+(1-2 r)\left(2 s^{2}+2 s+1-b^{2}\right), \\
& 2=r\left(5-3 s-b^{2}+b s\right)+(1-r)\left(b s-b^{2}+3 s-b+2\right), \\
& 2 r\left(5-3 s-b^{2}+b s\right)=(2 r-1)\left(5-6 s+2 s^{2}-b^{2}\right)+\left(5-b^{2}\right) .
\end{aligned}
$$

Solving equations (117), (118), one obtains the following two solutions:

$$
\left\{\begin{array} { l } 
{ a = b = 1 , } \\
{ r = s = 1 . }
\end{array} \quad \left\{\begin{array}{l}
a=b=-1, \\
r=s=0 .
\end{array}\right.\right.
$$

Notice that the two systems of parameters give rise to the differentials $\partial(1,1,1,1)$, $\partial(-1,-1,0,0)$ which are conjugate to each other under the (anti-)automorphisms $\psi$ and $\sigma$ (Proposition 4.6).

The above discussion shows that these two groups of special parameters are necessary for the quantum Serre relations to hold in $K_{0}\left(R\left(\underset{i}{\bullet} \rightarrow_{j}^{\bullet}\right), \partial(a, b, r, s)\right)$. Next we show that under these special parameters, we do have the equality of symbols

$$
[2]\left[P_{i j i}\right]=\left[P_{i i j}\right]+\left[P_{j i i}\right], \quad[2]\left[P_{j i j}\right]=\left[P_{j j i}\right]+\left[P_{i j j}\right],
$$

in the Grothendieck group. In fact, we will prove that the following divided power form of the quantum Serre relations

$$
\left[P_{i j i}\right]=\left[P_{i^{(2)} j}\right]+\left[P_{j i(2)}\right], \quad\left[P_{j i j}\right]=\left[P_{j^{(2)}}\right]+\left[P_{i j^{(2)}}\right],
$$

holds in $K_{0}\left(R\left(\bullet_{i} \rightarrow \underset{j}{\bullet}\right)\right)$ under the differentials $\partial(1,1,1,1)$ or $\partial(-1,-1,0,0)$. Since these two differentials are conjugate to each other, it suffices to do this for $\partial(1,1,1,1)$.

Lemma 4.10. Equipped with the differential $\partial(1,1,1,1)$, the module

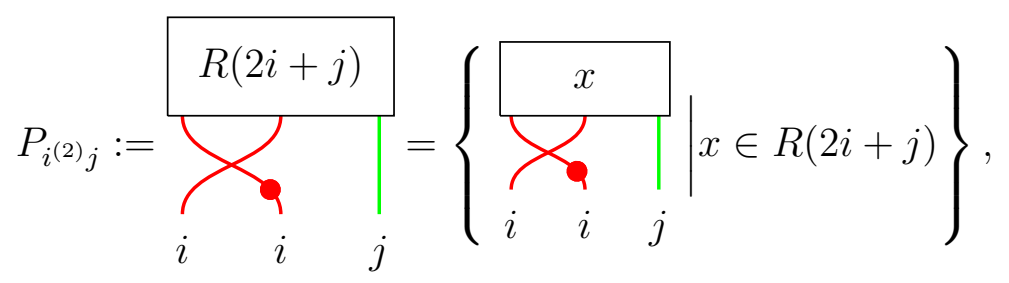

is compact cofibrant, and likewise for the module $P_{j i(2)}$.

Proof. As we have seen in Section 2, when $a=1$, the polynomial module $P_{2, i}:=\sum_{i}^{R(2 i)}$ with the differential $\partial_{1}$ is compact cofibrant (Proposition 3.26), and likewise for $P_{1, j} \cong$ $\mathbb{k}[x(j)]$. The module in the statement of the lemma is easily seen to be no other than $\operatorname{Ind}_{R(2 i) \otimes R(j)}^{R(2 i+j)}\left(P_{2, i} \otimes P_{1, j}\right)$. Hence the claim follows since Ind preserves compactness and cofibrance. 
Under $\partial(1,1,1,1)$, one computes easily that

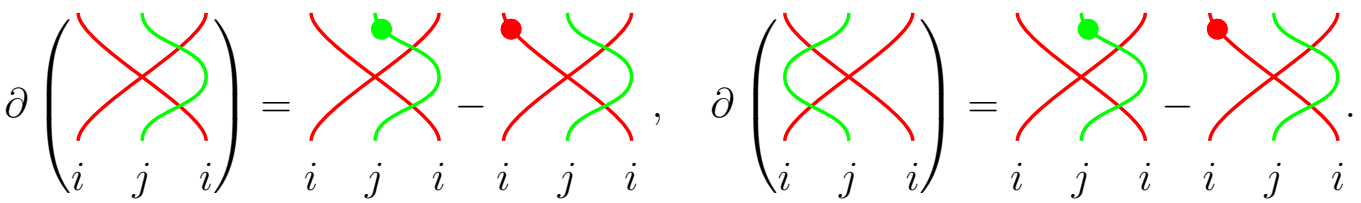

Therefore $P_{i j i}$ fits into the short exact sequence of $p$-DG modules

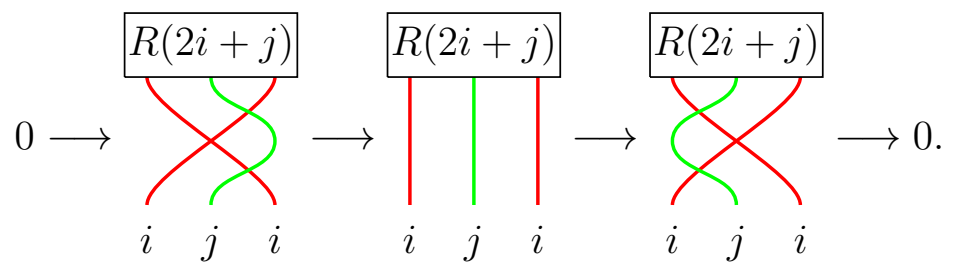

Here the differential acts on the generator of the quotient module trivially:
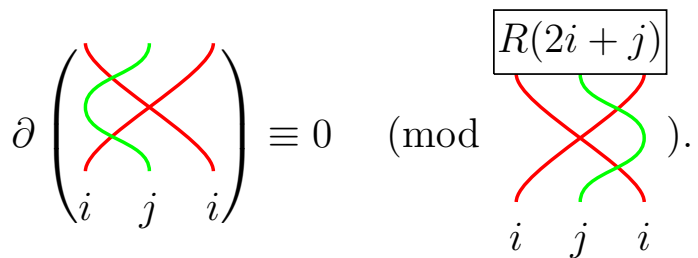

Now we claim that the isomorphisms of projective $R(2 i+j)$-modules defined in equations (114), (115) lift to isomorphisms of $p$-DG modules. Here the module

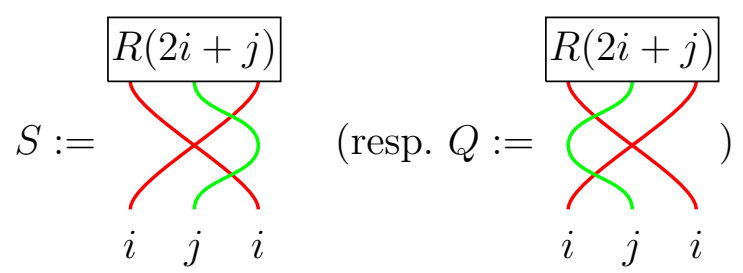

is equipped with the $p$-DG submodule structure (resp. quotient module structure) of $P_{i j i}$. The differential acts on the module generator $\overbrace{i}$ of $S$ by the first equation of (120), while $\partial$ acts as zero on $i \quad j \quad i$

intertwining relations $\rho_{i^{(2)} j} \circ \partial_{S}=\partial_{P_{i(2)}} \circ \rho_{i(2) j}$ on $S$, and $\rho_{j i(2)} \circ \partial_{Q}=\partial_{P_{j i(2)}} \circ \rho_{j i(2)}$ on the quotient module $Q$. See equations (112), (113) for the maps $\rho_{i(2)}, \rho_{j i(2)}, \vartheta_{i^{(2)} j}$ and $\vartheta_{j i(2)}$. Equivalently $\vartheta_{i(2) j} \circ \partial_{P_{i(2)}} \circ \rho_{i(2) j}=\partial_{S}$ and $\vartheta_{j i(2)} \circ \partial_{P_{j i}(2)} \circ \rho_{j i(2)}=\partial_{Q}$. 
Given any element $x$ of $R(2 i+j)$,

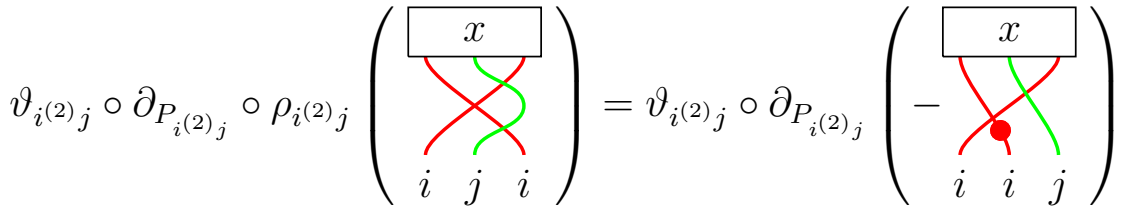

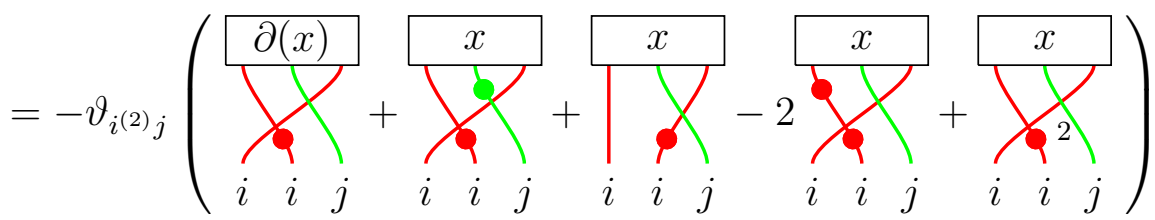

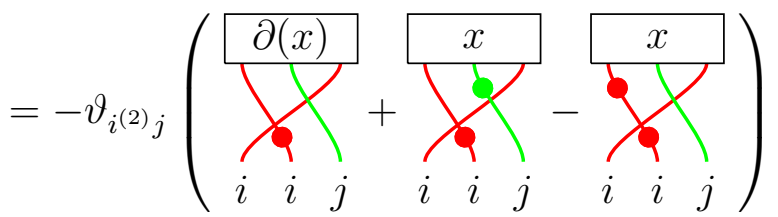

$$
\begin{aligned}
& =\sum_{i=i}^{\partial(x)}-\sum_{i=1}^{x}
\end{aligned}
$$

Comparing this with the first equation of (120) one obtains the claimed intertwining relation. Likewise, on the quotient module,

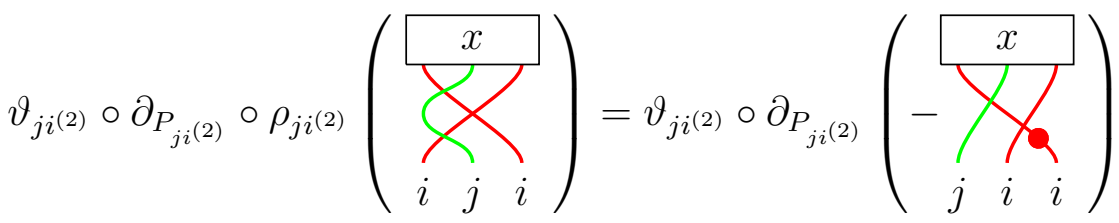

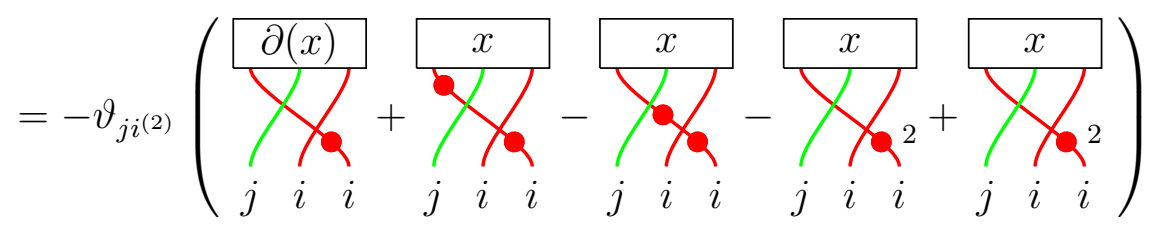

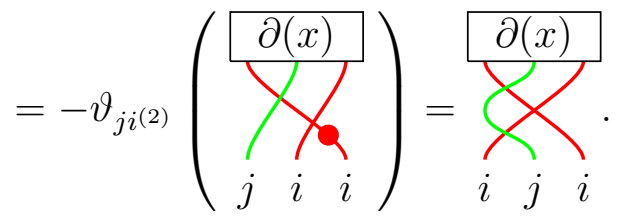

The case when $i, j$ are transposed is entirely similar and left to the reader. We summarize the above discussion into the following result. 
Proposition 4.11. The following equalities of symbols

$$
\left\{\begin{array}{l}
{[2]\left[P_{i j i}\right]=\left[P_{i i j}\right]+\left[P_{j i i}\right]} \\
{[2]\left[P_{j i j}\right]=\left[P_{j j i}\right]+\left[P_{i j j}\right]}
\end{array}\right.
$$

hold in the Grothendieck group $K_{0}\left(R\left(\bullet_{i} \rightarrow \underset{j}{\bullet}\right), \partial\left(a_{i}, a_{j}, r_{i j}, r_{j i}\right)\right)$ if and only if the parameters $\left(a_{i}, a_{j}, r_{i j}, r_{j i}\right)$ are given by either

$$
\left\{\begin{array} { l } 
{ a _ { i } = a _ { j } = 1 , } \\
{ r _ { i j } = r _ { j i } = 1 , }
\end{array} \quad \text { or } \quad \left\{\begin{array}{l}
a_{i}=a_{j}=-1, \\
r_{i j}=r_{j i}=0
\end{array}\right.\right.
$$

The discussion of this subsection gives in fact more than just the relations of symbols in the Grothendieck group. If $\partial$ is parameterized by the first system of coefficients, there are short exact sequences of $R(2 i+j)_{\partial}$-modules (resp. $R(2 j+i)_{\partial}$-modules):

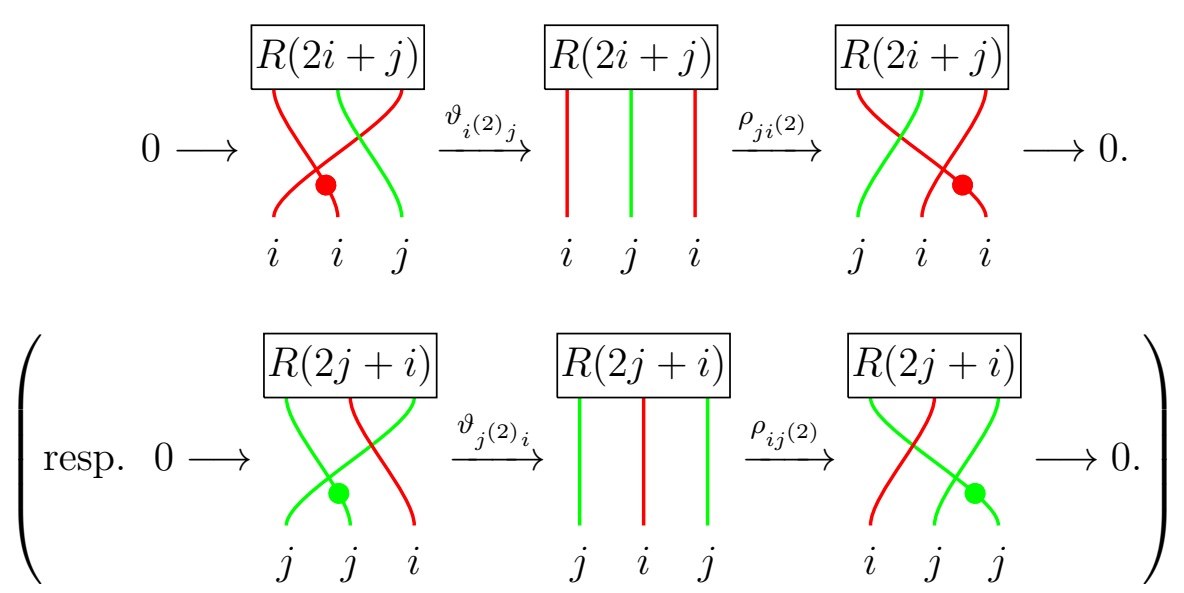

Likewise if $\partial$ is parameterized by the second system of equations, one obtains similar short exact sequences by applying the symmetry $\sigma$ (Proposition 4.6) to the above ones. The short exact sequences (122), (123) give rise to exact triangles in the homotopy category and derived category (Lemma 2.16 and 2.20$)$ :
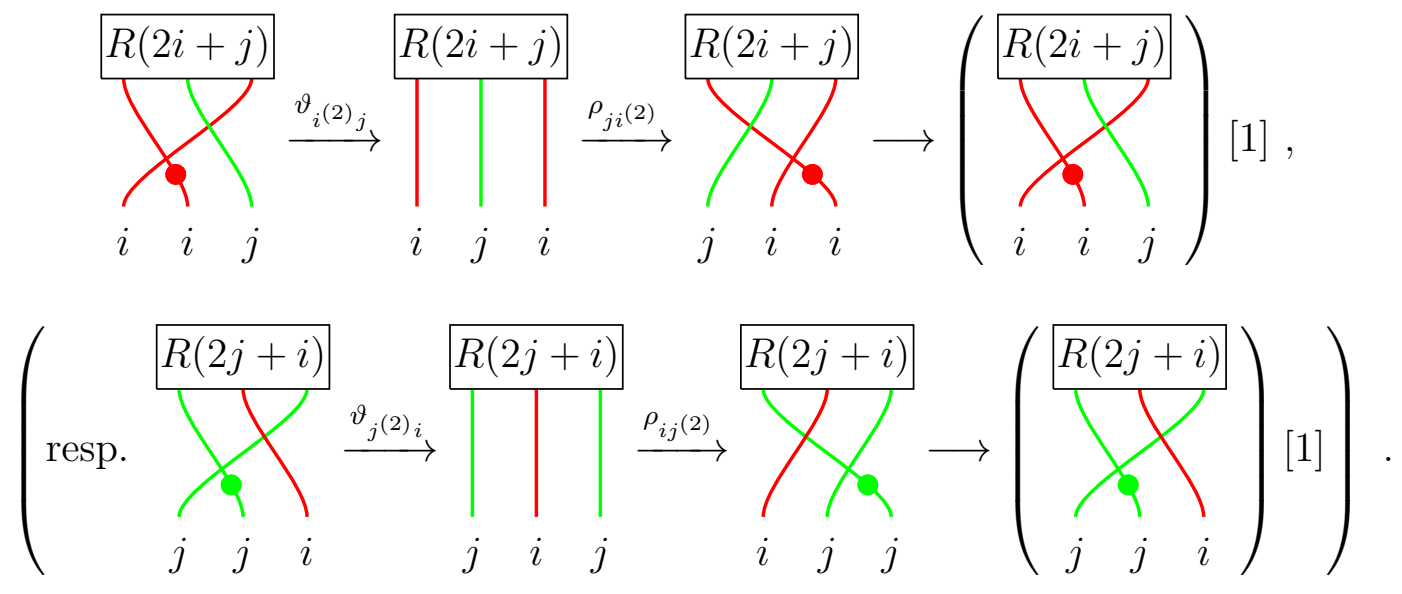
We regard the exact triangles (124) and (125) as categorical liftings of the divided power form of the quantum Serre relations

$$
E_{i j i}=E_{i(2)}+E_{j i(2)}, \quad\left(\operatorname{resp} E_{j i j}=E_{j^{(2)} i}+E_{i j(2)}\right) .
$$

We extend the results to general simply-laced Cartan data in what follows.

Remark 4.12 $\left(\mathfrak{s l}_{3}\right.$ at $\left.\sqrt{-1}\right)$. At a fourth root of unity $\zeta_{4}= \pm \sqrt{-1}$, the quantum Serre relations $[2] E_{i j i}=E_{i i j}+E_{j i i},[2] E_{j i j}=E_{j j i}+E_{i j j}$ for the small quantum group $u_{\zeta_{4}}^{+}\left(\mathfrak{s l}_{3}\right)$ are redundant, since in this case $[2]=0, E_{i}^{2}=0$, and $E_{j}^{2}=0$. By computing the endomorphism algebras of the $p$-DG modules as we did in Example 4.9, one finds that $P_{i i j}, P_{j i i}, P_{j j i}$ and $P_{i j j}$ are all contractible, while $P_{i j i}, P_{j i j}$ are not. Furthermore, one can also show that the symbols of $P_{j i j i}, P_{i j i j}, P_{i j i j i}$ etc. are not zero in the Grothendieck group of $\left(R\left(A_{2}\right), \partial(1,1,1,1)\right)$.

Idempotents and $p$-DG filtrations. Some of the manipulations on the previous few pages can be restated more intrinsically as follows. Let $R$ be a ring and $x, y \in R$ be two elements satisfying

$$
x y x=x, \quad y x y=y .
$$

Then $x y$ and $y x$ are idempotents in $R$ and the projective left $R$-modules $R x y$ and $R y x$ are isomorphic via the maps that take $z x y \in R x y$ to $z x y x=z x \in R y x$ and $z y x \in R y x$ to $z y x y=z y \in R x y$, respectively:

$$
R x y \stackrel{\cdot x}{\underset{y}{\longrightarrow}} R y x .
$$

Assume now that $R$ carries a $p$-nilpotent derivation $\partial$. The projective modules $R x y$ and $R y x$ are $\partial$-closed if and only if

$$
\partial(x y) \in R x y, \quad \partial(y x) \in R y x .
$$

The maps in (127) will commute with $\partial$ if and only if

$$
\partial(x y) x=\partial(x y x), \quad \partial(y x) y=\partial(y x y) .
$$

Expanding via the Leibniz rule, (129) is equivalent to

$$
x y \partial(x)=0, \quad y x \partial(y)=0 .
$$

The following conditions are equivalent to (128) and (130):

$$
x \partial(y)=0, \quad y \partial(x)=0 .
$$


Indeed, to see that (131) implies (128), one computes

$$
\partial(x y)=\partial(x) y+x \partial(y)=\partial(x) y=\partial(x) y x y \in R x y \text {. }
$$

Likewise, (131) gives $\partial(y x) \in R y x$.

Suppose that $R$ has, in addition, elements $x^{\prime}, y^{\prime}$ such that

$$
x^{\prime} y^{\prime} x^{\prime}=x^{\prime}, \quad y^{\prime} x^{\prime} y^{\prime}=y^{\prime}
$$

and

$$
y^{\prime} x=0, \quad y x^{\prime}=0 .
$$

Then $x^{\prime} y^{\prime}, y^{\prime} x^{\prime}$ are also idempotents in $R$, and the projective $R$-modules $R x^{\prime} y^{\prime}$ and $R y^{\prime} x^{\prime}$ are isomorphic. Furthermore, $x y$ and $x^{\prime} y^{\prime}$ are mutually orthogonal, $e:=x y+x^{\prime} y^{\prime}$ is an idempotent, and there is an isomorphism of projective left $R$-modules

$$
R e \cong R x y \oplus R x^{\prime} y^{\prime} \cong R y x \oplus R y^{\prime} x^{\prime},
$$

which can be presented via the diagram

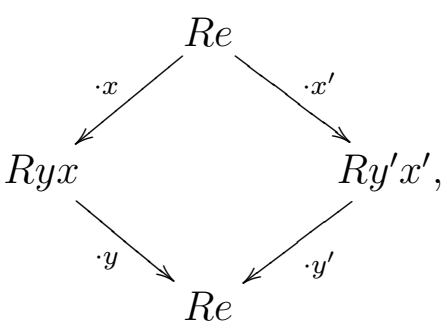

with $\cdot x$ given by right multiplication by $x$ which takes ze to $z e x \in R y x$, etc.

It is, in general, too much to hope that $\partial$ would respect this direct sum decomposition of $R e$. Instead, we would like $R x y \subset R e$ to be a $p$-DG submodule isomorphic to $R y x$, and the quotient $R e / R x y$ to be $p$-DG isomorphic to $R y^{\prime} x^{\prime}$. Therefore, in addition to (128), we want the maps

$$
R e / R x y \stackrel{\cdot x^{\prime}}{\underset{: y^{\prime}}{\longrightarrow}} R y^{\prime} x^{\prime} .
$$

to commute with the $p$-differentials. This is equivalent to the condition that $R y^{\prime} x^{\prime}$ is $\partial$-closed

$$
\partial\left(y^{\prime} x^{\prime}\right) \in R y^{\prime} x^{\prime}
$$

and that

$$
\partial(e) x^{\prime}=\partial\left(e x^{\prime}\right), \quad \partial\left(y^{\prime} x^{\prime}\right) y^{\prime} \equiv \partial\left(y^{\prime} x^{\prime} y^{\prime}\right)(\bmod R x y)
$$

A simple computation shows that condition (135) is implied by (136), the latter in turn being equivalent to the conditions

$$
y^{\prime} \partial\left(x^{\prime}\right)=0, \quad x^{\prime} \partial\left(y^{\prime}\right) \in R x y .
$$


Furthermore, Re is $\partial$-closed when (137) is satisfied. Then, there is an exact sequence of $R_{\partial}$-modules

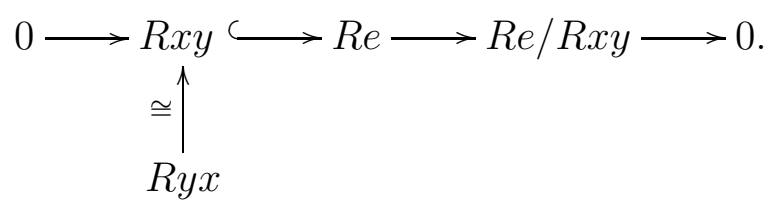

The surjective map $R e \longrightarrow R y^{\prime} x^{\prime}$ given by right multiplication by $x^{\prime}$ has $R x y$ as its kernel and commutes with $\partial$ since $y^{\prime} \partial\left(x^{\prime}\right)=0$. Consequently, there is a short exact sequence of $(R, \partial)$-modules (equivalently, a $p$-DG filtration on $R e$ )

$$
0 \longrightarrow R y x \stackrel{\cdot y}{\longrightarrow} R e \stackrel{\cdot x^{\prime}}{\longrightarrow} R y^{\prime} x^{\prime} \longrightarrow 0 .
$$

If $R y x$ and $R y^{\prime} x^{\prime}$ are compact, then so is $R e$ and this filtration leads to a relation in $K_{0}(R, \partial)$

$$
[R e]=[R y x]+\left[R y^{\prime} x^{\prime}\right]
$$

In the case of $R=R(2 i+j)$ with the differential $\partial(1,1,1,1)$, let

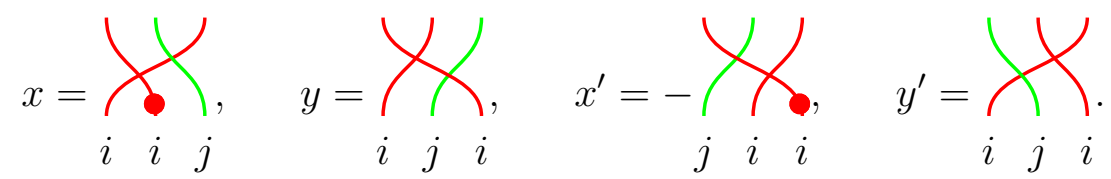

An easy computation shows that conditions (126), (131), (132), (133), (137) hold. Idempotents $y x, y^{\prime} x^{\prime}$, and $e$ give rise to compact $R(2 i+j)_{\partial}$-modules $P_{i(2)}, P_{j i(2)}$ (Lemma 4.10), and $P_{i j i}$ respectively, resulting in the short exact sequence

$$
0 \longrightarrow P_{i(2)}{ } \longrightarrow P_{i j i} \longrightarrow P_{j i(2)} \longrightarrow 0
$$

and in the relation in the Grothendieck group

$$
\left[P_{i j i}\right]=\left[P_{i(2)}\right]+\left[P_{j i(2)}\right] .
$$

The general case. In general, let $\Gamma$ be a connected simply-laced Cartan datum with vertex set $I$ and an arbitrary orientation. Define $(R(\Gamma), \partial)$ to be the associated $p$-DG algebra with the differential parameter chosen as in equations (97) through (102). For any sequence of colors $\mathbf{i}=i_{1} i_{2} \cdots i_{n} \in I^{n}$ such that $\sum_{t=1}^{n} i_{t}=\nu \in \mathbb{N}[I]$, define the compact cofibrant $p$-DG module over $(R(\Gamma), \partial)$ by

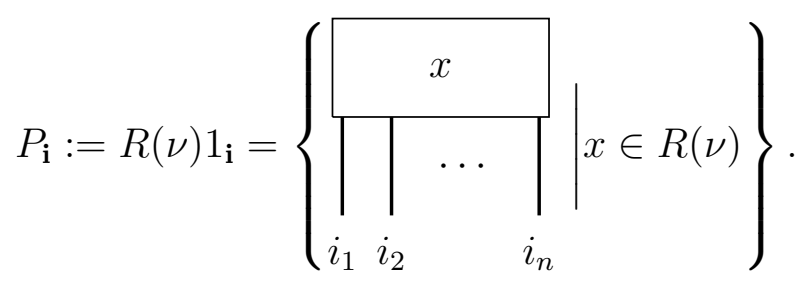

The local nature of the $p$-DG algebra together with the rank-two cases (Propositions 4.8 and 4.11) applied to each pair of vertices in $\Gamma$ leads to the following. 
(I) If $i, k$ are distant vertices in $\Gamma$, and $u_{i k}=u_{k i}=0$, then there is an isomorphism of left $(R(\Gamma), \partial)$-modules

$$
\eta_{k i}: P_{\ldots i k \ldots} \longrightarrow P_{\ldots k i \ldots}
$$

where $\eta_{k i}$ is given by post-composing any element in $P_{\ldots i k \cdots}$ with

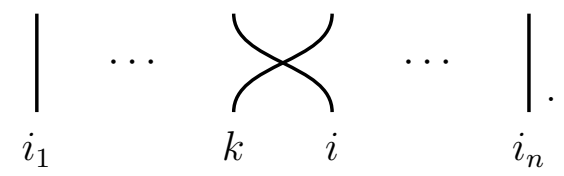

The inverse map $\eta_{i k}$ of $\eta_{k i}$ is given by attaching to any element of $P_{\ldots k i \ldots}$ from below the element

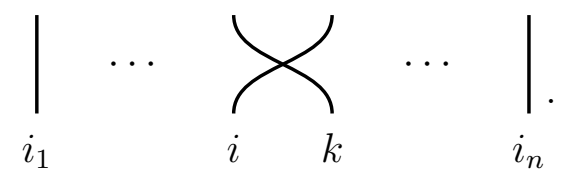

(II) If $i, j$ are vertices in $\Gamma$ connected by the oriented edge $i \rightarrow j$, and the parameters satisfy

$$
a_{i}=a_{j}=r_{i j}=r_{j i}=1,
$$

then there are short exact sequences of left $R(\Gamma)_{\partial}$-modules

$$
\begin{gathered}
0 \longrightarrow P_{\ldots i(2) j \ldots} \stackrel{\vartheta_{i^{(2)} j}}{\longrightarrow} P_{\ldots i j i \ldots} \stackrel{\rho_{j i}(2)}{\longrightarrow} P_{\ldots j i(2) \ldots} \longrightarrow 0, \\
0 \longrightarrow P_{\ldots j(2) i \ldots} \stackrel{\vartheta_{j(2)}}{\longrightarrow} P_{\ldots j i j \ldots} \stackrel{\rho_{i j}(2)}{\longrightarrow} P_{\ldots i j(2) \ldots} \longrightarrow 0 .
\end{gathered}
$$

Here $\vartheta_{i^{(2)} j}, \rho_{j i^{(2)}}, \vartheta_{j^{(2)} i}$ and $\rho_{i j^{(2)}}$ are respectively right multiplication by the elements

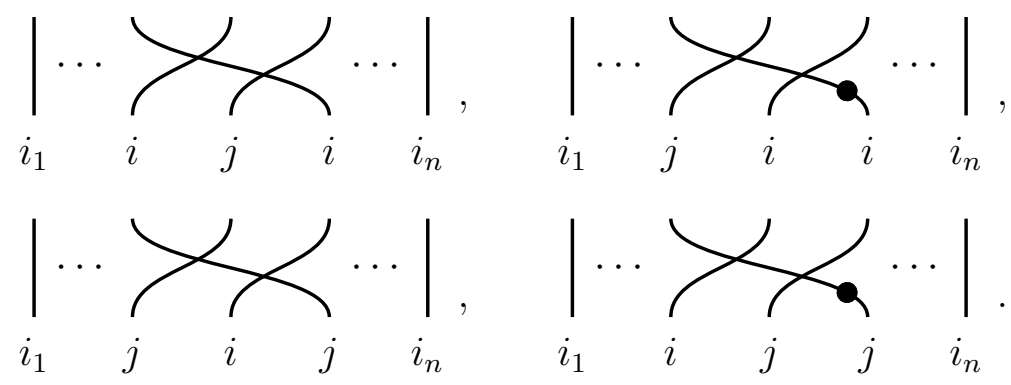

$\left(I I^{\sigma}\right)$ If $i, j$ are vertices in $\Gamma$ connected by the oriented edge $i \rightarrow j$, and the parameters satisfy

$$
1+a_{i}=1+a_{j}=r_{i j}=r_{j i}=0,
$$

then there are short exact sequences of left $R(\Gamma)_{\partial}$-modules

$$
0 \longrightarrow P_{\ldots j i(2) \ldots} \stackrel{\vartheta_{j i}(2)}{\longrightarrow} P_{\ldots i j i \ldots} \stackrel{\rho_{i(2)}}{\longrightarrow} P_{\ldots i(2)} j \ldots \longrightarrow 0
$$




$$
0 \longrightarrow P_{\ldots i j(2) \ldots} \stackrel{\vartheta_{i j}(2)}{\longrightarrow} P_{\ldots j i j \ldots} \stackrel{\rho_{j^{(2)}}}{\longrightarrow} P_{\ldots j}(2) i \ldots \longrightarrow 0
$$

where $\vartheta_{j i(2)}, \rho_{i(2)}, \vartheta_{i j(2)}$ and $\rho_{j(2)}$ are respectively given by right multiplication with the elements

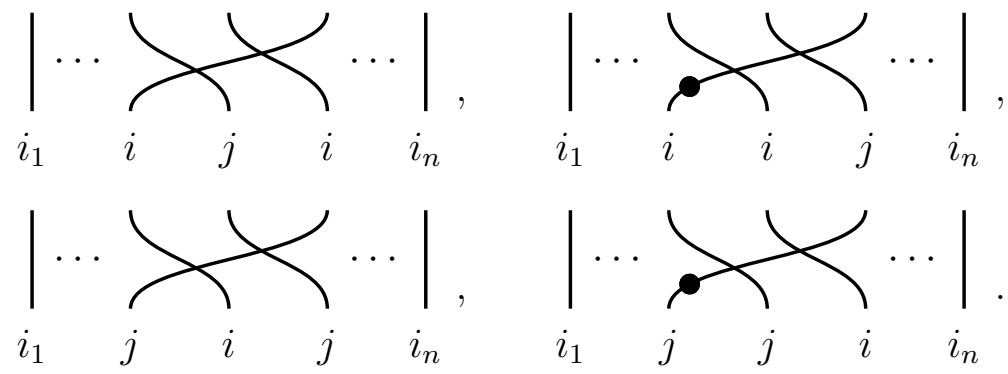

$\left(I I^{\sigma}\right)$ follows from $(I I)$ by applying the symmetry $\sigma$ (see equation (94)) and using Proposition 4.6. We leave the verification of these statements to the reader. The following definition summarizes the two parameters of differentials for which the categorical quantum Serre relations hold.

Definition 4.13. Let $\Gamma$ be a connected simply-laced Cartan datum with the set of vertices $I$ and an arbitrary orientation, and $R(\Gamma)$ be its associated KLR algebra.

$\left(D_{+}\right)$The $p$-nilpotent local differential $\partial_{1}$ on $R(\Gamma)$ acts on the one-strand generators by

$$
\partial_{1}\left(\begin{array}{l}
\downarrow \\
i
\end{array}\right)=\oint_{i}^{2},
$$

while on the two-strand generators,

$$
\begin{aligned}
& \partial_{1}\left(\succ_{i_{1}}^{\zeta}\right)=\left.\left.\delta_{i_{1}, i_{2}}\right|_{i_{1}}\right|_{i_{2}}-i_{1} \cdot i_{2} \overbrace{i_{1}} \\
& \left\{\begin{array}{l}
\left.\left.\right|_{i_{1}} ^{\mid}\right|_{i_{2}} ^{\mid} \\
\sum_{i_{1}} \sum_{i_{2}} \\
\text { if } i_{1}, i_{2} \text { are distant, }
\end{array}\right.
\end{aligned}
$$

Here $i, i_{1}, i_{2} \in I$ are vertices of $\Gamma$, and $i_{1} \cdot i_{2}$ is the Cartan pairing between $i_{1}, i_{2}$. 
$\left(D_{-}\right)$The $p$-nilpotent local differential $\partial_{-1}$ on $R(\Gamma)$ acts on the one-strand generators by

$$
\partial_{-1}\left(\begin{array}{l}
\downarrow \\
i
\end{array}\right)=\varliminf_{i}^{2},
$$

while on the two-strand generators,

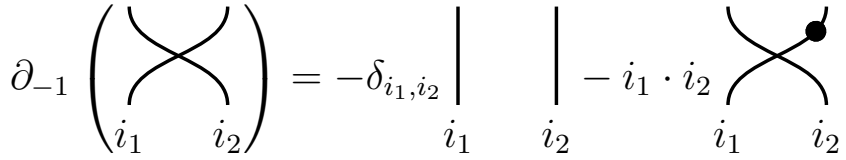

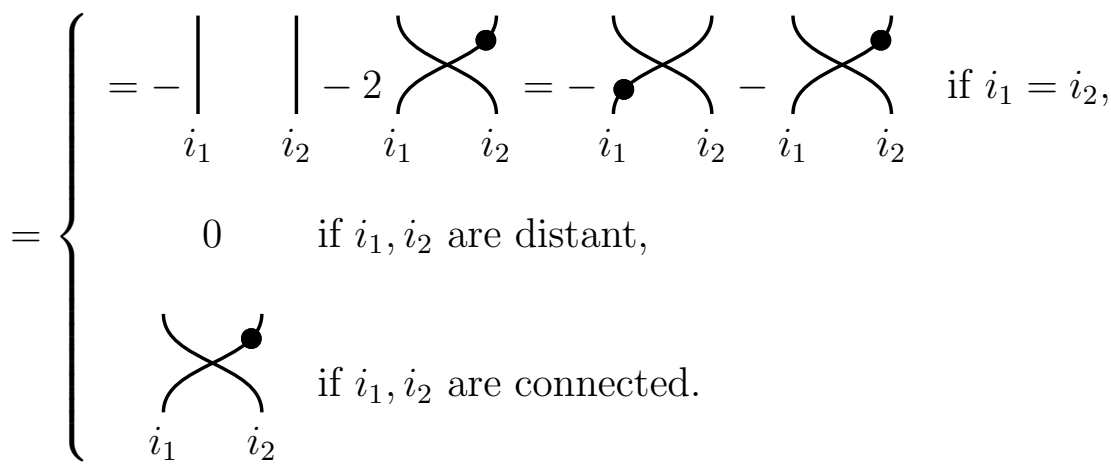

The next theorem summarizes the discussion in the subsection.

Theorem 4.14. The relations

$$
\left[P_{\ldots i k \cdots}\right]=\left[P_{\ldots k i \cdots}\right]
$$

where $i, k$ are distant vertices, and

$$
[2]\left[P_{\ldots i j i \ldots]}\right]=\left[P_{\ldots i i j \ldots]}\right]+\left[P_{\ldots j i i \ldots}\right],
$$

where $i, j$ are vertices connected by one edge in $\Gamma$ with any orientation, hold in the Grothendieck group $K_{0}(R(\Gamma), \partial)$ if and only if $\partial=\partial_{ \pm 1}$. The differentials $\partial_{ \pm 1}$ are conjugate to each other by the (anti-)automorphisms $\psi$ and $\sigma$ of $R(\Gamma)$. Furthermore, the following statements hold.

(i) In the derived category $\mathcal{D}\left(R(\Gamma), \partial_{1}\right)$, there is an isomorphism of $p$-DG modules

$$
P_{\ldots i k \cdots} \cong P_{\ldots k i \cdots}
$$

if $i, k$ are distant vertices. There is an exact triangle if $i$ and $j$ are vertices connected by one edge

$$
P_{\ldots i(2) j \ldots} \longrightarrow P_{\ldots i j i \ldots} \longrightarrow P_{\ldots j i(2) \ldots} \longrightarrow P_{\ldots i(2)} \ldots[1] .
$$


(ii) In the derived category $\mathcal{D}\left(R(\Gamma), \partial_{-1}\right)$, there is an isomorphism of $p$-D $G$ modules

$$
P_{\ldots i k \cdots} \cong P_{\ldots k i \cdots}
$$

if $i, k$ are distant vertices, while there is an exact triangle

$$
P_{\ldots j i(2) \ldots} \longrightarrow P_{\ldots i j i \ldots} \longrightarrow P_{\ldots i(2) j \ldots} \longrightarrow P_{\ldots j i(2) \ldots[1]}
$$

if $i$ and $j$ are connected by one edge.

\subsection{Grothendieck groups for small weights}

In this subsection we compute Grothendieck groups of $p$-DG module categories over $(R(\nu), \partial)$ for certain small weights $\nu$ in rank two. From now on we will specialize the definition of $\partial$ to the first system of parameters $\left(D_{+}\right)$in Definition 4.13,

Example 4.15. Let $i, k$ be distant vertices. The $p$-DG algebra $R(i+k)$ has a basis consisting of elements

$$
\left\{\left.\left.\right|_{i} ^{n_{1}}\right|_{k} ^{n_{2}},\left.\left.\right|_{k} ^{n_{1}}\right|_{i} ^{n_{2}}, \sum_{i}^{n_{1}} \sum_{k}^{n_{2}}, \sum_{k}^{b_{1}^{n_{1}} \boldsymbol{\phi}^{n_{2}} \mid} \mid n_{1}, n_{2} \in \mathbb{N}\right\},
$$

and the differential $\partial$ acts trivially on diagrams without dots. The inclusion of the $2 \times 2$ matrix algebra with trivial differential

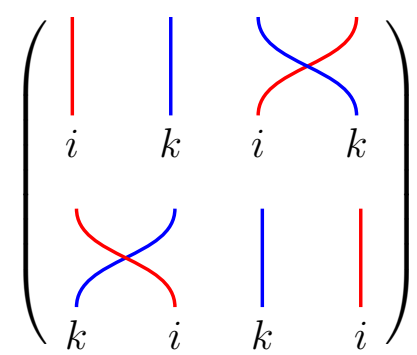

into $R(i+k)$ is a quasi-isomorphism of $p$-DG algebras. By Theorem 2.37, this inclusion induces an equivalence of derived categories, and hence Grothendieck groups. From the toy matrix model in Section 2.3, one concludes that $K_{0}(R(i+k)) \cong \mathbb{O}_{p}$.

This example generalizes to any weight space of $A_{1} \times A_{1}$, using Theorem 3.35, One concludes that the Grothendieck group of the $p$-DG algebra $\left(R\left(\begin{array}{ll}\bullet & \bullet \\ i\end{array}\right), \partial\right)$ can be identified with the twisted bialgebra

$$
K_{0}(R(\stackrel{\bullet}{\bullet} \quad \stackrel{\bullet}{\bullet}), \partial) \cong u_{\mathbb{O}_{p}}^{+}\left(\mathfrak{s l}_{2} \times \mathfrak{s l}_{2}\right) .
$$

In what follows we will focus on the $A_{2}$ case $\underset{i}{\bullet} \rightarrow \underset{j}{\bullet}$. 
Example 4.16. The $\mathbb{k}$-algebra $R(i+j)$ has a basis

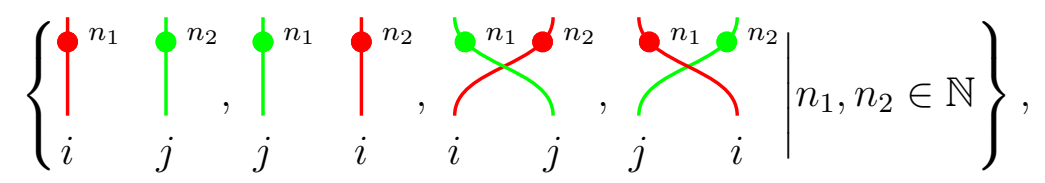

and the differential $\partial$ acts on the generators by

$$
\begin{aligned}
& \partial\left(\mid \begin{array}{ll}
\mid & \mid \\
i & j
\end{array}\right)=0, \quad \partial\left(\begin{array}{ll} 
& \\
j & i
\end{array}\right)=0 \\
& \partial\left(\zeta_{i} \zeta_{j}\right)=\sum_{i}^{k}, \partial\left(\zeta_{j}\right)=\sum_{j}^{k}
\end{aligned}
$$

Consider the two-sided ideal

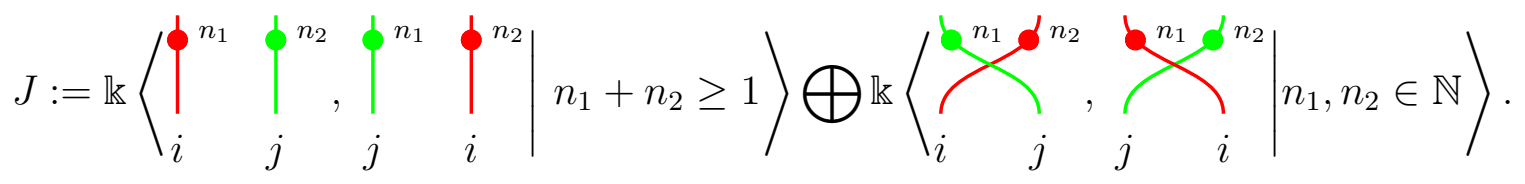

It is preserved by the differential and forms a contractible $p$-complex. Therefore, the quotient $p$-DG algebra $(R(i+j) / J, \partial)$ is isomorphic to

$$
\begin{array}{r|l|l|}
\mathbb{k} \mid & |\times \mathbb{k}| & \cong \mathbb{k} \times \mathbb{k} \\
i & j \quad j \quad i
\end{array}
$$

with trivial differential, and the natural projection $\pi: R(i+j) \longrightarrow R(i+j) / J$ is a quasi-isomorphism. Alternatively, the inclusion of the algebras $\mathbb{k} \times \mathbb{k} \hookrightarrow R(i+j)$ is a quasi-isomorphism of $p$-DG algebras. By Theorem 2.37, there is an equivalence of triangulated categories $\mathcal{D}(R(i+j)) \cong \mathcal{D}(\mathbb{k} \times \mathbb{k})$, implying that $K_{0}(R(i+j)) \cong \mathbb{O}_{p} \oplus \mathbb{O}_{p}$.

To compute the next example we will use the following observation. Let $(R, \partial)$ be a $p$-DG algebra and $\epsilon_{1}, \epsilon_{2} \in R$ be idempotents such that $R \epsilon_{t}$ is a $p$-DG submodule of $R$ for $t=1,2$. Necessarily $\partial\left(\epsilon_{t}\right)=r_{t} \epsilon_{t}$ for some $r_{t} \in R$. Then under the isomorphism of $\mathbb{k}$-spaces $\operatorname{HOM}_{R}\left(R \epsilon_{1}, R \epsilon_{2}\right) \cong \epsilon_{1} R \epsilon_{2}$, the natural $\partial$-action on the HOM-space translates into an action on $\epsilon_{1} R \epsilon_{2}$, denoted by " $\diamond$ ", as follows. For any $x \in R$,

$$
\partial \diamond\left(\epsilon_{1} x \epsilon_{2}\right)=\epsilon_{1} \cdot \partial\left(x \epsilon_{2}\right)
$$

Indeed, if $f \in \mathrm{HOM}_{R}\left(R \epsilon_{1}, R \epsilon_{2}\right)$, then $\partial(f)\left(y \epsilon_{1}\right)=\partial\left(f\left(y \epsilon_{1}\right)\right)-f\left(\partial\left(y \epsilon_{1}\right)\right)$ for any $y \in R$. Now if $f$ is a morphism defined by an element $\epsilon_{1} x \epsilon_{2}, f\left(y \epsilon_{1}\right)=y \epsilon_{1} x \epsilon_{2}$, then $\partial(f)\left(y \epsilon_{1}\right)=$ $\left.\partial\left(y \epsilon_{1} x \epsilon_{2}\right)-\partial\left(y \epsilon_{1}\right) \epsilon_{1} x \epsilon_{2}\right)=y \epsilon_{1}\left(\partial\left(x \epsilon_{2}\right)\right)$ and the claim follows. 
Example 4.17. We now compute the Grothendieck group of the $p$-DG algebra $(R(2 i+$ $j), \partial)$, assuming char $(\mathbb{k}) \geq 3$. By Proposition 2.28 , the triangulated category $\mathcal{D}(R(2 i+$ $j)$ ) is generated by the $p$-DG module $R(2 i+j)$, which is isomorphic to the direct sum of three compact cofibrant modules $P_{i i j}, P_{i j i}$ and $P_{j i i}$. Furthermore, $P_{i i j}$ has a two step filtration induced from the filtration (78) of $\mathrm{NH}_{2}$ by its polynomial representation. The subquotients of the filtration are isomorphic to grading shifts of $P_{i(2)}$. Likewise $P_{j i i}$ is filtered by grading shifts of $P_{j i(2)}$. The short exact sequence (121) and the $p$-DG enhanced isomorphisms (114), (115) show that $P_{i j i}$ fits into an exact triangle

$$
P_{i(2)} \longrightarrow P_{i j i} \longrightarrow P_{j i(2)} \longrightarrow P_{i^{(2)} j}[1] .
$$

Hence the modules $P_{i^{(2)} j}, P_{j i^{(2)}}$ are compact cofibrant generators of $\mathcal{D}(R(2 i+j))$, and Proposition 2.34 shows that there is an equivalence between $\mathcal{D}(R(2 i+j))$ and $\mathcal{D}\left(\left(\operatorname{END}_{R(2 i+j)}\left(P_{i^{(2)} j} \oplus P_{j i^{(2)}}\right)^{o p}\right)\right.$. We compute the differential on this endomorphism algebra using the remarks made before this example.

First off, since

$$
\operatorname{HOM}\left(P_{i i j}, P_{i i j}\right)=\mathbb{k}\left\langle\left.\left.\left.\right|_{i} ^{n_{1}}\right|_{i} ^{n_{2}}\right|_{j} ^{n_{3}} \sum_{i}^{n_{1}} \oint_{j}^{n_{2}} \phi^{n_{3}} \mid n_{1}, n_{2}, n_{3} \in \mathbb{N}\right\rangle,
$$

the endomorphism space of $P_{i^{(2)} j}$ is spanned by

$$
\operatorname{HOM}\left(P_{i^{(2)} j}, P_{i^{(2)} j}\right) \cong \mathbb{k}\left\langle\left.\left.\left.\left.\sum_{i}^{n_{1}}\right|_{j} ^{n_{2}}\right|_{i} ^{n_{i}}\right|_{j} ^{n_{3}}\right|_{n_{1}} ^{n_{2}} n_{2}, n_{3} \in \mathbb{N}\right\rangle .
$$

Terms in the second diagram vanish because there is a double crossing between the $i-i$ strands. To simplify terms in of the first diagram, one uses that two-variable polynomials form a rank-two module over $\mathrm{Sym}_{2}$ :

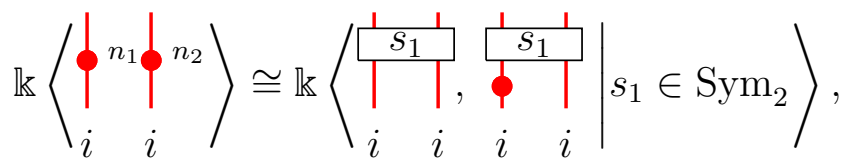

and therefore

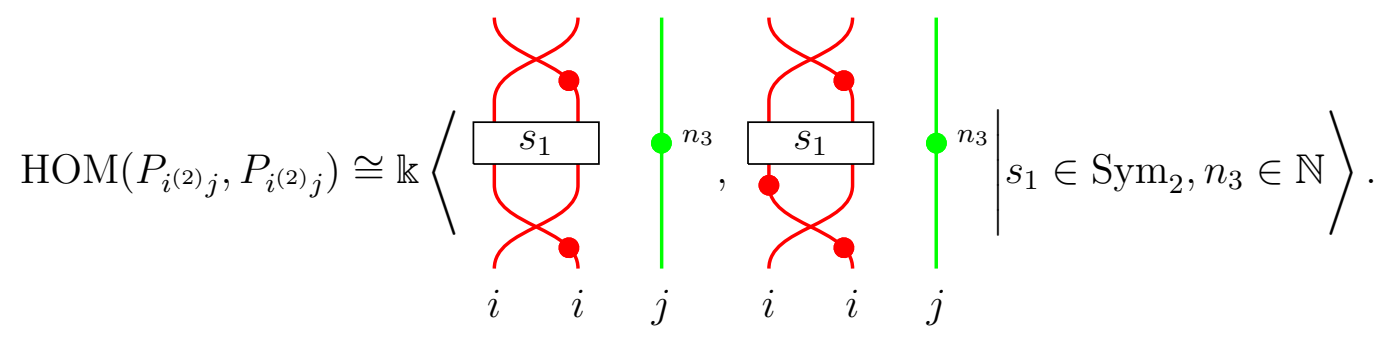


Using that symmetric polynomials can slide through crossings without obstruction, the HOM-space simplifies to be

$$
\begin{aligned}
\operatorname{HOM}\left(P_{i^{(2)} j}, P_{i(2)}\right) & \cong \mathbb{k}\left\langle\left.\right|_{k_{1}} \mid s_{1} \in \operatorname{Sym}_{2}\left[x_{1}(i), x_{2}(i)\right], k_{1} \in \mathbb{N}\right\rangle \\
& \left.\simeq \operatorname{Sym}_{2}\left[x_{1}(i), x_{2}(i)\right] \otimes \mathbb{k}\left[x_{3}(j)\right] \cdot\right|_{i} \mid
\end{aligned}
$$

On the module generator $\partial$ acts by

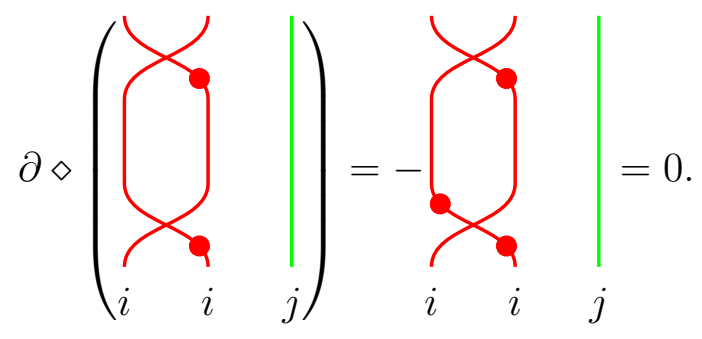

Hence the $p$-DG endomorphism algebra $\operatorname{HOM}\left(P_{i^{(2)} j}, P_{i^{(2)} j}\right)$ is isomorphic to the $p$ DG algebra $\operatorname{Sym}_{2}\left[x_{1}(i), x_{2}(i)\right] \otimes \mathbb{k}\left[x_{3}(j)\right]$ with the usual differential, which in turn is quasi-isomorphic to the ground field $\mathbb{k}$. Likewise, a similar computation shows that $\operatorname{HOM}\left(P_{j i(2)}, P_{j i(2)}\right)$ is also quasi-isomorphic to the ground field as a $p$-DG algebra.

Next, we compute the $\partial$ action on the space $\operatorname{HOM}\left(P_{j i^{(2)}}, P_{i^{(2)} j}\right)$. As in the previous case, one shows that the HOM-space is spanned by

$$
\operatorname{HOM}\left(P_{j i(2)}, P_{i^{(2)} j}\right) \cong{ }_{k}
$$


and $\partial$ acts on the generator by

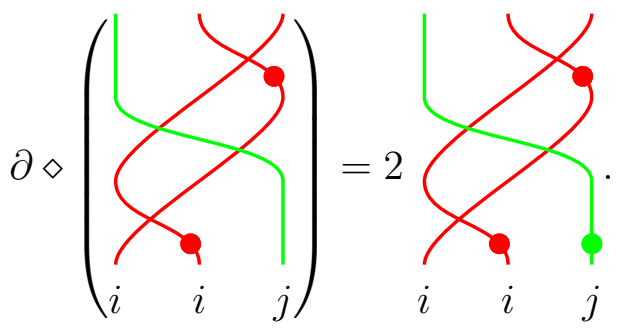

Thus, as a $p$-DG module over $\operatorname{Sym}_{2}\left[x_{1}(i), x_{2}(i)\right] \otimes \mathbb{k}\left[x_{3}(i)\right], \operatorname{HOM}\left(P_{j i(2)}, P_{i^{(2)} j}\right)$ is isomorphic to the left submodule (ideal) generated by $1 \otimes x_{3}^{2}(j)$ inside $\operatorname{Sym}_{2}\left[x_{1}(i), x_{2}(i)\right] \otimes$ $\mathbb{k}\left[x_{3}(i)\right]$. Therefore it is quasi-isomorphic to the $p$-complex

$$
\mathbb{k} \otimes\left(x_{3}^{2}(j) \longrightarrow x_{3}^{3}(j) \longrightarrow \cdots \longrightarrow x_{3}^{p}(j)\right) \cong V_{2}\{2\}
$$

since $\mathrm{Sym}_{2}$ is quasi-isomorphic to $\mathbb{k}$.

Lastly, we compute the $\operatorname{space} \operatorname{HOM}\left(P_{i^{(2)} j}, P_{j i(2)}\right)$ with the induced $\partial$-action.

$$
\operatorname{HOM}\left(P_{i(2) j}, P_{j i(2)}\right) \cong \mathbb{R}\left\langle s_{1} \in \operatorname{Sym}_{2}\left[x_{2}(i), x_{3}(i)\right], k_{1} \in \mathbb{N}\right\rangle .
$$

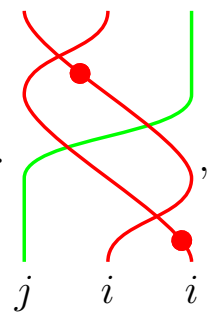

while $\partial$ acts on the module generator by

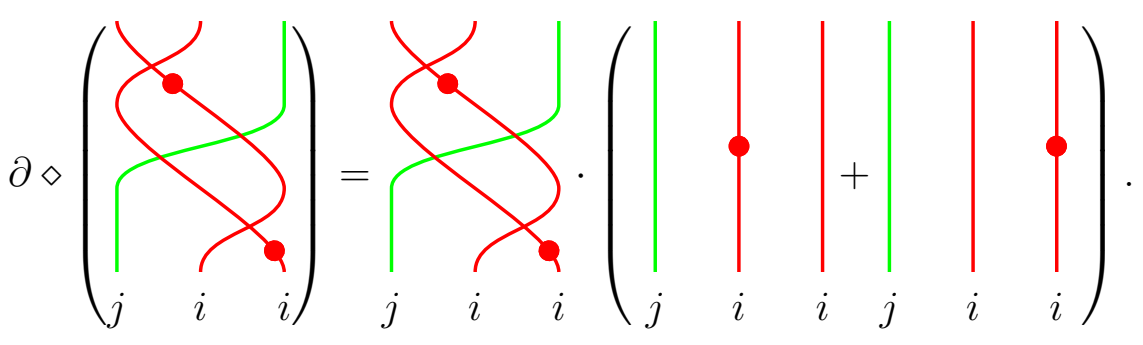

As a $p$-DG module over $\mathbb{k}\left[x_{1}(j)\right] \otimes \operatorname{Sym}_{2}\left[x_{2}(i), x_{3}(i)\right], \operatorname{HOM}\left(P_{i^{(2)} j}, P_{j i(2)}\right)$ is isomorphic to the left submodule in $\mathbb{k}\left[x_{1}(j)\right] \otimes \operatorname{Sym}_{2}\left[x_{2}(i), x_{3}(i)\right]$ generated by the element $1 \otimes$ $e_{2}\left(x_{2}(i), x_{3}(i)\right)=1 \otimes\left(x_{2}(i) x_{3}(i)\right)$. We now show that this submodule is contractible. 
Indeed it suffices to check that, under the usual differential on $\mathrm{Sym}_{2}, e_{2}=x_{1} x_{2} \in \mathrm{Sym}_{2}$ generates a contractible submodule. Note that $e_{2} \mathrm{Sym}_{2}$ fits into a short exact sequence

$$
0 \longrightarrow e_{2} \mathrm{Sym}_{2} \longrightarrow \mathrm{Sym}_{2} \longrightarrow \mathrm{Sym}_{1} \longrightarrow 0
$$

where $\operatorname{Sym}_{1} \cong \mathbb{k}\left[e_{1}\right]$ has the quotient differential structure $\partial\left(e_{1}\right)=e_{1}^{2}$. Therefore the surjection $\mathrm{Sym}_{2} \rightarrow \mathrm{Sym}_{1}$ of $p$-DG algebras, both quasi-isomorphic to the ground field, is a quasi-isomorphism. The contractibility of the kernel follows.

Given the above computation, it is more convenient to study the endomorphism algebra of the cofibrant module $\left(P_{i^{(2)} j} \otimes \widetilde{V}_{p-2}\{-p\}\right) \oplus P_{j i(2)} \cong P_{i^{(2)} j}[1] \oplus P_{j i(2)}$, which after all is also a compact generator. Combining the previous observations, we see that the endomorphism algebra is quasi-isomorphic to the quiver algebra with the trivial differential

$$
\operatorname{HOM}\left(P_{i^{(2)} j}[1] \oplus P_{j i^{(2)}}, P_{i^{(2)} j}[1] \oplus P_{j i(2)}\right) \cong \mathbb{k}(\bullet \longrightarrow \bullet)
$$

Here the arrow has degree one and the dots stands for the (quasi-isomorphic) onedimensional endomorphism spaces spanned by $\operatorname{Id}_{P_{i(2)}[1]}$ and $\operatorname{Id}_{P_{j i}(2)}$ respectively. By Proposition 2.34, we conclude that $\mathcal{D}(R(2 i+j)) \cong \mathcal{D}(\mathbb{k}(\bullet \longrightarrow \bullet))$. It follows from Proposition 2.39 that $K_{0}(R(2 i+j)) \cong \mathbb{O}_{p} \oplus \mathbb{O}_{p}$.

Further remarks. Let $\Gamma$ be a connected simply-laced Cartan datum. So far we have seen that, under the differentials given by the special parameters of Definition 4.13, shadows of the small quantum groups appear on the categorified level: relations $E_{i}^{p}=0$ are categorified into the triviality of the derived categories at weights pi (Proposition 3.15), quantum Serre relations hold (Theorem 4.14), and some direct comparison with the weight spaces in the simplest cases is shown in the above examples. We are tempted to propose the following conjecture.

Conjecture 4.18. Let $\Gamma$ be a Cartan datum of ADE type. With the parameters of the differential $\partial$ specified as in Definition 4.13, the $p-D G$ algebra $(R(\Gamma), \partial)$ categorifies an $\mathbb{O}_{p}$-integral form of the positive half of the small quantum group associated with $\Gamma$.

This conjecture is vague, as we do not describe a particular integral form of the quantum group over the ring $\mathbb{O}_{p}$ which $(R(\Gamma), \partial)$ is expected to categorify. One part of the conjecture states that the natural map

$$
K_{0}(R(\nu), \partial) \otimes_{\mathbb{O}_{p}} K_{0}\left(R\left(\nu^{\prime}\right), \partial\right) \longrightarrow K_{0}\left(R(\nu) \otimes_{\mathbb{k}} R\left(\nu^{\prime}\right), \partial\right)
$$

is an isomorphism for any weights $\nu, \nu^{\prime}$.

It is unclear how to extend the differentials to nonsimply-laced KLR algebras, since dots there have varying degrees depending on colors.

The Webster algebras [47] have a diagrammatic description analogous to that of the KLR algebras and their cyclotomic quotients. The diagrams are generated by red and 
black strands, such that red strands are labeled by positive weights and never cross each other, while black strands can carry dots and satisfy relations similar to nilHecke algebras. For instance, in the $A_{1}$ case, there are the following red-black crossings for the weight $k \in \mathbb{N}$ :
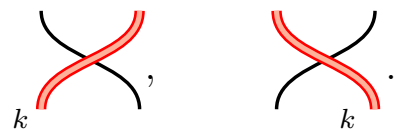

subject to some local relations, for instance:

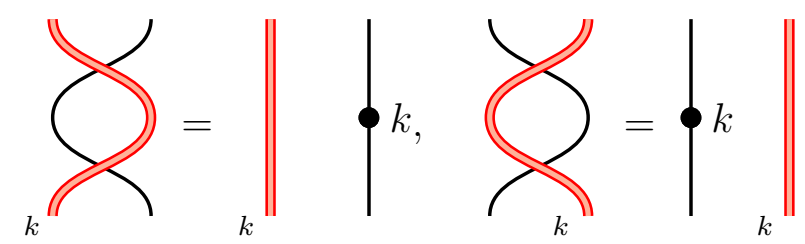

We refer the reader to $([47$, Section 2]) for the details.

One readily sees that, for any $\lambda \in \mathbb{F}_{p}$, the following differential on the new generators, together with the differential $\partial_{1}$ on the nilHecke algebra (equations (65), (66) ) defines a $p$-nilpotent local derivation on the Webster algebra.

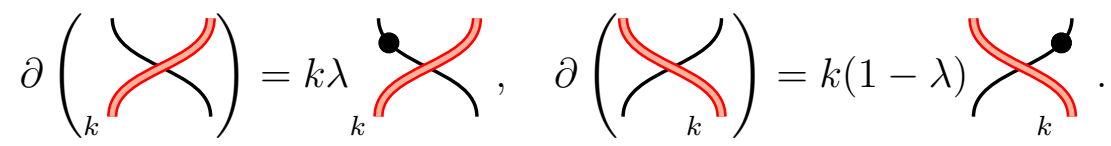

\section{References}

[1] M. Angel and R. Díaz, On N-differential graded algebras, J. Pure Appl. Algebra 210, 3, (2007), 673-683, math.DG/0504398.

[2] J. Bernstein and V. Luntz, Equivariant sheaves and functors, LNM 1578, (1994), Springer-Verlag.

[3] J. Bichon, N-complexes et algébres de Hopf, C. R. Acad. Sci. Paris., Ser. I 337 (2003), 441-444.

[4] C. Cibils, A. Solotar, and R. Wisbauer, N-complexes as functors, amplitude cohomology and fusion rules, Comm. Math. Phys. 272 (2007), 837-849, arXiv: math/0605569.

[5] L. Crane, I. Frenkel, Four-dimensional topological quantum field theory, Hopf categories, and the canonical bases, J. Math. Phys. 35, 5136-5154, (1994), arXiv: hep-th/9405183.

[6] M. Dubois-Violette, Generalized differential spaces with $d^{N}=0$ and the $q$ differential calculus, Czech. J. Phys. 46 (1996), 1227-1233, q-alg/9609012. 
[7] M. Dubois-Violette, $d^{N}=0$, q-alg/9710021.

[8] M. Dubois-Violette, Lectures on differentials, generalized differentials and on some examples related to theoretical physics, Contemp. Math. 294, AMS (2002), 59-94. math.QA/0005256.

[9] M. Dubois-Violette and R. Kerner, Universal q-differential calculus and q-analog of homological algebra, Acta Math. Univ. Comenianae vol. LXV 2 (1996), 175-188, q-alg/9608026.

[10] B. Elias, Y. Qi, An approach to categorification of some small quantum groups II, arXiv:1302.5478.

[11] B. Elias, Y. Qi, in preparation.

[12] S. I. Gelfand and Y. I. Manin, Methods of homological algebra (second edition), Springer, 2003.

[13] D. Happel, Triangulated categories in the representation theory of finite dimensional algebras, London Math. Soc. Lect. Note Ser. 119, (1988), Cambridge University Press.

[14] J. Jonsson, A refinement of amplitude homology and a generalization of discrete Morse theory, preprint, available at

http://www.math.kth.se/ jakobj/doc/submitted/amplitude.pdf.

[15] M. Kapranov, On the q-analogue of homological algebra, preprint, q-alg/9611005.

[16] C. Kassel and M. Wambst, Algébre homologique des N-complexes et homologie de Hochschild aux racines de l'unité, q-alg/9705001.

[17] B. Keller, Deriving DG categories, Ann. Scient Éc. Norm. Sup., 4e série, t. 27, 63-102, (1994).

[18] M. Khovanov, Hopfological algebra and categorification at a root of unity: the first steps, math.QA/0509083.

[19] M. Khovanov, How to categorify one-half of quantum $g l(1 \mid 2)$, arxiv:1007.3517.

[20] M. Khovanov and A. D. Lauda, A diagrammatic approach to categorification of quantum groups I, Represent. Theory 13 (2009), 309-347, arXiv:0803.4121.

[21] M. Khovanov, A. D. Lauda, A diagrammatic approach to categorification of quantum groups II, Tran. A.M.S. 363, (2009), 2685-2700, arXiv:0804.2080.

[22] M. Khovanov, A. D. Lauda, M. Mackaay, M. Stošić, Extended graphical calculus for categorified quantum sl(2), Mem. AMS 219, (2012), arXiv:1006.2866v1. 
[23] B. Kostant, S. Kumar, The nil Hecke ring and cohomology of $G$ / $P$ for a Kac-Moody group G. Adv. in Math. 62 (1986), 187-237.

[24] G. Kuperberg, Non-involutory Hopf algebras and 3-manifold invariants, Duke Math. J., 84 (1996), 83-129 arXiv:q-alg/9712047.

[25] A. Lauda, A categorification of quantum sl(2), Adv. Math. 225 (2010), 3327-3424, arXiv:0803.3652v3.

[26] R. Lipshitz, P. Ozsváth, D. Thurston, Bordered Heegaard Floer homology: invariance and pairing, arXiv:0810.0687.

[27] R. Lipshitz, P. Ozsvath, D. Thurston, Slicing planar grid diagrams: a gentle introduction to bordered Heegaard Floer homology, Proceedings of 17th Gökova Geometry-Topology Conference, (2010), 91-119, arXiv 0810.0695.

[28] G. Lusztig, Canonical bases arising from quantized enveloping algebras, J. Amer. Math. Soc. 3 no. 2, (1990), 447-498.

[29] G. Lusztig, Introduction to quantum groups, Progress in Mathematics, vol. 110. Birkhäuser, 1993.

[30] S. Majid, Some comments on bosonisation and biproducts, Czech. J. of Phy., 47, N. 2, 151-171, arXiv: q-alg/9512028.

[31] A. Malkin, Tensor product varieties and crystals: the ADE case. Duke Math. J. 116 no. 3 (2003), 477-524, arXiv: math.AG/0103025.

[32] L. Manivel, Symmetric functions, Schubert polynomials and degeneracy loci, SMF/AMS Texts and Monographs 6 (2001), AMS.

[33] W. Mayer, A new homology theory I, Ann. Math. 43 (1942), 370-380.

[34] W. Mayer, A new homology theory II, Ann. Math. 43 (1942), 594-605.

[35] S. Montgomery, Hopf algebras and their actions on rings, CBMS Regional conference series in mathematics 82, Amer. Math. Soc., 1993.

[36] H. Nakajima, Instantons on ALE spaces, quiver varieties, and Kac-Moody algebras, Duke Math. J. 76, no. 2, (1994) 365-416.

[37] H. Nakajima, Quiver varieties and tensor products, Invent. Math. 146 (2001), 399-449, arXiv: math/0103008.

[38] B. Pareigis, A non-commutative non-cocommutative Hopf algebra in "nature", Journal of Algebra 70, (1981), 356-374. 
[39] Y. Qi, Hopfological algebra, arXiv:1205.1814.

[40] N. Yu. Reshetikhin and V. G. Turaev, Ribbon graphs and their invariants derived from quantum groups, Comm. Math. Phys. 127, no. 1, (1990), 1-26.

[41] R. Rouquier, 2-Kac-Moody algebras, arXiv:0812.5023.

[42] S. Sarkaria, Combinatorial methods in topology, notes of Chandigarh Topology Seminar (1994-1995).

[43] S. Sarkaria, Some simplicial (co)homologies, IHES preprint 1995, available on http://kssarkaria.org/ .

[44] E. Spanier, The Mayer homology theory, Bull AMS 55 (1949), 102-112.

[45] M. Varagnolo and E. Vasserot, Canonical bases and KLR-algebras, J. Reine Angew. Math. 659, (2011), 67-100, arxiv:0901.3992.

[46] M. Wambst, Homologie cyclique et homologie simpliciale aux racines l'unité, $K$ theory 23, 4, (2001), 377-397.

[47] B. Webster, Knot invariants and higer representation theory I: diagrammatic and geometric categorification of tensor products, arXiv:1001.2020.

[48] B. Webster, Knot invariants and higer representation theory II: the categorification of quantum knot invariants, arXiv:1005.4559.

[49] H. Zheng, Categorification of integrable representations of quantum groups, arXiv:0803.3668.

Mikhail Khovanov, Department of Mathematics, Columbia University, New York, NY 10027 email: khovanov@math.columbia.edu

You Qi, Department of Mathematics, Columbia University, New York, NY 10027

email: yq2121@math.columbia.edu 